\title{
Experimental cytomegalovirus infections in a rat model : pathogenesis and treatment
}

Citation for published version (APA):

Kloover, J. S. (2002). Experimental cytomegalovirus infections in a rat model : pathogenesis and treatment. [Doctoral Thesis, Maastricht University]. Universiteit Maastricht. https://doi.org/10.26481/dis.20020614jk

Document status and date:

Published: 01/01/2002

DOI:

10.26481/dis.20020614jk

Document Version:

Publisher's PDF, also known as Version of record

\section{Please check the document version of this publication:}

- A submitted manuscript is the version of the article upon submission and before peer-review. There can be important differences between the submitted version and the official published version of record.

People interested in the research are advised to contact the author for the final version of the publication, or visit the DOI to the publisher's website.

- The final author version and the galley proof are versions of the publication after peer review.

- The final published version features the final layout of the paper including the volume, issue and page numbers.

Link to publication

\footnotetext{
General rights rights.

- You may freely distribute the URL identifying the publication in the public portal. please follow below link for the End User Agreement:

www.umlib.nl/taverne-license

Take down policy

If you believe that this document breaches copyright please contact us at:

repository@maastrichtuniversity.nl

providing details and we will investigate your claim.
}

Copyright and moral rights for the publications made accessible in the public portal are retained by the authors and/or other copyright owners and it is a condition of accessing publications that users recognise and abide by the legal requirements associated with these

- Users may download and print one copy of any publication from the public portal for the purpose of private study or research.

- You may not further distribute the material or use it for any profit-making activity or commercial gain

If the publication is distributed under the terms of Article $25 \mathrm{fa}$ of the Dutch Copyright Act, indicated by the "Taverne" license above, 


\section{Experimental Cytomegalovirus Infections in a Rat Model}

- Pathogenesis and Treatment -

Jeroen Steven Kloover 
ISBN nummer: 90-77017-90-9

Druk: Optima Grafische Communicatie, Rotterdam 


\title{
Experimental Cytomegalovirus Infections in a Rat Model
}

\author{
- Pathogenesis and Treatment -
}

\section{PROEFSCHRIFT}

ter verkrijging van de graad van doctor aan de Universiteit Maastricht, op gezag van de Rector Magnificus, Prof.Dr. A.C. Nieuwenhuijzen Kruseman, volgens het besluit van het College van Decanen, in het openbaar te verdedigen op vrijdag 14 juni 2002 om 12.00 uur

$$
\text { door }
$$

Jeroen Steven Kloover 


\section{Promotor}

Prof.dr. C.A. Bruggeman

Beoordelingscommissie

Prof.dr. M.J.A.P. Daemen (voorzitter)

Prof.dr. J.M.R. Balzarini (Katholieke Universiteit Leuven)

Prof.dr. J.P. van Hooff

Prof.dr. J.J. Manni

Prof.dr. P. Nieuwenhuis (Rijksuniversiteit Groningen) 
Aan

Susan

Papa en Mama Annemieke

Ter herinnering aan mijn grootouders 


\section{Contents}

List of abbreviations

General introduction and aims of the study

Chapter 1 Review of the literature

Chapter 2 Rat cytomegalovirus replication in the salivary glands is exclusively confined to striated duct cells.

Chapter 3 Persistent rat cytomegalovirus (RCMV) infection of the salivary glands contributes to the anti-RCMV antibody response.

Chapter 4 A rat cytomegalovirus strain with a disruption of the $\mathrm{r} 144 \mathrm{MHC}$ class I-like gene is attenuated in the acute phase of infection in neonatal rats.

Chapter 5 Rat cytomegalovirus infection in kidney allograft recipients is associated with increased expression of Intracellular Adhesion Molecule-1 Vascular Adhesion Molecule-1 and their ligands Leukocyte Function Antigen-1 and Very Late Antigen-4 in the graft.

Chapter 6 Effective treatment of experimental cytomegalovirus-induced encephalo-meningitis in immunocompromised rats with HPMPC.

Chapter 7 Effect of desferioxamine (DFO) and calcium trinatrium diethylenetriaminepentaacetic acid (DTPA) on rat cytomegalovirus replication in vitro and in vivo.

Chapter 8 General discussion and summary

Samenvatting

List of publications

Dankwoord / acknowledgements

Curriculum vitae

References 


\section{List of abbreviations}

$\begin{array}{ll}\text { AIDS } & \text { acquired immune deficiency syndrome } \\ \text { BMT } & \text { bone marrow transplantation } \\ \text { CADI } & \text { chronic allograft damage index } \\ \text { CMV } & \text { cytomegalovirus } \\ \text { CNS } & \text { central nervous system } \\ \text { CTL } & \text { cytotoxic T lymphocytes } \\ \text { DFO } & \text { desferioxamine } \\ \text { DHPG } & \text { 9-(1,3-dihydroxy-2-propoxymethyl)guanine } \\ \text { DTPA } & \text { calcium trinatrium diethylenetriaminepentaacetic acid } \\ \text { E } & \text { early } \\ \text { EBV } & \text { Epstein Barr virus } \\ \text { ER } & \text { endoplasmic reticulum } \\ \text { gp } & \text { glycoprotein } \\ \text { GvHD } & \text { graft versus host disease } \\ \text { HAART } & \text { highly active anti-retroviral therapy } \\ \text { HHV } & \text { human herpes virus } \\ \text { HIS } & \text { hyper immune serum } \\ \text { HIV } & \text { human immune deficiency virus } \\ \text { HLA } & \text { human leukocyte antigen }\end{array}$

HPMPC (s)-1-(3-hydroxy-2-phosphononylmethoxypropyl)cytosine HSV herpes simplex virus

IE immediate early

ICAM-1 intracellular adhesion molecule-1

IL-1 interleukin-1

IL-2-R interleukin-2 receptor

IFN-gamma Interferon gamma

IRV immune recovery vitritis

iv intravenous

L late

LFA-1 leukocyte function antigen-1

MCMV murine cytomegalovirus

MHC major histocompatibility complex

mRNA messenger RNA

NK natural killer

PBSCT peripheral blood stem cell transplantation

RCMV rat cytomegalovirus 
TAA transplant associated arteriosclerosis

TNF-alpha tumor necrosis factor-alpha

US unique short

UL unique long

VCAM-1 vascular adhesion molecule-1

VLA-4 very late antigen-4

VZV varicella zoster virus

WT wild type 


\section{General introduction and aims of the study}

Cytomegalovirus (CMV) is an important human pathogen due to the high seroprevalence of the virus in the normal population and the severity of CMV disease in the immunocompromised patient, like in transplant recipients and AIDS patients. The best hypothetical therapy for CMV infections in the immunocompromised patient is restoration of immune effector functions. For example, a drastic decrease in the incidence of CMV disease in AIDS patients could be appreciated since the introduction of HAART. Reconstruction of immune function can be realized in a subgroup of AIDS patients, but it is unfeasible in transplant recipients, since inadequate immunosuppression is a risk factor for allograft rejection. Current treatment modalities for CMV disease include potent inhibitors of $\mathrm{CMV}$ replication, such as ganciclovir, cidofovir and foscarnet. However, the treatment of severe CMV disease remains difficult due to the limited specificity and serious side effects of the compounds and deterioration of the patient is often seen, despite the administration of high dose antiviral agents. Therefore, the search for new treatment modalities capable of reducing viral replication and/or recurrence and subsequently CMV disease is of great importance. Animal models have been shown to be extremely useful for studying viral pathogenesis and the development of new antiviral treatments. The rat model, using rat CMV (RCMV), has been used extensively in various fields of research and is now widely accepted as a good model for CMV infections in humans. The aim of this thesis was to study some aspects of CMV pathogenesis and treatment of CMV infection in various experimental settings, using the RCMV/rat model.

The outline of the study is as follows. Chapter $\mathbf{2}$ and $\mathbf{3}$ focus on RCMV replication in the salivary gland, the primary organ for RCMV replication during chronic infection and the biological significance of RCMV replication in salivary gland cells on the systemic infection was studied. Chapter 4 describes infection of neonatal animals with RCMV of which the MHC class I homolog ( $r 144)$ has been disrupted (RCMV $\Delta \mathrm{r} 144)$. In contrast to adult rats, in which immunosuppression is mandatory for disseminated infection, the use of neonatal rats enabled us to study the interaction of the $\mathrm{r} 144$ gene product with an unaffected, but immature natural immune system. Chapter $\mathbf{5}$ is devoted to RCMV-mediated enhancement of chronic renal allograft rejection. In this chapter, the additive effect of RCMV infection on immune activation was subject of study. Treatment of CMV disease is described in chapter 6 and 7. In chapter 6, direct inhibition of viral replication in brains of immunocompromised rats was studied, whereas in chapter 7 , two drugs with putative antiviral activity and supposed ability to modulate RCMV-induced immunopathogenesis were studied. In chapter $\mathbf{8}$, the results and conclusions of this thesis are summarized and discussed. 
Chapter 1

\section{Review of the literature}




\section{Viral Structure and characteristics}

Cytomegalovirus (CMV) is a member of the herpesviridae. Membership of the herpesviridae is based on the composition of the virion: a core containing linear doublestranded DNA, an icosadeltahedral capsid (diameter 100-110 nm) containing 162 capsomeres, presence of an amorphous material that surrounds the capsid, called the tegument and an envelope containing viral glycoprotein spikes (434). Cytomegaloviruses are classified in a subfamily of the beta-herpesviridae, due to the restricted host range, long reproduction cycle, the characteristic cellular morphological alteration during viral replication (cytomegaly, owls eye appearance) and the ability to persist in the host in a latent form.

The first step in CMV infection, is attachment of the virion to the membrane of the cell. $\mathrm{CMV}$ enters fibroblasts by a process involving $\mathrm{pH}$-dependent fusion (112). The initial event in this process is attachment to extracellular heparan sulfate (113). This binding is followed by attachment of CMV envelope glycoproteins (gp), such as $\mathrm{gpB}$ and $\mathrm{gpH}$ with cell membrane components. Other host membrane molecules, such as CD13 (476), a $92.5 \mathrm{kDa}$ phosphoprotein (260) and annexin II (554) have been suggested to be involved in CMV attachment to the target cell.

The mechanism of cell penetration by CMV appears to differ between cell types. In contrast to fibroblasts, cell entrance into endothelial and astrocytoma cells appears to be mediated by endocytosis, rather than fusion with the cytoplasmic membrane (56). After penetration of the cell membrane, the virus is uncoated and the viral genome is transported to the nucleus. Expression of the CMV genome is controlled by a cascade synthesis of proteins (197). The first proteins to be synthesized are immediate early (IE) proteins. These proteins have a regulatory function and permit transcription of early $(E)$ messenger RNA (mRNA). During the E phase of replication, DNA replication occurs. The transcription of late $(\mathrm{L})$ mRNA takes place after completion of DNA replication and $\mathrm{L}$ mRNA encodes structural proteins, such as viral capsides (UL86), tegument protein (UL83) and envelope glycoproteins.

During virus assembly, the CMV DNA is packaged in the nucleus into preformed capsids and a viral envelope and the viral matrix (tegument) is acquired at the nuclear cell membrane. However, it is thought that the final viral envelopement occurs at the cellular

membrane $(185,359)$. Transport to the cellular membrane may be mediated by cytoplasmic vesicles derived from the Golgi apparatus (359). The characterized owls eye appearance is due to an excessively expanded Golgi apparatus. 


\section{Epidemiology of human cytomegalovirus infections}

Presence of $\mathrm{CMV}$ in the normal population is common and seroprevalence in healthy individuals can be as high as $80-90 \%(183,221,368,377)$. However, these data probably even underestimate the real prevalence of CMV since $5-20 \%$ of serologically negative individuals will harbour latent viral DNA $(48,291,470,501)$. Seroprevalence is directly correlated with socio-economic status $(280,328)$ and age $(86,192,368)$. Most individuals become infected during childhood $(255,460)$ or adolesence $(18,436)$.

Congenital CMV infection is unquestionably the most common intra-uterine infection in humans and has been reported to vary between $0.2 \%-6.8 \%$ of all new-borns $(8,128,129,204$, 447). After birth and during the first months of life, children are most frequently infected by vertical transmission of the virus (contaminated genital secretions and/or breast milk) (221. 368). Thereafter a significant number of children is infected at day care centers in which close contact and thus (horizontal) viral transmission, is inevitable $(5,6,369,384)$. In addition, infected children can be a source for viral transmission to their parents (386) or day care employees $(4,6,363,385)$. However, in a recent report by Murph et al., day care workers were not found to be at risk for acquiring CMV (364). This is possibly due to an increased awareness about (personal) hygiene in these institutions.

Another period during which CMV infection is common, is during adolescence when infection has been associated with heterosexual $(168,364,479,518)$ and homosexual $(109,136)$ activity.

\section{Cytomegalovirus infections in the immunocompetent host}

$\mathrm{CMV}$ infection of the immunocompetent host is usually asymptomatic or may result in a mononucleosis like syndrome in approximately $10 \%$ of infected individuals. The dominant symptoms are fever, malaise, myalgia, atypical lymphocytosis and abnormal liver functions (251, 265). However, also splenomegaly and lymphadenopathy have been reported. The syndrome is further characterised by a persistent viruria in almost all patients $(251,265)$.

Although few data are available concerning the incidence of CMV mononucleosis, it is thought that the condition is relatively common and runs a mild course. However, few cases progress into life threatening CMV disease (141). In these patients, the most commonly infected organs are liver, CNS and lungs. Patients with severe CMV disease respond well to ganciclovir or foscarnet treatment $(141,337)$.

The host immune response to CMV infections can be divided in non-specific and specific immune surveillance. The humoral immune response does not appear to play a significant role in controlling acute CMV infections, since viral infection or recurrence is rapidly cleared by the cellular immune system. However, anti-CMV immunoglobulins are able to limit recurrent CMV disease effectively $(11,12,395)$. Cytotoxic CD8+ T lymphocytes are important for controlling CMV infections $(359,426)$. The number of CD8+ cells in blood 
correlates with the severity of CMV disease (308). The importance of these cells in controlling CMV disease is further shown by results of adoptive transfer studies in posttransplant disease $(431,432)$. The biological significance of CD4+ cells is demonstrated by the rapid increase in incidence of CMV disease in AIDS patients with a progressive decline in CD4 counts $(117,240)$. Besides the specific cellular immune response, natural killer (NK) cells, which are part of the non-specific cellular immune system, were also shown to be important for CMV immune surveillance (52).

The physiological significance of the humoral $(154,459)$ and cellular, i.e. CD4+ T cells (248, 249), CD8+ T cells $(278,420,421)$ and NK cells $(80,544)$, components of the immune system on controlling $\mathrm{CMV}$ infection was corroborated extensively in the murine/MCMV model.

\section{Cytomegalovirus and latency}

The ability to enter a latent state, i.e. absence of infectious virus as measured by plaque assay but persistence of viral DNA, is a characteristic shared by all herpesviruses (435). CMV has been known to be capable of infecting a wide range of organs in immunosuppressed patients, resulting in multiple potential sites for CMV latency (212). In humans, emphasis has been laid on peripheral mononuclear cells $(64,454,466,514)$ and their progenitors $(203,271,272,346,357,461)$ as sites for CMV latency. The number of viral genomes per latently infected hematopoietic cell of healthy blood donors is very low with only 2-13 viral genome copies per cell (466). However, CMV replication in mononuclear cells may be abortive or restricted to early events of gene expression (231, 429, 515). Interestingly, differentiation of mononuclear cells makes them fully permissive for $\operatorname{CMV}(203,231,477)$.

Recent data suggest that CMV reactivation and replication in myeloid cells largely depends on immune activation. Cytokines, i.e. tumor necrosis factor-alpha (TNF-alpha) and interferon-gamma (IFN-gamma) and the presence of CD8+ T cells enhances CMV replication in monocyte-derived macrophages (477). In addition, TNF-alpha is able to enhance the immediate early (IE) promotor of CMV in myeloid cells $(412,504)$. Furthermore, reactivation of latent $\mathrm{CMV}$ from latently infected peripheral blood mononuclear cells was established by allogeneic stimulation of these cells in vitro (478).

Another mechanism for viral reactivation has been described, which acts independently of inflammatory mediators (411). Prösch et al. described that cathecholamines, measured in patients with an acute myocardial infarction, stimulates the major IE enhancer-promotor of CMV, similar to TNF-alpha, although via different cellular pathways (413).

In the murine model using murine CMV (MCMV), latent MCMV has been detected in blood leukocytes, endothelial cells, bone marrow, salivary gland, heart, liver, brain, kidney, adrenal glands and spleen $(111,269,270,358)$. However, the lungs were shown to be the major organ site for viral latency (31, 283). Reactivation of MCMV could be established by tissue explant cultures $(347,406)$ or, in vivo, after immunosuppression of latently infected mice (31. 
284, 419, 503). It was shown in this model that 1 ) reactivation does not accumulate over time after initiation, 2) reactivation is distributed in the murine lung in a patchwork pattern and 3) that reactivation of latent virus is a multistep process ${ }^{(285)}$. Latency-associated transcripts were found in the murine model $(200,284)$ and in humans $(273,289,322)$. These findings suggest that induction of CMV reactivation is a tightly regulated, non-stochastic process.

\section{Cytomegalovirus and immune evasion}

During a period of thousands of years, the intimate relation of herpesviruses with their host, has resulted in multiple mechanisms to elude to host immune system (340). An effective mechanism to escape from immune surveillance is to limit the number of antigens expressed on the cellular surface. The immune system cannot kill an infected cell when it is not able to identify this cell as being infected. Therefore, the ultimate form of immune evasion is latency. The key characteristic of latency is the persistence of viral DNA without production of infectious virus with minimal cellular expression of viral antigens (48, 291, 470, 501). However, it should be noted that latency-associated transcripts of CMV may elicit a humoral immune response $(273,289,322)$.

Another mechanism for avoiding detection by the immune system, is by preventing major histocompatibility complex (MHC) class I-mediated presentation of antigens to T lymphocytes. The gene product of UL 83, pp65, is able to inhibit immediate-early peptide presentation (186). In addition, CMV infection is associated with a down-regulation of two cellular aminopeptidases (401), i.e. CD10 and CD13, which are responsible for trimming peptides during their insertion into MHC class I and II molecules $(290,362)$.

However, the most effective way of inhibiting MHC class I recognition, is interference with the MHC class I assembly itself. It has been known that human CMV (CMV) downregulates cellular human leukocyte antigen (HLA) class I molecules in order to protect infected cells from MHC class I restricted cytotoxic T Lymphocytes mediated lysis (40). Gene products of US2 $(246)$, US3 $(9,247)$, US6 $(10,213)$ and US11 $(545)$ have been shown to interfere with the cellular expression of MHC class I. Genes with a similar function were found on the genome of MCMV (m152 (565), m06 (424)) and m04 (264)).

According to the missing self hypothesis, absence of HLA / MHC class I molecules on the cellular membrane renders a cell susceptible for NK cell lysis ${ }^{(316)}$. For optimal evasion of immune surveillance the virus must encode factors which are able to elude the innate immune response. It was hypothesized that a virally encoded $\mathrm{MHC}$ class I homolog could function as a decoy for NK cell surveillance.

CMV encodes an HLA class I homolog, named UL18 (39). Similar genes were found in the genome of murine $\left(\mathrm{m} 144,{ }^{(418)}\right)$ and rat $\left(\mathrm{r} 144,{ }^{(531)}\right)$ CMV. CMV encoded HLA class I homolog associates with beta-2-microglobulin (72), is able to bind peptides (151) and was found in peripheral blood mononuclear cells of organ transplant recipients (207). In contrast 
to CMV, MCMV derived "class I" molecules are not able to bind endogenous peptides, which may be mediated by a deletion within the m144 alpha- 2 domain encoding the predicted peptide-binding groove (92).

UL18 expression on target cells has been shown to interact with the CD94/NKG2 receptor (418) and the leukocyte immunoglobulin-like receptor-1 (LIR-1) receptor (114). CD94 is a member of the C-type lectin superfamily and associates with NKG2 family of C-type lectins $\left({ }^{87,295)}\right.$. CD94/NKG2 heterodimers are expressed on NK cells and have been shown to recognize MHC class I and to inhibit NK cell function. Another viral protein, gpUL40, which contains an HLA-E ligand, also interacts with the CD94/NKG2 complex and is able to inhibit NK cell surveillance $(517,523)$. LIR-1 is a member of the $\mathrm{Ig}$ superfamily and is predominantly expressed on B cells and monocytes (114).

There is strong evidence from the murine model that expression of the MCMV-encoded MHC class I molecule on the cellular surface protects the infected cell from NK cell lysis in vivo ${ }^{(155)}$ as well as in vitro ${ }^{(282)}$. It should be noted that in vitro only a partial inhibition of NK cell cytotoxicity was observed (282).

Furthermore, it was shown that $\mathrm{m} 144$ protected lymphoma cells transfected with $\mathrm{m} 144$ from NK cell mediated rejection in vivo (116). Interestingly, expression of m144 reduced the recruitment of leukocytes and NK cells and the level NK cell activation (116). This suggests that the MHC class I homolog may influence the local interaction between cells of the innate immune system, such as antigen presenting cells (APC) and NK cells. Especially, since it is known that monocyte-derived cytokines are important for NK cell activation and infiltration $(443,473)$. Leong et al. proposed a similar role for UL18 $(306)$, based on the interaction of this molecule with LIR-1, which is predominantly expressed on monocytes and B lymphocytes (114).

Recent studies have shown that also CD4+ T cells are important in controlling CMV infections (122, 249, 321). There is evidence that besides interference with HLA/MHC class I complexes, CMV has also been shown to inhibit HLA class II presentation by inhibition of IFN-gamma (354).

CMV encodes 4 genes which show homology to chemokine receptors, namely UL33,

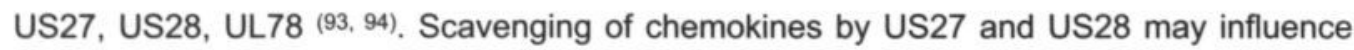
infiltration of leukocytes (26). The ligands and functions of UL33 and UL78 are still unknown. However, deletion mutant viruses (MCMV-d-m33, RCMV-d-r33, RCMV-d-r78) have been generated for the rat $(41,44)$ and murine system (124). Disruption of the $r 33$ and m33 gene was associated with an absence of viral replication in salivary glands, whereas deletion of the $r 78$ resulted in a lower replication efficiency than wild type (WT) virus and infected cells developed a syncytium-like appearance in vitro. Furthermore a significantly higher survival was seen in RCMV-d-r78 infected rats than in WT RCMV infected animals (41). 
Although our insights in immune evasive strategies by CMV are accumulating, further research is required, since full knowledge about these strategies will result in novel diagnostic and therapeutic modalities.

\section{Clinical aspects of cytomegalovirus infection}

Congenital cytomegalovirus infection

$\mathrm{CMV}$ is the most common congenital infection in humans and the prevalence of congenital CMV ranges from $0.2 \%$ to $6.8 \%$ of all new-borns $(8,32,89,128,393,447)$. Of all infected newborns, $5 \%$ are born with CMV disease, which is characterized by splenomegaly, hepatomegaly, prolonged neonatal jaundice, thrombocytopenic purpura and/or pneumonitis $(128,393)$. Other consequences of intra-uterine CMV infection are fetal growth retardation, preterm birth and inta-uterine death. Prognosis in this group is poor and during follow-up, children mainly suffer from neurological deficits, such as microcephaly, cerebral palsy, epilepsy, mental retardation, chorioretinitis, optic atrophy, delayed psychomotor development and especially sensorineural hearing loss $(65,106,128,387$. 393).

The majority of the asymptomatic CMV infected newborns will develop normally. However, $5 \%$ will suffer from long term sequelae of which sensorineural hearing loss is the most common $(166,167,393)$. Sensorineural hearing loss is fluctuating, albeit progressive and is in $50 \%$ bilateral (167). Of concern is the fact that population wide screening of acoustic function in asymptomatic CMV infected neonates fails in over $50 \%$ (166). A careful follow-up of these patients is thus warranted.

The factors that influence the occurrence of congenital CMV infection and those that influence the occurrence of primary CMV infection in pregnant women include race, sexual activity, young age, unmarried status and lower socio-economic status (168, 364, 479, 495, 518). CMV infection during pregnancy is either from reactivation of latent virus, infection with a different strain or a primary CMV infection. One in every 100 women will encounter a primary CMV challenge during pregnancy and approximately $40 \%$ will transmit the infection to their foetus (393). Newborns with CMV disease as a result from maternal reactivation or re-infection with a different strain have a better prognosis that new-borns from mothers with a primary infection $(128,169,496)$.

A reliable diagnostic tool for predicting which newborn will develop CMV disease is still missing. Serological status of the mother is of little value for predicting vertical transmission of virus, since not all seropositive women will transmit the virus to their child in utero. However, negative serostatus will define a subgroup of women who are at low risk for viral transmission to their fetus.

Culture of the virus (296) is considered diagnostic for CMV infection of the fetus, since 
amniotic fluid is mostly produced by the fetus and culture of which thus closely represents fetal CMV status. Also detection of viral DNA in amniotic fluid by PCR (245) has been used for diagnosing $\mathrm{CMV}$ infected fetuses. However, cases are described in which children with CMV DNA containing amniotic fluid remained unaffected (286, 297, 312, 441). Those cases are associated with a low number of viral DNA copies. Although the results from amniotic fluid sampling should be handled with care, this technique will be one of the most important diagnostic tools for congenital CMV disease in utero $(286,297,312,441)$ since the amount of viral DNA present may be an indicator for the severity of CMV disease (297). However, the true value of the PCR technology for the diagnosis of congenital CMV disease will have to be established.

\section{Cytomegalovirus infection in the HIV infected patient}

Since its discovery in the early 1980's $(33,175,243)$, human immune deficiency virus (HIV) infection is a major cause of morbidity and mortality on a world-wide basis. HIV selectively infects peripheral $C D 4+T$ lymphocytes and interferes with normal $T$ cell function, resulting in a depletion of CD4+ lymphocytes (156). Below the threshold of 100-400 CD4+ T cells/ul blood, patients are prone to be infected with opportunistic pathogens, such as CMV and are likely to develop opportunistic malignancies (115).

CMV end-organ disease occurs approximately in $21-44 \%$ of patients suffering from AIDS and is primarily associated with low CD4+ counts ( $<50$ cells $/ \mu l)$ (237). Retinitis accounts for over $85 \%$ of active CMV disease, followed by gastro-intestinal (16\%) and central nervous system disease $(1 \%)(174,240,400)$.

CMV retinitis may be the initial manifestation of AIDS. Onset of CMV retinitis may be unilateral or bilateral and usually starts with a focal discrete lesion, which eventually will progress in a diffuse retinal involvement (214). The initial lesions are characterized by retinal edema, yellow-white granular dots and vascular cuffing (due to perivascular mononuclear cell infiltrates) and attenuation. The clinical presentation depends on the ocular structures involved (214). Lesion of the peripheral retina is usually found at indirect fundoscopy in the asymptomatic patient. Vitreous involvement results in a blurred vision and floaters (239). Infection of the macula leads to a drastic decrease in visual acuity, metamorphopsia and micropsia. The end result of CMV retinitis is a thin atrophic and/or necrotic retina, prone to retinal detachment $(70,170)$.

Gastro-intestinal involvement encompasses infection of the oral cavity, esophagus / stomach (244,400) and the intestines (132, 258, 400). Neurological manifestations include ventriculo-encephalitis $(223,442)$, polyradiculopathy $(16,108)$, spinal cord and peripheral nerve involvement $(16,191)$ and may complicate AIDS dementia complex (161). In addition, also CMV induced adrenalitis ${ }^{(414)}$ and pneumonitis (543) have been described.

CMV can be considered exclusively as an opportunistic pathogen but its relation with 
HIV, however, is more complex. It is known that CMV can augment HIV infection in vitro $(34,35,123,361)$ as well as in vivo ${ }^{(36,159)}$. Additionally, co-infection of HIV with CMV will result in an increased lysis of cells (88). This indicates that CMV might have an additional effect to the HIV-mediated rapid decrease in CD4+ count.

Since the introduction of therapeutic regimens that include protease inhibitors, termed: highly active anti-retroviral therapy (HAART), a striking reduction of CMV related morbidity in AIDS patients has been seen, which is associated with an increase in CD4 cell count (225, 239, 301, 383, 457). Conversely, failure of HAART (i.e. decrease in CD4+ count) is associated with CMV relapses (482). Cessation of CMV prophylaxis is possible in AIDS patients with HAART related immuno-reconstruction $(352,522,538)$. Viral load and CD4+ count should be monitored thoroughly since relapses have been described within 4 months after initiation of HAART with adequate suppression of HIV (241).

On the other hand, morbidity that does occur, is no less serious since the introduction of HAART. Immune-mediated tissue damage at the site of infection (225) may occur due to the HAART induced improved immune function $(22,239,383)$. For example, CMV retinitis before initiation of HAART was characterized by minimal intra-ocular inflammation whereas in the post-HAART era, inflammation is more prominent and may result in tissue destruction. This immune recovery vitritis (IRV) is characterized by posterior segment inflammation, including vitritis, papillitis and macular changes and an absence of active CMV retinitis (256. 257).

\section{Cytomegalovirus and vascular pathology}

CMV has been associated with the process of atherosclerosis, restenosis, transplant associated atherosclerosis (TAA) and inflammatory aortic disease. These conditions encompass different clinical and histopathological entities. For example, atherosclerosis develops over years, whereas restenosis after angioplasty usually progresses in a period of weeks or months. TAA differs from classical atherosclerosis by a generalized, concentric involvement of the vascular structures in the allografted organ. Although there are a lot of differences between these conditions, some pathophysiological mechanisms are similar.

Atherosclerosis is a chronic inflammatory disease of the large and medium sized arteries ${ }^{437,438)}$. It is now generally accepted that atherosclerosis develops as a response to injury (439). The first and perhaps the most important step is dysfunction of the endothelial cells. The first histological characteristic of atherosclerosis is the presence of the fatty streak, an inflammatory lesion consisting of monocytes derived macrophages and T lymphocytes $(437,438$ ). In persons with hypercholesterolaemia, the influx of these cells is preceded by extracellular deposition of lipids $(437,438)$. These fatty streaks are already detectable in coronary arteries and aortas of children before the age of $10(341,502)$. Fatty 
streaks will progress into atherosclerotic plaques $(149,150,335,336)$ and progression is characterized by a continued inflammation, smooth muscle cell proliferation and formation of fibrous tissue. Eventually, a fibrous cap is formed covering a core of lipid and necrotic material. Rupture of the plaque or thrombolisation may lead to acute vascular accidents (437, 438). The etiology of atherosclerosis is multi-factorial and include high levels of serum cholesterol, smoking, diabetes mellitus, hypertension, genetic predisposition and infectious micro-organisms, especially herpesviruses and Chlamydia pneumoniae (219, 311). Multiple micro-organisms may be present in the artherosclorotic lesion (96) and it was recently shown that the risk of coronary artery disease is related to the total pathogen burden through inflammatory responses ${ }^{(564)}$.

CMV has been linked to atherosclerotic disease based on serological data $(3,484,485,532$, 563), although not all authors found this association $(7,139,433)$. It has been shown that CMV is able to elicit a subclinical inflammatory response, as measured by C-reactive protein, in a subgroup of patients and that these particular patients are at a higher risk for developing atherosclerosis than infected patients without the subclinical inflammatory response (563).

CMV-DNA has been detected in the atherosclerotic vascular wall $(96,211,344,345)$. Indeed, vascular structures have been suggested to be a potential site for latency $(211,344)$ and/or persistence (163). Data from in vitro studies showed that a wide variety of cell types are permissive for CMV, such as monocytes/macrophages, fibroblasts, smooth muscle cells and endothelial cells. However, in contrast to viral replication in fibroblasts, permissiveness of endothelial cells for CMV is restricted (536) and depends on the activation state of the cell $(465,537)$ and on the virus strain used $(182,323,464)$. From studies in animal models it is known that CMV increases the adhesion of leukocytes to and augments lipid accumulation in endothelial cells (487). Also, virus-induced morphological alteration of endothelial cells and subendothelial space of the large vessels could be detected by electron microscopy $445,120$. 486). These data indicate that CMV mediated endothelial cell dysfunction contributes significantly to the inflammation-mediated progression of atheroscerosis. Another important event in the development of atherosclerosis is the proliferation and migration of smooth muscle cells (SMC). This process may be mediated by the production of growth factors and cytokines from macrophages and/or endothelial cells in response to vessel injury. Of special interest is the fact that the US28 gene product, a chemokine receptor encoded by CMV, is able to induce SMC migration (509).

CMV also enhances the development of restenosis after atherectomy or balloon angioplasty $(490,559)$. It was shown that CMV was able to inhibit the transactivational activity of the p53 suppressor gene by the interaction of p53 with IE2-84, an immediate early gene product of CMV. Inhibition of p53 abolishes p53-mediated cell cycle arrest of SMC and the interaction between p53 and IE2-84 inhibits CMV induced apoptosis (562). Both factors contribute to excessive SMC proliferation, which in turn leads to lumen narrowing. Besides 
SMC proliferation, CMV-induced immune activation after balloon injury also contributes to the process of restenosis in a similar manner as was described for the process of atheroscerosis. Animal experiments, using rodents subjected to balloon denudation and infected with the appropriate virus, support these findings in humans $(397,398,560,561)$.

TAA, i.e. chronic rejection, is associated with an acute onset, generalized and concentric involvement of the entire vessel, persistent perivascular inflammation and an intact internal elastic lamina $(50,51,530)$. Cytomegalovirus has been suggested to be associated with TAA, leading to an increased rate of graft rejection, more severe graft atherosclerosis and lower survival rates $(138,148,193,277,319,339)$. However, some groups failed to find a positive correlation between CMV infection and TAA (445). A positive correlation between TAA and CMV infection was found in cardiac allografted rats (302) and mice ${ }^{85}$. Also, in rats receiving an aorta allograft, it was demonstrated that CMV infection enhanced the generation of a prominent inflammatory response with presence of inflammatory cells and SMC proliferation in the allograft, resulting in neointima formation (304). The RCMV induced enhancement of TAA could be diminished by administration of triple therapy consisting of cyclosporin, methylprednisolone and azathioprine (305) or antiviral therapy (303). This indicates that viral replication,as well as the immune response following transplantation are important for the development of TAA.

Atherosclerosis is a multifactorial disease, although inflammation plays a key role in this process. Several reports have suggested a relationship between CMV infection and enhancement of vascular pathology. However, further research on how CMV enhances these vascular changes is warranted.

\section{Cytomegalovirus and hemapoietic stem cell transplantation}

Bone marrow transplantation (BMT) is a widely accepted component of the chemotherapeutic regimen for hematologic malignancies, bone marrow failure and congenital disorders of the lymphohemopoietic system (19). Hematopoietic stem cells can be derived from bone marrow, peripheral blood, cord blood and fetal liver (at 10-14 weeks of gestation) of a donor. In the absence of a syngeneic donor, an HLA matched sibling donor is the best choice. However, marrow derived from non-related HLA-matched donor is also widely used. The incidence of graft rejection and graft-versus-host disease (GvHD) is directly proportional to the level of mismatch $(121,325,492)$.

CMV disease is a major cause of morbidity and mortality in both allogeneic BMT (68, 348) and peripheral blood stem cell transplantation (PBSCT) ${ }^{(224)}$. During the 1980s, reactivation of CMV infection in CMV seropositive BMT recipients has been estimated to occur up to $65 \%$ of those patients $(355,548$ ). The incidence of CMV disease was reported $20-35 \%$ in seropositive bone marrow allografted patients $(348,350)$. Pneumonia is the most frequent manifestation with a high mortality rate up to $80 \%(145,348,425)$. The clinical presentation 
includes fever, nonproductive cough, tachypnea and hypoxemia $(46,366)$. Besides the lungs, also the gastro-intestinal tract and retina can be involved, although less frequently (507). Previous to the introduction of CMV prophylaxis, CMV disease occurred 1-4 months after transplantation (350). However, with current potent anti-CMV prophylaxis, the peak incidence of CMV disease occurs not until 100 days after transplantation $(63,279)$.

CMV seropositive bone marrow recipients are at the highest risk for CMV disease, regardless of the serological status of the donor (187, 229, 349, 355). Seronegative patients receiving marrow from seropositive donors are at a lower risk for CMV associated disease than their CMV seropositive counterparts with either bone marrow from seropositive- or seronegative donors. The importance of allogeneic stimulation of latent virus in the recipient for developing CMV disease was shown by Soderberg-Naucler (478). The fact that the serostatus of the donor is of little importance is surprising, since it is thought that bonemarrow progenitors may serve as a reservoir for $\operatorname{CMV}(203,271,272,346,357,461)$. Seronegative patients receiving haemopoetic stem cells from seronegative donors are at the lowest risk for CMV infection (317). Other risk factors for developing CMV disease include high viral load (61,350), GvHD $(229,550)$, transplants from volunteer unrelated donor $(327,512)$ or mismatched related donors (317) and a delayed reconstitution of the CMV specific cytotoxic T cell response $(308,417)$.

The CTL response is important in controlling CMV infections. For example, patients receiving T-cell depleted grafts have an increased risk for developing CMV infections (218, 327). Also, reconstitution of CMV specific CD8+ lymphocytes has been shown to be protective in BMT recipients $(431,432,467)$. Also viral factors may influence onset and/or severity of CMV disease. There is increasing evidence that strain differences based on the CMV glycoprotein B may contribute to the virulence of CMV in BMT recipients (171, 520).

\section{Cytomegalovirus infection and solid organ transplantations}

Solid organ transplantations, such as liver, heart and lung transplantations, are currently available for treatment of various organ end-stage diseases in humans. Outcome is related to the surgical procedure, immunosuppression, allograft rejection and infections (253). The post transplant period can be divided into three phases, depending on the type of microbiological challenge ${ }^{(389)}$. During the first month, infectious complications are related to surgical complications. The microbiological challenge in solid organ transplant recipients during this period is similar to the microbiological threat after general surgery. The second period, i.e. the period between the second and sixth month post transplantation, opportunistic infections, such as CMV, occur. From 6 months after transplantation onward, patients encounter the 'normal' microbiological challenge as seen in the general community.

CMV infections are common in solid organ transplant recipients (137). CMV infection may 
be the result from viral transmission via an infected organ or blood products (primary infection or super-infection) or may be due to reactivation of latent virus already present in the recipient (secondary infection). Symptomatic CMV disease occurs in $8 \%$ of kidney-, $29 \%$ of liver-, $25 \%$ of heart-, $50 \%$ of pancreas- (or kidney/pancreas), $22 \%$ of small boweland $39 \%$ of heart/lung transplant recipients ${ }^{(389)}$.

Risk factors for CMV disease include transplanting an organ from a CMV-seropositive individual to a CMV -seronegative recipient, the level of immunosuppression (especially with antilymphocyte preparations, such as OKT3 monoclonal antibodies) and clinical condition of the patient (529). CMV disease can involve lung, liver, gastro-intestinal, renal and/or retinal disease.

CMV infection has been associated with acute renal allograft rejection $(407,423,535)$ and with chronic rejection of heart (193, 226, 277, 319), lung (281) and liver transplants $(20,292,379)$.

Although CMV infection is though to be a risk factor for chronic renal rejection as shown in animal studies $(209,228)$, little is known about the role of CMV in chronic rejection of renal allografts in humans. The histological diagnosis of chronic kidney allograft rejection in humans is well defined and consists of focal interstitial lymphocytic inflammation and fibrosis, glomerular mesangial matrix increase and sclerosis, vascular intimal proliferation and tubular atrophy $(391,392)$. End-stage chronic rejection is predominantly characterized by interstitial fibrosis.

A positive correlation between CMV and the development of chronic renal rejection has been reported in animals $(293,294,526)$. In the model used by Lautenschlager et al., rats received an allogeneic kidney transplantation under triple drug immunosuppression, which consisted of azathioprine, methylprednisolone and cyclosporin (483). Rats were subsequently challenged with RCMV or were mock infected. Viral antigens could be demonstrated in endothelial cells of capillaries and arterioles of RCMV infected animals. In contrast, mock-infected animals demonstrated characteristic histological changes of chronic rejection 60 days after transplantation, whereas those changes could already be demonstrated in RCMV-infected animals at 20 days after transplantation. The accelerated development of chronic rejection was associated with an increased and prolonged inflammatory response (294).

In liver transplantation, CMV infection has been associated with late acute rejection (82) and chronic rejection (vanishing bile duct syndrome, VBDS) $(147,292,380)$. The role of CMV infection in acute liver allograft rejection was studied in a rat model (329), which was developed by the group of Lautenschlager (330). CMV was shown to significantly increase portal inflammation and caused more severe bile duct damage when compared to uninfected control rats during acute liver allograft rejection (329).

Additional rat studies have shown a positive correlation of CMV and solid organ rejection in lung ${ }^{(506)}$, heart $(302)$, aorta $(309,310)$ and tracheal $(274,276)$ transplantation. 
Several mechanisms for the CMV mediated enhancement of solid organ rejection have been postulated. Graft failure due to viral or immune-mediated destruction of infected cells, or activation of the immune system by CMV, which mounts an additional inflammatory response to the alloresponse, has been suggested in this matter. Evidence supporting the latter hypothesis is increasing (230).

The process of inflammation is crucial for organ transplant rejection. Extravasation of leukocytes into the allograft is mediated by an intimate interaction of leukocytes with endothelial cells. Firm adhesion of leukocytes to endothelial cells is mediated by binding of intercellular adhesion molecule-1 (ICAM-1) and vascular adhesion molecule-1 (VCAM-1), both members of the $\mathrm{Ig}$ superfamily, to the integrin molecules, lymphocyte functionassociated antigen-1 (LFA-1) and very late antigen-4 (VLA-4), expressed on leukocytes $(493,494)$. CMV infection has been shown to enhance adhesion molecule expression on endothelial cells in vitro $(199,455)$ as well as in vivo $(24,84,275,329,505,506,513,557)$. Induction of expression of adhesion molecules is mediated by cytokines, such as IFN-gamma, TNFalpha and interleukin-1 (IL-1) (494). It was shown in vitro by Waldman et al., that CMV infected endothelial cells were able to activate host T cells. Production of IFN-gamma and TNF-alpha by these T cells was responsible for the observed enhancement of adhesion molecule expression on endothelial cells (541).

The role of MHC class I and II antigens in the inflammatory process leading to organ transplant rejection has also been subject of several studies. CMV has been shown to enhance MHC class II in vivo (535) and $\mathrm{MHC}$ class I in vitro, using cultured tubular epithelial cells (528) and endothelial cells (527). Upregulation of MHC molecules by CMV could not be demonstrated in human umbilical vein endothelial cells ${ }^{(456)}$ and in human kidney cells of tubular, glomerular or endothelial origin ${ }^{(524)}$. However, it should be noted that the virus interferes with MHC class I (546) and class II (354) molecule expression and that viral homologs of MHC class I (39) and class II (173) molecules have been identified in the genome of CMV.

\section{Treatment of cytomegalovirus infection}

Compounds used for treatment of cytomegalovirus infections

Ganciclovir, 9-(1,3-dihydroxy-2-propoxymethyl)guanide (DHPG), is an acyclic analogue of the natural nucleoside 2-deoxyguanoside. After intracellular phosphorylation of ganciclovir to ganciclovir-triphosphate, the compound competes with deoxyguanoside triphosphate and this results in an inhibition of viral DNA synthesis. The antiviral activity of ganciclovir against CMV, HSV-I and HSV-II has been extensively evaluated. Ganciclovir also inhibits replication of VZV, EBV, human adenovirus and HHV-6. Ganciclovir is used for treatment of $\mathrm{CMV}$-associated syndromes, such as retinitis, prevention and treatment of CMV disease in transplant recipients, CMV pneumonia, CMV-induced esophago- 
gastointestinal infections and CMV hepatitis. The most frequent side effect of ganciclovir treatment is bone marrow depression (especially neutropenia). Ganciclovir treatment has also been associated with impairment of CNS function, abnormal serum liver protein values and allergic reactions, such as fever and rash $(157,378)$.

Foscarnet, trisodium phosphonoformatic acid (PFA), is a pyrophosphate analogue. Foscarnet inhibits non-competitively and binds reversibly to the DNA polymerases of herpesviruses, including CMV (100). The compound reduces CMV replication significantly in vitro as well as in vivo. Foscarnet is used for the treatment of CMV retinitis (systemical as well as intra-ocular administration of the drug) and gastro-intestinal CMV infection. The major side-effects of foscarnet therapy, are the relatively short half-life of the drug which necessitates intravenous administration of the drug, renal function impairment, disturbances in serum calcium and phosphate levels and anemia. Renal impairment is reversible when foscarnet administration in ceased (236).

Cidofovir is a acyclic analogue of the corresponding monophosphate of deoxycytidine. The compound possesses a phosphonomethylether moiety that is resistant to metabolism. After diphosphorylation, the compound competitively competes with incorporation of deoxycytosine-5'-triphosphate. This process decreases drastically the efficiency of viral DNA transcription (299). Cidofovir shows a broad spectrum antiviral activity against DNA viruses, including papillomaviruses, poxviruses and herpesviruses (118). Cidofovir has been shown to be a potent inhibitor of CMV replication in vitro ${ }^{(372,373)}$ as well as in vivo ${ }^{(287,374 .}$ 498, 500). Cidofovir is mainly used for the treatment of CMV retinitis.

Despite the potent antiviral properties of the drug, clinicians should be aware of the following disadvantages. Firstly, in contrast to intravenous infusion of the compound, oral administration is characterized by a low bioavailability (539). Secondly, the major adverse effect of cidofovir is nephrotoxity and as a consequence usage is limited in patients with renal function impairment (261). After intravenous infusion, cidofovir is excreted unchanged over $90 \%$ in the urine ${ }^{(118)}$. Clearance of cidofovir exceeds the glomular filtration rate, indicating that tubular excretion is involved. It has been shown that accumulation of the drug within the proximal tubulus cells results in a dose limiting toxicity. However, administration of probenicid, a drug which inhibits active renal excretion, decreases cidofovir induced nephrotoxity (119). This also suggests a direct relationship between tubular accumulation of cidofovir and nephrotoxity. Minor adverse effects are neutropenia and uveiitis. 
Acyclovir, 9-(2-hydroxyethoxymethyl)guanine, is a nucleotide analogue, with minimal in vitro activity against CMV (110). Therefore clinical usage is restricted. It has been reported that high dose of acyclovir could have a prophylactic effect in transplant recipients at risk for CMV infection $(131,143,178)$. However, it should be noted that not all authors were able to confirm these findings $(27,189,266,463,553)$.

Antisense oligonucleotides are synthetic, short, single-stranded sequences of DNA or RNA that are designed to target and bind to messenger RNA. This attachment disrupts gene expression and inhibits protein synthesis. Fomivirsen is a novel antisense phosphorothioate oligonucleotide, which binds to major IE1 and IE2 mRNA of CMV. However, the observed antiviral effect of the compound in vitro $(23,130)$ cannot be explained by an antisense mechanism alone, since sequence-independent inhibition of virus absorption to host cells was also observed (17). In addition, intra-vitreal injection of fomivirsen decreased CMV activity in previously untreated patients suffering from CMV retinitis as well as in patients with CMV retinitis which was refractory to treatment with established anti-CMV compounds (127, 396). Although the results of fomivirsen administration for $\mathrm{CMV}$ retinitis are promising, clinical experience and application are limited. Unfortunately, there are several side-effects of intra-vitreal administration of the drug such as increased ocular pressure, bull's-eye pigmentary changes in the macula and mild to moderate intra-ocular inflammation of the anterior and posterior compartments of the eye $(15,127,508)$.

\section{Treatment of CMV infections in AIDS patients}

Ganciclovir (158), foscarnet $(153,462,542)$ and cidofovir (288)(2) have successfully been used in the treatment of CMV disease in AIDS patients. Before HAART therapy became available, the incidence of CMV retinitis was reported up to $85 \%$ in AIDS patients. The potent inhibition of HIV replication by HAART, resulted in a significant increase in CD4 count. Restoration of immune competence of the immune system enabled effective control of CMV infections.

Initial treatment of CMV retinitis in AIDS patients consists of intravenous (i.v.) ganciclovir or i.v. foscarnet plus HAART in HAART-naive patients (375). If CMV retinitis occurs in a patient who is already receiving HAART, high dose of i.v. ganciclovir, sometimes in combination with intra-ocular treatment may be used $(333,375)$. With progression of retinitis, intra-ocular treatment should be considered (375). In case of optimal HAART-related immune-reconstruction, oral ganciclovir $(1,134,488)$ or even cessation of antiCMV treatment may be optional.

Intra-ocular injections of foscarnet (375), ganciclovir (107), cidofovir (177, 263) and fomivirsen (127, 396, 403) have been effective in patients with a decrease in visual acuity due to CMV despite HAART or i.v. anti-CMV compounds. The intra-ocular ganciclovir implant (332) has 
been shown to be superior to all other intra-ocular therapies ${ }^{(375)}$. However, local therapy does not prevent systemic disease and/or progression into bilateral acuity loss (107, 334, 365). These patients may benefit from oral ganciclovir (333).

\section{Treatment of cytomegalovirus infections in transplant recipients}

Hematopoietic stem cell transplantation has been associated with a high incidence of and severe outcome of disseminated CMV disease, especially in CMV seropositive patients. Prevention of CMV infection in seronegative- and CMV recurrence in seropositive recipients can be achieved by using CMV seronegative or leukocyte-depleted blood products $(66,425)$. In addition, seropositive recipients or seronegative patients receiving bone marrow from a seropositive donor should receive prophylactic or pre-emptive antiviral therapy $(375,425,507)$. Prophylactic treatment consists of treating all patients at risk, whereas pre-emptive treatment is based on laboratory evidence of viral replication, such as viral culture, pp65 antigenemia and detection of viral DNA. Pre-emptive treatment reduces unnecessary drug exposure and allows immunological recovery $(62,142,317)$.

CMV pneumonia is lethal in $80 \%$ of the patients and high dose of i.v. immunoglobulins in addition to ganciclovir have been shown to improve survival (507), with a response rate of $60 \%$ and a long term survival of $30 \%(375,425)$. Usage of immunoglobulin infusions as monotherapy is controversial $(425,507,549)$.

CMV induced gastro-intestinal disease and CMV retinitis can be treated with i.v. ganciclovir or i.v. foscarnet. Intra-ocular treatment for CMV retinitis may be considered in affected eyes refractory to treatment.

A major drawback of ganciclovir treatment is the increased incidence of neutropenia in BMT patients (41-58\%) when compared to AIDS patients (34\%) and solid organ transplant patients $(10 \%){ }^{(444)}$. An alternative for ganciclovir is administration of a high dose of acyclovir $(351,408,409)$ or foscarnet $(232,318,427)$. Ganciclovir is the treatment modality of choice for treatment of CMV infection of solid organ transplant recipients (375). Initial treatment is usually given until active CMV replication has ceased. However, after cessation relapses are common and the incidence of recurrence has been estimated to be $25 \%{ }^{(449)}$. Current diagnostic tools for detecting CMV infection allow safe pre-emptive treatment in solid organ transplant recipients with a lower risk for CMV infection (seronegative donor I seropositive recipient or seronegative donor / seronegative recipient) (81, 388, 394, 463). Prophylactic therapy is particularly attractive for patients at a high risk for CMV infection, i.e. seronegative recipient receiving an allograft from a seropositive donor $(176,320)$. 


\section{Drug resistance}

The active metabolites of ganciclovir, cidofovir and foscarnet inhibit the DNA polymerase (UL18) of CMV. Phosphorylation of ganciclovir into its active metabolite form, depends on the UL97 gene product $(315,511)$. In contrast to ganciclovir, cidofovir does not require viral gene products for its activation into cidofovir-diphosphate (101). Foscarnet is an direct non-competitive inhibitor of the UL54 gene product (100).

Resistance to ganciclovir is associated with mutations in UL97 alone (30, 98, 152, 551, 552, 558) or in combination with mutations in UL54 $(468,510)$. Mutations in UL54 alone are rare. Resistance to foscarnet occurring during first line treatment is seldom (307) and occurs predominantly after extensive ganciclovir treatment $(97,448,468)$.

Recently, also CMV strains were isolated which showed resistance to cidofovir (95, 99, 140, 206). Also, treatment of in vitro CMV infected cells yielded viral strains which were less sensitive to the drug $(102,475)$.

Isolation of multiple resistant viral strains has important clinical implications and may result in uncontrollable deterioration of the patient. Follow-up of antigenemia levels may be a guideline for suspecting infection with resistant CMV strains. A decrease in antigenemia levels during anti-viral therapy indicates a good response of the virus to the drug. In case of increasing antigenemia values, a drug resistant CMV strain should be suspected (28).

\section{Rat cytomegalovirus as a model for cytomegalovirus infections}

RCMV (Maastricht strain) has been isolated from salivary glands extracted from wild brown rats (Rattus norvegicus) ${ }^{(78)}$. Infection of immunocompetent rats with RCMV resulted in a disseminated infection of liver, spleen, kidney, bone marrow and thymus at very low concentrations ( $<12$ plaque forming units $(\mathrm{PFU}) / \mathrm{ml}$ ), during the first week after infection $(75$. 77). From 1 week p.i. onward, the salivary gland yields high amounts of infectious virus (75). The amount of infectious virus in this organ depends on the rat strain used (75). Excretion of RCMV occurred predominantly via the saliva, although also low level of virus were found in the urine during the first weeks p.i. (77).

Infection of rats with irradiation-induced immunosuppression increases viral load in 'internal' organs, such as spleen, kidney, liver and lungs (497). In addition, irradiationinduced immunosuppression (5Gy) is required for lethal CMV infections (497).

Recently, the complete DNA sequence of the rat cytomegalovirus genome was described (531). The length of the genome was found to measure $229,896 \mathrm{bp}$. The RCMV genome encodes at least 166 protein-encoding open reading frames (ORFs), of which 113 and 76 have significant homology to ORFs of MCMV (Smith) and CMV (AD169), 
respectively. The RCMV genome encodes homologs of cellular proteins. In this regard, it is interesting that the RCMV genome encodes for homologs of $G$ protein coupled receptors (proteins R33 and R78), as well as for homologs of chemokines and MHC class I molecules (proteins r131 and r144) $(41,43,44,531)$.

The rat/RCMV model has been generally accepted as an appropriate model for studying CMV infections in humans based on its viral characteristics and biological behavior and has been used for studying the role of the virus in vascular pathology and in allograft rejection $(302,309,330,483)$. In addition, this model has also been used for elucidating pathogenesis of RCMV infection, especially in the immunocompromised host, and evaluation of novel antiviral compounds (497). 
Chapter 2

Rat cytomegalovirus (RCMV) replication in the salivary glands is exclusively confined to striated duct cells. 


\section{Summary}

The salivary gland is the preferent organ for cytomegalovirus replication and viral persistence. To identify the nature of infected cells and to study viral replication in more detail, several experiments were conducted. For this purpose, using the rat CMV (RCMV) model, acutely infected young adult rats (6 weeks of age) and new-born rats ( 3 days of age) were infected and submandibular, parotid and sublingual glands were harvested at different time points after infection. For identification of the nature of infected cells, immunohistochemistry, in situ hybridisation and electron microscopic techniques were used. In young adult animals, the submandibular gland was the preferent organ for RCMV replication. The parotid and sublingual gland, contained less virus. In contrast, in new-born rats the main site of RCMV replication was the sublingual gland while the submandibular and parotid gland contained low amounts of virus. In all glands of RCMV-infected animals, the infection was exclusively confined to striated duct cells. Infection resulted in a cellular inflammatory response which was mostly located in the interlobular duct region, whereas only few inflammatory cells were found in the neighbourhood of infected striated duct cells. This phenomenon may contribute to the long persistence of the virus in this organ. 


\section{Introduction}

Cytomegalovirus (CMV) is one of the most common viruses affecting humans. Infection of the immunocompetent host is usually associated with mild disease or asymptomatic infection. After primary infection the virus persists in its host in a latent state from which it can be reactivated as was shown for human CMV (CMV) $(54,55,73)$, murine CMV (MCMV) (419) and rat CMV (RCMV) (74) .

The rat model provides an appropriate model for CMV, since RCMV has a virion structure similar to CMV and the pathology it induces both in vitro as in vivo is similar to the CMV induced pathology $(76,410,497)$. Infection of immunosuppressed rats with RCMV results in a generalised infection with presence of infectious virus in most organs, such as liver, spleen, heart, kidney and lungs (497). In these organs infectious virus is already detectable at day 3 post infection (p.i.). Within these organs the virus is found in many cell types, among them brown fat cells, endothelial cells and monocytes/macrophages. After this period, the so called acute phase, which lasts for 1-2 weeks, no infectious virus is detectable in these organs. An exception is the salivary glands, where the virus persists for a long period i.e. several months up to one year p.i. $(75,77)$. The persistence of the virus in this organ is characteristic for RCMV and is believed to be on of the principal routes by which the virus is spread within the population of susceptible hosts. In summary, in the salivary glands, RCMV infection is characterised by two main properties: 1) infectious virus can be detected at a later time point than in all other organs, starting at day 7 p.i. and 2) infectious virus is produced in very high concentrations during a long period of time.

The tropism of this virus for the salivary glands and the long term shedding from this organ is one of the main characteristics of all CMVs. During evolution, the virus has been adapted to this target organ as shown by studies using deletion mutants of the virus ${ }^{44,}, 90$, 326, 446). In rats and mice infection with deletion mutants can result in a generalised infection with presence of virus in internal organs but without infectious virus and virus-infected cells in the salivary glands. This indicates that the virus harbours within its genome genes, that code for proteins/antigens which are necessary for infection of and replication in salivary glands.

Over the years many research groups have studied the course of CMV infection in both humans and animal models. Although the salivary gland is one of the main organs involved in replication of CMV and spread of the virus within the population, little is known of the cells that are infected and are producing infectious virus.

To study RCMV-infection of the salivary glands more thoroughly and to identify the nature of infected cells, we injected RCMV into the peritoneal cavity of young adult rats and followed the infection in the salivary glands. Since it is known that during the early phase of life organs are in development, we tested whether the age of the host is of importance for the infection of salivary gland cells. To study this, we included acutely 
infected new-born animals in this study and followed the infection in their salivary glands.

\section{Materials and methods}

Animals, virus and immunosuppression

Inbred male specific pathogen free (SPF; according to ${ }^{(422)}$ ) Lewis rats were used in all experiments. The animals were purchased from the Department of Experimental Animal Service at the University of Maastricht, the Netherlands. The animals were infected at the age of 3 days (new-born rats) or 6 weeks (young adult rats). The RCMV inoculum was derived from submandibular / sublingual salivary gland homogenate obtained as described previously (75) and was intraperitoneally (i.p.) administered at a dose of $1 \times 10^{4}$ and $1 \times 10$ ${ }^{5}$ plaque forming units (PFU), respectively (497). Negative salivary gland homogenate was used for mock infection. CMV infection is known to be enhanced by immunosuppression. Therefore, the 6 weeks old rats were immunosuppressed using 5 Gy total body irradiation (TBI) (497). New-born rats have an immature immune system and were considered as "naturally" immunosuppressed.

Prior to the experiments, permission was obtained from the Ethical Commission for Laboratory Animals of the University of Maastricht, Maastricht, The Netherlands and all experiments were performed according to the Principles of laboratory animal care $(\mathrm{NIH}$ publication No. 85-23, revised 1985) and the Dutch Law on Laboratory Animals.

\section{Experimental design}

The 6 weeks old animals were divided in three groups. The first group $(n=5)$ received immunosuppression and was subsequently infected with RCMV. A second group consisted of immunosuppressed mock-infected animals $(n=3)$, while a third group consisted of immunocompetent (non-irradiated) RCMV-infected rats $(n=3)$. The rats were sacrificed at 7, 14 and 28 days post infection (p.i.). New-born rats $(n=3)$ were infected at an age of 3 days and were subsequently sacrificed at 21 days p.i. At time of sacrifice, the submandibular, parotid and sublingual gland of young adult and new-born rats were sampled and processed for histological analysis and assessment of infectious virus. None of the infected rats showed symptoms of CMV disease as was defined previously (497).

\section{Immunohistochemistry}

Tissue samples were fixed in a phosphate buffered $3.7 \%$ formaldehyde solution in PBS for 24 hours and subsequently embedded in paraffin. RCMV-induced antigens were detected in $4 \mu \mathrm{m}$ tissue sections using a mouse monoclonal antibody (MoAb) 8 directed against an early nuclear RCMV antigen (79). For evaluation of the inflammatory response MoAb ED-1, W3/13 and 3.2.3. were used, with specificity for monocytes-macrophages (133), T lymphocytes (91) and NK cells (71), respectively. Positive cells were visualised using 
diaminobenzidine (DAB) substrate and slides were counterstained with haematoxilin-eosin. For inflammatory assessment, positive cells in 5 representative high power fields (HPF, $400 \times$ magnification) were counted. The presence of inflammatory cells was assessed semi-quantitatively. Absence of cells was scored as -; sporadic cells as \pm ; small numbers of cells as + ; considerable number of cells as ++ ; high numbers of cells +++ .

Images of tissue sections were analysed using a computerized morphometric analysis system (Qwin Software, Leica, Cambridge). Total cellular and nuclear area was assessed at 28 days p.i. in samples harvested from young adult animals as well as from new-born rats and were expressed as mean \pm SEM.

\section{In Situ Hybridisation (ISH)}

Detection of RCMV DNA was performed using the ISH technique as described previously ${ }^{(497)}$. In short, paraffin embedded sections $(4 \mu \mathrm{m})$ were dewaxed and proteolytic digestion was carried out $\left(5 \mathrm{~min}, 37^{\circ} \mathrm{C}\right)$ with pepsin $(2 \mathrm{mg} / \mathrm{ml})$. Denaturation $\left(80^{\circ} \mathrm{C}\right.$ for 5 min) was followed by hybridisation with the DNA probe overnight at $42^{\circ} \mathrm{C}$. The DNA probe consisted of the Xbal fragments C,D and E labelled with biotin 11-dUTP (343). Positive cells were visualised with DAB substrate. The ISH stained sections were used for viral load assessment since ISH is more sensitive than immunohistochemistry, as described before (196).

\section{Ultrastructural analysis by electron microscopy}

Rats were anesthesized by injection of $0.1 \mathrm{ml}$ Pentobarbital (6\%) / $100 \mathrm{~g}$ body weight intraperitoneally. Subsequently, rats were perfused with $0.1 \mathrm{M} \mathrm{Na}$-cacodylate buffer + 2\% PVP (MW 40.000) pH 7.4 for 3 minutes (flowrate $15 \mathrm{ml} / \mathrm{min}$ ), followed by perfusion fixation using fixative containing $4 \%$ Paraformaldehyde, $2 \%$ Glutaraldehyde and $0.2 \%$ Picrin-acid in $0.1 \mathrm{M} \mathrm{Na-cacodylate} \mathrm{buffer} \mathrm{pH}$ 7.4. After perfusion, salivary glands (submandibular, sublingual and parotid) were removed and fixed overnight using the same fixative. Thereafter, tissues were washed $0.1 \mathrm{M} \mathrm{Na}$-cacodylate buffer $\mathrm{pH} 7.4$ containing $6.8 \%$ sucrose $\left(3 \times 5 \mathrm{~min}\right.$.) followed by another $2 \mathrm{hr}$ fixation in $1 \% \mathrm{OsO}_{4}+1.5 \% \mathrm{~K}_{4} \mathrm{Fe}(\mathrm{CN})_{6}$ in $0.1 \mathrm{M} \mathrm{Na}$-cacodylate buffer at $4^{\circ} \mathrm{C}$. Again, tissues were washed in $0.1 \mathrm{M} \mathrm{Na}$-cacodylate buffer $\mathrm{pH} 7.4$ containing $6.8 \%$ sucrose $(3 \times 5 \mathrm{~min}$.) at room temperature and subsequently dehydrated in $30 \%, 50 \%$ and $70 \%$ EtOH (10 min. each) followed by $2 \mathrm{hr} 100 \% \mathrm{EtOH}$. Tissues were embedded in Epon 812 and semi-thin $(1 \mu \mathrm{m})$ tissue sections were stained using Toluidine Blue. Areas containing RCMV infected cells were selected by light-microscopy. Selected areas were processed for ultra-thin sectioning $(70 \mathrm{~nm})$ and ultra-thin sections were contrasted according to Reynolds, using $2 \%$ uranylacetate (15 min.) and leadcitrate (2 min.). Finally, TEM analysis was performed at $60 \mathrm{kV}$ using a transmission electron microscope (Philips, EM201, The Netherlands). 


\section{Assessment of infectious virus}

The amount of infectious virus was determined in salivary glands by plaque assay using rat embryonic fibroblasts (REFs) as described before ${ }^{(78)}$. In short, the organs were homogenised and suspended in minimal essential medium containing $2 \%$ fetal calf serum. Tenfold dilutions of $10 \%$ homogenates $(\mathrm{w} / \mathrm{v})$ were inoculated on confluent REF monolayers. After an incubation period of 7 days, under $0.25 \%$ agarose, the number of plaques was determined microscopically after fixation and methylene blue staining.

\section{Statistics}

For statistical analysis the two-tailed non-parametric Mann Withney $U$ test was used. Differences associated with $p<0.05$ were considered statistically significant. Data were presented as mean \pm SEM.

\section{Results}

Localisation of RCMV infection in the salivary glands of young adult rats

Infection of salivary glands was studied in young adult rats using several virological and histological techniques at 7, 14 and 28 days p.i. Presence of infectious virus in submandibular, parotid and sublingual glands was tested by plaque assay at 14 and 28 days p.i. The submandibular and parotid gland yielded high concentrations of infectious virus: $8.09 \pm 0.25$ and $4.98 \pm 0.22{ }^{10} \log$ PFU per gram tissue at 14 days p.i., respectively (table 1). Production of infectious virus increased in these organs during the course of infection, being, at 28 days p.i., $10.90 \pm 0.17{ }^{10} \mathrm{log}$ PFU for the submandibular and $8.54 \pm$ $0.40{ }^{10} \mathrm{log}$ PFU for the parotid gland. In contrast, the amount of infectious virus found in the sublingual gland decreased from $8.51 \pm 0.28{ }^{10} \mathrm{log}$ PFU at 14 days to $6.91 \pm 0.67{ }^{10} \mathrm{log}$ PFU at 28 days p.i. The number of infected cells within these glands was determined using the ISH technique and is expressed as number of positive cells per 1000 striated duct cells, as is shown in table I. The number of infected cells during the course of infection correlated accurately with the data from the plaque assays. Data from both techniques indicate that at 28 days p.i., the submandibular gland is the preferent organ for RCMV replication, while the parotid and the sublingual gland contained significantly lower concentrations of virus.

Immunohistochemistry and ISH revealed that infection was confined to striated duct cells in submandibular, parotid and sublingual gland of immunosuppressed young adult animals. Granular convoluted duct cells, acinar cells, intercalated duct cells and interlobular duct cells remained negative during the course of infection. 
Table 1.

Presence of RCMV in the salivary glands of 6 week old rats ${ }^{\text {a }}$

\begin{tabular}{lccc} 
Salivary glands & days p.i. & RCMV positive cells $^{b}$ & viral load $^{c}$ \\
\hline submandibular gland & 7 & $0 \pm 0$ & ND $^{d}$ \\
& 14 & $114 \pm 18$ & $8.09 \pm 0.25$ \\
parotid gland & 28 & $116 \pm 19$ & $10.90 \pm 0.17$ \\
& 7 & $0 \pm 0$ & $\mathrm{ND}$ \\
sublingual gland & 14 & $10 \pm 4^{\mathrm{e}}$ & $4.98 \pm 0.22^{\mathrm{e}}$ \\
& 28 & $55 \pm 24^{\mathrm{e}}$ & $8.54 \pm 0.40^{\circ}$ \\
& 7 & $0 \pm 0$ & $\mathrm{ND}$ \\
& 14 & $95 \pm 35^{\circ}$ & $8.51 \pm 0.28$ \\
& 28 & $53 \pm 21^{\circ}$ & $6.91 \pm 0.67^{\circ}$
\end{tabular}

\footnotetext{
a The rats received 5 Gy TBI before i.p. inoculation with $10^{5}$ PFU RCMV at the age of 6 weeks

b RCMV-infected cells were detected by in situ hybridisation (ISH) and the number of positive cells is expressed per 1000 striated duct cells (mean \pm SEM)

c Viral load was measured by plaque assay and expressed as log PFU/g tissue (mean \pm SEM)

d Not Determined

e Significant $(p<0.05)$ when compared to the submandibular gland
}

Infection of immunocompetent animals resulted in the same viral tropism for striated duct cells, although the concentration of infectious virus and virus infected cells was lower or even undetectable (data not shown).

Uninfected striated duct cells showed a rectangular shape $(17.22 \pm 0.13 \times 9.21 \pm$ $0.45 \mu \mathrm{m}$ ) with their small basis attached to the basal membrane. The cells contained a centrally located round nucleus $(7.40 \pm 0.13 \times 6.55 \pm 0.14 \mu \mathrm{m})$. At the basis of the cell multiple invaginations could be appreciated. The most prominent finding in RCMV-infected striated duct cells was the enormous increase in total cell size $(p<0.05$ when compared to non-infected) (figure 1A). Cellular and nuclear size assessment was conducted at 28 days p.i. The cytoplasm protruded into the lumen of the duct and in some cases multiple infected cells resulted in a partial or even complete obliteration of the duct. The cytoplasm was filled with granular material, which gave the cells a foam-like appearance. This increase in cytoplasmic volume resulted in a more basally located nucleus and disappearance of the invaginations. In contrast to non-infected striated duct cells, the 
nucleus was of oval shape $(16.04 \pm 0.64 \times 8.96 \pm 0.55 \mu \mathrm{m})$ and significantly enlarged (figure $1 \mathrm{~A}$ ). In the duct, infected cells are most frequently lined by non-infected cells (figure $2 \mathrm{~A}$ ) and infection was focally distributed throughout the gland.

In contrast to the submandibular gland, the parotid gland was characterised by a more lobular structure of the gland and the absence of granular convoluted duct cells. The sublingual gland also lacks this duct segment. In both glands infection was also confined to striated duct cells. There were no differences in morphology of infected cells between the submandibular, parotid and sublingual gland.

In addition to light microscopy, electron microscopy was used as a golden standard for identification of infected cells in the submandibular, parotid and sublingual gland. The observations found by light microscopy were confirmed by EM. Infected cells were characterised by an enlarged ovally shaped nucleus and by an increase in total cell size as described above. The surrounding cells are characterised by basal invaginations, the presence of many mitochondria and a centrally located nucleus indicating that the infected cells are part of the striated duct (figure 2A). In the nucleus of infected cells many immature virus particles could be visualised. Figure 2B suggests the budding of a mature virus particle from the nuclear membrane (arrowhead 8). Additionally, several relatively small vesicles containing mature and immature viral particles and dense bodies assemble in the cytoplasm. As depicted in figure 2D, the mature virus particles consist of a nucleocapsid with an electron dense DNA structure. The nucleocapsid is surrounded by tegument and viral envelope. The large vesicle fuses with the cellular membrane and releases its contents into the lumen (figure $2 \mathrm{C}$ ). In conclusion, RCMV replication in salivary glands of young adult Lewis rats is exclusively confined to striated duct cells as shown by immunohistochemistry, ISH and EM.

\section{Localisation of RCMV in the salivary glands of the newborn rat}

Preliminary data in our laboratory suggested that infected cells were of granular convoluted duct origin due to the characteristic appearance of infected cells. Data from young adult animals shown above suggested otherwise. To confirm our data from the young adult animals, a second model was employed which lacks the putative target cells. This model consisted of infection of newborn rats at the age of 3 days. The animals were sacrificed 21 days p.i., i.e. just before onset of granular duct development. As shown in table 2, infectious virus could be detected in the salivary glands of these animals, especially in the submandibular and sublingual gland. 

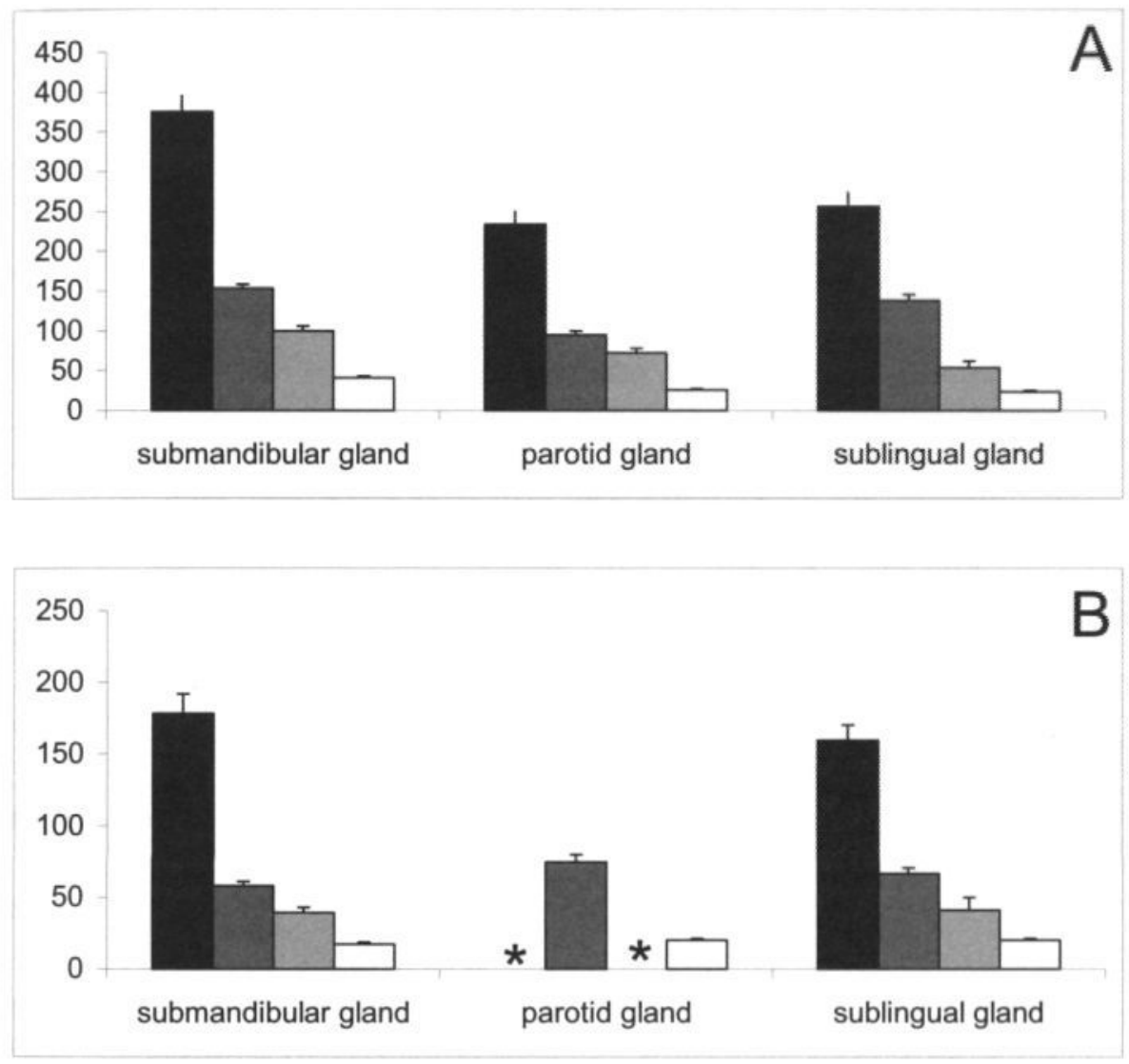

Figure 1. Total cell- and nuclear area of RCMV-infected (measured by ISH) and non-infected striated duct cells was assessed in the submandibular, parotid and sublingual gland from young adult (A) and new-born rats (B). Salivary glands harvested at 28 days p.i were used for assessment. (black bar) $=$ total cell area of RCMV-infected striated duct cells; (dark grey bar) = nuclear area of RCMV infected striated duct cells; (light grey bar) = total cell area of noninfected striated duct cells; (white bar) = nuclear area of non-infected striated duct cells. Data are expressed as mean \pm SEM $\left(\mathrm{mm}^{2}\right)$. All differences between infected and non-infected cells are significant $(p<0.05)$. By ISH, no viral DNA could be detected in parotid glands $\left(^{*}\right)$ extracted from new-born rats. 
Table 2.

Presence of RCMV in the salivary glands of new-born rats ${ }^{a}$

\begin{tabular}{lcc} 
Salivary glands & RCMV positive cells ${ }^{b}$ & Viral load $^{\mathrm{c}}$ \\
\hline Submandibular gland & $23 \pm 8$ & $5.6 \pm 0.5$ \\
Parotid gland & $0 \pm 0^{\mathrm{d}}$ & $3.1 \pm 0.4^{\mathrm{d}}$ \\
Sublingual gland & $66 \pm 17^{\mathrm{d}}$ & $7.0 \pm 0.3^{\mathrm{d}}$
\end{tabular}

rats were infected with $10^{4}$ PFU RCMV at day 3 after birth and were sacrificed at day 21 p.i.

b measured by in situ hybridisation (ISH) and expressed per 1000 striated duct cells (mean + SEM).

c Viral load was measured by plaque assay and expressed as ${ }^{10} \mathrm{log}$ PFU/g tissue (mean \pm SEM)

d Significant when compared to the submandibular gland $(p<0.05)$

In contrast to young adult animals, the amount of infectious virus and the number of RCMV-infected cells in new-born animals was significantly higher in the sublingual gland than in the submandibular glands while low concentrations of infectious virus, measured by plaque assay but no viral DNA, using ISH, was found in the parotid gland of these animals. In concert with their young adult counterparts, viral replication was restricted to striated duct cells and similar cytopathology of the infected cells was found (figure 1B). In conclusion, these experiments in new-born rats show that despite differences in salivary gland tropism, RCMV replication in these animals is also confined to striated duct cells and indeed independent of granular convoluted duct cells.

\section{Inflammatory cell influx in the RCMV-infected salivary glands}

RCMV infection of the salivary glands of young adult rats generated a cellular inflammatory response as shown by the presence of monocytes/macrophages, lymphocytes and NK cells at day 14 and 28 post infection (table 3 ). At these time points the number of inflammatory cells in the submandibular and in the parotid glands was significantly higher than in the glands of mock-infected animals. In the sublingual glands of the infected rats, no increased influx of inflammatory cells was observed compared to controls. The influx of monocytes/macrophages preceded the influx of T lymphocytes in the submandibular and the parotid glands. A similar observation was reported previously by Persoons et al. (399) using the subcutaneously-infected hind paw model.

The influx of NK cells was rather low and was maximal at 2 weeks p.i. (table 3). This is of importance since it has been suggested that especially NK cells are responsible for the clearance of $\mathrm{CMV}$ infection $(52,544)$. 

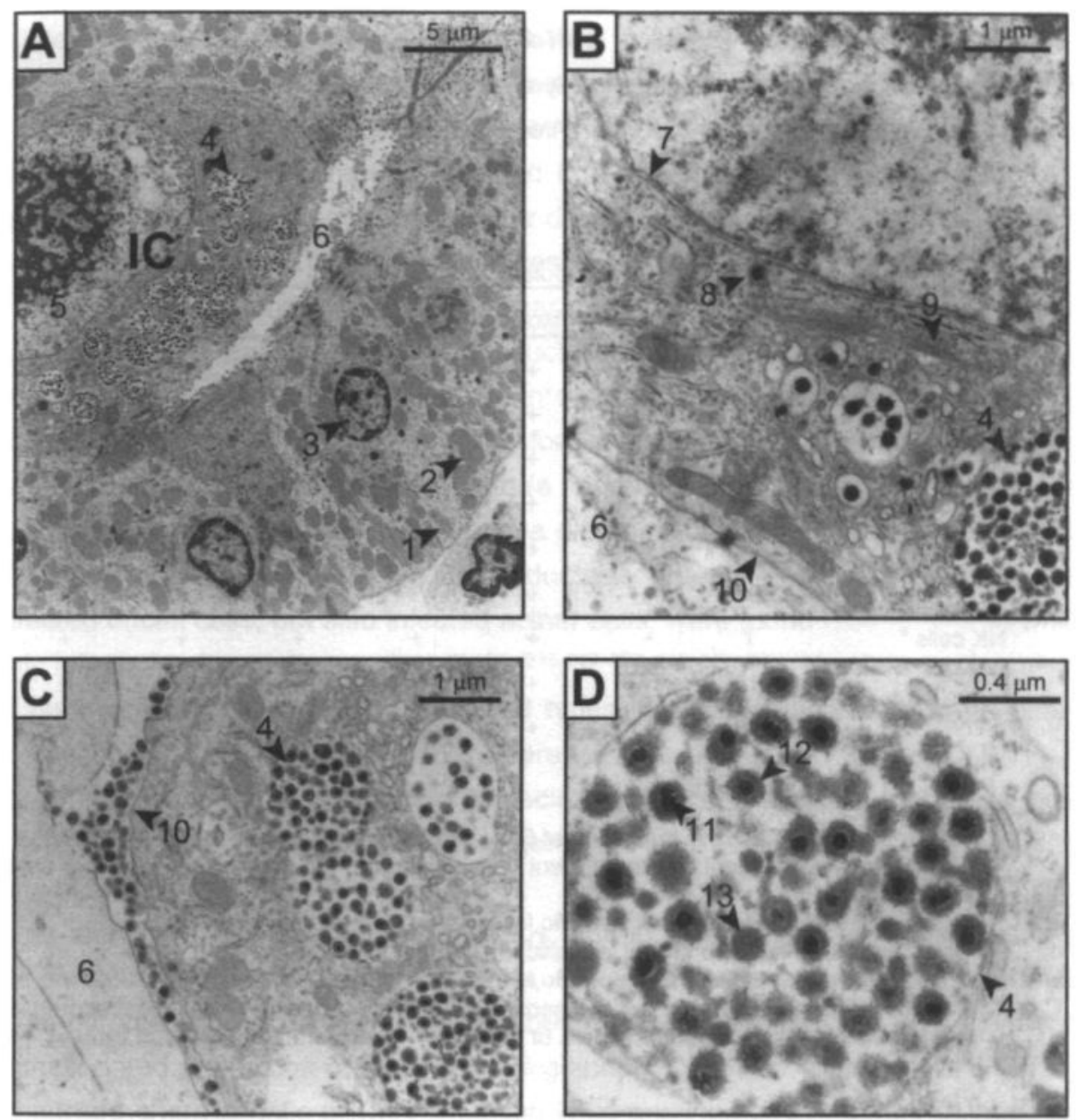

Figure 2. Striated duct cells are characterised by basal striations, a centrally located nucleus and many mitochondria. The infected cell (IC) is located within the striated duct (A). Assembly of small vescicles containing mature and immature viral particles at the Golgi apparatus is shown in figure B. The mature virus particles consist of a nucleocapsid with an electron dense DNA structure (D). The nucleocapsid is surrounded by tegument and viral envelope. Release of virus particles in the lumen of the duct is shown in figure C. Arrowheads indicate the following structures: 1 = basal striations; 2 = mitochondrium; 3 = uninfected nucleus; 4 = virus containing vesicle; $5=$ RCMV-infected nucleus; $6=$ lumen; $7=$ nuclear membrane; $8=$ budding of a virus particle from the nuclear membrane; $9=$ Golgi apparatus; $10=$ cellular membrane; $11=$ nucleocapsid; 12 = tegument and viral membrane; 13 = dense body. 
Table 3.

Presence of monocytes/macrophages, T-lymphocytes and NK cells in the salivary glands of RCMV-infected rats ${ }^{\text {a, b }}$

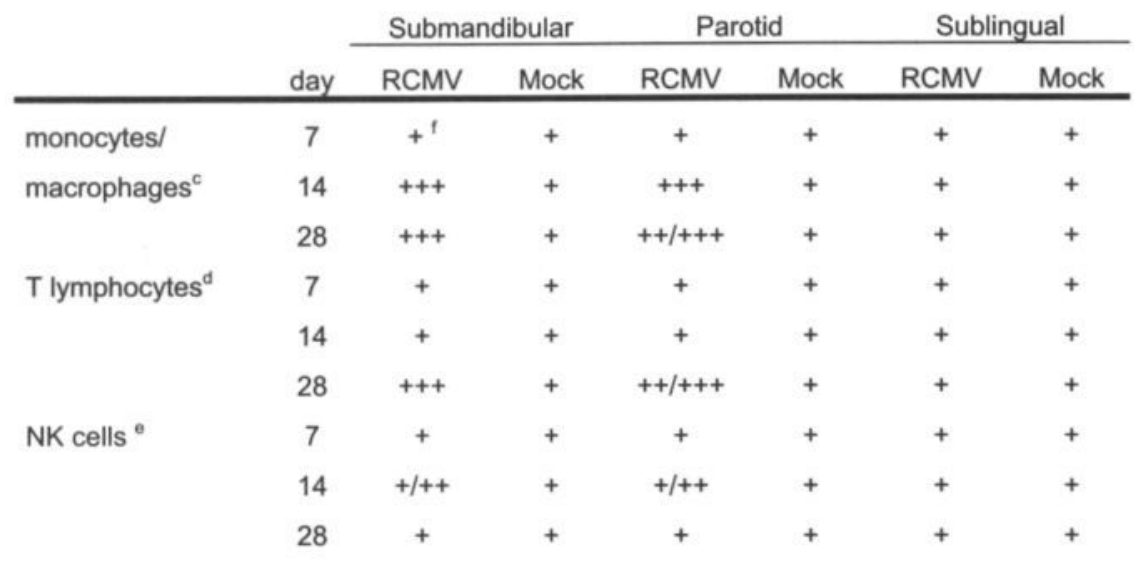

a Rats received a total body irradiation of $5 \mathrm{~Gy}$ before inoculation with $10^{5} \mathrm{PFU}$ RCMV.

b The animals were sacrificed at different time points and the salivary glands were used for this study.

c Cells reactive with MoAb ED-1, specific for monocytes/macrophages

d Cells reactive with MoAb W3/13, specific for T lymphocytes

Cells reactive with MoAb 3.2.3, specific NK cells

The presence of cells was assessed semi-quantitatively. Absence of cells was scored as $-;$ sporadic cells as +; small numbers of cells as +; considerable number of cells as ++; high numbers of cells +++

Another interesting finding in this study was that inflammatory cells detected in the infected glands were not localised around the infected striated duct cells, as expected (figure $3 \mathrm{~A}$ ), but were confined to the interlobular duct region in the submandibular and parotid gland (figure 3B). This was true for both immunocompetent as immunosuppressed animals (data not shown). Only few inflammatory cells were present in the sublingual gland (table 3). This is an intriguing observation since it could be one of the mechanisms involved in the persistence of infected cells in this organ.

\section{Discussion}

In this study a detailed description of CMV replication in the submandibular, parotid and sublingual salivary gland of the rat is reported. The main objective of the present study was to assess the identity of the type of RCMV-infected cells in the organs. Initially, preliminary data suggested that infected cells were of granular convoluted duct origin due 
to the characteristic appearance of the infected cells. By using different techniques (immunohistochemistry, in situ hybridisation and electron microscopy) it became clear that not the granular convoluted duct cells but the striated duct cells were infected. This finding was further supported by the results obtained in intraperitoneally infected newborn rats, which were sacrificed before onset of granular duct development (at 4 weeks of age). Our study clearly shows that RCMV replication was exclusively confined to striated duct cells in all three salivary glands and that acinar cells, intercalated duct cells, granular convoluted duct cells and the interlobular duct cells remained free of virus. This is of interest, since striated duct cells differentiate into granular convoluted duct cells starting at 4 weeks of age (242) and as a result the ability for RCMV to replicate in these cells is lost in the process. Restriction of CMV replication to (a segment of) ductal cells in the rat model is in concert with the data obtained in the guinea pig infection model using guinea pig CMV (GPCMV), in which infection was limited to ductal segments $(49,164,165,196)$. In humans, CMV infected ductal cells, but also infected acinar cells, were found (194, 402, 471, 540), while in mice only infected acinar cells are described (215-217, 227, 248, 356, 382). No clear explanation for these differences in infection of different cell types in salivary glands between different models has been given. Especially, the difference between RCMV and MCMV in their behaviour for salivary gland cell tropism is fascinating since it is known that these viruses possess a lot of similarities and that the genomes of both viruses show the highest homology of all known CMVs $(42-44,47)$.

Another interesting observation of this study is that, although RCMV replicates only in the striated duct cells in the salivary glands, the virus content is significantly different among the different glands, suggesting that other yet unexplained factors are also involved in the infection and production of virus in these glands.

An important characteristic of CMV infection is its persistence in salivary glands. In the rat model persistent infection accompanied by continuous shedding of virus from this organ has been shown earlier (77). The cells responsible for this persistence and shedding are striated duct cells, since only these cells contain high amounts of virus particles, as shown by immunohistochemistry, ISH and electron microscopy.

From our in vivo data and from results obtained with other cells, such as endothelial and smooth muscle cells, we know that at least two factors contribute to persistent infection; first the type of cell that is infected and secondly the immune response of the host to this infection. Depending on the type of cell that is infected, an infection results in cell death (fibroblasts ${ }^{(78)}$ ), an abortive infection (endothelial cells, ${ }^{(57)}$ ) or a persistent infection (smooth muscle cells and monocytes- macrophages (162, 521). In the present study we detected not only that striated duct cells are the preferent cells for CMV infection in the salivary glands but also that in these cells the production of virus particles follows a different course than in fibroblasts. 

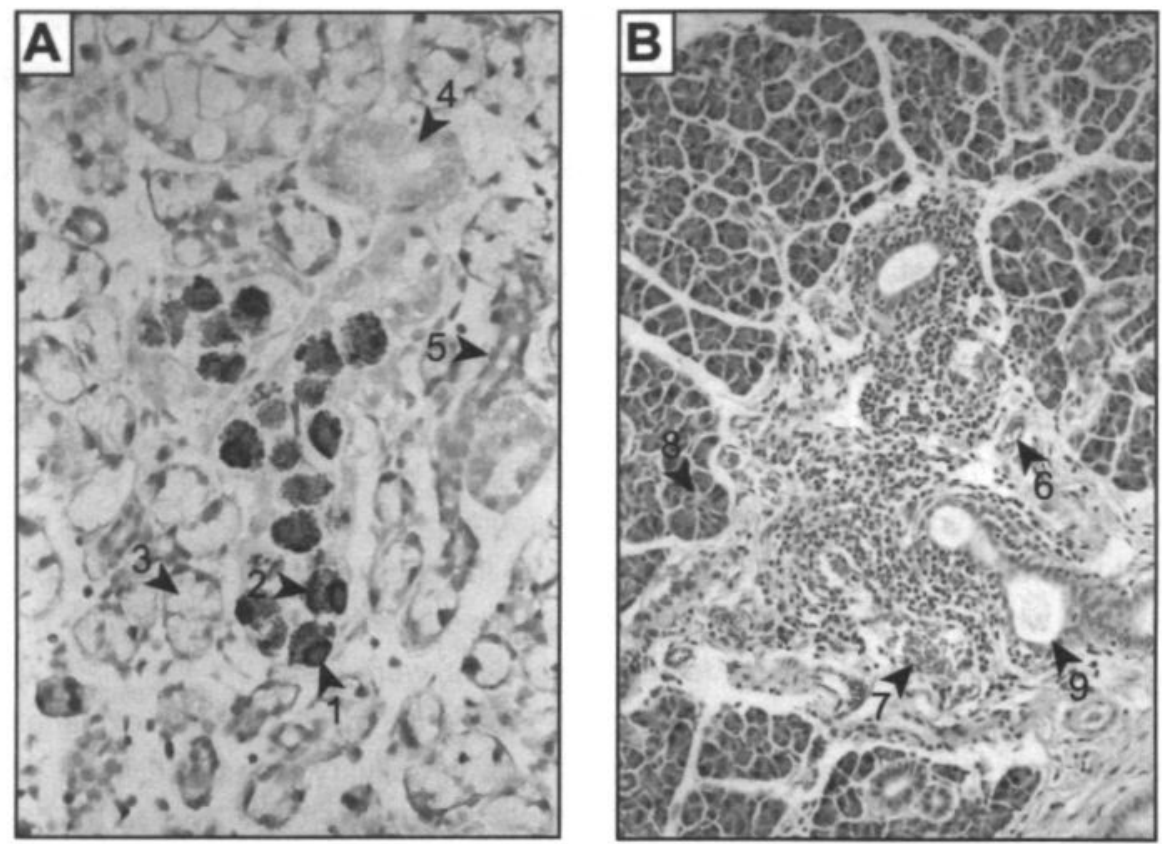

Figure 3. A) Detection of viral DNA by ISH in the submandibular gland of young adult rats at 28 days p.i. The nuclear staining of RCMV-infected cells (arrowhead 1) can be distinguished from the cytoplasmic staining of viruscontaining vesicles (arrowhead 2). Note the relative absence of inflammatory cells. B) Parotid gland of a young adult rat at 28 days p.i. Note that the influx of inflammatory cells is predominantly confined to the interlobular duct region. Arrowheads indicate the following structures: $3=$ acinus; $4=$ uninfected striated duct segment; $5=$ intercalated duct segment; $6=$ artery; $7=$ vene; $8=$ acinus; 9 = interlobular duct.

While in fibroblasts, as in neo-intimal smooth muscle cells, high concentrations of virus particles were found in the nucleus $(397,519)$, we detected large amounts of virions in vacuoles in the cytoplasm of striated duct cells. Such observations have thus far not been performed in other cell types of rat origin. It suggests that replication, production and excretion of the virus in striated duct cells is different from replication in fibroblasts and is cell type dependent. However, it should be noted that virus containing vesicles is a common observation in the human system (359).

In vivo the situation is even more complex since not only the cell type is important but also other factors such as the immune response of the host. In our study it was found that in the infected salivary glands, inflammatory cells were not localised directly around the infected cells but were localised at the interlobular duct regions. This is an interesting 
finding since it might explain the persistence of $\mathrm{CMV}$ in salivary glands. A similar observation has also been described for the mouse model (216) indicating that, in contrast to other CMV infected organs, the inflammatory cells in the salivary glands do not reach the infected cells. Thus they are not able to eliminate virus infected cells and by this way limit the infection. The reason for this remains unknown. However, it is known that eventually the virus is removed from the gland (75). In addition, electron microscopy showed that viral excretion from the infected cell is by apical cell budding of the larger virus containing vesicles. It should be noted, however, that transmission of virus to other cells via direct cell-cell contact could not be excluded by electron microscopy. A possible mechanism for viral clearance from these organs might be that infected cells can only infect downstream-located striated duct cells. During the course of infection, infected cells located in the proximal striated duct segment would die and would be replaced by noninfected counterparts. As a result, the infection would be removed from the gland in the direction of saliva flow, without intervention of the immune system. Adding to this is the fact that the number of striated duct cells decreases with aging of the rat due to the differentiation of striated duct cells into granular convoluted duct cells (242).

Also other mechanisms could be involved in the persistence of the infection in the salivary glands, for example the observation that, in contrast to other organs, the NK cell influx in this organ is only slightly enhanced, while in other infected organs a high influx of NK cells is found (525). Since it is known that these cells are important cells for the clearance of CMV infected cells ${ }^{(458)}$, diminished influx of NK cells could play a role in persistence of the virus in the salivary glands.

In conclusion, we showed that RCMV exclusively infects striated duct cells in the salivary glands. In these cells, high levels of infectious virus are produced during a long period p.i. Persistence in the salivary glands is partly due to the type of cell that is infected, but probably also to a failure of the immune reaction in removing the virus-infected cells in these organs. The difference between MCMV and RCMV in preference of infection site is an interesting observation that cannot yet be explained. The use of in vitro cell culture in the near future will give us more answers about infection and persistence of CMV in salivary glands.

\section{Acknowledgements}

The authors would like to thank Joanne van Dam and Timi Martelius for carefully reading the manuscript. 
Chapter 3

Persistent rat cytomegalovirus (RCMV) infection of the salivary glands contributes to the anti-RCMV antibody response. 


\section{Summary}

The salivary glands are the major sites of persistent replication of rat cytomegalovirus (RCMV). At several months post infection (pi), infectious RCMV is usually still produced in the salivary glands but not in any other organ or tissue of the rat. To investigate whether the persistence of RCMV in the salivary glands is crucial to the pathogenesis of viral infection, we monitored the progression of RCMV-induced disease in rats from which the salivary glands were surgically removed (desalivated) as well as in sham-operated rats, both after a lethal and sublethal challenge with RCMV. Interestingly, desalivation did not have a significant effect on either RCMV-induced morbidity or mortality. As expected, at one year pi, relatively high levels of infectious virus were detected in the salivary glands of sham-operated rats, whereas neither infectious virus nor RCMV DNA could be detected in liver, spleen and lungs of these animals. Infectious virus and viral DNA were also undetectable in organs from desalivated animals at one year pi. Interestingly, a difference was found between desalivated and sham-operated rats in the titers of anti-RCMV lgG antibodies, which were significantly higher in sham-operated rats than in desalivated animals at 183, 295 and 365 days pi. This finding indicates that the persistence of RCMV in the salivary glands may contribute significantly to the anti-RCMV humoral immunity of infected rats. 


\section{Introduction}

Cytomegalovirus (CMV) infection is common in the human population and seroprevalence has been reported to be as high as $80-90 \%$. In immunocompetent individuals, infection is usually asymptomatic. However, when the host's immune system is compromised (i.e., in AIDS patients or transplant recipients), CMV infection may cause life-threatening disease and even lead to death $(210,400)$.

In our laboratory, a rat cytomegalovirus (RCMV)/rat model has been developed in order to study the pathogenesis of CMV infections $(75,77,78)$. RCMV infections in this model can be characterized by two distinct phases. In the first phase, during the first week post infection (pi), a generalised infection of internal organs, such as liver, kidney, pancreas, lung and spleen occurs ${ }^{(497)}$. Although viral DNA can be detected in the salivary glands by $\mathrm{PCR}$, neither infectious virus nor viral antigens are found in these glands during the first phase of infection $(42,43)$. The second phase of infection initiates at approximately 8 days pi. During this phase, the salivary glands start to produce high levels of infectious virus for a prolonged period of time $(75,77)$. The infection of the salivary glands can therefore be regarded as a persistent infection. By contrast, infectious virus can no longer be detected in 'internal' organs after the first week pi, whereas viral DNA can occasionally be detected (43). Thus, RCMV may reside in these organs in a latent form.

In the rat salivary glands, RCMV replication was found to be confined to striated duct cells of the submandibular, parotid and sublingual glands (267). Within these cells, virus particles are deposited in large cytoplasmic vesicles, which release their contents in saliva by apical cell budding (267). The virus-containing saliva is generally regarded as a crucial vehicle for horizontal transmission of RCMV. However, the production of high virus titers in the salivary glands and saliva for longer periods of time could also have other consequences. It can be envisaged that the continuous production and accumulation of virus particles in the salivary glands may have an influence on the overall physiology of the infected host. Shedding of virus from the salivary glands and virus-containing saliva to other sites of the body may result in re-infection of internal tissues and organs, providing a continuous challenge for the host's immune system.

In order to study the role of the salivary glands in the pathogenesis of RCMV infection, we employed an experimental infection model in which viral replication and dissemination were monitored in rats in which the salivary glands had been surgically removed prior to infection. It has previously been demonstrated that desalivation of rats only results in relatively mild alterations in the normal physiology of the animals $(69,367,376,469)$. Here, we describe two separate experiments. In the first experiment, the effect of desalivation on RCMV-induced disease and mortality was studied after administration of a lethal dose of RCMV. In the second experiment, desalivated rats, sham-operated rats as well as mockinfected rats were challenged with a sublethal dose of RCMV. 


\begin{tabular}{|l|}
\hline Experimental \\
groups: \\
- Desalivated RCMV \\
infected rats $(n=7)$ \\
- Sham-operated \\
RCMV \\
infected rats $(n=7)$ \\
- Desalivated mock \\
infected rats $(n=7)$
\end{tabular}
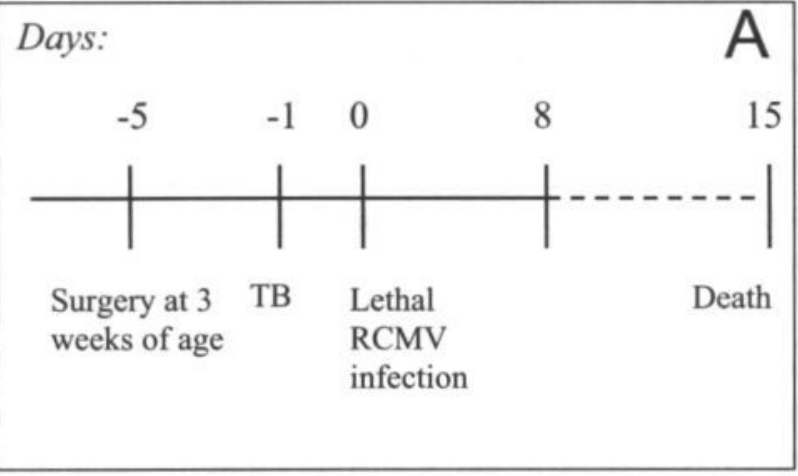

$$
\begin{aligned}
& \text { Experimental } \\
& \text { groups: } \\
& \text { - Desalivated RCMV } \\
& \text { infected rats }(n=8) \\
& \text { - Sham-operated } \\
& \text { RCMV } \\
& \text { infected rats }(n=8) \\
& \text { - Mock infected } \\
& \text { control rats }(n=3)
\end{aligned}
$$

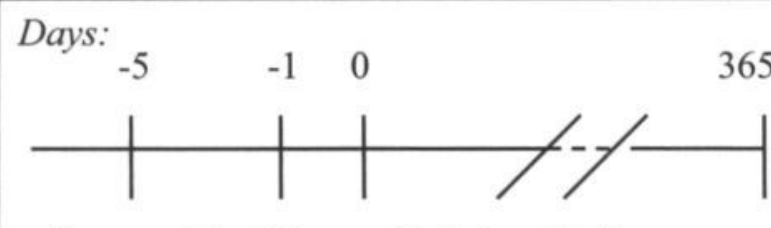

$\begin{array}{llll}\begin{array}{l}\text { Surgery at } 4 \\ \text { weeks of age }\end{array} & \text { TBI } & \begin{array}{l}\text { sublethal } \\ \text { RCMV } \\ \text { infection }\end{array} & \begin{array}{l}\text { Various } \\ \text { blood } \\ \text { taps }\end{array}\end{array}$

Figure 1. Schematic representation of the experimental design of the study. (A) Mortality experiment. The effect of desalivation on mortality after a lethal RCMV challenge was studied. (B) Long-term follow-up experiment. Rats were sublethally challenged with RCMV. The general condition of the animals was recorded. Blood samples were taken from the tail vein at 14, 29, 84, 117, 183, 245, 295 and 365 days p.i. in order to determine anti-RCMV IgG levels. At 1 year pi, various organs were harvested for detection of infectious virus and viral DNA.

Subsequently, the generation of anti-RCMV antibodies was measured during the course of infection until one year pi. In addition, the presence of infectious virus as well as viral DNA was assessed in various organs. Surprisingly, we found significantly higher titers of antiRCMV antbodies in sham-operated rats than in desalivated animals, indicating that persistently infected salivary glands may contribute to the anti-RCMV humoral immunity of infected rats. 


\section{Materials and Methods}

Animals and virus

Specific pathogen-free, inbred male Lewis rats were obtained from the Experimental Animal Facilities of the University of Maastricht, Maastricht, The Netherlands. The RCMV (Maastricht strain) inoculum was derived from submandibular and sublingual salivary gland homogenates, which were generated as described previously (497). Salivary gland homogenates from uninfected rats were used for mock infection.

\section{Extirpation of salivary glands}

Salivary glands were removed from either 3- or 4-week-old rats. Rats were anaesthesized with a mixture of ketamine $(100 \mathrm{mg} / \mathrm{kg}$, ip) and xylazine $(10 \mathrm{mg} / \mathrm{kg}$, subcutaneously (sc)). Both drugs were purchased from Eurovet (Bladel, The Netherlands). After a transverse incision, the submandibular, sublingual and parotid glands of the rats were removed and the skin was subsequently closed. An equal number of rats received a sham operation. Buprenorphine $(0.5 \mathrm{mg} / \mathrm{kg}, \mathrm{sc} ;$ Schering-Plough, Maarssen, The Netherlands) was used to alleviate post operative pain (313). All animals were fed acidified tap water and moistened, grinded rat chow (Hope Farms, Woerden, The Netherlands) ad libitum. The rat chow was suspended $(600 \mathrm{~g} / \mathrm{l})$ in $2 \%$ granulated agar (Becton, Dickinson and Company, Cockeysville, MD, USA) and enriched with sugar $(12 \mathrm{~g} / \mathrm{l})$. All rats quickly recovered and started to gain weight within 2 days after surgery. After 3 months, all rats returned to a diet of normal (ungrinded) rat chow ad libitum.

\section{Experimental design}

To study the influence of the salivary glands on the course of RCMV infection, two experimental models were employed (Fig. 1). In the first model (Fig. 1A), the effect of salivary gland extirpation on mortality after a lethal RCMV challenge was studied. Three weeks-old rats were subjected to a radical salivary gland extirpation. 

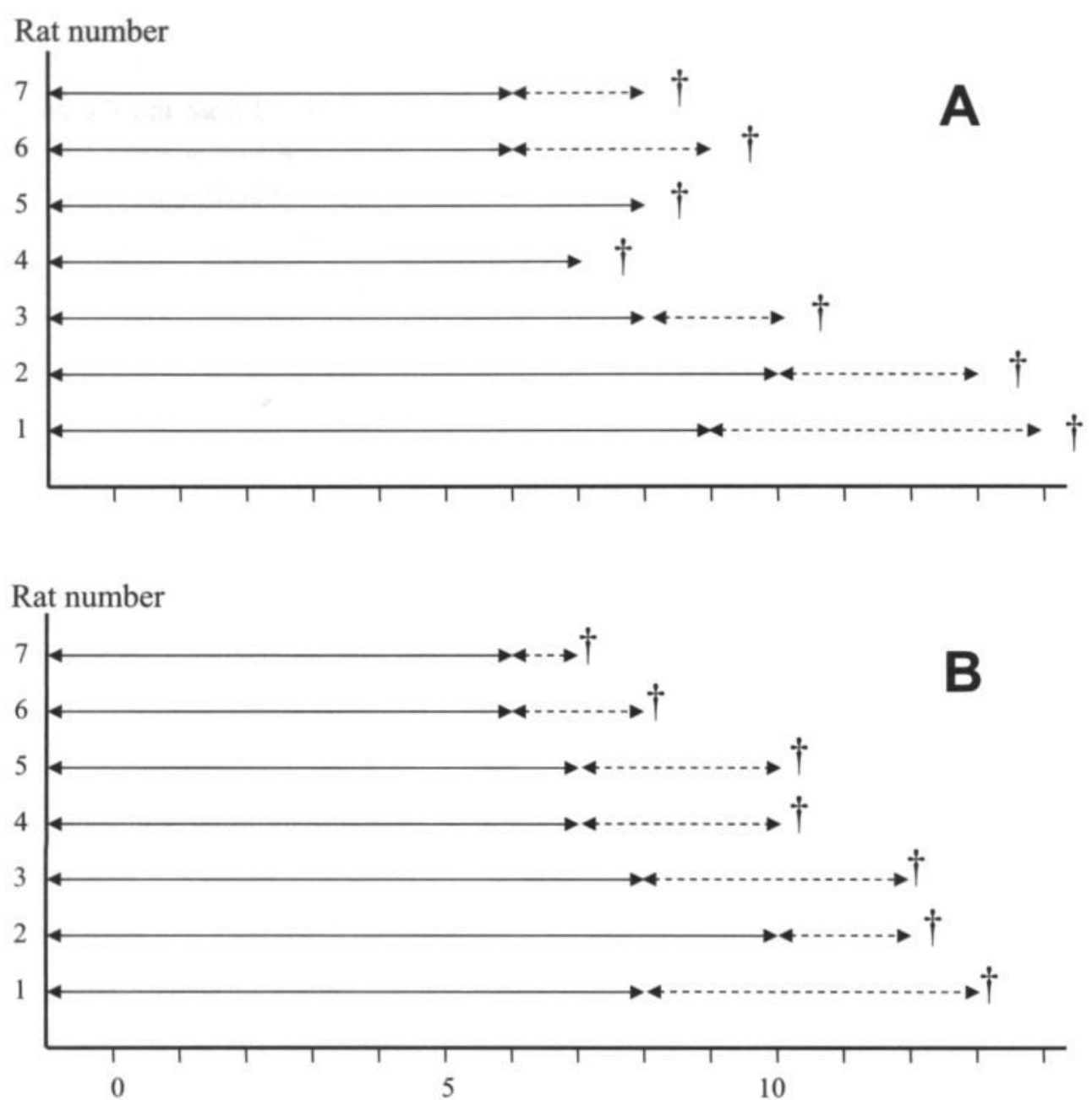

Figure 2. The onset of RCMV-induced disease and mortality in groups $(n=7)$ of RCMV-infected, sham-operated (A) and desalivated rats (B). The dashed lines represent the period in which symptoms of RCMV infection could be appreciated. $\dagger$ indicates time of death. The experiment was carried out as outlined in figure $1 \mathrm{~A}$.

Subsequently, at one day before infection (day -1), rats were immunocompromised by total body X-ray irradiation (TBI, 5 Gy). At day $0, \mathrm{RCMV}$ was administered intraperitoneally (ip) at a dose of $1 \times 10^{5}$ plaque forming units (PFU) (497). RCMV-infected, desalivated rats ( $n=$ 7) were compared to RCMV-infected, sham-operated animals $(n=7)$. Desalivated, mockinfected rats $(n=7)$ were used as control animals. Rats were checked for CMV disease (497) and weighed on a daily basis. In addition, survival was recorded. 
The second experimental model (Fig. 1B) consisted of a long-term follow-up of RCMV infection in salivary gland-extirpated rats and sham-operated rats as well as in mockinfected control animals. In this model, salivary glands were removed from 4 weeks-old rats $(n=8)$. Another subgroup of rats $(n=8)$ received sham surgery. Control rats were mock-infected and were not subjected to surgery $(n=3)$. After a recuperation period of 4 days, rats were irradiated and infected, similarly as described above. The rats were weighed daily, and the general condition of the animals (weight, dental health, behavior and condition of oral mucosa) was recorded. In order to assess anti-RCMV IgG levels, blood samples were taken from the tail vein at 14, 29, 84, 117, 183, 245, 295 and 365 days pi. At 1 year pi, organs (submandibular glands, liver, spleen and lung) were harvested for detection of infectious virus (by plaque assay) and viral DNA (by PCR).

\section{Detection of anti-RCMV IgG antibodies}

Blood samples were centrifuged for $10 \mathrm{~min}$ at $4500 \times \mathrm{g}$ and stored at $-20^{\circ} \mathrm{C}$ until use. An enzyme-linked immunosorbent assay (ELISA) was developed to monitor total anti-CMV $\lg \mathrm{G}$ in rat serum. To prepare the antigen, rat embryo fibroblasts (REF) were cultured in minimal essential medium (MEM) containing $10 \%$ newborn calf serum (NCS). The cells were infected with RCMV at a multiplicity of infection (MOI) of 0.05 in MEM containing $2 \%$ NCS. After an incubation period of 4 hours at $37^{\circ} \mathrm{C}$, the medium was replaced with fresh MEM containing $2 \%$ NCS and the cells were cultured until all cells showed cytopathic effect (CPE). The medium and the cells were collected and centrifuged for 10 minutes at $900 \times \mathrm{g}$ to remove cell debris. PEG 6000 was added to the supernatant at a concentration of $15 \%(\mathrm{w} / \mathrm{v})$ and the solution was stirred at $4^{\circ} \mathrm{C}$ for 4 hours. After centrifugation at $1400 \mathrm{x}$ $\mathrm{g}$ for 10 minutes, the pellet was resuspended in dialysis buffer $(10 \mathrm{mM}$ Tris- $\mathrm{HCl}, 1 \mathrm{mM}$ EDTA, pH 7.1) and dialyzed overnight at $4^{\circ} \mathrm{C}$. The solution was placed on a solution of $30 \%$ sucrose in dialysis buffer and centrifuged for 75 minutes at $1400 \mathrm{~g}$ at $4^{\circ} \mathrm{C}$. The pellet was diluted in dialysis buffer and stored at $-20^{\circ} \mathrm{C}$ until use. As control antigen, mockinfected cells were processed. The ELISA was performed in 96-well vinyl assay plates. The wells were washed with coating buffer $\left(2.5 \mathrm{mM} \mathrm{NaH} \mathrm{PO}_{4} \cdot \mathrm{H}_{2} \mathrm{O}, 7.5 \mathrm{mM}\right.$ $\mathrm{Na}_{2} \mathrm{HPO}_{4} .2 \mathrm{H}_{2} \mathrm{O}, 0.15 \mathrm{M} \mathrm{NaCl}, \mathrm{pH} 7.2$ ) and incubated overnight at $4^{\circ} \mathrm{C}$ with $0.1 \mathrm{ml}$ of antigen diluted $1: 10$ in coating buffer. After three washes with washing buffer $(2.5 \mathrm{mM}$ $\mathrm{NaH}_{2} \mathrm{Po}_{4} \cdot \mathrm{H}_{2} \mathrm{O}, 7.5 \mathrm{mM} \mathrm{Na}_{2} \mathrm{HPO}_{4} .2 \mathrm{H}_{2} \mathrm{O}, 0.5 \mathrm{mM} \mathrm{NaCl}, 1 \mathrm{mM}$ Tween 20, pH 7.2), $0.1 \mathrm{ml}$ of 1:10 diluted rat serum was added to each well. After incubation for 1 hour at room temperature (RT), the wells were washed and biotinylated, sheep anti-rat IgG (Amersham Pharmacia Biotech, Roosendaal, The Netherlands) was added, followed by another incubation step of $1 \mathrm{~h}$ at RT. Subsequently, the wells were washed and incubated with peroxidase-conjugated streptavidine (Amersham Pharmacia Biotech). 


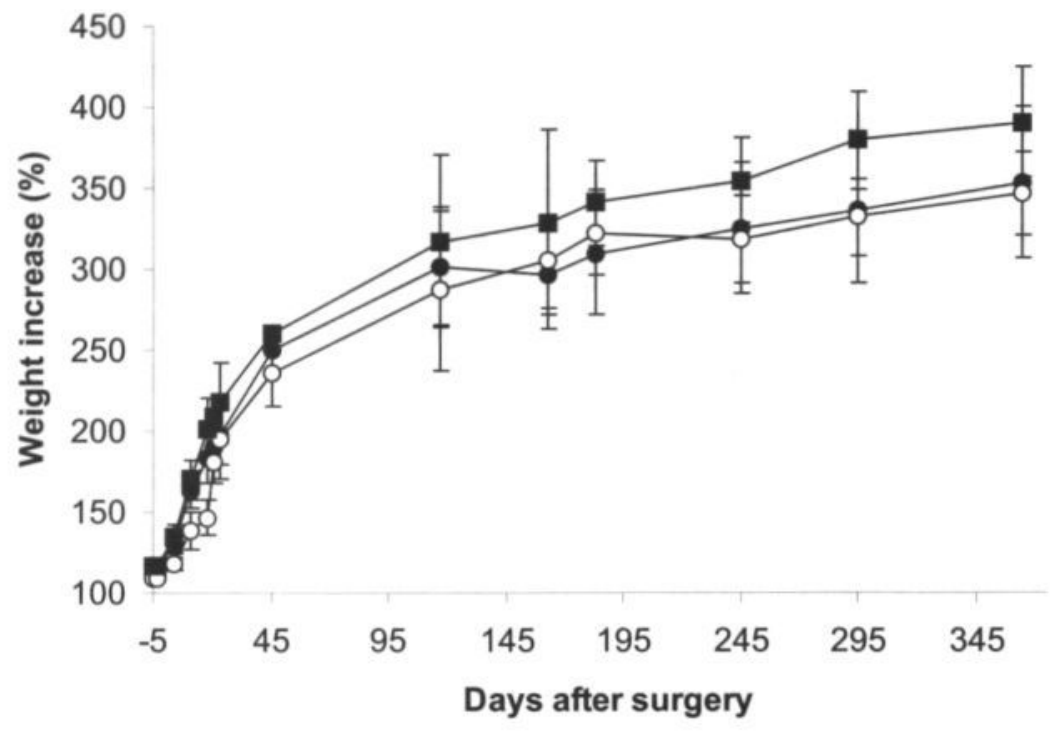

Figure 3. Relative weight increase during long-term follow-up of groups of RCMV-infected shamoperated (closed circels) rats, RCMV-infected desalivated rats (open circels) and untreated and non-infected (closed squares) animals. Experiments were conducted as outlined in figure 1B. Data are expressed as mean \pm SEM.

Immediately before use, the substrate solution was prepared (12 $\mathrm{mg}$ O-phenyldiamine and $15 \mu \mathrm{l} 30 \% \quad \mathrm{H}_{2} \mathrm{O}_{2}$ added to $36 \mathrm{ml}$ substrate buffer $\left(35 \mathrm{mM} \mathrm{C}_{6} \mathrm{H}_{8} \mathrm{O}_{7} \cdot \mathrm{H}_{2} \mathrm{O}, 67 \mathrm{mM}\right.$ $\mathrm{Na}_{2} \mathrm{HPO}_{4} \cdot 2 \mathrm{H}_{2} \mathrm{O}, \mathrm{pH} 5.0$ )), and $0.1 \mathrm{ml}$ was added to each well. The plate was incubated in the dark for 10 minutes and the reaction was stopped by adding $0.1 \mathrm{ml}$ of $4 \mathrm{M} \mathrm{H}_{2} \mathrm{SO}_{4}$. The extinction at $492 \mathrm{~nm}$ was measured using an ELISA reader (340 ATCC). All samples were processed simultaneously and each ELISA was performed in duplicate. The extinction values of the control-coated wells were subtracted from the extinction values of RCMV antigen-coated wells.

\section{Plaque titration assay}

The amount of infectious virus was determined in salivary glands by plaque assay as described previously ${ }^{(78)}$. In short, the organs were homogenised and suspended in MEM containing $2 \%$ NCS. Tenfold dilutions of $10 \%$ homogenates $(\mathrm{w} / \mathrm{v})$ were inoculated on confluent REF monolayers. After an incubation period of 7 days under $0.25 \%$ agarose, the number of plaques was determined microscopically, after fixation and staining with methylene blue. 


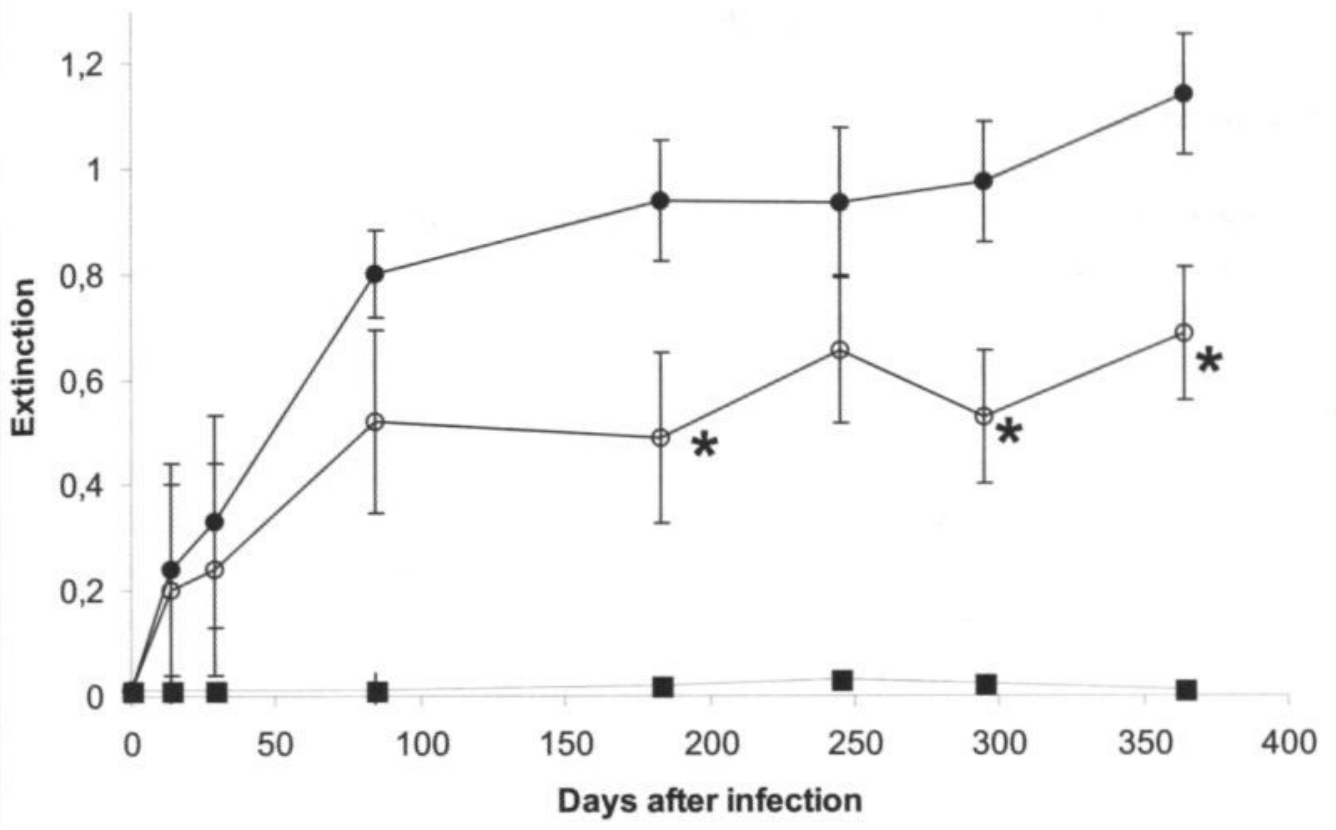

Figure 4. RCMV antibody response of sham-operated (black circle), desalivated (open circle), and control (black square) rats. Sham-operated and desalivated rats were irradiated (5 Gy TBI) and infected with $1 \times 10^{5}$ PFU of RCMV at day -1 and day 0 , respectively. Rats were sacrificed at 365 days p.i. Differences marked with * were considered to be statistically different when compared to sham-operated rats. Data are expressed as mean \pm SEM.

\section{RCMV PCR}

Organ samples were minced mechanically and DNA was isolated by using the Wizard® Genomic DNA Purification Kit (Promega, Madison, WI, USA). During the isolation procedure, organ homogenates were incubated with proteinase $\mathrm{K}$ at $60^{\circ} \mathrm{C}$ for 60 to 90 minutes. Viral DNA was detected by using a sensitive, single-tube, nested PCR which amplifies a part of the major immediate-early region of the RCMV genome. The PCR was carried out as described previously (43). In short, the PCR mixtures, with a total volume of $50 \mu \mathrm{l}$, contained $1 \mu \mathrm{g}$ of target DNA, $0.05 \mu \mathrm{M}$ of primers RIE3F (5'-CCA GAG TGA CGT TGC AGA TGT TGG AAA TCA-3') and RIE3R2 (5'-GGT CAC GAC CCT GCT GCC GTC TAG GT-3'), $1 \mu \mathrm{M}$ of each primer RIE4F (5'-ATG AAA TGG TGA TGA GAT-3') and RIE4R (5'-CTT CTA GTG ATT TGG CAT-3'), $100 \mu \mathrm{M}$ concentrations of each dNTP, $15 \mathrm{mM}$ $\mathrm{MgCl}_{2}$, HotStar Taq DNA polymerase buffer and $1.25 \mathrm{U}$ of HotStar Taq DNA polymerase (Qiagen, Leusden, The Netherlands). Amplification was performed with a GeneAmp PCR System 9600 thermal cycler (Perkin-Elmer, Nieuwerkerk aan de IJssel, The Netherlands). PCR products were visualized by ethidium bromide-staining after gel electrophoresis. 
Samples were tested both undiluted and 1:10 diluted. In addition, samples were tested with or without spike (10 copies of viral DNA).

\section{Statistical analysis}

For statistical analysis, the two-tailed non-parametric Mann Withney $U$ test was used. Differences associated with $p<0.05$ were considered statistically significant. Data are presented as mean \pm SEM.

\section{Results}

Desalivation does not have an influence on RCMV-induced disease and mortality

To study the role of the salivary glands in the pathogenesis of RCMV infection, we employed an experimental model in which the course of the infection was compared between groups of desalivated rats and normal, sham-operated rats. In an initial experiment (Fig. 1A), we compared the mortality rates of these groups of animals after infection with a lethal dose of RCMV. As shown in Fig. 2, there is no significant difference in mortality rate between desalivated rats and sham-operated animals. In both groups, the rats died between day 8 and 15 pi. In most animals, symptoms of RCMV-induced disease, such as mucosal haemorrhages, development of ascites and weight loss, were found approximately 3 days before death (Fig. 2). These symptoms, which have been described previously (497), appeared in a similar fashion in either group of animals. As a control, mortality was also investigated in a group of desalivated, mock-infected animals. As expected, each of the animals of this group survived the surgical procedure and did not have a mortality significantly different from that of untreated, mock-infected rats (data not shown). In addition, desalivation did not lead to detectable side effects in any of these animals. Taken together, our data indicate that desalivation does not have a significant effect on either onset or course of a lethal RCMV infection.

\section{Desalivated rats have a significantly lower titer of anti-RCMV antibodies than normal rats}

It was previously shown that desalivation does not result in significant alterations in the physiology of rats $(69,367,376,469)$. Nevertheless, in a second, long-term experimental model, in which rats were subjected to sublethal doses of RCMV (Fig. 1B), we monitored the health status of the animals on a daily basis, by using the following parameters: weight increase, dental health, the condition of the oral mucosa and behavior. At the time of surgery, the weights of the RCMV-infected, desalivated rats, RCMV-infected, shamoperated animals and mock-infected control rats did not differ significantly $(117 \pm 11 \mathrm{~g}, 133$ $\pm 13 \mathrm{~g}$ and $109 \pm 8 \mathrm{~g}$, respectively). During long-term follow-up, the weight of the animals increased steadily. However, significant differences in relative weight increase between the 
three experimental groups were not found (Fig. 3). In addition, differences in either dental health, oral mucosa or behavior could not be detected between these groups (data not shown). Thus, in accordance with previous reports $(69,367,376,469)$, our results indicate that surgical removal of the salivary glands does not have a significant influence on either growth or health status of the animals up to one year after surgery.

To investigate the dissemination of RCMV in desalivated rats after long-term infection, these animals, as well as RCMV-infected, sham-operated rats, were sacrificed at one year pi. Subsequently, the presence of both infectious virus and viral DNA was investigated in liver, spleen, lungs and submandibular glands (of sham-operated rats). As expected, infectious virus was detected in the salivary glands of sham-operated animals $\left(3.6 \times 10^{4} \pm\right.$ $2 \times 10^{3} \mathrm{PFU} / \mathrm{g}$ tissue), but not in any other tissue of either desalivated or sham-operated rats. This finding is in agreement with previous reports in which RCMV replication at several months after infection was detected exclusively in the salivary glands $(43,75,77)$. In order to detect viral DNA in the rat organ samples, we employed a previously described single-tube, nested PCR assay, which can detect 1 to 10 copies of RCMV DNA per $2.4 \mathrm{x}$ $10^{5}$ cells ${ }^{(43)}$. Previously, we could occasionally detect RCMV DNA in organs samples from RCMV-infected rats at 330 days pi (43). We therefore anticipated that viral DNA might also be found in some of the organ samples from rats sacrificed at 365 days pi. However, RCMV DNA was exclusively detected in the salivary glands of sham-operated animals (data not shown). The apparent discrepancy between this result and that of the previous study ${ }^{(43)}$ may be due to the use of a 10 times lower RCMV inoculum in the present study. Nevertheless, our results underline the importance of the salivary glands as the predominant site of long-term, persistent RCMV replication.

As described previously, an anti-RCMV IgG antibody response is elicited during longterm, persistent RCMV infection in rats (75). To investigate whether persistent RCMV infection of the salivary glands has an effect on this humoral immune response, we regularly collected serum samples over a one-year period from both desalivated and sham-operated, RCMV-infected animals. Subsequently, the presence of IgG antibodies directed against RCMV virion proteins in the rat sera was monitored by using an in-house developed ELISA (see Materials and Methods). Fig. 4 shows that the anti-RCMV IgG titers in sera from sham-operated animals are similar to those reported for normal, RCMVinfected rats in previous studies ${ }^{(75)}$. By contrast, significantly lower antibody titers were found in desalivated rats than in sham-operated animals at days 183, 295 and 365 pi. This finding indicates that the RCMV-infected salivary glands stimulate the production of antiRCMV antibodies. An explanation for this phenomenon may be that the continuous production of viral proteins and virions in the salivary glands provides a constant trigger, either directly or indirectly, to the immune system. Since this constant trigger is absent 
from desalivated rats, these animals may eventually develop a less efficient humoral immune response than normal RCMV-infected rats.

\section{Discussion}

The salivary glands are the preferred sites for persistence of CMVs (222). The persistent viral shedding via saliva over a long period of time is characteristic for all CMVs and is believed to play a major role in horizontal transmission of these viruses. In order to study the role of the salivary glands during the course of RCMV infection of rats, we employed an experimental animal model in which the pathogenesis of infection was compared between normal rats and rats from which the salivary glands were surgically removed (desalivation). Previous reports demonstrated that desalivation does not result in significant changes in the physiology of rats. Nevertheless, desalivation was found to result in delayed wound healing after surgical infliction of large ( 3 to $5 \mathrm{~mm}$ ) circular wounds in the palate (58-60). In addition, desalivation was reported to alter eating patterns and food preference of the animals $(69,367,376,469)$. It is important to note, however, that in previous studies with desalivated rats, different methods of desalivation were employed. In some cases, the desalivation procedure only consisted of ligation of the parotid duct, whereas the parotid gland was left in situ (58-60). However, in the present study, we relied on radical desalivation in which the submandibular, sublingual and parotid glands were surgically removed. This approach was chosen since RCMV is able to replicate in each of these glands (267). Following desalivation, the course of RCMV infection was monitored both after a lethal and sublethal challenge with RCMV. After a lethal challenge with RCMV, we were unable to observe significant differences between desalivated and normal, sham-operated rats, both in disease progression and mortality induced by the virus. However, after a sublethal challenge with RCMV, significantly higher titers of anti-RCMV IgG were found in sera from sham-operated rats than in those from desalivated rats. These differences in titers appeared at relatively late times after infection (183, 295 and 365 days pi). On the basis of these observations, we suggested that the RCMV-infected salivary glands augment the production of anti-RCMV IgG antibodies. However, the mechanism by which this augmentation is effectuated is currently unknown. It is possible that the anti-RCMV IgG titers are higher in sham-operated rats than in desalivated rats because the immune system of the sham-operated rats is constantly 'boosted' as a consequence of the relatively high level of replication of RCMV in the salivary glands. It was previously demonstrated that RCMV particles are collected in large cytoplasmic vesicles of striated duct cells and that the virions are released into the lumen of the duct via apical cell budding of the vesicles (267). Re-infection may subsequently occur, possibly via small lacerations, of the oral/pharyngeal and/or esophageal mucosa. Shedding of virus or viral 
proteins into the circulation, either directly from the salivary glands or from virus-containing saliva, may account for boosting of the humoral immune response.

In general, viral infections are controlled by both the cellular and humoral immune response $(426,432)$. The importance of the humoral immune response in the control of RCMV-induced disease has previously been demonstrated. Treatment of RCMV-infected, immunosuppressed rats with hyperimmune serum provided full protection against virusinduced mortality (497). Similarly, anti-HCMV antibodies were found to prevent severe HCMV disease in newborns (169), AIDS patients ${ }^{(13)}$, solid organ transplant recipients (298) and allogeneic bone marrow transplant recipients ${ }^{(453)}$. The protective effect of antibodies was also demonstrated in the murine CMV/mouse model system (154, 250,459). In conjunction with these previous reports, the present study indicates that persistent infection of the salivary glands by RCMV may have at least two major consequences. First, the production of infectious virus by the salivary glands, and subsequent shedding of infectious virus in the saliva, provides an important means for the virus to spread horizontally through the population. Second, the high level production of viral proteins and particles may boost the humoral immune response of the infected host and thereby protect the host against repeated challenges by either endogenous or exogenous RCMV.

In conclusion, the productively RCMV-infected salivary glands contribute significantly to the formation of anti-RCMV IgG antibodies. Viral shedding from this organ or from viruscontaining saliva may be responsible for boosting the anti-RCMV antibody response, which may subsequently protect the host from either endogenous or exogenous reinfections. The elucidation of the mechanisms that underly the augmentation of the antiRCMV humoral immune response will be the challenge of future studies.

\section{Acknowledgements}

The authors would like to thank Peter Kelderman for the excellent surgical procedure, Rien Blok for help with the PCR assays and Sabine Peters for expert technical assistance. C.V. is supported by a fellowship from the Royal Netherlands Academy of Arts and Sciences (KNAW). 
Chapter 4

A rat cytomegalovirus strain with a disruption of the r144 MHC class I-like gene is attenuated in the acute phase of infection in neonatal rats. 


\section{Summary.}

We previously generated an RCMV strain in which the r144 gene, encoding a major histocompatibility complex class I homolog, had been deleted (RCMV $\Delta r 144$ ). To investigate the role of r144 during acute infection of neonatal rats, we infected three daysold neonatal rats with either RCMV $\triangle$ r144 or wild type (wt) RCMV and the presence of infectious virus as well as viral DNA in various organs was determined at either 3, 5 or 21 days p.i. In addition, we assessed both type and number of inflammatory cells in these organs. Interestingly, a significantly lower concentration of infectious virus as well as viral DNA was found in spleens of RCMV $\triangle$ r144-infected rats than in those of wt RCMV-infected animals at 3 days p.i. At the same time point, a significantly lower amount of infiltrating NK cells and monocytes/macrophages was seen in the spleens of RCMV $\Delta$ r144-infected rats than in spleens of rats infected with wt RCMV. At 21 days p.i., RCMV $\Delta$ r144-infected rats were found to have lower virus titers in the salivary glands than wt RCMV-infected animals. No significant differences between RCMV $\Delta$ r144- and wt RCMV-infected rats were detected at other time points nor at other sites. We conclude that after infection of neonatal rats, the replication of RCMV $\triangle \mathrm{r} 144$ is severely restricted compared to wt RCMV. 


\section{Introduction}

Cytomegalovirus (CMV) is an important human pathogen causing severe disease in the immunosuppressed host. AIDS patients, transplant recipients and especially newborns are prone for severe HCMV disease. Viral shedding is common during early childhood and may persist for two years or more (384). Thereafter the virus persists in the host in a latent state $(64,203,212,271,272,466)$. The ability to enter latency is a characteristic shared by all CMVs. Immune-evasive strategies of HCMV are likely to play an important role in the process of latency and currently several virus-encoded immune-modulators were identified (reviewed by S. Michelson (353)).

One of the strategies of human CMV (HCMV) to evade immune surveillance is the downregulation of cellular MHC class I molecules. This strategy may protect infected cells from MHC class I-restricted cytotoxic T lymphocytes (CTL)-mediated lysis (40). HCMV genes that are involved in MHC class I downregulation are US2 (246), US3 (9, 247), US6 (10. 213) and US11 (545). MHC class I down-regulation was also observed in the murine system (83), in which gene products of $\mathrm{m} 152{ }^{(565)}, \mathrm{m06}{ }^{(424)}$ and $\mathrm{m04}{ }^{(264)}$ were shown to affect MHC class I complexes.

Although the downregulation of MHC class I expression may protect the cell from CTL restricted immune surveillance, absence of MHC class I molecules on the cellular membrane renders a cell susceptible for NK cell lysis, termed the 'missing self' hypothesis (316). HCMV encodes an MHC class I homolog, named UL18 (39). Similar genes, designated m144 and r144, were found in the genome of murine CMV (MCMV) ${ }^{(418)}$ and rat CMV (RCMV) (531), respectively. It was shown in the murine model, using an MCMV strain carrying a disruption in the $\mathrm{m} 144$ gene, that infection with this strain was associated with restricted replication in vivo (155). Further analysis revealed that NK cells were responsible for this attenuated phenotype (155). Inhibition of NK cell immune surveillance was also observed in vitro in both the human (428) and murine system (116, 282). However, it should be noted that expression of m144 only partially inhibited NK cell-mediated lysis (116, 282). Although UL18 gene product protected UL18-transfected cells in vitro from NK cell attack (428), Leong et al. did not find a positive correlation between UL18 and inhibition of NK cell immune surveillance using fibroblast infected with either WT HCMV or UL18 knockout virus (306).

Interestingly, m144 protected m144-transfected lymphoma cells from NK cellmediated rejection in vivo (116) and expression of $\mathrm{m} 144$ was associated with a reduction of leukocyte and NK cell recruitment and NK cell activation (116). These data are in concert with the evidence from the human and rat model, using virus mutants lacking the UL18 and r144 gene, respectively. The gene product of UL18 was found to associate with LIR-1, a member of the $\mathrm{lg}$ receptor superfamily, which is predominantly expressed on monocytes and $B$ lymphocytes and only on a subset of NK cells (114). Additionally, data from the rat 
model demonstrated that subcutaneous infection with an RCMV strain carrying a disruption of r144 gene (RCMV $\Delta$ r144) resulted in a significantly lower number of monocytes/macrophages compared to wild-type (wt) RCMV-infected animals. However, differences in the number of recruited NK cells between wt RCMV- and RCMV $\triangle$ r144infected tissue were not detected (43). These data suggest that the MHC class I homolog of $\mathrm{RCMV}$ is more likely to interfere with the innate immune response as a whole, rather than solely affect NK cell immune surveillance.

RCMV $\Delta$ r144 showed a similar phenotype in infected immunosuppressed rats as wt RCMV (43). Since it is thought that the MHC class I homolog may interfere with cells of the innate immune system, it is possible that potential differences between wt RCMV and RCMV-d-144 were concealed by the high level of irradiation-induced immunosuppression. We therefore employed a rat model with a natural, but still immature unaffected immune system. In this model, neonatal rats were infected with either wt RCMV or RCMV $\Delta$ r144. Rats were sacrificed at either 3,5 or 21 days p.i. and the amount of infectious virus and the presence of viral DNA was determined in various organs. In addition, the type and number of infiltrating leukocytes was determined by using immunohistochemistry.

Table 1.

The presence of infectious virus in wt-RCMV- or RCMVAr144-infected neonatal rats

\begin{tabular}{lcccc} 
& \multicolumn{2}{c}{ Spleen } & \multicolumn{2}{c}{ Liver } \\
\cline { 2 - 5 } & wt RCMV & RCMV $\Delta$ r144 & wt RCMV & RCMV $\Delta r 144$ \\
\hline $3^{0}$ & $170 \pm 86^{\circ}$ & $3 \pm 3^{*}$ & $0 \pm 0$ & $3 \pm 3$ \\
5 & $30 \pm 6$ & $10 \pm 7$ & $0 \pm 0$ & $7 \pm 7$ \\
21 & $0 \pm 0$ & $0 \pm 0$ & $0 \pm 0$ & $0 \pm 0$
\end{tabular}

\begin{tabular}{lcccc} 
& \multicolumn{2}{c}{ Lung } & \multicolumn{2}{c}{ Salivary gland } \\
\cline { 2 - 5 } & $w t R C M V$ & $R C M V \Delta r 144$ & $w t R C M V$ & $R C M V \Delta r 144$ \\
\hline $3^{\circ}$ & $0 \pm 0$ & $3 \pm 3$ & $0 \pm 0$ & $0 \pm 0$ \\
5 & $0 \pm 0$ & $3 \pm 3$ & $0 \pm 0$ & $0 \pm 0$ \\
21 & $0 \pm 0$ & $0 \pm 0$ & $45000 \pm 3200$ & $12500 \pm 740^{*}$
\end{tabular}

a Data is expressed as PFU/g tissue an is presented as mean \pm SEM.

b Days p.i.

- Significant when compared to wt RCMV $(p<0.05)$. 


\section{Materials and methods}

Virus

RCMV (Maastricht strain) was derived from salivary gland homogenates from immunosuppressed RCMV-infected rats as described previously (78). Prior to injection (see below), the salivary gland homogenate underwent several in vitro passages. RCMV $\Delta r 144$ was previously generated in our laboratory ${ }^{(43)}$ and was propagated in similar fashion as wt RCMV. Mock infection consisted of supernatant from uninfected rat embryonal fibroblasts (REF).

\section{Animals}

Inbred specific-pathogen-free (SPF) male and female Lewis rats were used at the age of 3 days. The animals were purchased from the Department of Experimental Animal Service at the University of Maastricht, Maastricht, The Netherlands. Animals were nourished by their mother until they reached the age of 21 days. Thereafter, the rats were fed normal rat chow and tap water ad libitum.

\section{Experimental design}

Rats were injected intraperitoneally (i.p.) with $200 \mu$ of REF supernatant, WT-RCMV or RCMV $\Delta$ r144. Viruses were administered at a dose of $3 \times 10^{4}$ PFU. Animals ( $n=5$ per group) were subsequently sacrificed at either 3,5 or 21 days post infection. At the time of sacrifice, spleen, liver, lung, salivary gland, kidney, hart, pancreas, thymus and bone marrow were harvested for the detection of infectious virus and viral DNA by plaque assay and PCR, respectively. The presence of inflammatory cells (NK cells, monocytes/macrophages and T cells) was assessed by immunohistochemistry.

\section{Plaque titration assay}

The titer of infectious virus in organs was determined by plaque assay using REF, as was described before (78). In short, the solid organs were homogenised and suspended in minimal essential medium (MEM) containing $2 \%$ fetal calf serum (FCS). Tenfold dilutions of $10 \%$ homogenates $(w / v)$ were inoculated on confluent REF monolayers. Bone marrow was extracted from both femurs and was dissolved in PBS and stored at $-70^{\circ} \mathrm{C}$. Before incubation on confluent REF, MEM containing $2 \%$ FCS was added to the bone marrow homogenate. A minimum of $1 \times 10^{6}$ cells was added to each well. All plaque assays were performed in triplicate. After an incubation period of 7 days, under $0.25 \%$ agarose, the number of plaques was determined microscopically after fixation and methylene blue staining. 
Organ samples were minced mechanically and DNA was isolated by using the Wizard $\AA$ Genomic DNA Purification Kit (Promega, Madison, WI, USA). During the isolation procedure, organ homogenates were incubated with proteinase $\mathrm{K}(60 \mathrm{mg} / 600 \mu \mathrm{l})$ at $60^{\circ} \mathrm{C}$ for 60-90 minutes. Viral DNA was detected by using a sensitive, single-tube, nested PCR which amplifies a part of the major immediate-early region of the RCMV genome as described previously (43). The PCR mixtures, with a total volume of $50 \mu \mathrm{l}$, contained $1 \mu \mathrm{g}$ of target DNA, $0.05 \mu \mathrm{M}$ of primers RIE3F and RIE3R2, $1 \mu \mathrm{M}$ of each primer RIE4F and RIE4R, $100 \mu \mathrm{M}$ of each dNTP, $1.25 \mathrm{U}$ of HotStar Taq DNA polymerase, HotStar Taq DNA polymerase buffer (Qiagen, Leusden, The Netherlands) and $15 \mathrm{mM} \mathrm{MgCl} 2$. Amplification was performed with a GeneAmp PCR System 9600 thermal cycler (Perkin-Elmer, Nieuwerkerk aan de IJssel, The Netherlands). PCR products were visualized by ethidium bromide-staining after gelelectrophoresis. To determine the relative concentration of RCMV DNA, the samples tested in serial 10-fold dilutions of target DNA. In addition, samples were tested with or without spike (10 copies of viral DNA).

\section{Immunohistochemistry}

Tissue samples were snap frozen and mounted in Tissue-Tec (Sakuro Finetek Europe BV, Zoeterwoude, The Netherlands). Immunohistochemistry was performed using 4- $\mu \mathrm{m}$ tissue sections. Monoclonals (MoAbs) ED-1, W3-13 and 3.2.3. were used for the detection of monocytes/macrophages (133), T-cells (71) and NK cells (91), respectively. Positive cells were visualized using HRP-labeled rabbit anti-mouse immunoglobulins (P260, Dako A/S, Glostrup, Denmark) and diaminobenzidine (DAB) substrate. The sections were counter-stained with hematoxilin and eosin. For the assessment of inflammation, the amount of inflammatory cells was counted in 5 independent high power fields (HPF, 400x) were counted and expressed as mean + SEM.

\section{Statistics}

For statistical analysis, the non-parametric Mann Withney $U$ test was used. Differences associated with $p<0.05$ were considered statistically significant. Data were presented as mean \pm SEM. 

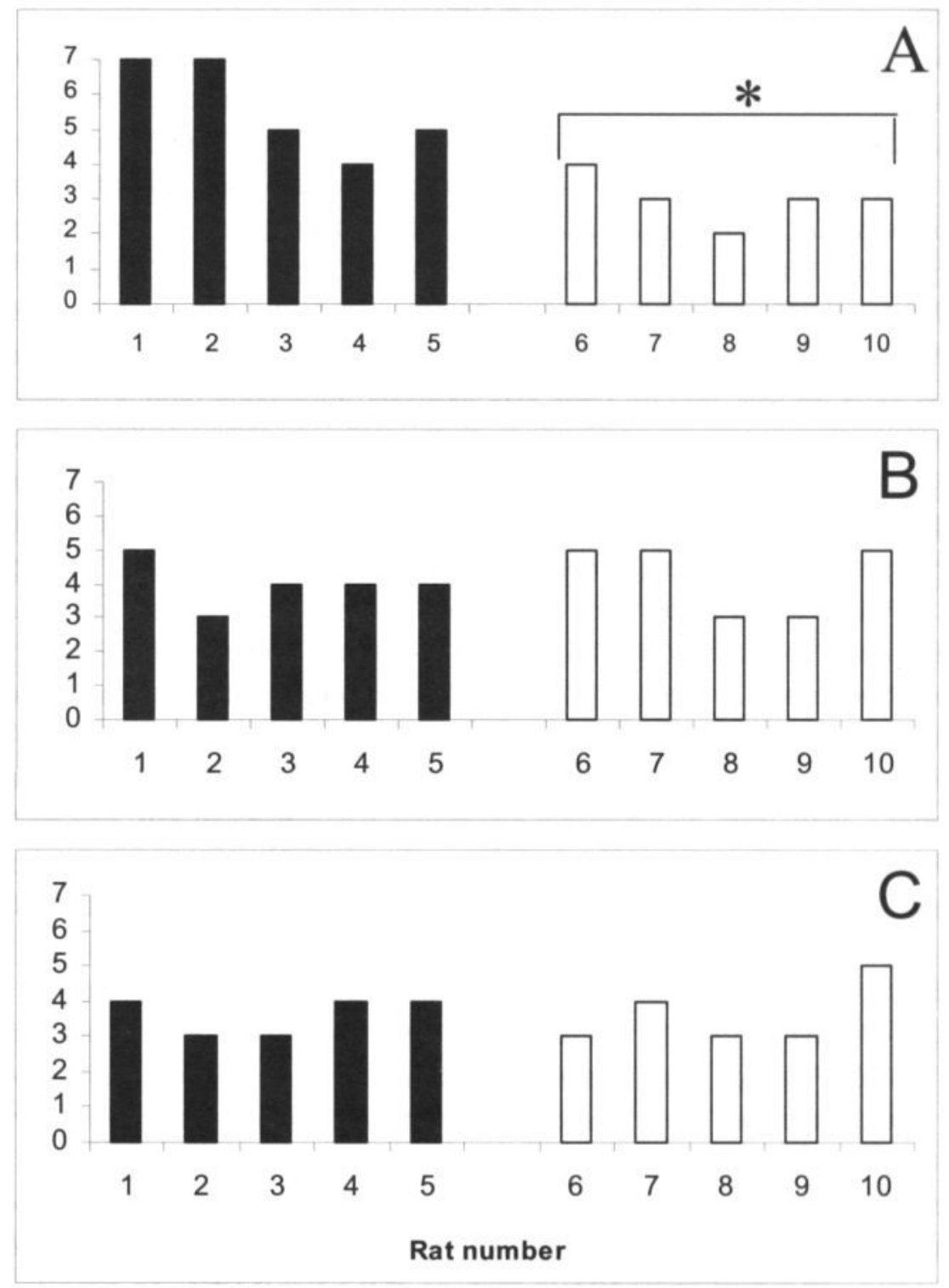

Figure 1. The relative concentration of viral DNA in organs from RCMV $\triangle$ r144- and wt RCMV-infected rats. The relative DNA concetration was determined in spleen (A), liver (B) and lung (C) at 3 days p.i, using 10 fold serial dilutions of target DNA. Each bar indicates the highest dilution of target DNA that was still found to be PCR-positive. Black bars represent wt-RCMV-infevcted rats whereas white bars represent RCMV $\Delta \mathrm{r} 144$-infected animals. ${ }^{*}=$ significantly different when compared to wt RCMV $(p<0.05)$. 


\section{Results}

RCMVAr144 shows restricted replication in salivary glands and spleen of neonatal rats

It was shown previously, that there are no significant differences in tissue distribution and virus titers between wt RCMV- and RCMV $\triangle$ r144-infected immunosuppressed adult rats ${ }^{(43)}$. Since it is thought that the $\mathrm{r} 144$ gene might play a role in virus-induced evasion of immune surveillance, it is possible that the high level of irradiation-induced immunosuppression was responsible for those results. In order to study the role of r144 in the pathogenesis of RCMV infection in rats with a normal, unaffected immune system, neonatal rats were challenged with either wt RCMV or RCMV $\triangle$ r144 and the presence of infectious virus as well as viral DNA was assessed in various organs at different time points p.i.

As expected, infectious virus could be demonstrated in spleen samples of wt RCMVinfected neonatal animals at 3 and 5 days p.i. (table 1). The amount of infectious virus declined significantly in this organ from $170 \pm 86 \mathrm{PFU} / \mathrm{g}$ at day 3 to $30 \pm 6 \mathrm{PFU} / \mathrm{g}$ at day 5 and $0 \pm 0 \mathrm{PFU} / \mathrm{g}$ at day 21 p.i. Interestingly, at day 3 p.i., the amount of infectious virus in the spleen was significantly lower in RCMV $\Delta$ r144-infected animals ( $3 \pm 3 \mathrm{PFU} / \mathrm{g}$ ) than in wt RCMV-infected rats $(170 \pm 86 \mathrm{PFU} / \mathrm{g})$. Significant differences in virus titer between both experimental groups were only found in spleens harvested at this time point. Very low virus titers were found in liver and lung of challenged animals at 3, 5 and 21 days p.i. In these organs, there were no significant differences between wt RCMV- and RCMV $\triangle \mathrm{r} 144$ infected rats in the amount of infectious virus. In accordance with previous studies $75,77$. 267), infectious virus could not be detected in the salivary gland at 3 and 5 days p.i.

At day 21 p.i., infectious virus was detected exclusively in the salivary glands of infected rats. Interestingly, the amount of infectious virus was significantly lower in RCMV $\Delta$ r144-infected rats $(12,500 \pm 740 \mathrm{PFU} / \mathrm{g})$ than in wt RCMV-infected animals $(45,000 \pm 3,200 \mathrm{PFU} / \mathrm{g}$ ). Infectious virus (wt RCMV and RCMV $\Delta \mathrm{r} 144$ ) was not detected in kidney, heart, pancreas, thymus and bone marrow samples at any of the time points measured (data not shown).

To further investigate the viral load in organs of wt RCMV- and RCMV $\triangle$ r144-infected rats, the relative concentration of viral DNA was assessed by a nested PCR assay (44), which was carried out on serial 10-fold dilutions of the target DNA. As expected, both wt RCMV and RCMV $\triangle$ r144 DNA could be detected in internal organs, such as spleen, liver and lung samples of all infected rats at 3 days p.i. However, at his time point, the concentration of viral DNA in the spleen was significantly lower in RCMVAr144-infected rats than in wt RCMV-infected animals (fig. 1a). In contrast, differences in viral DNA load between both experimental groups were not observed in liver and lung (fig. $1 \mathrm{~b}$ and $1 \mathrm{c}$ ). 
Table 2.

Presence of inflammatory cells ${ }^{a}$ in organs of wt RCMV- or RCMVAr144-infected neonatal rats.

\begin{tabular}{|c|c|c|c|c|c|c|c|}
\hline \multirow[t]{2}{*}{ Organ } & \multirow[t]{2}{*}{ Virus used } & \multicolumn{3}{|c|}{ Monocytes/macrophages } & \multicolumn{3}{|c|}{ T lymphocytes } \\
\hline & & 3 & 5 & 21 & 3 & 5 & 21 \\
\hline Spleen & $\begin{array}{l}\text { wt RCMV } \\
\text { RCMV }{ }^{\circ} 144^{\mathrm{b}} \\
\text { Mock }^{\mathrm{c}}\end{array}$ & $\begin{array}{l}90 \pm 8 \\
60 \pm 8^{*} \\
55 \pm 2^{*}\end{array}$ & $\begin{array}{l}111 \pm 10 \\
110 \pm 9 \\
53 \pm 1^{*}\end{array}$ & $\begin{array}{c}95 \pm 13 \\
90 \pm 7 \\
56 \pm 4^{*}\end{array}$ & $\begin{array}{c}94 \pm 13 \\
93 \pm 6 \\
58 \pm 1^{*}\end{array}$ & $\begin{array}{c}111 \pm 7 \\
118 \pm 17 \\
60 \pm 1^{*}\end{array}$ & $\begin{array}{l}97 \pm 13 \\
88 \pm 11 \\
57 \pm 0^{*}\end{array}$ \\
\hline Liver & $\begin{array}{l}\text { wt RCMV } \\
\text { RCMV } \Delta r 144 \\
\text { Mock }\end{array}$ & $\begin{array}{l}58 \pm 3 \\
58 \pm 5 \\
26 \pm 6^{*}\end{array}$ & $\begin{array}{l}61 \pm 2 \\
60 \pm 8 \\
21 \pm 0^{*}\end{array}$ & $\begin{array}{l}34 \pm 4 \\
37 \pm 7 \\
8 \pm 3^{*}\end{array}$ & $\begin{array}{l}12 \pm 4 \\
11 \pm 2 \\
4 \pm 2^{*}\end{array}$ & $\begin{array}{l}10 \pm 2 \\
11 \pm 3 \\
2 \pm 0^{*}\end{array}$ & $\begin{array}{l}7 \pm 1 \\
7 \pm 2 \\
2 \pm 1^{*}\end{array}$ \\
\hline Lung & $\begin{array}{l}\text { wt RCMV } \\
\text { RCMV } \Delta r 144 \\
\text { Mock }\end{array}$ & $\begin{array}{l}28 \pm 2 \\
28 \pm 5 \\
11 \pm 1^{*}\end{array}$ & $\begin{array}{l}34 \pm 1 \\
30 \pm 2 \\
9 \pm 1^{*}\end{array}$ & $\begin{array}{c}35 \pm 2 \\
36 \pm 2 \\
10 \pm 3^{*}\end{array}$ & $\begin{array}{l}25 \pm 2 \\
25 \pm 1 \\
11 \pm 0^{*}\end{array}$ & $\begin{array}{l}26 \pm 3 \\
24 \pm 3 \\
17 \pm 4^{*}\end{array}$ & $\begin{array}{l}50 \pm 2 \\
55 \pm 1 \\
20 \pm 2^{*}\end{array}$ \\
\hline $\begin{array}{l}\text { Salivary } \\
\text { gland }\end{array}$ & $\begin{array}{l}\text { wt RCMV } \\
\text { RCMV } \Delta r 144 \\
\text { Mock }\end{array}$ & $\begin{array}{l}13 \pm 3 \\
13 \pm 2 \\
10 \pm 6\end{array}$ & $\begin{array}{l}15 \pm 1 \\
14 \pm 2 \\
4 \pm 1^{*}\end{array}$ & $\begin{array}{l}17 \pm 2 \\
15 \pm 3 \\
2 \pm 2^{*}\end{array}$ & $\begin{array}{l}1 \pm 0 \\
1 \pm 0 \\
1 \pm 0\end{array}$ & $\begin{array}{l}2 \pm 1 \\
1 \pm 1 \\
0 \pm 0\end{array}$ & $\begin{array}{l}3 \pm 1 \\
2 \pm 2 \\
0 \pm 0^{*}\end{array}$ \\
\hline
\end{tabular}

\begin{tabular}{llccc} 
Organ & Virus used & \multicolumn{3}{c}{ NK cells } \\
\cline { 3 - 5 } & & 3 & 5 & 21 \\
\hline Spleen & wt RCMV $^{\circ}$ & $17 \pm 1$ & $24 \pm 3$ & $29 \pm 4$ \\
& RCMV $\Delta r 144^{\mathrm{b}}$ & $12 \pm 0^{*}$ & $25 \pm 2$ & $24 \pm 8$ \\
& Mock $^{\mathrm{y}}$ & $7 \pm 0^{*}$ & $5 \pm 0^{*}$ & $11 \pm 1^{*}$ \\
Liver & wt RCMV & $11 \pm 4$ & $15 \pm 4$ & $1 \pm 0$ \\
& RCMV $\Delta \mathrm{r} 144$ & $9 \pm 3$ & $12 \pm 3$ & $2 \pm 0$ \\
& Mock & $2 \pm 1^{*}$ & $1 \pm 0^{*}$ & $0 \pm 0$ \\
\multirow{4}{*}{ Lung } & wt RCMV & $9 \pm 1$ & $14 \pm 1$ & $7 \pm 1$ \\
& RCMV $\Delta \mathrm{r} 144$ & $8 \pm 2$ & $13 \pm 1$ & $7 \pm 1$ \\
& Mock & $1 \pm 1^{*}$ & $1 \pm 1^{*}$ & $2 \pm 1^{*}$ \\
Salivary & wt RCMV & $1 \pm 0$ & $1 \pm 1$ & $4 \pm 0$ \\
gland & RCMV $\Delta \mathrm{r} 144$ & $1 \pm 0$ & $1 \pm 0$ & $4 \pm 1$ \\
& Mock & $0 \pm 0$ & $0 \pm 0$ & $0 \pm 0^{*}$
\end{tabular}

a The number of inflammatory cells was counted in 5 independent HPF fields. Data are expressed as mean \pm SEM.

b Rats were ip infected at a dose of $3 \times 10^{4} \mathrm{PFU}$

c Mock infection consisted of REF supernatant

- significantly different when compared to wt RCMV $(p<0.05)$ 
Taken together, these data show that in comparison with wt RCMV-infected rats, RCMV $\Delta$ r144-infected animals have a significantly lower viral load in spleen and salivary gland. These organs have previously been shown to be the most important target organs for RCMV replication (497).

The number of leukocytes in the spleen corresponds with the viral load in this organ

The RCMV r144-encoded MHC class I homolog may interfere with cells of the innate immune system in order to evade immune surveillance. To explore this possibility, we studied the number monocytes/macrophages, T lymphocytes and NK cells in spleen, liver, lung and salivary gland of wt RCMV- and RCMV $\triangle$ r144-infected rats (table 2). Similar to the situation in immunocompromised adult rats $(399,497)$, wt RCMV infection resulted in a significantly higher number of inflammatory cells in spleen, liver, lung and salivary gland than did mock infection (table 2). Interestingly, at 3 days p.i., significantly lower numbers of monocytes/macrophages and NK-cells were found in spleens of RCMVAr144-infected rats than in spleens of wt RCMV-infected animals. Thus, the number of monocytes/macrophages and NK-cells corresponds with the viral load in these organs. At 5 days p.i., we did not find significant differences between spleens of wt RCMV- and RCMV $\Delta$ r144-infected rats in the number of recruited monocytes/macrophages, $T$ cells and NK-cells. In addition, the number of inflammatory cells present in liver, lung and salivary gland does not differ between both experimental groups at all three time points measured (table 2).

In conclusion, these data indicate that the number of monocytes/macrophages and NK cells present in the spleen of infected neonatal rats is directly proportional to the amount of infectious virus in this organ.

\section{Discussion}

Experimental infection of immunocompetent rats with RCMV is predominantly characterised by replication of virus in salivary gland cells $(75,77)$. Dissemination of high levels of virus is more widespread in animals receiving total body-irradiation (TBI) prior to injection of virus and involves spleen, liver, lung and salivary gland (497). In addition, RCMV disease can only be observed in lethally infected rats with irradiation-induced immunosuppression (268). The administration of a single dose of 5 Gy TBI as immune suppressive regimen affects the number of granulocytes, NK cells, monocytes, B cells and $\mathrm{T}(\mathrm{CD} 4+$ and $\mathrm{CD} 8+)$ cells in peripheral blood of irradiated animals significantly and leads to a transient state of severe immunosuppression (525). Peripheral white blood cells are suppressed during the acute phase of infection and the number of cells return to control levels at approximately 3-4 weeks after irradiation (525). 
We previously demonstrated that the r144 gene is not essential for virus replication shortly after infection, nor for long-term infection in immunocompromised rats (43). Since it is thought that the r144-encoded MHC class I homolog may interfere with the function of the innate immune system, the immunosuppressive regimen that we previously used may mask potential differences in replication between wt RCMV and RCMV $\triangle$ r144. We therefore employed an alternative model in which neonatal rats were used with a natural, unaffected immune system and infected them with either wt RCMV or RCMV $\triangle$ r144. In this model, we found that: (i) the deletion mutant virus, RCMV $\Delta$ r144, replicated to significantly lower virus titers than wt RCMV in spleens and salivary glands at 3 days and 21 days p.i., respectively and (ii) the number of monocytes/macrophages and NK cells in the spleen was significantly lower in RCMV $\triangle$ r144-infected rats than the wt RCMV-infected animals at 3 days p.i.

RCMV $\Delta$ r144 has previously been shown to have similar replication characteristics as wt virus in vitro ${ }^{(43)}$. In addition, significant differences in virus titers between spleens of RCMV $\Delta$ r144- and wt RCMV infected immunosuppressed adult rats were not detected (43). These data suggest that RCMV $\triangle \mathrm{r} 144$ replicates as efficiently as wt RCMV in the absence of a fully functional immune system. In neonatal rats with a normal, unaffected immune system, the viral load in both salivary gland and spleen were significantly lower in RCMV $\Delta$ r144- than in wt RCMV-infected rats. This implies that differences in phenotype between both viruses in this model may be governed by the status of the immune system.

As described above, spleens of neonatal rats infected with RCMV $\Delta r 144$ harbored significantly lower numbers of monocytes/macrophages and NK cells than those infected with wt RCMV. In contrast, T lymphocytes were present in similar amounts in this organ in both experimental groups. This indicates that the r144 gene product may be involved in the interaction with cells of the innate immune system, rather than with cells of the cellmediated immune response. Furthermore, we previously demonstrated that subcutaneous infection of the rat hind paw with either wt RCMV of RCMV $\triangle$ r144 resulted in a significantly lower number of monocytes/macrophages in subcutaneously infected rat hind paws of RCMV $\Delta$ r144-infected rats than in those of wt RCMV-infected animals (43). In this model, the viral load in the rat hind paw was similar between both experimental groups (43). However, differences in the number of NK cells in the rat hind paw between wt RCMV- and RCMV $\Delta$ r144-infected animals could not be detected (43). Our data from the neonatal model and rat hind paw model are in agreement with previous findings from the MCMV/murine model (116). It was shown that the MCMV homolog of r144, m144, protects lymphoma cells from NK cell-mediated rejection in vivo by regulation of the total leukocyte- and NK cell recruitment and NK cell activation (116). Since cytokines are important for the recruitment of NK cells and other leukocytes $(381,443,473)$, it was hypothesized by Cretney et al., that interaction of the murine MHC class I homolog with mononuclear cells may interfere with 
the cytokine production of these cells, which eventually diminishes the innate immune response as a whole (116). Similarly, the MHC class I homolog encoded by HCMV, UL18, may be more important in affecting monocyte and dendritic cell function during CMV infection, rather than mediate NK cell immune surveillance (306).

As expected, the salivary gland harbored high amounts of infectious virus at 21 days p.i. Interestingly, the amount of infectious virus in this organ was significantly lower in RCMV $\Delta$ r144-infected rats than in wt RCMV-infected animals. In contrast to what was seen in the spleen, virus titers did not correlate with the numbers of infiltrating leukocytes in the salivary glands: similar numbers of these leukocytes were detected in the glands of wt RCMV- and RCMV $\triangle$ r144-infected animals. This apparent discrepancy can be explained by the observation that the inflammatory response in the salivary gland is restricted to the interlobular duct region, whereas the virus replicates in the striated duct cells of the intralobular duct (267).

The exact nature of the attenuation of RCMV $\Delta r 144$ as opposed to the wt virus in neonatal rats is still unknown. Also, it is not clear whether the attenuated growth of the recombinant virus in salivary glands and spleen is either specific for these organs or the result of a more 'general' inability of the virus to replicate efficiently. These and other questions on the role of r144 in the replication of RCMV will have to be addressed in future studies. 
$\sim$ Chapter $5 \sim$

Rat cytomegalovirus infection in kidney allograft recipients is

associated with increased expression of ICAM-1, VCAM-1 and their ligands

LFA-1 and VLA-4 in the graft. 


\section{Summary}

Cytomegalovirus (CMV) infection is suggested to be a risk factor for chronic rejection. We have recently shown that rat $\mathrm{CMV}$ (RCMV) increases the inflammatory response and accelerates chronic rejection in a model of rat kidney allograft (293). In this study, the early inflammatory response and time-related expression of intercellular adhesion molecule-1 (ICAM-1) and vascular cell adhesion molecule-1 (VCAM-1) and their ligands, leukocyte function antigen-1 (LFA-1) and very late antigen-4 (VLA-4), were assessed in kidney allografts of RCMV infected rats and compared to non-infected rats developing chronic rejection. Kidney transplantations were performed in a rat strain combination of DA $\left(R T 1^{2}\right)$ to $\mathrm{BN}\left(\mathrm{RT} 1^{\mathrm{n}}\right)$ receiving triple drug immunosuppression. One group of rats was infected with RCMV and the other group was left uninfected. The grafts were harvested at different time points after transplantation. The adhesion molecules, their ligands and activation markers, i.e. MHC class II antigen expression and interleukin-2-receptors (IL-2-R), were demonstrated by monoclonal antibodies and immunoperoxidase staining of frozen sections of the grafts. Graft histology was evaluated according to the Banff criteria. RCMV caused a significant, prolonged increase of VCAM-1 and ICAM-1 expression in the vascular endothelium compared to the non-infected grafts. Also, the number of cells expressing activation markers, LFA-1 and VLA-4 was significantly enhanced in these animals. Significantly enhanced histological changes indicative for chronic rejection were seen in the RCMV infected group. In conclusion, increased expression of ICAM-1 and VCAM-1 and increased numbers of inflammatory cells expressing their ligands in the CMV infected grafts, were associated with accelerated chronic allograft nephropathy. 


\section{Introduction}

The etiology of chronic rejection is multifactorial and several risk factors have been described (14,38, 209, 390), such as acute rejection episodes, ischemia/reperfusion injury and viral infections, especially cytomegalovirus (CMV) infections. CMV infection has previously been suggested to trigger acute rejection of kidney allografts in clinical transplantation (407. 423, 535). Although CMV has been shown to be associated with chronic rejection of heart (193. 277. 339), lung (281) and liver transplants $(20,292,379)$, little is known about CMV and chronic rejection in renal transplantation.

Histological findings of chronic rejection are well defined and are characterised by focal interstitial lymphocytic inflammation and fibrosis, glomerular mesangial matrix increase and sclerosis, vascular intimal proliferation and tubular atrophy $(391,392,516)$. These variables can be summarised in the chronic allograft damage index (CADI) which can be used as a predictive parameter for chronic rejection $(233,234)$.

The inflammatory response and T-cell activation seem to be necessary for the development of chronic rejection (209, 390). Vascular adhesion molecules are important in the early phase of the alloresponse, cell-cell interactions, T-cell activation and extravasation of inflammatory cells into the organ $(67,172,220,534)$. Firm adhesion of leukocytes to the endothelial cells is established by the binding of intercellular adhesion molecule-1 (ICAM1) and vascular cell adhesion molecule-1 (VCAM-1) to the integrin molecules expressed on leukocytes, such as lymphocyte function associated antigen-1 (LFA-1) and very late antigen-4 (VLA-4) $(324,405,494)$. The induction of adhesion molecules is mediated by cytokines, such as IFN-gamma, TNF-alpha and IL-1, produced during the inflammatory process of the alloresponse.

We have recently developed an experimental model, in which rat renal allografts after an early inflammatory episode, develop chronic rejection under triple drug therapy of steroids, azathioprine and cyclosporin, within 40-60 days after transplantation (483). We have also studied the early phase of the process in detail and recorded that the peak of inflammation, seen 5-10 days after transplantation, is associated with lymphoid activation and induction of vascular adhesion molecules, such as ICAM-1 and VCAM-1. However, the early expression of adhesion molecules vanishes together with immune activation associated with acute rejection, while the changes associated with chronic rejection increase (259).

In this experimental model, we have also used the appropriate rat virus (RCMV) in order to study the processes involved in chronic rejection complicated by CMV infection (294, 483). RCMV increases and prolongs the early inflammatory response up to 20 days and accelerates the development of chronic rejection. In this model, RCMV infects the graft and RCMV specific antigens were found in the endothelial cells of the small vessels as well as in the smooth muscle cells of the large arteries (294). The CMV infected rats 
developed an end-stage chronic rejection with graft vasculopathy and remarkable fibrosis within 20 days after transplantation (294). In this study, we have investigated in detail the impact of RCMV on the early phase of the immunological process, at the level of lymphoid activation and induction of vascular adhesion molecules.

\section{Materials and methods}

\section{Animals}

Male inbred Dark Agouti (DA; RT1 $\left.{ }^{\mathrm{a}}\right)$ and Brown Norway $\left(\mathrm{BN} ; \mathrm{RT} 1^{\mathrm{n}}\right)$ rats weighing 200$300 \mathrm{~g}$ were used in the experiments. The rats were housed according to the international principles of laboratory animal care and were fed normal rat chow and tap water ad libitum. The experiments were approved by the Committee for Experimental Research of Helsinki University Central Hospital and the regional authorities.

\section{Transplantation and immunosuppression}

Transplantations were performed from DA to BN as previously described $(300,483)$. In short, the rats were anaesthetised with midazolan (Dormicum ${ }^{R}$ ) and fentanyl-fluanisone $\left(\right.$ Hypnorm $\left.^{\mathrm{R}}\right)$. After dissection of the kidney to be transplanted, the organ was flushed with ice cold Euro-Collins solution, containing $500 \mathrm{IU}$ of heparin/ml. The renal artery, renal vein and proximal ureter of the kidney from the donor were anastomosed to the aorta, inferior vena cava and distal ureter of the recipient, respectively. Total ischemia time was $30 \pm 10$ $\mathrm{min}$. The recipient's native right kidney was removed after implantation of the graft. The left native kidney of the recipient was left intact and used as an internal control. Immunosuppression consisted of methylprednisolone (2 mg/kg), azathioprine $(2 \mathrm{mg} / \mathrm{kg}$ ) and cyclosporin ( $5 \mathrm{mg} / \mathrm{kg}$ ), which was administered subcutaneously on a daily basis during the experiment. Autograft transplantations with the same cold and warm ischemia times were performed, also triple drug immunosuppression was given and the autografts were used as control. Rats receiving an autograft were left uninfected.

\section{Viral infection}

One group of the allografted animals was infected with RCMV (Maastricht strain). The RCMV inoculum was derived from homogenised salivary glands and was injected 1 day post transplantation, intraperitoneally at a dose of $1 \times 10^{5}$ plaque-forming units. The characteristics of RCMV infection and the method of viral inoculation, have been described in detail previously $(75,77)$. The infection was confirmed by viral cultures from the grafts on day 7 after inoculation as described (294). The infectivity of rat kidneys and the appearance of viral antigens in the grafts have been described in detail in our previous article (294). 


\section{Histology}

The grafts were harvested at $3,5,7,10,20$ and 30 days after transplantation and fixed in $10 \%$ buffered formalin. Each experimental group contained 4 animals at each timepoint. Specimens were paraffin embedded, cut $(4 \mu \mathrm{m})$ and stained with haematoxylineosin and Masson's trichrome. The Banff criteria (480), without grading, were used to assess the graft histology. The numerical chronic allograft damage index (CADI) was used to quantify the chronic alterations characteristic of chronic rejection, as described previously $(233,234)$ : focal interstitial lymphocytic inflammation, fibrosis, glomerular sclerosis, mesangial matrix increase, vascular intimal proliferation and tubular necrosis. The graft histology of this model with and without CMV is described in detail in our previous articles (294, 483).

\section{Immunohistochemistry}

The expression of vascular adhesion molecules and the number of inflammatory cells expressing their ligands and activation markers were immunohistochemically evaluated from frozen sections $(3-5 \mu \mathrm{m})$ of the kidneys. After fixation in cold acetone, an indirect immunoperoxidase technique and monoclonal antibodies against ICAM-1 (R\&D Systems Europe LTD, Abingdon, UK), VCAM-1 (a gift from Dr. R.Lobb, Biogen, Cambridge, MA), LFA-1 (R\&D Systems), VLA-4 (Pharmingen, San Diego, CA) , IL-2R (Sera-lab, Sussex, UK), and rat MHC class II (Sera-Lab, Sussex, UK) were used. The frozen sections were first incubated with the monoclonal antibodies. A peroxidase conjugated rabbit-antimouse (Dako, Glostrup, Denmark) and a peroxidase-conjugated goat anti-rabbit (Zymed Laboratories, San Francisco, CA) were used as second and third antibodies. The reaction was revealed by 3-amino-9-ethyl carbazole solution containing hydrogen peroxide. Mayer's hemalum was used for counterstaining. The expression of vascular adhesion molecules in kidney samples was assessed and the intensity of expression was graded from 1 to 3 . The numbers of infiltrating inflammatory cells positive for the ligand molecules, IL-2-R and class II were counted separately in different high power visual fields (magnification $x 400$ ). Three randomly chosen high power fields were analysed in each kidney. All analyses were carried out blindly by two or three observers.

\section{Statistics}

Intensity of adhesion molecule expression (ICAM-1 and VCAM-1 were assessed on different tissue sections) was expressed as median values. The number of cells (each marker was assessed on different tissue sections) which showed positive for LFA-1, VLA$4, \mathrm{IL}-2 \mathrm{R}$ and MHC class II were expressed as mean \pm SEM. For statistical analysis, the student $t$-test was used and $p$-values $<0.05$ were considered to be significant. 

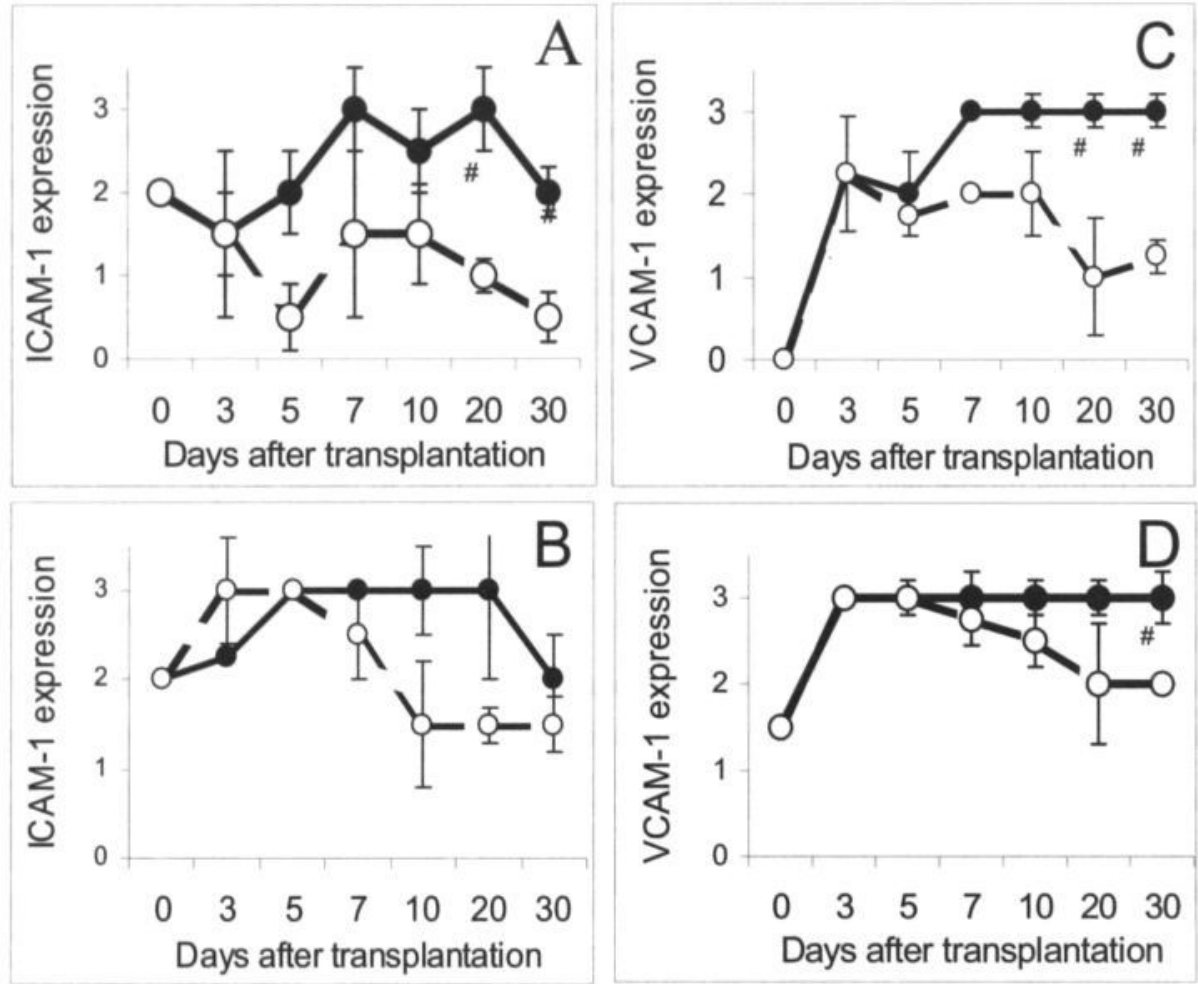

Figure 1. Time related follow-up of the intensity of ICAM-1 and VCAM-1 expression, graded from 1 to 3 (median), on endothelia of renal allografts during the development of chronic rejection in RCMV infected and non-infected animals. A) ICAM-1 expression on endothelium of larger vessels. B) ICAM-1 expression on capillary endothelium. C) VCAM-1 expression on endothelium of larger vessels. D) VCAM-1 expression on capillary endothelium. Straight lines = RCMV infected rats; dashed lines = non-infected rats; \# = significantly different when comparted to controls, $p<0.05$.

\section{Results}

Expression of vascular adhesion molecules and their ligands in the graft in relation to the development of chronic rejection

In the phase shortly after transplantation, at day 7, grafts showed signs of vascular damage and strong interstitial inflammation, which consisted predominantly of lymphocytes. As was already described in our previous study (294), in non-infected grafts, the early inflammation was seen 5-10 days after transplantation and CMV prolonged the inflammation so that it was seen from day 5 onwards, until the grafts ended-up with chronic rejection at day 20 after transplantation. The grafts from non-infected rats showed less intense inflammation and only mild vascular changes at the early post transplantation course. The CMV infected grafts developed chronic rejection within 20 days after 
transplantation, according to the histological criteria of chronic rejection. In grafts harvested from RCMV infected animals, characteristic findings of chronic rejection were recorded, as described previously with significantly higher CADI values also than in the non-infected grafts $(9.0 \pm 0.5$ versus $5.8 \pm 1.7, p<0.05)$. The histological findings of this experiment were described in detail in our previous article ${ }^{(294)}$ and are not repeated here.

In the RCMV infected group, expression of ICAM-1 and VCAM-1 of endothelium in the larger vessels, increased to a maximum at 7 days and virtually remained at this level during the follow-up until 30 days post transplantation (Figure 1). In the non-infected grafts, induction of the adhesion molecules was also seen shortly after transplantation, but the expression decreased during the course of the development of chronic changes. The prolonged expression of both ICAM-1 and VCAM-1 was significant $(p<0.05)$ in the vascular endothelium of larger vessels compared with the non-infected grafts (Figure 1). In the capillary endothelium, a significant $(p<0.05)$, prolonged VCAM-1 expression was also observed and ICAM-1 showed the same tendency, although the difference was not statistically significant. Induction of tubular expression of ICAM-1 and VCAM-1 was also recorded with the expression of ICAM-1 (median 2.0) being more intense than that of VCAM-1 (median 0.5), but no statistically significant difference was found between the RCMV infected and the non-infected animals (data not shown).

Expression of LFA-1 and VLA-4, the ligands for ICAM-1 and VCAM-1 respectively, on infiltrating lymphocytes was assessed. LFA-1 and VLA-4 positive cells were abundantly present in non-infected animals at 3-4 days after transplantation and the number of these cells decreased steadily during the development of histological changes indicative for chronic rejection. However, RCMV infection resulted in a significant increase in the number of LFA-1 positive and VLA-4 positive cells present in the allograft when compared to non-infected animals. (Figure 2).

\section{Expression of lymphoid activation markers IL-2R and MHC class II}

In the inflammatory infiltrate, the number of IL-2R positive cells in allografts harvested from RCMV infected animals was significantly increased during the early phase of the process, compared to the non-infected rats. Approximately the same pattern of cellular expression was also recorded for MHC class II. (Figure 3). 

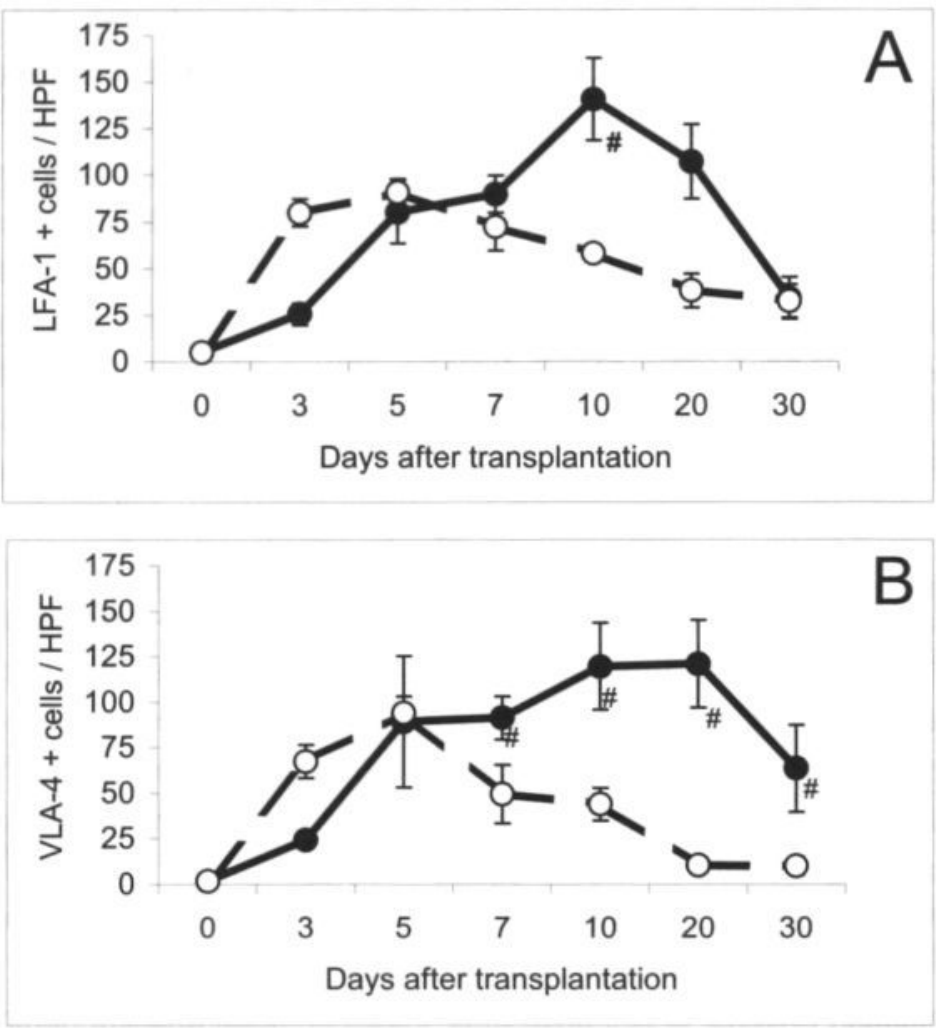

Figure 2. The time related follow-up of the number of allograft infiltrating inflammatory cells counted per high power visual field (mean \pm SEM) expressing LFA-1 (A) and VLA-4 (B) during the development of chronic rejection with and without concomitant RCMV infection. Straight lines = RCMV infected rats; dashed lines = non-infected rats; \# = significantly different when comparted to controls, $\mathrm{p}<0.05$.

\section{Controls: Autografts and own kidneys}

In autografted kidneys, only a slight temporal expression of ICAM-1 and VCAM-1 was recorded, located in the vascular and capillary endothelium at 5 days after transplantation (not shown). Thereafter the autografts showed a similar staining pattern as control kidneys i.e. the own left native kidney. Autografted kidneys were characterised by an absence of inflammatory cells and the number of cells expressing LFA-1, VLA-4 and IL-2R was similar to that of the normal own kidneys (not shown). During follow up, kidneys did not show any signs of chronic rejection (CADI < 2.0). Minor surgical inflicted injuries were seen in the tubuli and vascular structures immediately after transplantation, at days $3-5$, but the only long-term histological finding of the autografts was slight diffuse fibrosis of the kidney in 
two animals at day 20 and in one animal at day 30 after transplantation. No significant vascular, tubular or glomerular changes were recorded at days 20 and 30 .

The own kidneys of the CMV infected rats showed the same expression pattern as the own control kidneys of the non-infected animals at each time point up to 30 days post transplantation. No induction of adhesion molecules was recorded and no inflammatory cell infiltration with the expression of their ligands were seen in the own kidneys of CMVinfected or non-infected animals. No histological changes were recorded in the control own kidneys of both animal groups $(\mathrm{CADI}=0)$.

\section{Discussion}

CMV infection increased the inflammatory response in our rat renal allograft model and accelerated the development of chronic rejection, as was reported in our previous series (294). As was also described previously, in non-infected grafts there was an early inflammatory response between 5-10 days after transplantation, but in the CMV infected grafts an increased inflammation was seen (294). CMV also prolonged the inflammation so that it was still prominent at day 20 after transplantation, when the histological criteria of chronic rejection were fulfilled (294). Enhanced chronic rejection with vascular changes and prominent fibrosis and also with high CADI values, on day 20 in the CMV infected animals, corresponded with the findings of non-infected grafts in our chronic rejection model of days 40-60 post transplantation $(259,294)$.

In the present study, we have further demonstrated that CMV infection also resulted in a significant and prolonged increase of VCAM-1 and ICAM-1 expression on the vascular endothelium in the renal allografts. The difference between the groups was clear from day 5 onward, but the statistical significance was reached later together with the histological criteria of chronic rejection. In addition, the number of infiltrating cells expressing the ligand molecules LFA-1 and VLA-4, as well as activation markers, was significantly increased in these animals corresponding to the prolonged inflammatory response. Autografted kidneys harvested from non-infected rats were used as controls. Autografts did not show a significant induction of adhesion molecules and no inflammatory response was seen eithe. In histology, only slight fibrosis was recorded at the end of the follow-up. The native own kidneys of CMV-infected and non-infected animals demonstrated the same expression pattern and no inflammatory infiltrate or histological changes were recorded.

The induction of these adhesion molecules is mediated by cytokines, such as IL-1, TNFalpha and IFN-gamma, which are produced by activated lymphocytes during the alloresponse. The role of adhesion molecules and cytokines in the induction of the process of chronic rejection has been shown in rat renal allograft models $(205,259)$. 

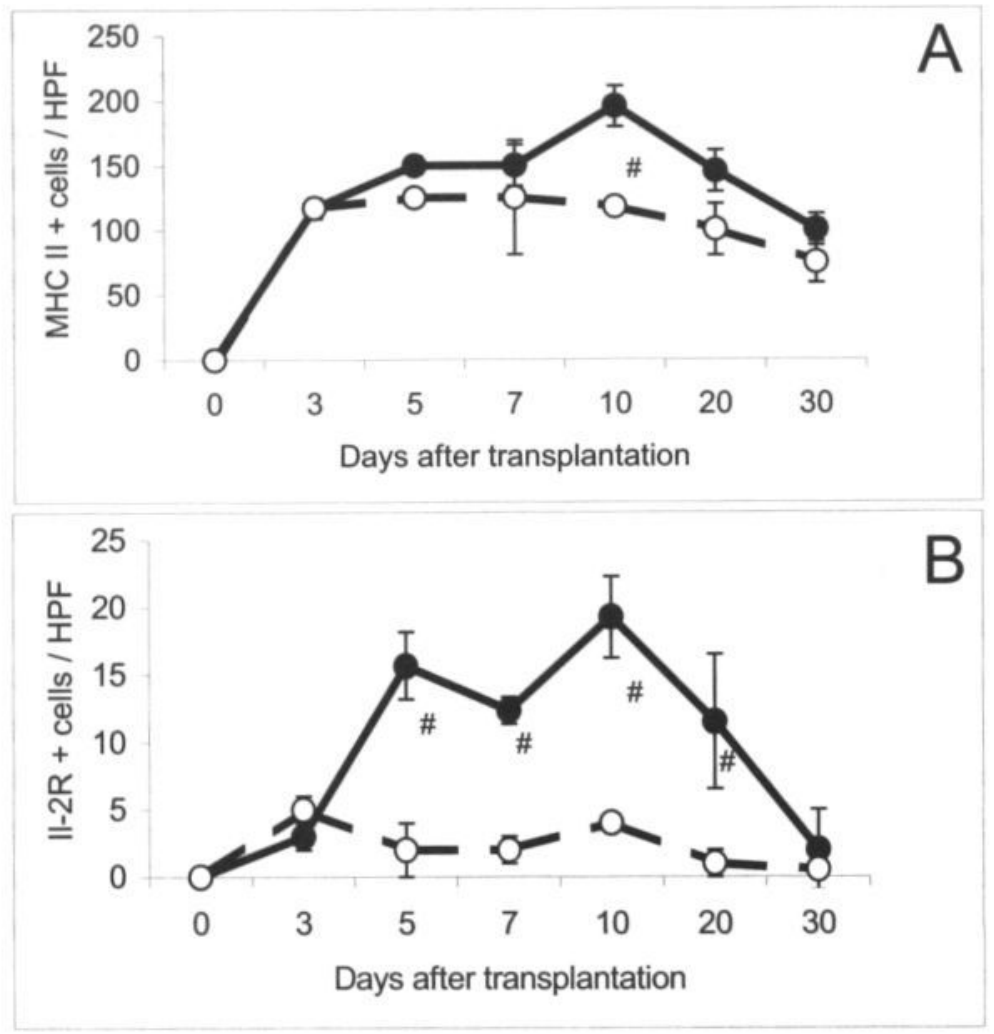

Figure 3. Time related follow-up on the number of allograft infiltrating inflammatory cells counted per high power visual field (mean \pm SEM) expressing activation markers MHC class II (A) and IL-2R (B) molecules during the development of chronic rejection in RCMV infected and non-infected rats. Straight lines = RCMV infected rats; dashed lines = non-infected rats; \# = significantly different when comparted to controls, $p<0.05$.

On the other hand, it has been shown in vitro that $\mathrm{T}$ lymphocytes which are activated by $\mathrm{CMV}$ infected endothelial cells, may trigger cytokine mediated expression of adhesion molecules on non-infected endothelial cells (541). In addition, it has been demonstrated that one week after inoculation, RCMV is predominantly found in capillary endothelium of allografted kidneys, also in the smooth muscle cells of the arterial wall, but not in the endothelium of larger arteries (294). So this might also explain why the difference in ICAM-1 and VCAM-1 expression between RCMV infected rats and non-infected animals was more noticeable on larger vessels than on capillary endothelium in our assessment. CMV has also been found to upregulate IL-1-beta gene expression, which may lead to production of $\mathrm{IL}-1$ by mononuclear cells ${ }^{(235)}$. CMV induces the production of TNF-alpha in monocytes and macrophages as well (472). The same kind of two-way activation also regulates the 
CMV-TNF-alpha co-reactivity. However, the CMV induced T-cell activation, production of cytokines and the increase of adhesion molecule expression may thus be involved in the alloresponse and trigger the process of chronic rejection. In the own native kidneys, no changes were demonstrated, which shows the importance of the immunological triggering in the graft. Thus, the alloresponse seems to be necessary for the CMV associated changes, as was already recorded in our previous study on rat aorta allografts (304).

The involvement of inflammation, adhesion molecules and cytokines has been studied in vitro and in vivo in animal models. In experimental models of lung (506) and liver (329) allografts, CMV infection has been reported to induce lymphocyte dominated inflammation, T-cell activation and adhesion molecules in the graft. In another experimental model of renal allografts, in which the animals underwent acute rejection episodes and received cyclosporin only as immunosuppressive treatment, increased ICAM-1 expression was recorded in the graft at the end-stage of the process in the CMV infected animals (557), Our sequential follow-up of the development of chronic rejection demonstrated that the involvement of CMV infection occurs already at the induction phase of the immunological cascade in the graft, at the level of T-cell activation and lymphocyte extravasation. CMV associated peripheral $\mathrm{T}$ cell activation in long term renal transplant patients has been described (262). However, these immunological events should also be studied in the clinical series of human kidney allografts complicated with CMV infection. Although the role of adhesion molecules in chronic rejection has been studied in human renal allografts (184, 481, 533), no clinical report exists describing the impact of $\mathrm{CMV}$ on these findings.

In conclusion, prolonged, increased expression of adhesion molecules ICAM-1 and VCAM-1 on the vascular endothelium and increased numbers of cells positive for their ligand molecules LFA-1 and VLA-4 in kidney allografts of CMV infected rats, was linked with the acceleration of chronic nephropathy in this model. The CMV associated, prolonged inflammation correlated with the increased number of infiltrating cells expressing activation markers. These findings indicate that the prolonged activation of the immunological cascade in the CMV infected grafts was associated with accelerated chronic nephropathy. 


\section{Acknowledgements}

The authors wish to thank Dr. Roy Lobb and Biogen Inc. for providing the anti-rat VCAM-1 antibody. The authors also thank Mr. Stephen Venn for correcting the English text and Kari Savelius for animal care.

This work was supported by grants from the Sigrid Juselius Foundation, Helsinki University Central Hospital Research Funds (EVO), the Finnish Academy of Science and the NWOgebied Medische Wetenschappen, the Netherlands. 
$\sim$ Chapter 6

Effective treatment of experimental CMV-induced encephalo-meningitis in immunocompromised rats with HPMPC. 


\section{Summary}

Cytomegalovirus (CMV) -induced encephalomeningitis is a dramatic complication in patients with the acquired immunodeficiency syndrome (AIDS) and treatment of this infection remains a major clinical problem. In order to study the pathogenesis and treatment of CMV-induced encephalomeningitis, we experimentally induced intracranial rat-CMV (RCMV) infection in rats that were immunosuppressed by total body $\mathrm{X}$-irradiation. CMV infection was monitored by viral plaque assay for estimation of the viral load. CMV-induced pathology, the presence of CMV-infected cells, as well as the presence of T-lymphocytes and monocytes/macrophages were studied by histopathologic and immunohistochemical staining techniques. The meninges showed $\mathrm{CMV}$ infection in mononuclear infiltrating cells and in endothelium of small blood vessels eight days after intracerebral inoculation. This was accompagnied by multiple haemorraghes and inflammatory cell infiltration. The infection and inflammatory response persisted for at least 21 days p.i. Animals were treated with (S)-1-(3-hydroxy-2-phosphonylmethoxypropyl)cytosine (HPMPC), 9-(1,3dihydroxy-2-propoxymethyl)guanine (DHPG), hyperimmune serum (HIS) and both DHPG and HIS combined. Treatment with one dosage of HPMPC at $20 \mathrm{mg} / \mathrm{kg}$ effectively reduced virus titers. However, all other treatment modalities were not effective. In conclusion, the pathology of RCMV-induced encephalomeningitis in immunocompromised rats closely resembles that of AIDS patients. The infection is effectively treated with HPMPC. 


\section{Introduction}

Cytomegalovirus (CMV) infections are a major problem in immunocompromised patients especially in transplant recipients ${ }^{(314)}$ and in patients with the acquired immunodeficiency syndrome (AIDS). The prevalence of CMV infections in individuals infected with the human immunodeficiency virus (HIV) is high. $(109,136,416)$. In AIDS patients CMV infections are the most frequent opportunistic infections during the late phase of disease $(342,442,547)$. Those infections are associated with symptoms of fever, pneumonitis, hepatitis and retinitis ${ }^{(348)}$. A significant percentage of these patients $(254,474)$ experience neurological complications, such as encephalitis, meningo-encephalitis and radiculomyelitis $(21,338,452,474)$. Although it has been shown that neural tissue obtained from these patients contains CMV antigens or nucleic acids, the role of the virus in the pathogenesis of meningo-encephalitis is not fully understood $(160,370)$.

For treatment of CMV infections in these patients commonly used antiviral drugs are 9(1,3-dihydroxy-2-propoxymethyl)guanine (DHPG) and phosphonoformate (PFA) (144, 198). The use of both compounds is limited by serious side effects such as neutropenia and kidney failure. The effect of alternative treatment modalities like hyperimmune serum (HIS) in AIDS patients is not known.

More recently, it has been described that (S)-1-(3-hydroxy-2-phosphonylmethoxypropyl) cytosine (HPMPC) ${ }^{(125,126)}$ is a very potent inhibitor of herpes viruses ${ }^{(556)}$. The advantage of HPMPC over DHPG is the very high selectivety and antiviral activity against human CMV. Other advantages are that HPMPC is highly specific and active against rat and mouse CMV $(373,498,499)$. The intracellular halflive of the active metabolite is long and in animal studies the effect of HPMPC is long lasting $(373,498)$. In immunocompromised rats HPMPC completely inhibited viral replication in all internal organs and effectively reduced the mortality from RCMV infection.

In this report we describe CMV infection in rats at day 8 and day 21 p.i. after intracerebal infection of rat CMV (RCMV). We compared the therapeutic effect of HPMPC on this infection to the effects of DHPG, HIS and both HIS and DHPG combined.

\section{Material and methods}

\section{Animals}

Inbred specified pathogen free (SPF) male Brown Norway (BN) rats of 8 weeks old were bred at the department of experimental animal services at the University of Maastricht, The Netherlands. Animals were used for the experiment at a total body weight between 140 and $180 \mathrm{~g}$.

The rats were immunosuppressed one day before infection by 5 Gray total body irradation (TBI) as described previously (497). 
Table 1.

Experimental groups and treatment regimens used.

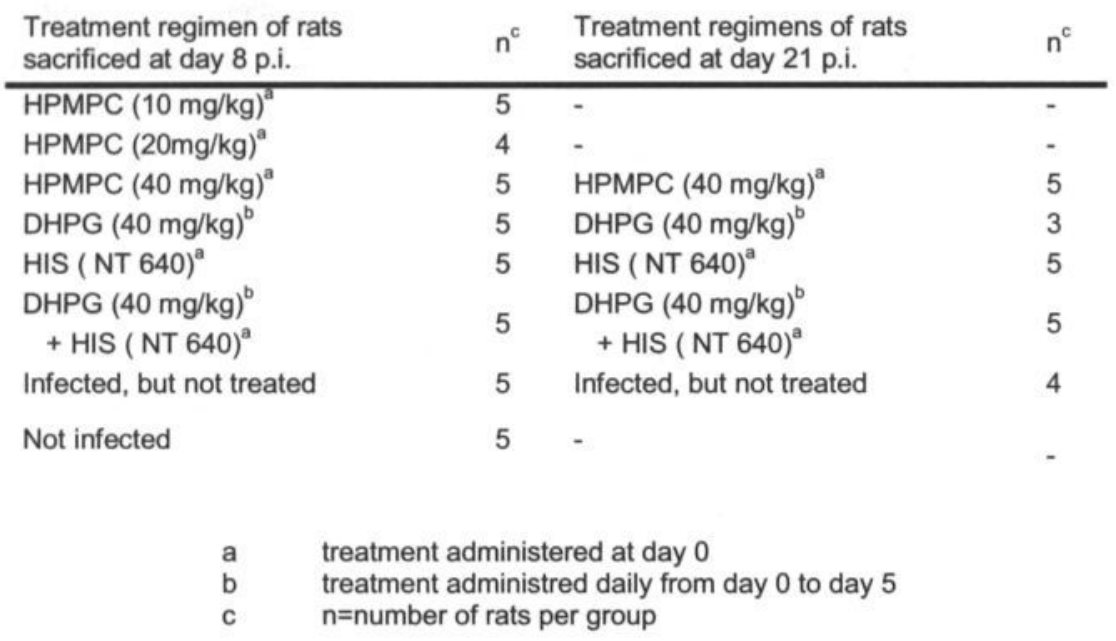

\section{RCMV infection}

The rat cytomegalovirus (RCMV) Maastricht strain, was obtained from a pool of salivary glands of infected laboratory rats $(75,78)$. The virus pool used underwent 7 passages in laboratory rats. The salivary glands were homogenised and the supernatant was collected after centrifugation and stored at $-70^{\circ} \mathrm{C}$. Each rat received $10^{5}$ plaque forming units (PFU) of RCMV in a volume of $20 \mu \mathrm{l}$ by intracerebral injection. Controls received $20 \mu \mathrm{l}$ of a noninfected salivary gland supernatant.

\section{Antiviral treatment}

The antiviral agents ganciclovir, 9-(1,3-dihydroxy-2-propoxymethyl)guanine was purchased from Syntex (Palo Alto, CA, USA). (S)-1-(3-hydroxy-2-phosphonylmethoxypropyl)cytosine (HPMPC) (Gilead Sciences, Foster City, CA, USA) was a gift from Dr E. De Clercq, Leuven, Belgium. Hyperimmune serum (HIS) was prepared in rats as described by Stals et al. 1990. In this study HIS was used at a neutralization titer of 640 and was given intravenously (i.v.). The antiviral compounds (HPMPC and DHPG) were administered by intraperitoneal (i.p.) injection. All treatments were started one day after RCMV infection. The treatment regimens used for each group are outlined in Table 1. 
All rats were examined daily for the presence of disease, as described by Stals et al. (497) and survival was recorded. To study the effect of treatment on the course CMV infection, brain tissue was obtained at two time points after infection (day 8 and 21 p.i.). To study the effect of treatment on the spread of virus within the body, the spleen was also sampled at these two time points. At that time the presence of infectious virus and viral antigens was measured. In addition, brain tissue was analysed for histopathological changes. The number of infectious virus particles determined in organs was counted by plaque assay using rat embryonic fibroblasts, as described previously ${ }^{(78)}$. The presence of viral antigens was evaluated by immunohistochemistry. For this purpose organs were fixed in paraformaldehyde-lysine-periodate and embedded in parafin. For detection of RCMV antigens mouse monoclonals (McAb) were used, directed against cytoplasmic (McAb 35) and nuclear (McAb 8) antigens (79). The infection load was graded by two independent observers based on the number of RCMV immunoreactive cells in the sections. This number was expressed as point score units (PSU), as described previously (310),

Table 2.

Effect of antiviral treatment on the RCMV infection in brain and spleen at day 8 post infection.

\begin{tabular}{|c|c|c|c|c|}
\hline \multirow{3}{*}{$\frac{\text { Treatment of rats }}{\text { HPMPC }(10 \mathrm{mg} / \mathrm{kg})}$} & \multirow{3}{*}{$\frac{n^{a}}{5}$} & \multicolumn{3}{|c|}{ Virus titer } \\
\hline & & Brain $^{b}$ & \multicolumn{2}{|c|}{ Spleen $^{b}$} \\
\hline & & $1.19(2.67)^{c}$ & $\overline{1.31}$ & $(1.80)^{c}$ \\
\hline HPMPC(20 mg/kg) & 5 & $0.72(1.44)^{c}$ & 0.00 & $(0.00)^{c}$ \\
\hline HPMPC (40 mg/kg) & 5 & $1.36(1.87)^{c}$ & 0.00 & $(0.00)^{\mathrm{c}}$ \\
\hline DHPG (40 mg/kg) & 5 & $6.58 \quad(0.22)$ & 4.64 & $(0.50)^{c}$ \\
\hline HIS (NT 640) & 5 & $6.79(0.18)$ & 4.73 & $(0.71)^{c}$ \\
\hline $\begin{array}{c}\text { DHPG (40 mg/kg) } \\
+ \text { +HIS (NT640) }\end{array}$ & 5 & $6.22(0.18)$ & 2.10 & $(1.95)^{c}$ \\
\hline none (control) & 5 & $6.54(0.47)$ & 6.05 & $(0.41)$ \\
\hline
\end{tabular}

\footnotetext{
a $\quad n=$ number of animals per group

b Virus titer in brain and spleen at day 8 p.i. was measured and is expressed as ${ }^{10} \mathrm{log}$ $\mathrm{PFU} / \mathrm{g}$ organ tissue (mean (standard deviation))

c Significant difference $(p<0.05)$ compared to the nontreated (control) group
} 
The quantity of CMV reactive cells scored from 0 to 5 ( 0 : no staining; 1 : sporadic cells; 2 : few cells; 3 : moderately dense; 4: dense; 5 : very dense). Parafin embedded sections of brain tissue $(4 \mu \mathrm{m})$ were analysed for the presence of inflammatory cells by the immunoperoxidase technique. Monoclonal antibody W3/13 (sera-lab, Crawly Brown, U.K.) were used for the detection of T-lymphocytes and monoclonal antibody ED-1 (kindly supplied by Dr. C. Dijkstra, University of Amsterdam, The Netherlands) for detection of monocytes and macrophages were used. In addition, routine haematoxylin and eosine staining was carried out.

\section{Statistical analysis}

The score of positive cells in different groups is expressed as median (range) and is compared with the aid of the two tailed non-parametric Mann-Whitney $U$ test. For comparison of the number of infectious virus particles measured by plaque assay the non parametric Mann-Whitney $U$ test was also used. $P$ values $<0.05$ were regarded as statistically significant.

Table 3.

Effect of antiviral treatment on the RCMV infection in brain and spleen at day 21 post infection.

Virus titer

\begin{tabular}{lcccc} 
Treatment of rats & $(\mathrm{n})^{\mathrm{a}}$ & \multicolumn{2}{c}{ Brain $^{\mathrm{b}}$} & \multicolumn{2}{c}{ Spleen $^{\mathrm{O}}$} \\
\hline HPMPC $(40 \mathrm{mg} / \mathrm{kg})$ & 5 & $1.26(1.76)^{\mathrm{c}}$ & $0.00(0.00)^{\mathrm{c}}$ \\
DHPG (40mg/kg) & 6 & $6.31(0.43)$ & $2.79(2.41)$ \\
HIS (NT 640) & 5 & $6.37(0.52)$ & $3.80(0.74)$ \\
DHPG+HIS & 5 & $5.84(0.61)$ & $3.72(0.30)$ \\
none (control) & 5 & $5.61(1.45)$ & $4.79(1.25)$
\end{tabular}

\footnotetext{
a $\quad n=$ number rats per group

b Virus titer in brain and spleen harvested at day 21 p.i. is expressed as ${ }^{10} \mathrm{log}$ $\mathrm{PFU} / \mathrm{g}$ organ tissue (mean (standard deviation))

c Significant difference $(p<0.05)$ compared to the nontreated (control) group
} 
Table 4.

Effect of antivirals on the presence of RCMV-infected cells within the meninges and in blood vessels within the meninges.

\section{Virus infected cells ${ }^{a}$ in}

\section{Blood vessels within the}

Meninges

\begin{tabular}{|c|c|c|c|c|}
\hline \multirow[b]{2}{*}{ Treatment } & \multicolumn{2}{|c|}{ Meninges } & \multicolumn{2}{|c|}{ meninges } \\
\hline & 8 p.i. & 21 p.i. & 8 p.i. & 21 p.i. \\
\hline $\mathrm{HPMPC}^{6}$ & $0.2(0.2)^{0}$ & $0.0(0.0)^{\sigma}$ & $0.0(0.0)^{\mathrm{d}}$ & $0.0(0.0)^{\mathrm{a}}$ \\
\hline $\mathrm{DHPG}^{\mathrm{b}}$ & $3.0(0.3)$ & $1.6(0.2)$ & $1.4(0.2)$ & $0.6(0.1)$ \\
\hline $\mathrm{HIS}^{\mathrm{c}}$ & $1.2(0.3)^{d}$ & $1.4(0.2)$ & $0.8(0.1)^{d}$ & $0.8(0.0)$ \\
\hline $\mathrm{HIS}^{\mathrm{c}}+\mathrm{DHPG}^{\mathrm{b}}$ & $2.8(0.2)^{d}$ & $2.4(0.3)$ & $1.6(0.3)$ & $2.8(0.4)$ \\
\hline None (control) & $3.7(0.3)$ & $1.7(0.4)$ & $2.2(0.3)$ & $1.2(0.1)$ \\
\hline
\end{tabular}

\footnotetext{
a Virus infected cells expressed as median (range)

b Drug administered at a dose of $40 \mathrm{mg} / \mathrm{kg}$

c $\quad 1 \mathrm{ml}$ of HIS (NT 640)

d Significant difference $\mathrm{p}<0.05$ compared to infected not treated (control) rats
}

\section{Results}

Effect of treatment on virus titers at day 8 p.i.

At 8 days after intracerebral inoculation of $10^{5}$ PFU RCMV, the brains harbored high numbers of infectious virus. In these animals generalised infection was observed by the presence of high numbers of infectious virus in the spleen (Table 2). Treatment with HPMPC at a dosage of 20 and $40 \mathrm{mg} / \mathrm{kg}$ significantly reduced the virus titers in both brain tissue and spleen. HPMPC treatment at $10 \mathrm{mg} / \mathrm{kg}$ reduced the virus titer in both organs, but not completely. The other treatment regimes (DHPG, HIS and both, DHPG and HIS combined) all failed to reduce the number of infectious $\mathrm{CMV}$ in the brains significantly. In contrast, these compound had a moderate effect on the number of infectious virus particles in the spleen.

Effect of treatment on the persistence of the virus in brain and spleen at day 21 p.i.

Intracerebral injection of RCMV resulted in persistence of infectious virus for at least a period of 3 weeks (Table 3). At that time CMV was also present in the spleen, indicating general infection during this period of disease. No significant difference was found in the infectious virus load between day 8 and day 21 , which indicates continuous viral replication 
in these immunosuppressed animals for a period of at least 3 weeks.

As described for day 8 p.i., HPMPC treatment at $40 \mathrm{mg} / \mathrm{kg}$ significantly decreased the number of RCMV in the brain. In addition, at that dosage HPMPC completely inhibited RCMV infection in the spleen, indicating that the drug prevented further spread of the infection through the body. The other treatment modalities (DHPG, HIS and both combined) did not significantly reduce virus titers in the brain and the spleen.

\section{Pathology of the brain and meninges and the effect of antiviral therapy}

CMV in meninges and brain tissue was localised immunohistochemically using RCMVspecific monoclonal antibodies. As presented in Table 4, the meninges of untreated rats harbored high numbers of virus-infected cells day 8 p.i. (cell score $=3.7(0.3)$ median (range) at day 8 p.i.). At 21 days p.i. the number of virus-infected cells declined to a score of 2 (1) median (range). In the brain tissue only few virus-infected endothelial cells and monocytes/macrophages were observed (median PSU (range) $1(0)$ at day 8 and $1(1)$ at day 21).

In the meninges, and to a significant lesser extent in the brain, scattered hemorrhages with foci of necrosis were present. CMV antigen was often detectable within these necrotic regions, as shown immunohistochemically. In the blood vessels numerous giant endothelial cells were noticed (data not shown). The enlarged cells contained basophilic intranuclear and intracytoplasmic inclusions which are typical for an active CMV infection. Immunohistochemical staining proved the presence of RCMV antigens in these cells.

While in non-infected rats virtually no inflammatory cells were noticed, in the CMVinfected animals numerous inflammatory cells infiltrated the meninges and brain. These infiltrates predominantly consisted of monocytes/macrophages and to a lesser extent Tlymphocytes. Nearly half of the macrophages present in the meninges and brain was reactive with monoclonals 8 and 35 , indicating that these cells contained viral antigens. Infiltrates were extensively present at day 8 p.i. Thereafter, the inflammatory response declined. In addition, endothelial cells, mainly those present in the small vessels, reacted with McAb 8 and 35.

The effect of treatment with HPMPC, DHPG, HIS and both, DHPG and HIS combined, on the quantity of virus-infected cells in brain and meninges was evaluated. As presented in Table 4, HPMPC at a dosage of $40 \mathrm{mg} / \mathrm{kg}$ significantly reduced the number of virusinfected cells in the meninges. In the blood vessels of these organs the effect was even more pronounced. In contrast, treatment with DHPG, HIS and both, DHPG and HIS combined, had hardly any effect on the number of CMV-infected cells. In the brains of untreated animals the number of virus-infected cells was low. Therefore, evaluation of the effect of antivirals was not performed. None of the four antiviral regimes had any effect on the inflammatory response in the meninges and in the brain of RCMV-infected rats. This 
was true for both measure points: day 8 and 21 p.i.

\section{Survival}

All animals submitted to this study survived at least during a period of 21 days. Only the HIS treated group and the control group became ill, but they all survived.

\section{Discussion}

This study describes the pathologic features of CMV infection in the brains and meninges of immunosuppressed rats after intracerebral inoculation of RCMV. In addition, it describes the effect of administration of antiviral treatment modalities, HPMPC, DHPG, HIS and both, DHPG and HIS combined, on the course of infection. The rat model described mimics the pathology of CMV-induced meningo-encephalitis in AIDS patients. Histochemical evaluation of brain and meninges from RCMV-infected rats revealed acute infection accompanied with signs of inflammation. Particulary, in the meninges high amounts of virus-infected cells were present. The virus-infected cells were localized mainly in and around the blood vessels and consisted predominantly of monocytes/ macrophages and endothelial cells. The presence of CMV-infected cells present in blood vessels of infected organs is also described in AIDS-patients (208). CMV infection in and around blood vessels is accompanied by a local inflammatory response. Earlier, it has been described that after inoculation of RCMV in the foot-pad of rats polymorphonuclear granulocytes infiltrated the perivascular region during the first days post infection, followed by infiltration of CMV-infected monocytes/macrophages. The inflammatory response reached the highest level at about one week after infection (399). Another interesting observation is the detection of viral antigens in the endothelium of meninges and brain during the acute phase of infection. The presence of CMV in the endothelium of the brain is also described in postmortem specimens of AIDS patients ${ }^{(360)}$. It suggests that endothelium could act as a portal for entry of the virus into the central nervous system. An important role of endothelial cells in the pathogenesis of CMV infection is suggested by (195), who indicates that infected endothelial cells are detectable in systemically infected immunocompromised patients. Predominantly, endothelial cells of small vessels become infected by CMV, while the endothelium of large vessels is exceptionally infected (486). Recent data of Vossen et al. (536) indicate that endothelial cells consist of a very heterogenous population that differently interact with CMV. Infection of these cells seems to be relevant for the pathogenesis. The finding of CMV specific antigens and nuclear inclusions of cells in the brain and meninges indicates local replication and is related with brain damage.

Treatment of RCMV-infected rats with DHPG, HIS or both DHPG and HIS combined had hardly any effect on the virus titers in spleen and brain of the animals. This finding is in contrast with the synergistic effect of DHPG and HIS during generalised infection $(440,499)$. 
In patients with severe CMV disease combined treatment with DHPG and immuneserum appears to be beneficial $(180,415)$. One explanation for therapeutic failure in this model could be poor penetration of the antivirals into the brain area, which permits initial replication cycles of the virus. It should also be mentioned that antibody does not cross the blood brain barrier unless there is inflammation. Since there is nearly no inflammatory respons in the brains this explains the fact that HIS alone or in combination with DHPG does not lead to a reduction in virus titer.

HPMPC treatment significantly reduced virus titers in brain and spleen. This is in agreement with earlier reports about the highly effective and specific effect of the drug ${ }^{(372,}$ 499). We found, using immunohistochemical methods, that CMV antigens were localised in endothelial cells and monocytes/macrophages of the brains and the meninges from rats. In contrast with the lack of antiviral effect of HIS and both HIS and DHPG combined, the number of virus-infected cells was reduced in the meninges at day 8 p.i. by these treatment regimens. Whether this effect was a result of a decreased number of infected cells, or a decreased sensitivity of the immunohistochemical detection technique by interference of the HIS, remains unknown.

High dosages of HPMPC reduced effectively and long lasting the virus titers as well as the number of virus-infected cells in both brain and spleen, while a lower dosage (10 $\mathrm{mg} / \mathrm{kg}$ ) of the drug showed a less pronounced effect. It should also be mentioned that intracerebral infection may injure the blood brain barrier and that this may possibly alter the penetration of the compound(s) in the brain. Yang et al. (556) demonstrated however that HPMPC is still able to reduce titers of HVS-2 in brains of mice that were injected intraperitoneally and in which treatment was only started at the time the virus had spread to the brains. Neyts et al. (371) demonstrated that HPMPC efficiently prevents MCMVassociated morbidity and mortality upon intracerebral infection. However, following longterm but infrequent treatment (once a week) with HPMPC in SCID mice that had been infected intraperitoneally with MCMV, virus replication was found in the brain. In contrast, little virus replication was found in other organs at that time. Thus brain infections may be more refractory to systemic treatment with HPMPC than visceral infections. This may suggest that perhaps higher and more frequent dosages of HPMPC are needed to treat brain infections with HPMPC than those doses used in the present study.

In conclusion, we showed that intracerebral RCMV infection in immunocompromised rats closely resembles severe human CMV infection in AIDS patients. It displays pathology with pronounced local infection of mononuclear infiltrates and endothelial cells of the small vessels. The treatment model seems suitable to study the effect of candidate antiviral drugs on CMV infection of meninges and brain in the severe immunocompromised host. HPMPC significantly reduces the viral load in brain, meninges and spleen. Therefore, we recommend to study the effect of HPMPC on CMV-induced encephalitis in AIDS patients. 


\section{Acknowledgements}

The authors would like to thank Wil Mullers for technical assistance and professor S. Wagenaar, Department of Pathology, for assistance in the evaluation of the immunohistochemical stainings. 


\section{Summary}

Cytomegalovirus (CMV) infection is a major problem in the immunosuppressed patient. It is thought that besides direct CMV induced cell lysis, immunological damage is part of CMV pathogenesis. New antiviral drugs, which combine immunomodulating and antiviral qualities, could be beneficial. Recently, it has been described that desferioxamine (DFO) and calcium trinatrium diethylenetriaminepentaacetic acid (DTPA) exhibit both properties. In this report the antiviral effects of both compounds against rat CMV (RCMV) infection are described in vitro and in vivo using a generalised and local infection model. In vitro, both compounds inhibited viral replication significantly, with DTPA being more potent than DFO. However, in the generalised infection model no effect was seen on mortality, morbidity or presence of virus in internal organs. In rats infected subcutaneously in the hind paw, no effect was seen locally on paw thickness, presence of viral antigens and inflammatory response. In addition, these rats suffered from a generalised infection of low magnitude at 15 days post infection. In this case both DFO and DTPA were able to lower the level of viral replication. In conclusion, our data indicate that despite previous in vitro reports, in vivo usage of DFO or DTPA as monotherapy for an acute CMV infection is questionable. The use of both compounds in combination with either ganciclovir or foscarnet should be tested in further studies. 


\section{Introduction}

Cytomegalovirus (CMV) infection is common in the general population. Clinical symptoms are observed in immunosuppressed patients. Since the onset of the Human Immunodeficiency Virus (HIV) epidemic, the number of patients suffering from a disseminated cytomegalovirus (CMV) disease has increased markedly. Currently, this progression has been halted by the use of Highly Active Antiretroviral Therapy (HAART) but nevertheless, it is still a major problem in medical health care. The brain, retina and the gastro-intestinal tract are frequently involved during CMV infection in these patients $(21,132$, 400). Infection may impair the patient's homeostasis drastically and may eventually evolve into a life threatening event. Also, in transplant (TX) patients and especially in heart TX recipients CMV infection is a major cause of morbidity and mortality (193). Similar outcomes are found in liver and kidney TX recipients $(294,379,407,423)$ and bone marrow TX (BMTX) patients (144).

Attempting to control the CMV induced pathology several treatment regimens have been developed. Nowadays, mostly 9-(1,3-dihydroxy-2-propoxymethyl)guanine (ganciclovir) or trisodium phosphonoformatic acid (foscarnet) are the treatment modalities of choice for combating CMV disease. In BMTX patients, ganciclovir is able to prevent disseminated CMV disease when the patient is treated prophylactically (188). Both compounds have been shown to drastically improve $\mathrm{CMV}$ retinitis in immunocompromised patients although relapses are common when treatment is stopped $(25,158,462,542)$. In 1986, De Clercq et al. (125) described a nucleotide analogue (s)-1-(3-hydroxy-2phosphononylmethoxypropyl)cytosine (HPMPC, cidofovir) which is active against various DNA viruses, including CMV. Cidofovir has been tested in animal models $(371,498,500)$ and is now available for treating patients (299).

Despite the strong antiviral capacities of these compounds, usage is limited due to unwanted side effects. One of the major side effects of ganciclovir is neutropenia especially when ganciclovir is given to patients receiving other drugs such as trimethoprimsulfamethoxazole (190) or azidothymidine (AZT) ${ }^{(430)}$. Anemia, proteinuria and an elevated serum creatinin level indicating nephrotoxicity are adverse effects of foscarnet and cidofovir treatment. Besides this, foscarnet should be administered intravenously hereby limiting its clinical use (542).

Another complicating factor is that some strains of CMV are less sensitive or even resistant to ganciclovir $(135,146,181)$ and to a lesser extent to foscarnet $(238,307)$. Alarming are the reports of Sarasini et al. (448) and Baldanti et al. (29) who described multiple CMV strains resistant to both ganciclovir and foscarnet. In addition, also a cidofovir resistent strain has been described in vitro (299) and in vivo (206).

Recently, progress has been made in the search for new antiviral drugs. Cinatl et al. described two compounds desferioxamine (DFO) and calcium trinatrium 
diethylenetriaminepentaacetic acid (DTPA), which were able to inhibit human CMV replication in vitro $(103,105)$. Both compounds are metal chelators and clinically DFO is already used in case of an iron overload (404). Besides data of their antiviral capacities in vitro also an in vivo effect is described in an AIDS patient suffering from a progressive $\mathrm{CMV}$ retinitis. This patient did not respond to combination therapy consisting of ganciclovir and foscarnet but when DFO was added to the treatment regimen, the progression was halted (201).

Although the cited references suggest an antiviral effect of DFO and DTPA on human $\mathrm{CMV}$ replication in vitro, hardly any data are available concerning the antiviral properties of both compounds on in vivo replicating virus. For this purpose we used a rat model and the appropriate virus (rat CMV, RCMV) and studied the effects of DFO and DTPA on viral dissemination after systemic or local inoculation of virus.

\section{Materials and methods}

Animals and immunosuppression

Specific pathogen-free (SPF) Lewis rats were used in the in vivo experiments. The animals were bred at the Department of Experimental Animal service at the University of Maastricht, the Netherlands. Rats were kept under normal housing conditions and were fed normal rat chow ad libitum. Depending on the experiment, animals were used at an age of $4(60-90 \mathrm{~g}), 6(120-140)$ or $8(180-200 \mathrm{~g})$ weeks. In order to enhance the effect of the infection rats received a total body irradiation (TBI) of 5 Gy 8 hours before infection as discussed by Stals et al. (497).

\section{Virus, infection and treatment}

Rats infected intraperitoneally (i.p.) were given $3 \times 10^{5}$ plaque forming units (PFU) of RCMV. The RCMV inoculum was derived from a pool of salivary glands from acutely infected rats as described previously (75). Local infection was established by injecting rats

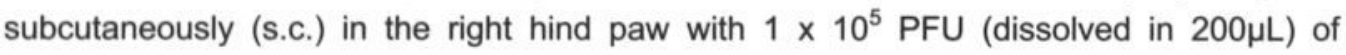
RCMV, which had undergone several in vitro passages. The left hind paw was injected with supernatant of uninfected fibroblasts $(200 \mu \mathrm{L})$ and served as an internal control.

Calcium trinatrium diethylenetriaminepentaacetic acid (DTPA) purchased from Heyl, Berlin, Germany and desferioxamine (DFO) purchased from Ciba-Geigy, Wehr, Germany were tested for their antiviral effect. For in vivo experiments both compounds were dissolved in distilled water and were given i.p. in a dose of $100 \mathrm{mg} / \mathrm{kg}$ dissolved in $0.2 \mathrm{ml}$ twice a day. Control (non-infected) rats received sterile Phosphate Buffered Saline (PBS) twice a day. 
For the in vivo experiments two models depending on the mode of inoculation are used. Intraperitoneal inoculation will lead to a fast dissemination of virus in the body as described earlier (497). A subcutaneous infection in the hind paw, however, will concentrate the viral load to the hind paw with slow release of virus to the systemic circulation (77, 399). In the i.p. infected rats a lethal and a sub-lethal infection was established by infecting 4 and 8 weeks old rats with $3 \times 10^{5}$ PFU of RCMV, respectively.

All rats were weighed and checked for CMV induced illness daily and survival was recorded. The sub-lethally infected rats were sacrificed at 4 and 21 days post infection (p.i.). Effect of treatment was determined in the sub-lethal experiment by measuring viral load by plaque assay and viral DNA by polymerase chain reaction (PCR) in important target organs such as salivary gland, spleen and liver. In the second model a local infection was established in the rat hind paw using rats of 6 weeks old (399). The paw thickness of the right and left hind paw was measured at a fixed point on both hind paws on a daily basis. Rats were sacrificed at 4,8 and 15 days p.i. Effect of DFO or DTPA on the presence of viral antigens in the rat hind paw and on the amount of infectious virus in internal organs was measured. In addition the effect of treatment on local inflammatory response (paw thickness and presence of inflammatory cells) was followed.

\section{Plaque assay and PCR}

The amount of infectious virus was determined by plaque assay using rat embryonic fibroblasts (REFs) as described before (78) and is expressed as the number of PFU/g tissue homogenate.

Presence of viral DNA in the extracted organs was determined by a semiquantitative nested PCR as described previously (525).

\section{Immunohistochemistry}

Harvested tissues were fixed in a 3.7\% formaldehyde solution in PBS for 24 hours and embedded in paraffin. RCMV antigens were detected on $4 \mu \mathrm{m}$ tissue sections with a mixture of 2 mouse monoclonal antibodies (MoAb) 8 and 35 (79). MoAb W3-13 (Sera-lab, Crowley Down, UK) reacting with rat T-lymphocytes and MoAb ED-1 (gift from Dr. C. Dijkstra, Amsterdam, The Netherlands) specific for rat monocytes and macrophages were used to characterise infiltrated cells. Expression of interleukin-2 receptor (IL-2R) reflecting T-cell mediated immune activation was determined using the appropriate antibody (OX39, Sera-lab, Crowley Down, UK). Positive cells were detected by using the immunoperoxidase method as described earlier ${ }^{(497)}$. Slides were evaluated semiquantitatively (- = no positive cells, $-l_{+}=$sporadic cells, $+=$few cells, $++=$average number of cells, $+++=$ many cells, ++++ = very many cells) by two independent observers. 

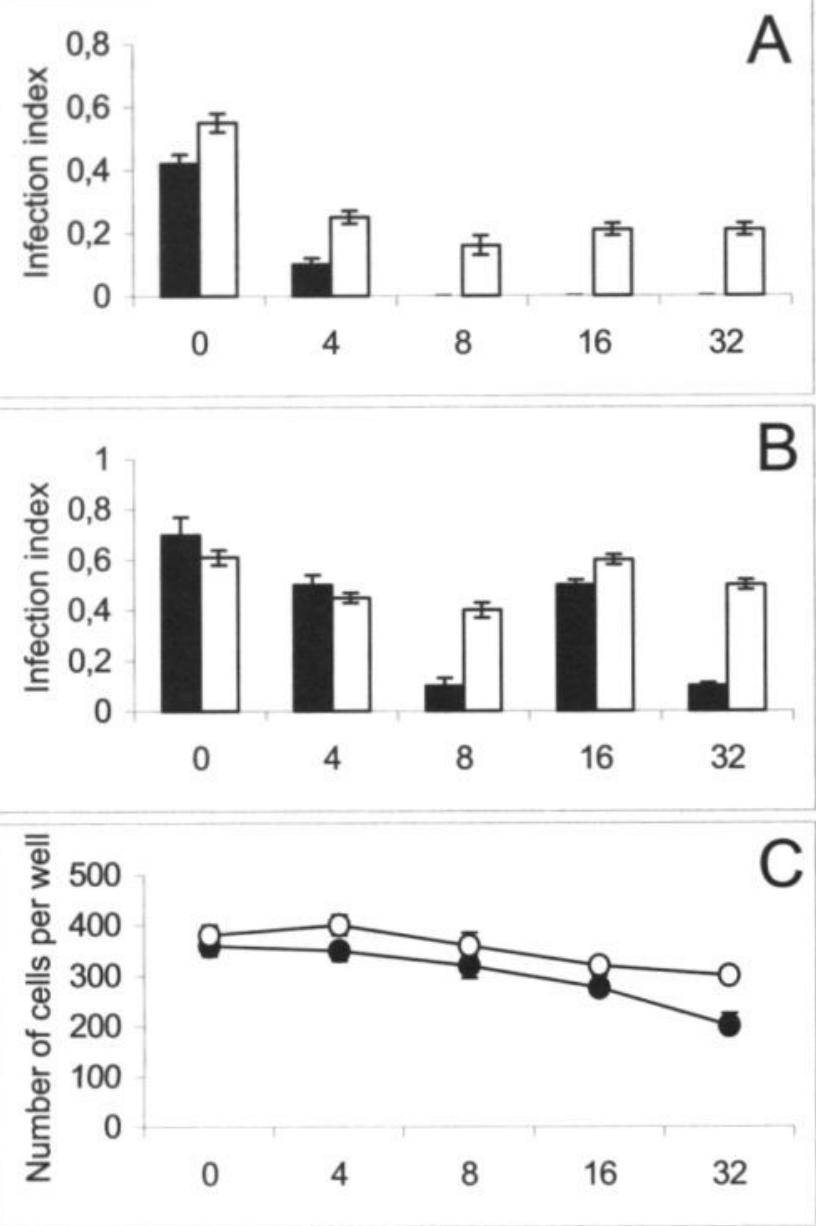

Figure 1. Antiviral properties of DTPA (indicated in black) and DFO (indicated in white) in vitro. REFs were infected with RCMV at a $\mathrm{MOI}$ of 0.01 and fixed at 5 days p.i. (figure $\mathrm{A}$ ) or infected with a MOI of 0.1 and fixed at 3 days p.i. (figure B). Viral nuclear antigens were stained with MoAb8. The bar charts show the ratios between infected versus total number of counted cells (infection index). The line chart (figure $\mathrm{C}$ ) shows the number of uninfected cells treated with DFO or DTPA. Control cells were fixed at 3 days p.i. 


\section{Table 1}

Presence of infectious virus ${ }^{a}$ in organs of intraperitoneal infected rats ${ }^{b}$ with and without drug treatment.

\begin{tabular}{lcccccc} 
Treatment & \multicolumn{2}{c}{ Salivary gland $^{c}$} & \multicolumn{2}{c}{ Spleen ${ }^{d}$} & \multicolumn{2}{c}{ Liver $^{d}$} \\
\hline DFO $^{e}$ & $7.63 \pm 1.6^{9}$ & $5 / 5^{\mathrm{h}}$ & $1.04 \pm 1.43$ & $7 / 7$ & $0.00 \pm 0.00$ & $0 / 7$ \\
DTPA $^{\mathrm{e}}$ & $7.98 \pm 1.5$ & $5 / 5$ & $2.50 \pm 1.42$ & $7 / 7$ & $0.00 \pm 0.00$ & $0 / 7$ \\
None $^{\mathrm{f}}$ & $8.65 \pm 0.6$ & $5 / 5$ & $0.00 \pm 0.00$ & $0 / 7$ & $0.00 \pm 0.00$ & $0 / 7$
\end{tabular}

\footnotetext{
a Measured by plaque assay

b Rats received $3 \times 10^{5}$ PFU RCMV by intraperitoneal injection

c Harvested at day 21 post infection ( $n=5 /$ group)

d Harvested at day 4 post infection ( $n=7 /$ group)

e Compound given intraperitoneally at a dose of $100 \mathrm{mg} / \mathrm{kg}$ twice a day

f Control rats received $0.2 \mathrm{ml}$ PBS twice a day intraperitoneally

$g \quad$ Expressed as ${ }^{10} \mathrm{log}$ PFU per gram tissue

h Number of rats harbouring infectious virus per total number of rats.
}

\section{Antiviral properties of DTPA and DFO}

For detection of an antiviral effect in vitro an immunofluoresence assay was used. For this purpose REFs were cultured in 96 well culture dishes and were used at confluency. RCMV was added at a multiplicity of infection (MOI) of 0.01 or 0.1 . For optimal infection the cells were centrifuged at $700 \mathrm{~g}$ for 45 minutes at $20^{\circ} \mathrm{C}$. Directly after infection, cells were treated with DTPA or DFO at the concentrations of $0,4,8,16,32,64,128$ and 256 $\mu \mathrm{M}$. Cells were fixed at 3,5 or 7 days post infection and the presence of RCMV antigens was detected by indirect immunofluorescence using RCMV specific monoclonal antibodies as described above. Cellular DNA was counterstained with $0.5 \mu \mathrm{g} / \mathrm{ml}$ 4'-6-diamino-2phenyl indole (DAPI). The number of RCMV positive cells per well was scored by counting 5 visual fields ( $200 \times$ magn.) and the ratio of the number of positive cells over the total number of cells (infection index) was determined. Each experiment was performed in quintuplo.

\section{Statistical analysis}

In all experiments the two sided non parametrical Mann Whitney $U$ test was used and $p<0.05$ was regarded as significantly different. 
Table 2.

Presence of infectious virus ${ }^{a}$ in organs of locally RCMV infected rats ${ }^{b}$ with and without drug treatment.

\begin{tabular}{llllllllll} 
& \multicolumn{1}{l}{ Liver } & \multicolumn{4}{c}{ Spleen } & \multicolumn{3}{c}{ Salivary gland } \\
\cline { 2 - 9 } & 4 & 8 & 15 & 4 & 8 & 15 & 4 & 8 & 15 \\
\hline DFO $^{c}$ & $0 / 5$ & $0 / 5$ & $1 / 5$ & $0 / 5$ & $0 / 5$ & $0 / 5$ & $0 / 5$ & $0 / 5$ & $5 / 5$ \\
DTPA $^{c}$ & $0 / 5$ & $0 / 5$ & $0 / 3^{1}$ & $0 / 5$ & $0 / 5$ & $0 / 3$ & $0 / 5$ & $0 / 5$ & $3 / 3$ \\
Control $^{d}$ & $0 / 5$ & $0 / 5$ & $5 / 5$ & $0 / 5$ & $1 / 5$ & $5 / 5$ & $0 / 5$ & $0 / 5$ & $5 / 5$
\end{tabular}

a Measured by plaque assay

b Rats were infected with $1 \times 10^{5} \mathrm{PFU}$ in the subcutis of the hind paw

c Compound given intraperitoneally at a dose of $100 \mathrm{mg} / \mathrm{kg}$ twice a day

d Control rats received $0.2 \mathrm{ml}$ PBS twice a day intraperitoneally

e Expressed as number of rats harbouring infectious virus per total number of rats

f Significant $(p<0.05)$ when compared to control rats.

\section{Results}

In vitro

Viral inhibition and cytotoxity. Confluent REFs were infected with a $\mathrm{MOI}$ of 0.01 and were fixed at day 5 p.i. respectively. Addition of DTPA and DFO at a concentration of $4 \mu \mathrm{M}$ to cells infected at a $\mathrm{MOI}$ of 0.01 resulted in a significant reduction of the infection index when compared to control (i.e. non treated) cells (figure 1A). When DTPA was used at a concentration of $8 \mu \mathrm{M}$ a complete reduction in the number of RCMV infected cells was found. In contrast, infection index seen with DFO treatment did not change at this concentration. Even when high concentrations (16 and $32 \mu \mathrm{M})$ of DFO were used, no effect was seen.

The antiviral effect of DFO and DTPA was dependent on the concentration of input virus as is shown in figure 1B. REFs were infected at a $\mathrm{MOI}$ of 0.1 and fixed at day 3 p.i. At this $\mathrm{MOI}, \mathrm{DFO}$ was not able to decrease infection index significantly at a concentration of 4 $\mu \mathrm{M}$. However, a significant reduction in infection index was observed in cells treated with 8 $\mu \mathrm{M}$ of DTPA $(0.9 \pm 0.1)$ when compared to control cells $(0.7 \pm 0.1)$. Cellular cytotoxicity was determined by counting the number of (non-infected) cells treated with DFO or DTPA at various concentrations. The concentration at which the compounds gave a $50 \%$ reduction in cell number (CC50\%) was calculated. For DTPA the CC50\% were 150, 52 and $20 \mu \mathrm{M}$ after 3,5 or 7 days of incubation respectively, while for DFO the CC50\% were 288,100 and $84 \mu \mathrm{M}$. 
As shown in figure $1 \mathrm{C}$, the number of cells did not change when concentrations of $\overline{D F O}$ up to $16 \mu \mathrm{M}$ were used after 3 days of culture in the presence of DFO. For DTPA no effect on the number of cells was observed when concentrations up to $8 \mu \mathrm{M}$ were used (after 3 days of culture). At these concentrations both compounds exhibited their antiviral effect as described above.

\section{In vivo}

\section{Lethal intraperitoneal infection}

To investigate whether DTPA and DFO could avert a lethal CMV challenge, immunosuppressed rats of 4 weeks old were inoculated i.p. with a lethal dose of $3 \times 10^{5}$ PFU of RCMV as described before (497). At 6 days p.i., rats started to show symptoms of CMV disease, such as subcutaneous and mucosal haemorrhages, development of ascites, hypokinesia and weight loss. The increase in weight per day during the experiment was $1.6 \mathrm{~g}, 2.0 \mathrm{~g}$ and $2.6 \mathrm{~g}$ for DFO treated, DTPA treated and control rats, respectively. There was no significant difference between the DFO and DTPA treated and the control rats in severity of clinical symptoms and in time of onset of these symptoms. Infection led to death starting at 9 days after infection in 4 out of 6 control rats, in 6 out of 7 DFO treated and in 5 out of 7 DTPA treated rats. No significant difference could be detected in the time of death. Rats surviving the CMV inoculation were followed for a period of 2 weeks and all these animals recovered completely.

\section{Sublethal intraperitoneal infection}

Presence of virus in liver, spleen and salivary gland. In order to investigate the antiviral properties of DTPA and DFO in rats suffering from a generalised infection, rats of 8 weeks old were i.p. infected and the effect of DFO or DTPA treatment on viral replication in internal organs was investigated. As is shown in table 1 , salivary gland homogenates yielded high amounts of infectious virus in non treated animals $(8.65 \pm 0.6 \mathrm{log} \mathrm{PFU} / \mathrm{ml})$ at 21 days p.i. Although treatment with DFO or DTPA showed a slight decrease in viral load ( $7.63 \pm 1.6$ and $7.98 \pm 1.5$ respectively) in this organ, this decrease was not significant. In spleens harvested at day 4 p.i. a low amount of infectious virus could be detected in DFO or DTPA treated rats while in the non treated rats no infectious virus was detectable in this organ. No infectious virus could be detected in spleen samples harvested at day 21 and in all liver samples.

Presence of viral DNA in organ samples was determined semiquantitatively. Spleen and liver samples yielded viral DNA in $10^{-5}$ and $10^{-1}$ dilutions at day 4 p.i., respectively. In salivary gland samples harvested at day 21 p.i. viral DNA was found in $10^{-8}$ dilutions. No differences were seen in amount of viral DNA between non treated, DFO treated or DTPA treated rats (data not shown). 
Detection of viral antigens by immunohistochemistry showed that RCMV positive cells were present in the salivary glands in all experimental groups at 21 days p.i. Treatment with DFO and DTPA did not result in a reduction of the number of RCMV positive cells. In conclusion, using the intraperitoneal infection route, no antiviral effect was observed in DFO and DTPA treated animals when compared to controls.

\section{Subcutaneous infection in the hind paw}

Macroscopy. As depicted in figure 2, in this infection model the course of infection can be divided in 4 phases. Phase 1 (day 1-5) is the steady state with no increase in paw thickness. Phase 2 (day 6-9) is characterised by a sharp increase in thickness and massive inflammation. Although the infected paw was painful, functionality was preserved until 12 days p.i. The next phase (day 9-12) is the plateau phase in which loss of functionality and edema of the leg are the most eminent phenomena. In this period the inflamed hind paw starts to loose its hair indicating tissue destruction. The last phase (day 13-15) is the healing period in which paw thickness and inflammation gradually decrease. In this period also functionality returns. The observed effects are all RCMV induced, since mock infection in the left paw did not show any sign of inflammation or increase in paw thickness. Treatment with DTPA or DFO did not lead to a significant reduction in paw thickness increase (figure 2).

Viral dissemination from the hind paw infection locus. Subcutaneous injection of RCMV led to a generalised infection as shown by the presence of infectious virus in spleen, liver and salivary gland at 15 days p.i. (table 2). At 4 and 8 days p.i. no infectious virus was detected in all organ samples except in the spleen of one animal. At day 15 p.i. all liver and spleen samples of the infected (non treated) rats contained RCMV. Treatment with DFO or DTPA reduced the number rats harbouring infectious virus in both the spleen and liver at 15 days p.i.

Plaque assays of the salivary glands extracted at 4 and 8 days p.i. remained negative. However, at day 15 p.i. the salivary glands yielded high amounts of infectious virus and in contrast to the spleen and liver no effect of antiviral therapy on viral replication was seen in this organ.

In spleens of control and DFO treated rats viral DNA was found in $10^{-2}$ dilutions whereas spleen samples of DTPA treated rats remained negative at 15 days p.i. In liver and salivary glands no differences in RCMV DNA content were found between DFO treated, DTPA treated and control rats. Viral DNA was present in $10^{-2}$ dilutions for liver samples and in $10^{-9}$ dilutions for salivary gland samples respectively. 
Table 3.

Table showing the inflammatory response at 4,8 and 15 days after

RCMV injection in the rat hind paw.

\begin{tabular}{|c|c|c|c|c|c|c|}
\hline & \multicolumn{3}{|c|}{$8835^{\circ}$} & \multicolumn{3}{|c|}{ W3-13 } \\
\hline & 4 & 5 & 15 & 4 & 8 & 15 \\
\hline $\mathrm{DFO}^{\prime}$ & $4^{3}$ & $++t$ & ++ & + & $+1++$ & ++4 \\
\hline DTPA' & + & $++t$ & $+t$ & + & $+1+t$ & +++ \\
\hline \multirow[t]{3}{*}{ none } & + & +++ & $+t$ & + & $+1++$ & +++ \\
\hline & \multicolumn{3}{|c|}{$E D-1^{a}$} & \multicolumn{3}{|c|}{$1 \mathrm{~L}-2 \mathrm{R}^{\mathrm{e}}$} \\
\hline & 4 & 8 & 15 & 4 & 8 & 15 \\
\hline $\mathrm{DFO}^{\circ}$ & + & ++ & ++++ & $1+$ & + & $+1++$ \\
\hline DTPA ${ }^{f}$ & + & ++ & $+t+t$ & $-1+$ & + & $+1++$ \\
\hline none ${ }^{9}$ & + & ++ & ++++ & $-1+$ & + & $+1+t$ \\
\hline
\end{tabular}

$\begin{array}{ll}\text { a } & \text { Rats were subcutaneously infected with } 1 \times 10^{5} \mathrm{PFU} \\ \text { b } & \text { Cells reactive with MoAb } 8 \text { \& } 35 \text {, specific for RCMV early antigens } \\ \text { c } & \text { Cells reactive with MoAb W3-13, specific for T-lymphocytes } \\ \text { d } & \text { Cells reactive with MoAb ED-1, specific for monocytes/macrophages } \\ \text { e } & \text { Cells expressing the Interleukin- } 2 \text { receptor } \\ \text { f } & \text { Compound given intraperitoneally at a dose of } 100 \text { mg/kg twice a day } \\ \text { g } & \text { Control rats received } 0.2 \text { ml PBS twice a day intraperitoneally } \\ \text { h } & \text { The presence of cells was semiquantitatively analysed and } \\ & \text { expressed as median (- through }++++) .\end{array}$

Although infectious virus and viral DNA could be detected in liver and spleen, no RCMV induced antigens were found in these organs by immunohistochemistry. Only the salivary glands at 15 days p.i. yielded positive staining. DFO or DTPA treatment did not result in a decline in the number of cells expressing viral antigens.

Viral antigen detection in the hind paw. After staining with MoAb 8 and 35, the amount of viral antigens was determined. In the hind paw of RCMV infected rats, cells with a cytoplasmic staining were observed in the hypodermis and the reticular layer of the dermis. In control, i.e. non treated, animals the amount of viral antigens could be correlated to the macroscopic findings in the hind paw as described above. In the phase before onset of paw thickness increase, the amount of RCMV positive cells was low $(-/+)$. Maximal increase in paw thickness correlated with a large number of infected cells $(+++)$ at day 8 p.i. In the last phase (i.e. 15 days p.i.), when paw thickness decreased, also the presence of virus decreased, leading to a low level (+) of RCMV positive cells. Treatment with DFO or DTPA could not reduce the number of viral antigen positive cells when compared to control rats. 


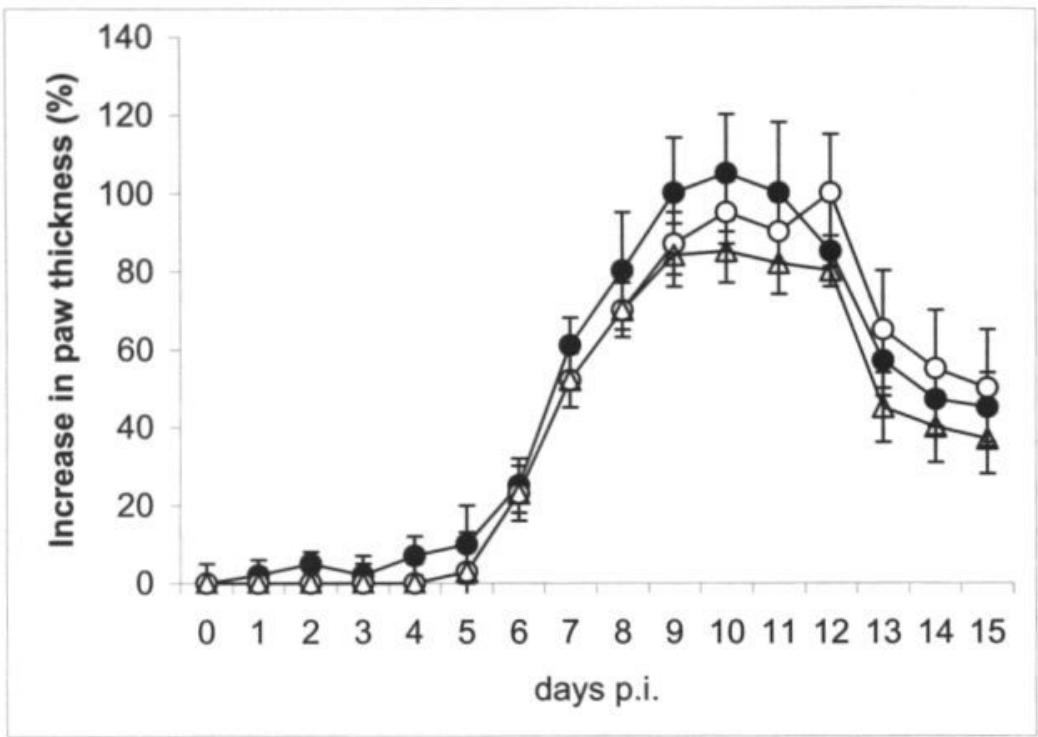

Figure 2. Paw thickness of rats after sub-cutaneous injection of $1 \times 10^{5} \mathrm{PFU}$ RCMV in the dorsum of the right hind paw. (day 0 to $4: n=15 /$ group, day 5 to 8 : $\mathrm{n}=10 /$ group, day 9 to $15: \mathrm{n}=5 /$ group). Black triangle indicates $100 \mathrm{mg} / \mathrm{kg}$ DTPA i.p. twice a day; White circle indicates $100 \mathrm{mg} / \mathrm{kg}$ DFO i.p. twice a day; black circle indicates $0.2 \mathrm{ml}$ PBS i.p. twice a day.

Inflammatory response. Influx of T-lymphocytes and monocytes/macrophages at the inoculation site gradually increased and the amount of both cell types present reached a maximum at 15 days p.i. Also the expression of IL-2R increased over time until day 15 p.i. No differences were found in the amount of T-lymphocytes, monocytes/macrophages and expression of IL-2R between DFO treated, DTPA treated and control rats (table 3 ).

\section{Discussion}

Recently it has been reported that DFO (103) and DTPA (104) are able to reduce CMV replication in human cells. In a case report, Gumbel et al. (201) have shown that the antiviral effect was also observed in vivo. In order to further study this effect in vivo a well defined animal model with the appropriate virus (RCMV) was used. Our in vitro data are in agreement with the previous reports on human cells and indicate that both DFO and DTPA are able to lower viral replication. However, only DTPA could reduce the amount of infected cells completely. Bearing in mind previous results $(103,105)$ one could state that the magnitude of the decrease of infection index by DFO and DTPA was dependent on the viral load. 
In vivo, two infection models, i.e. the intraperitoneal and the subcutaneous infection model were used. With the intraperitoneal infection model characterised by a fast spread of the virus into internal organs, the effect of both compounds on dissemination of virus was studied. By using the local, i.e. subcutaneous infection model, it was possible to study not only the spread of the infection in the host but also to follow the local inflammatory response. The latter is important in the case of DFO and DTPA since it is known that both compounds have anti-inflammatory effects $(450,451)$.

In the generalised (intraperitoneal) infection model no effect of DFO and DTPA was observed, neither on morbidity and mortality nor on the presence of virus in internal organs. The salivary gland is the preference organ for this virus and contains high amounts of infectious virus. In this organ, no effect of either both compounds was observed in all animals tested. In contrast, in the local infection model an antiviral effect, as shown by a decrease in the number of rats harbouring infectious virus in liver and spleen at day 15, was found. Also no viral DNA in spleens of DTPA treated rats could be detected. The difference in effect in both models could be due to differences in viral load: in spleen samples of control (i.e. non treated) rats, viral DNA present in the intraperitoneally infected rats exceeded the amount of viral DNA detected in the subcutaneously infected animals. Besides the viral load, the difference in effect might also be explained by biological variations.

The mechanism by which both compounds act has not been fully elucidated but it is thought that the inhibition of an iron dependent ribonucleotide reductase plays an important role in inhibiting RCMV replication as was shown for other herpes viruses (489). In order to evaluate the effect of DTPA and DFO on the inflammatory response, paw thickness was measured daily and the number of inflammatory cells (T-lymphocytes and monocytes and macrophages) and the IL-2R expression reflecting T-cell mediated immune activation in the hind paw was determined. In control (i.e. non treated) animals the observed inflammatory response was consistent with the previous report of Persoons et al. (399). Treatment with DFO or DTPA did not diminish the amount of T-lymphocytes, monocytes/macrophages nor the expression of IL-2R. Also macroscopically, both compounds were not able to reduce the increase in paw thickness. The latter is in contrast to the report of Blake et al. (53) in which a bolus of $30 \mathrm{mg}$ DFO i.p. (instead of $100 \mathrm{mg} / \mathrm{kg}$ i.p. twice a day used in our experiments) was able to reduce foot pad swelling after monosodium urate monohydrate cristal or carrageenan injection in the rat foot pad. In addition to the inhibitory actions of iron depletion on viral replication as discussed above, iron itself has been associated with increase in free radicals resulting in an increase in oxidative stress ${ }^{252)}$. In auto-immune uveitis this increase in oxidative stress will lead to retinal tissue destruction. The magnitude of retinal cell loss can be diminished by DFO treatment (555). It is not known why both DFO and DTPA failed to have an effect on the 
inflammatory response in the hind paw. However, one should bear in mind that immunologically induced damage and direct viral induced cell lysis are not totally separable. For it is known that CMV infection not only increases intracellular reactive oxygen intermediates (ROIs), but also benefits from a ROls mediated enhancement of viral gene expression and thus viral replication (491). Both the viral replication and the inflammatory process could result in a vicious circle. This could account for the differences in the observed immunomodulatory capacities of DFO and DTPA between our data and the papers of Wu et al. (555) and Kadiiska et al. (252).

Recently Gumbel et al. reported that addition of DFO to conventional treatment was able to reduce CMV induced retinitis refractory to ganciclovir and foscarnet in an AIDS patient (201). It is unclear whether the improvement of visual acuity was the result of the immunomodulatory or the CMV inhibiting capacities of the drug or a combination of both.

In conclusion, our data indicate that despite previous in vitro reports, in vivo usage of DFO or DTPA as monotherapy for an acute CMV infection is questionable. The use of both compounds in combination with either ganciclovir or foscarnet should be tested in further studies.

\section{Acknowledgements}

The authors would like to thank Bieke Vanherle, Monique Coomans, Patrick Beisser and Wil Mullers for their excellent technical assistance and Joanne Van Dam for carefully reading the manuscript. 
Chapter 8

\section{Summary and general discussion}




\section{Introduction}

The pathophysiological and clinical characteristics of CMV infection are reviewed in chapter 1. Seroprevalence of CMV infections in the normal population is high and CMV infection usually follows a subclinical course. In contrast, patients with a defective immune system are at risk for developing severe CMV disease. For example, AIDS patients with a progressive decline in CD4+ T cell counts, suffer from severe CMV infections at CD4+ T cell counts below 100 cells/ $\mu$ l blood. Additionally, when immune effector functions are restored by HAART, the incidence of opportunistic infection, such as CMV, drastically diminishes.

$\mathrm{CMV}$ is able to infect a wide range of tissues and one of the main characteristics of $\mathrm{CMV}$ infection is the ability to persist at these sites, i.e. during chronic infection or latency. Peripheral blood mononuclear cells and their progenitors have been suggested as the main site of viral latency in humans, although CMV DNA can be found in multiple cell types in several organs of healthy trauma victims. Allogeneic stimulation of latently infected peripheral blood mononuclear cells has been shown to result in reactivation of virus. In addition, CMV seropositive patients receiving hemapoietic stem cell transplantation are at the highest risk for developing CMV disease, regardless of the CMV serostatus of the donor. These findings stress the importance of immune activation on RCMV replication and reactivation. Data from the murine model suggest that reactivation of virus from the lungs occurs stochastically via a patchwork pattern and does not depend on a decrease in immune effector functions. The significance of these findings for the human situation remains to be determined.

The interaction between CMV and the host's immune system is complex. It is generally accepted that atherosclerosis is an chronic inflammatory disease and develops as a response to injury. There is increasing evidence that systemic CMV infection or CMV replication in the atherosclerotic vascular wall itself accelerates atherosclerotic changes. CMV-mediated endothelial dysfunction and virus-mediated proliferation and migration result in increased inflammation, lipid accumulation and lumen narrowing. Additionally, $\mathrm{CMV}$ is thought to be an important risk factor for chronic rejection. The development of chronic rejection in the CMV-infected host is mediated by an increased and prolonged immune response, which is triggered by the viral challenge. On the other hand, however, TNF- $\alpha$ has been shown to directly enhance CMV replication via stimulation of the CMV IE enhancer/promotor mediated by transcription factor NF-кB. These data suggest that CMV replication enhances inflammation and vice versa.

Current treatment of CMV infections with either ganciclovir, foscarnet or cidofovir is effective, although drug resistant strains have been reported. Furthermore, evidence is increasing that also CMV-triggered inflammation may partly account for the observed tissue damage. This points out the need for new antiviral therapies in which antiviral 
activity and the ability to modulate CMV-triggered immune activation are combined. Insight in viral pathogenesis is essential for developing new modalities of intervention. RCMV has been isolated approximately 20 years ago ${ }^{(78)}$ and experimental RCMV infections have been accepted as models for CMV infections in humans.

\section{RCMV replication in salivary gland cells}

The salivary gland is the preferred organ for CMV infections. RCMV replication in salivary gland cells is detectable approximately 8 days after infection, whereas during the first week after infection, infectious virus is predominantly produced in internal organs, such as liver and spleen $(75,77,497)$. Infection of salivary glands is characterized by a long term production of high amounts of infectious virus. The salivary gland consists of three major glands, designated the submandibular, parotid and sublingual gland. Although some data were available about RCMV replication in the submandibular gland, little was known about virus replication in the parotid and the sublingual salivary gland. Replication of RCMV in the major salivary glands is described in more detail in chapter 2. Assessment of viral load in the submandibular, parotid and sublingual gland of young adult animals, revealed that the submandibular gland was the preferred organ for RCMV replication. In contrast, in newborn rats, the main site of viral replication was the sublingual gland. It is possible that the maturation process of the sublingual gland enables optimal RCMV replication shortly after birth, whereas this permissiveness for the virus is lost when the maturation process is completed. Interestingly, it was shown by immunohistochemistry, in situ hybridization and electron microscopy, that, in all infected animals, viral replication was restricted to striated duct cells. This is in sharp contrast to MCMV where replication is exclusively found in acinar cells. Differences in cell tropism between RCMV and MCMV are especially fascinating since it is known that both viruses possess a lot of similarities and the genome of RCMV and MCMV show the highest homology of all known CMVs. Infection of rats resulted in a cellular inflammatory response, which was predominantly located in the interlobular duct region. Only a few inflammatory cells were found in the neighborhood of infected striated duct cells. A similar observation was made in the murine system (216). The inability of inflammatory cells to reach infected striated duct cells may be one of the explanations for the persistence of CMV in the salivary glands.

It was shown that infectious virus was produced in large cytoplasmic vesicles and is excreted into the lumen of the salivary gland by apical cell budding of the vesicle. The chronic production of high amounts of infectious virus could have other consequences, rather than solely providing infectious virus for horizontal transmission. Shedding of virus from the salivary gland may result in re-infection of the host. In order to study the biological significance of RCMV replication in the salivary gland cells, we employed a model in which the salivary glands were removed in a subset of rats prior to infection (chapter 3). 
Surgically desalivated rats were compared to sham-operated animals after a lethal and sublethal infection with RCMV, respectively. Survival was recorded and presence of infectious virus was determined by plaque assay and the presence of viral DNA was assessed by PCR. We demonstrated that desalivation did not have an effect on the clinical course of RCMV disease and on RCMV-induced mortality, but a significantly higher titer of anti-RCMV antibodies was found in sham-operated rats than in desalivated animals during long-term follow-up. These data indicate that shedding of virus or viral antigens to the systemic circulation occurs from the salivary gland via virus-containing saliva and that this re-introduction of virus or its components is responsible for boosting the humoral response, resulting in high antibody titers. Interestingly, we were unable to detect viral DNA and infectious virus in spleen, liver and lung of infected animals at 1 year p.i., but as expected, the submandibular glands still yielded high amounts of infectious virus. These data suggest that boosting of the humoral response is caused by shedding of viral antigens from the salivary glands and that infectious virus shed from the salivary gland is neutralized by already existing circulating anti-RCMV antibodies, a phenomenon described previously (250).

\section{The $\mathbf{r 1 4 4}$ gene product interferes with the innate immune system}

$\mathrm{CMV}$ infection is the most common congenital infection in humans. Approximately $5 \%$ of infected newborns are born with CMV disease $(128,393)$. Prognosis in this group is poor and the clinical presentation during follow-up is mainly characterized by neurological deficits (106, 387). A significant proportion of the asymptomatic infected newborns $(5 \%)$ will suffer from long term sequelae of which sensorineural hearing loss is most common (166, 167). Sensorineural deterioration due to asymptomatic infection usually progresses undetected and it has been reported that screening of these patients fails in over $50 \%$ of the cases (166). In contrast to the human situation, RCMV is not able to cross the placental barrier (unpublished results). It is therefore likely that vertical transmission of virus in rats is of little importance and that newborn rats become infected after birth. Thus, an experimental rat model for congenital infection in humans can only be achieved by infecting newborn rats shortly after birth. Experimental RCMV infection in newborn rats is described in chapter 4. RCMV infection of newborn rats resulted in similar distribution of virus during the course of infection as did infection of immunocompromised adult rats. RCMV was present in liver, spleen and lung at 3 and 5 days p.i., whereas RCMV could only be detected in the salivary glands at 21 days p.i. Furthermore, RCMV infection resulted in an inflammatory response in infected organs, which consisted of monocytes/macrophages, T cells and NK cells. The immune system of neonatal rats was able to control CMV infection to some extent, since animals did not show symptoms of CMV disease and did survive for at least 21 days after inoculation of $10^{4} \mathrm{PFU}$ of virus. In 
contrast, it is known that survival rates are greatly impaired when rats of 4 weeks old are subjected to 5 Gy of TBI prior to injection of $10^{5} \mathrm{PFU}$ of RCMV. The usage of TBI as immune suppressive regimen affects the number of granulocytes, NK cells, monocytes, $B$ cells and $\mathrm{T}(\mathrm{CD} 4+$ and $\mathrm{CD} 8+$ ) cells in peripheral blood of irradiated animals significantly and leads to a transient state of severe immunosuppression (525). Peripheral white blood cells are suppressed during the acute phase of infection and the number of cells return to control levels at approximately 3-4 weeks after irradiation (525). The natural, unaffected immune system of neonatal rats enables disseminated RCMV infection, but prevents uncontrolled viral replication and CMV-induced multi-organ failure. This optimal equilibrium between on the one hand the level of RCMV replication and on the other hand the control of RCMV infection by immune effector functions in neonatal rats enables us to study the interaction of the virus with the immune system of the host.

CMV has developed several mechanisms to evade immune surveillance (353). One of those mechanisms is to downregulate cellular MHC class I expression. However, according to the 'missing self hypothesis', downregulation of MHC class I renders a cell susceptible to NK cell killing (316). Viral encoded MHC class I homologs have been identified on the genome of human CMV (UL18, (39)), MCMV (m144, (155)) and RCMV $\left(\mathrm{r} 144,{ }^{(43)}\right)$.

An RCMV strain in which the r144 gene is disrupted was generated in our laboratory (43) and infection of immunocompromised adult rats with this deletion mutant showed that there were no significant differences in tissue distribution and virus titers between wt RCMV- and RCMV $\Delta r 144$-infected animals (43). Since it is thought that the r144 gene might play a role in virus-induced evasion of immune surveillance, it is possible that the high level of irradiation-induced immunosuppression was responsible for those results. In order to study the role of r144 in the pathogenesis of RCMV infection in rats with a normal unaffected immune system, neonatal rats were challenged with either wt RCMV or RCMVAr144 and the presence of infectious virus as well as viral DNA was assessed in various organs at different time points p.i. Replication of RCMV $\Delta r 144$ in the spleen and the salivary gland was severely restricted compared to wt RCMV at 3 and 21 days p.i., respectively. RCMVAr144, has previously been shown to have similar replication characteristics as wt virus in vitro as well as in vivo ${ }^{(43)}$, suggesting that RCMVAr144 replicates as efficiently as wt RCMV in the absence of a fully functional immune system. In addition, also a significant lower number of monocytes/macrophages and NK cells were found in spleens of RCMVAr144-infected rats than in those of wt RCMV-infected animals at 3 days p.i. These results are in concert with previous results, using a local infection model, in which either RCMV $\triangle$ r144 or wt RCMV were subcutaneously injected in the hind paw of immunocompromised adult rats. In this model, both viruses were shown to replicate with similar efficiency locally in the hind paw. However, a significantly lower number of 
monocytes/ macrophages was found at the site virus injection. In this case, however, a similar number of NK cells was found in the rat hind paw of RCMV $\Delta$ r144- than in those of wt RCMV-infected animals. These data from the neonatal- and rat hind paw model are in concert with previous reports obtained from the human (116, 306) and murine system (282). The UL18 gene product was shown to interact with a membrane receptor, designated LIR1 , which is predominantly expressed on monocytes and B lymphocytes, but only on a minor subset of NK cells ${ }^{(114)}$. Cretney et al. showed that the $\mathrm{m} 144$ gene product provided some protection against NK cell-mediated killing of lymphoma cells transfected with m144 in vitro, but that in vivo, the major effect of $\mathrm{m} 144$ is to regulate NK cell accumulation and activation (116). These data show that the CMV-encoded MHC class I homolog is more likely to compromise the innate immune system as a whole, rather than to solely inhibit NK cell-mediated killing.

\section{RCMV infection of renal allografts results in an increased and prolonged expression of adhesion molecules.}

Current immunosuppressive regimens and anti-rejection therapies prevent renal allograft failure due to acute rejection in the majority of cases. However, several years after transplantation, a significant number of renal allografts are lost as a result of chronic rejection. The level of histoincompatibility, inadequate immunesuppression, acute rejection and viral infections, especially CMV infections, have been suggested as risk factors for chronic rejection $(14,38,209,390)$. Although it is generally accepted that CMV infection plays a role in the acceleration of chronic rejection $(20,193,277,281,292,339,380)$ the mechanism involved is unclear. To study the role of CMV infection chronic renal allograft rejection, the rat model developed by Soots et al. ${ }^{(483)}$ was used.

Chapter $\mathbf{5}$ focuses on the accelerated development of chronic rejection in RCMV infected rats receiving a kidney allograft under triple drug immunosuppression. It was shown previously that renal allografts harvested from rats receiving triple drug immunosuppression showed histological signs of chronic rejection at 40-60 days after transplantation (483). The histological diagnosis of chronic rejection includes perivascular and interstitial inflammation, fibrosis, glomerulosclerosis, vascular intimal thickening and tubular atrophy $(391,480)$. The peak of inflammation in these allografts was seen 5-10 days p.i. and was associated with lymphoid activation and induction of adhesion molecules (259). Thereafter, renal inflammation gradually decreases while the histological characteristics of chronic rejection became more and more prominent. RCMV antigens could be detected in endothelial cells of renal allografts at 7 days p.i. (294). Strikingly, RCMV infection increased and prolonged inflammation and accelerated the development of chronic rejection significantly. The histological criteria for chronic rejection were already fulfilled before 20 days after transplantation (294). RCMV infection of renal allografts was associated with an 
increased and prolonged expression of adhesion molecules ICAM-1 and VCAM-1 on endothelial cells and their ligand molecules, LFA-1 and VLA-4, expressed on leukocytes. Expression of adhesion molecules is mediated via cytokines, such as IL-1, TNF-alpha and IFN-gamma, which are produced by activated lymphocytes. On the other hand, CMV has been shown to upregulate IL-1 $\beta$ gene expression, which may lead to production of IL- 1 by mononuclear cells (235). In addition, IE genes of CMV have been found to upregulate IL-2 and IL-2R genes (179). Furthermore, CMV induces TNF-alpha in monocytes/macrophages (472), as well as in vivo (202). RCMV mediated production of cytokines may result in a generalized increased and prolonged expression of adhesion molecules, even on uninfected endothelial cells of the graft (541). These data suggest that RCMV influences the induction phase of the alloresponse and determines the magnitude of this process from that point onward.

\section{Treatment of CMV infections in immunocompromised rats.}

Despite the drastic decrease in CMV related morbidity since the introduction of HAART, CMV-induced encephalomeningitis and its treatment are still a major clinical problem in patients with end-stage HIV infection. The effect of several antiviral compounds on RCMV-induced encephalomeningitis in immunocompromised rats is described in chapter 6. Rats were intracerebrally infected and were systemically treated with either HPMPC, DHPG, HIS, or DHPG in combination with HIS. After intra-cerebral infection, the viral antigens could be detected in mononuclear cells in the meninges and endothelial cells of small vessels. In addition, an inflammatory response consisting of mononuclear cells and $T$ cells and scattered foci of necrosis harboring RCMV antigens could be demonstrated in the meninges and to a lesser extent in the brain. RCMV infection of endothelial cells is associated with a local inflammatory response, in a similar manner as described previously in the rat hind paw model (399). The observed histological alterations in our rat model closely resemble the pathology of CMV-induced meningoencephalitis in AIDS patients $(208,360)$. Viral replication in endothelial cells may be a portal for entry of CMV into the central nervous system. Treatment with a single dose of $20 \mathrm{mg} / \mathrm{kg}$ HPMPC significantly reduced the amount of infectious virus in brain and spleen compared to all other treatment modalities, which were administered daily at the appropriate doses for 5 days. One explanation for this therapeutic failure in the model used could be poor penetration of the antiviral compounds into the brain area, which permits initial replication cycles of the virus.

Tissue damage due to CMV infection is at one hand caused by direct virus-induced cell damage and on the other hand mediated by the mounted immune response. For example, the HAART-associated reconstruction of immune effector functions in AIDS patients renders patients with a history of CMV retinitis susceptible to IRV, a condition 
characterised by retinal inflammation without any signs of CMV relapse (256, 257). In addition, CMV pneumonitis in AIDS patients is rare and follows a mild course, whereas CMV pneumonitis in BMT is a frequent and lethal condition (37). This suggest that the inflammatory immune response after CMV infection plays an important role in causing tissue dysfunction. Drugs with anti-CMV and anti-inflammatory properties may be beneficial. Chapter 7 describes the evaluation of 2 compounds, DFO and DTPA, which are thought to inhibit viral replication and decrease oxidative stress. The mechanism by which both compounds act is not fully understood, but it is thought that the inhibition of an iron-dependent ribonucleotide reductase may be responsible for the inhibition of RCMV replication. The antiviral effect of both compounds was assessed in vitro as well as in vivo, using a generalized and a local infection model. In concert with previous results $(103,105)$, DFO and DTPA exhibited an antiviral effect in vitro, although DTPA was more potent than DFO. In immunocompromised rats, DFO and DTPA were unable to prevent RCMV mortality and RCMV disease. Neither DFO nor DTPA reduced virus replication in organs significantly after a sublethal RCMV challenge. A local infection model, in which RCMV was injected in the rat hind paw of immunocompromised animals, enabled us to study the effect of DFO and DTPA on the spread of virus from the infection site as well as the effect of both drugs on the local inflammatory response. Treatment with DFO or DTPA was unable to reduce the swelling of the hind paw, did not affect the viral load as measured by the number of RCMV antigens expressed in rat hind paw tissue and failed to diminish the number of infiltrating leukocytes. However an antiviral effect was observed in the local infection model, as shown by a significant decrease in the number of rats harboring infectious virus in spleen and liver after treatment with DFO or DTPA. The in vivo transplantation study using rat liver allografts by Martelius and co-workers (331) supported this finding. In their study an inhibitoy effect of metal chelators on CMV replication, inflammation an on bile-duct damage was detected. In addition, DFO has been shown to reduce auto-immune-mediated retinal inflammation (252) and iatrogeneic inflammation of the rat hind paw (53) significantly. Both compounds were shown to inhibit 1) mitogen- and allogen- induced proliferation of peripheral blood lymphocytes, 2) NK cell function and 3) adhesion molecule expression (450). More importantly, in a case report, administration of DFO to an AIDS patient suffering from CMV retinitis, refractory to ganciclovir and foscarnet, inhibited progression of ocular changes. These data indicate that metal chelators as DFO and DTPA have to some extent antiviral capacities in vivo, but that these compounds as reported by Martelius et al. are especially usefull in settings with evident immune activation, such as organ transplantation recipients. 


\section{Concluding remarks}

In this thesis several aspects of CMV infection and CMV disease have been studied in a rat model. The model used in this thesis gave us the opportunity to study the replication of the virus and the pathology it induced in more details. Although, RCMV infections closely resemble CMV infections in man, one should bear in mind that translation of data from CMV-infected laboratory animals to the human situation should be made with care, even when the genome of both strains shows a high level of homology. Nevertheless, the ability to control most variables in an experimental setting using animals and the appropriate viruses enable us to unravel mechanisms of CMV disease. Data from these models may provide new potential targets for antiviral treatment. 


\section{Samenvatting}

De seroprevalentie van CMV is hoog in de gezonde populatie. Een eerste infectie met CMV is normaliter asymptomatisch. In een minderheid van de gevallen kan een griepachtig beeld ontstaan. Levensbedreigende ziekte door CMV infectie bij gezonde personen komt voor, maar is uiterst zeldzaam. Patiënten met een immuundeficiëntie, zoals AIDS- of transplantatiepatiënten, lopen risico op ernstige CMV ziekte door een primaire infectie of reactivatie van latent aanwezig CMV. Ganciclovir, cidofovir en foscarnet zijn middelen die gebruikt worden voor de behandeling van ziekte door CMV infectie.

Ongeveer 20 jaar geleden werd uit ratten CMV geïsoleerd (RCMV). Het RCMV model wordt gebruikt voor onderzoek naar pathogenese en het testen van nieuwe potentieel antivirale middelen. In dit model wordt het virus meestal intraperitoneaal toegediend, maar ook subcutane en intraveneuze toediening kunnen gebruikt worden. $\mathrm{Na}$ intraperitoneale toediening wordt het virus in de eerste week na infectie voornamelijk gevonden in abdominale en thoracale organen zoals lever, milt, nieren en longen. Na de eerste week kan geen infectieus virus in deze organen meer worden aangetoond. Wel blijft het DNA van CMV in deze organen aantoonbaar (latentie). Vanaf ongeveer 8 dagen na infectie wordt er infectieus virus aangetroffen in de speekselklieren. De productie van infectieus virus houdt lange tijd (enkele maanden) aan. Hoofdstuk 2 beschrijft de replicatie van RCMV in de speekselklieren van de rat. Met in situ hybridisatie, electronen microscopie en immunohistochemie is gebleken dat, ondanks verschillen in speekselkliertropisme tussen pasgeboren- en jongvolwassen ratten, uitsluitend striatale cellen geïnfecteerd zijn. Het virus wordt met het speeksel uitgescheiden. Dit is een van de mechanismen waarmee het virus zich kan verspreiden tussen soortgenoten. Het is onduidelijk of het uitscheiden van speeksel met CMV een re-infectie veroorzaakt. In hoodstuk 3 worden ratten met en zonder speekselklieren geïnfecteerd met RCMV om het infectieverloop in beide groepen te vergelijken. Het acute infectieverloop na een letale dosis RCMV verschilt niet in ratten met en zonder speekselklieren. In een tweede experiment, waarbij ratten gedurende een jaar gevolgd werden, werd een verschil in antilichaamtiter gevonden. Ratten zonder speekselklieren hadden lagere antilichaamtiters na infectie dan ratten met speekselklieren. Dit suggereert dat uitscheiding van het virus of virale antigenen plaatsvindt via speekselklieren en dat dit mede verantwoordelijk is voor activatie van de humorale immuunrespons.

In tegenstelling tot jongvolwassen ratten heeft men bij pasgeboren ratten geen algehele lichaamsbestraling nodig om een ernstig verlopende RCMV infectie te bewerkstelligen. Hoewel het immuunsysteem van pasgeboren ratten nog onrijp is, is het echter in staat om de infectie uiteindelijk het hoofd te bieden en overlijden te voorkomen. Het gebruik van pasgeboren ratten is dus een uitermate geschikt model voor het bestuderen van de interactie van het virus met het immuunsysteem. Eerder onderzoek 
wijst erop dat een aantal genen in het CMV genoom coderen voor eiwitten die interfereren met het immuunsysteem. Door het immuunsysteem te misleiden en een geïnfecteerde cel als "gezond" te presenteren kan de virusgeïnfecteerde cel ontsnappen aan het immuunsysteem en er derhalve voor zorgen dat het virus overleeft. De door CMV gecodeerde MHC klasse I homoloog is geassocieerd met het proces van immuun evasie. Het cellulaire MHC klasse I eiwit wordt tot expressie gebracht op de celmembraan van elke kernhoudende lichaamscel en speelt een cruciale rol in de afweer tegen virusinfecties. In hoofdstuk 4 wordt de bijdrage van de virale gecodeerde MHC klasse I homoloog aan de pathogenese nader bestudeerd door virussen met (wt RCMV) en zonder de MHC klasse I homoloog (RCMV $\Delta$ r144) met elkaar te vergelijken. Hiervoor werden pasgeboren ratten intraperitoneaal geïnfecteerd met een van beide virussen. Eerder onderzoek liet zien dat beide virussen in vitro even efficiënt repliceerden. Daarnaast konden er geen verschillen in virusconcentratie worden aangetoond tussen wt RCMV- en RCMV $\Delta$ r144-geïnfecteerde immuundeficiënte ratten. In deze experimenten werd de virale replicatie niet beïnvloed door een aanwezig immuunsysteem. Echter in het RCMVinfectiemodel met pasgeboren ratten wordt er een significant lagere hoeveelheid infectieus virus gevonden in de milt en speekselklieren van RCMV $\Delta \mathrm{r} 144-$ dan in milt en speekselklieren van wt RCMV-geïnfecteerde ratten. Deze resultaten in de verschillende modellen suggereren dat het verschil in virusreplicatie tussen beide virussen veroorzaakt wordt door de activiteit van het immuunsysteem. De functie van de virale MHC klasse I homoloog is echter nog onduidelijk. Er zijn aanwijzingen dat de viraal gecodeerde MHC klasse I homoloog, naast zijn functie als vervanger van het cellulaire MHC klasse I, ook een interactie aangaat met cellen van het niet-specifieke immuunsysteem.

CMV speelt een belangrijke rol bij orgaantransplantaties. De mate van histoincompatibiliteit, inadequate immunosuppressie, episodes van acute afstoting en virale infecties (waaronder CMV) worden geassocieerd met de chronische afstotingsreactie. Hoewel het verband tussen CMV infectie en chronische afstoting algemeen geaccepteerd is, is het achterliggende mechanisme onduidelijk. Uit eerder onderzoek in het RCMV model is gebleken dat RCMV infectie de chronische afstotingsreactie van een allogeen niertransplantaat versnelt. De versnelde afstotingsreactie door RCMV gaat gepaard met verhoogde expressie van de adhesiemoleculen ICAM-1 en VCAM-1 op endotheelcellen van de getransplanteerde nier en hun ligand moleulen LFA-1 en VLA-4 op de aanwezige leukocyten (Hoofdstuk 5). Het is bekend dat CMV de expressie van sommige cytokines, zoals IL-1 $\beta$, IL-2, IL-2R en TNF- $\alpha$, kan verhogen. De langdurig verhoogde expressie van adhesiemoleculen op geïnfecteerde en niet-geïnfecteerde endotheelcellen kan verklaard worden door de verhoogde cytokine productie. Adhesiemolecuul expressie is essentieel voor het uittreden van inflammatoire cellen uit de bloedbaan en staat als dusdanig aan de basis van de afstotingsreactie van de getransplanteerde nier. 
De behandeling van ziekte door CMV infectie is complex. Aangezien CMV infectie voomamelijk een probleem is in immuundeficiente patienten zou theoretisch de beste behandeling het opheffen van de immuundeficièntie zijn. Een voorbeeld hiervan betreft het behandelen van AIDS patienten met HAART. Door de replicatie van HIV te reduceren zal het immuunsysteem ( $T$-helper cellen) van de patiènt tijdelijk kunnen herstellen. De incidentie en de ernst van CMV infecties in deze populatie daalt dan drastisch. In een aantal patiênten wordt echter een immunologisch gemedieerde orgaanschade gemeld. Dit treedt voornamelijk op ter hoogte van de retina. Het immuunsysteem gaat dan massaal het geinfecteerde weefsel te lijf en schiet hierbij zijn doel voorbij waardoor zogenaamde immuunpathologische schade optreedt. In hoofdstuk 6 wordt de werking van enkele antivirale middelen bestudeerd. Deze middelen remmen direct de virale replicatie. Om dit te onderzoeken werden immuundeficięnte ratten intracerebraal geilnfecteerd met RCMV en vervolgens behandeld met HPMPC (cidofovir), DHPG (ganciclovir), Hyper Immuun Serum (HIS) en een combinatie van HIS en DHPG. In het beschreven ratmodel kwam het histopathologisch beeld van de hersenen overeen met de pathologie zoals die gezien wordt in CMV-geïnduceerde meningo-encephalitis in AIDS patiènten. Behandeling met HPMPC was, met betrekking tot inhibitie van virale replicatie, superieur aan de andere geteste antivirale middelen. Daarbij werd HPMPC slechts eenmalig gegeven, terwijl DHPG gedurende 5 dagen dagelijks toegediend werd. In hoofdstuk 7 worden de resultaten beschreven van experimenten waarbij middelen getest worden waarvan naast een directe antivirale werking, ook een immuunmodulerende werking worden toegeschreven. DFO en DTPA zijn metaalchelatoren en hun antivirale werking wordt toegeschreven aan inhibitie van een ijzerafhankelijk ribonucleotide reductase. Gebruik van deze middelen in RCMVgeïnfecteerde ratten toont aan dat het antivirale effect van beide middelen in ratten gering is: DFO en DTPA konden overlijden ten gevolge van een letale dosis CMV niet voorkomen. Ook waren beide middelen niet in staat om de concentratie van infectieus virus in organen van niet-letaal geïnfecteerde ratten te verminderen. Wel werd er een beperkt antiviraal effect gezien van beide middelen in een lokaal infectiemodel. In dit model wordt virus subcutaan in de achterpoot van een immuundeficiënte rat gespoten. Infectieus virus wordt nu geleidelijk aan de lichaamscirculatie afgegeven. Behandeling met DFO en DTPA resulteerde in een significante vermindering van het aantal dieren met geïnfecteerde interne organen zoals lever en milt in vergelijking met controle dieren die geen behandeling kregen. DFO en DTPA hebben dus een beperkte waarde als antiviraal middel. Deze resultaten staan echter in schril contrast met de resultaten die door de groep van Lautenschlager verkregen werden in een allogeen levertransplantatiemodel. DFO bleek in dit model de afstotingsreactie en de virale replicatie te verminderen. Dit suggereert dat metaalchelatoren geïndiceerd kunnen zijn in patiënten met een duidelijke immuunactivatie. Verder onderzoek naar de werking van metaalchelatoren in 
transplantatiemodellen is geïndiceerd.

In dit proefschrift worden enkele aspecten van CMV infectie en CMV ziekte bestudeerd in een ratmodel. De ratmodellen die in dit proefschrift gebruikt werden, gaven ons de mogelijkheid om de virale replicatie en de pathologie die het induceert te bestuderen. Hoewel ziekte door RCMV en CMV niet veel van elkaar verschillen, is echter voorzichtigheid geboden bij het extrapoleren van gegevens uit het RCMV model naar de humane situatie. Het voordeel van diermodellen is de mogelijkheid om veel variabelen te controleren en systematisch de mechanismen van CMV ziekte te ontrafelen. 


\section{List of publications}

Kloover, J.S., A. E. van den Bogaard, J.G. avn Dam, M.J. Blok, G. Grauls, C. Vink, C.A. Bruggeman. Persistent rat cytomegalovirus (RCMV) infection of the salivary glands contributes to the anti-RCMV antibody response in infected rats. Submitted.

Kloover, J.S., G. Grauls, M.J. Blok, C. Vink, C. A. Bruggeman. 2002 A rat cytomegalovirus strain with a disruption of the $\mathrm{r} 144 \mathrm{MHC}$ class I-like gene is attenuated in the acute phase of infection in neonatal rats. Arch. Virol. 147: 813-824.

Beisser, P. S., J. S. Kloover, G. E. Grauls, M. J. Blok, C. A. Bruggeman and C. Vink. 2000. The r144 major histocompatibility complex class I-like gene of rat cytomegalovirus is dispensable for both acute and long-term infection in the immunocompromised host. $J$ Virol. 74:1045-1050.

Kloover, J. S., J. L. Hillebrands, G. de Wit, G. Grauls, J. Rozing, C. A. Bruggeman and P. Nieuwenhuis. 2000. Rat cytomegalovirus replication in the salivary glands is exclusively confined to striated duct cells. Virchows Arch. 437:413-421.

Kloover, J. S., M. Scholz, J. Cinatl, Jr., I. Lautenschlager, G. E. Grauls and C. A. Bruggeman. 1999. Effect of desferioxamine (DFO) and calcium trinatrium diethylenetriaminepentaacetic acid (DTPA) on rat cytomegalovirus replication in vitro and in vivo. Antiviral Res. 44:55-65.

Kloover, J. S., A. P. Soots, L. A. Krogerus, H. O. Kauppinen, R. J. Loginov, K. L. Holma, C. A. Bruggeman, P. J. Ahonen and I. T. Lautenschlager. 2000. Rat cytomegalovirus infection in kidney allograft recipients is associated with increased expression of intracellular adhesion molecule-1 vascular adhesion molecule-1 and their ligands leukocyte function antigen-1 and very late antigen-4 in the graft. Transplantation. 69:2641-7.

Kloover, J. S., W. Y. Vanagt, F. S. Stals and C. A. Bruggeman. 1997. Effective treatment of experimental cytomegalovirus-induced encephalo-meningitis in immunocompromised rats with HPMPC. Antiviral Res. 35:105-12. 
Lautenschlager, I., A. Soots, L. Krogerus, K. Inkinen, J. Kloover, R. Loginov, K. Holma, H. Kauppinen, C. Bruggeman and J. Ahonen. 1999. Time-related effects of cytomegalovirus infection on the development of chronic renal allograft rejection in a rat model. Intervirology. 42:279-84.

Stals, F. S., S. S. Wagenaar, J. S. Kloover, W. Y. Vanagt and C. A. Bruggeman. 1996. Combinations of ganciclovir and antibody for experimental CMV infections. Antiviral Res. 29:61-4 


\section{Dankwoord / acknowledgements}

Toen ik als eerste jaars student met met Ward Vanagt via Frans Stals bij de afdeling Medische Microbiologie geîntroduceerd werd, besefte ik niet dat dit snuffelen aan het wetenschappelijk onderzoek zulke gevolgen kon hebben. Gaande weg heb ik op deze afdeling veel mensen beter leren kennen die ik nu gelukkig tot mijn vriendenkring mag rekenen. Het tot stand komen van dit proefschrift was niet mogelijk geweest zonder de medewerking en het heerlijke werkklimaat van deze afdeling. Een aantal mensen verdienen speciale aandacht:

Allereerst natuurlijk mijn promotor Cathrien Bruggeman. Beste Cathrien, zoals je je misschien nog wel kunt herinneren heeft het even geduurd voordat ik je bij je voornaam durfde noemen. Ik bewonder je betrokkenheid, je interesse voor de mensen op je afdeling en het feit dat je niet bang bent om nieuwelingen verantwoordelijkheden te geven. $1 \mathrm{k}$ wil je bedanken voor de mogelijkheid om bij jou promotieonderzoek te mogen doen. Ik kijk terug op een heerlijke "MMB-tijd".

Beste Gert, als "moeder overste" van het in vivo-research lab heb je me mijn eerste stapjes op het lab laten zetten. Je praktische kennis over protocollen en je bijna onfeilbare geheugen van al eerder uitgevoerde proeven zijn legendarisch. Daarbij schepte je een uiterst ontspannen werksfeer waarin bijna alles mogelijk was. Er zijn trouwens maar weinig vrouwen die mij aan mijn oren hebben meegenomen om mij op mijn 'fouten' (lees rommel) te wijzen. Ik wil je bedanken voor de fijne samenwerking en ik ben zeer benieuwd hoe je tulpenjurk gaat staan!

Beste Kees, hoewel we niet dagelijks met elkaar te maken hadden heb je toch een belangrijke rol gespeeld bij het tot stand komen van dit proefschrift. Ik wil je bedanken voor de discussies over en je correcties van mijn artikelen. Speciaal wil ik je bedanken voor de hulp bij het tot stand komen van de laatste twee publicaties.

Een groot gedeelte van mijn onderzoek liep simultaan met dat van Joanne van Dam. Beste Joanne, ondanks dat onze insteek van onderzoek verschilde, had ons "in vivo werk" veel raakvlakken. Ik wil je bedanken voor alle hulp die ik van je gekregen heb. Als er iemand onbaatzuchtig zijn hulp aanbiedt dan ben jij het wel.

Mijn kamergenoten, Rien Blok, Suzanne Kaptein, Erik Beuken, Coen van Kleef, Yvonne Gruijthuijzen, Patrick Beisser, Roosje Beisser-van Gorp (vreemde eend in de bijt), Frank Stassen, Inge Vliegen en Geoffrey Kloppenburg voor een (veel te) gezellige tijd op de kamers. Discussies over alles en nog wat in combinatie met veel chocola en snoepgoed waren aan de orde van de dag. Bedankt iedereen voor alle collegialiteit en vriendschap! Ik wil Suzanne en Roosje bedanken voor de heerlijke wandeltochten in het schone Limburgse landschap.

Verder gaat mijn dank uit naar Martine Hulsbosch, Bieke Vanherle, Monique Coomans, 
Magdalena Garcia en Sabine Stevens voor de praktische ondersteuning met betrekking tot de PCRs en het inbedden, snijden en kleuren van coupes.

Frans Stals wil ik bedanken voor zijn enorme enthousiasme ten aanzien van het wetenschappelijk onderzoek. Zoals je ziet heeft het aanstekelijk gewerkt!

In het hoge noorden huist de Vakgroep Histologie en Celbiologie van de Universiteit Groningen onder leiding van prof. dr. Paul Nieuwenhuis. Ik wil prof. Nieuwenhuis, JanLuuk Hillebrands en de gehele afdeling bedanken voor de fijne samenwerking. Beste JanLuuk, bedankt voor je mateloze enthousiasme en gastvrijheid! We hebben in ieder geval 'explosief' onderzoek verricht. Hoofdstuk 2 was verder niet mogelijk geweest zonder de afdeling Cel biologie en Elektron Microscopie van de Universiteit Groningen. Speciale dank gaat uit naar Guus de Wit die veel tijd in de EM analyses heeft gestoken.

I would like to thank dr. Irmeli Lautenschlager from the University Hospital in Helsinki for the opportunity to visit her wonderful country and to participate in her research group. My special regards go to Timi Martelius and his wife Laura. Thank you for your friendship and hospitality! I would also like to thank Raisa Loginov for her excellent technical support.

Verder gaat mijn dank uit naar mijn ouders en zus. Lieve pa, ma en Annemieke, bedankt voor jullie geduld bij het luisteren naar mijn "spannende" verhalen over ratten, virussen en pipetten. Jullie zijn een geweldige steun voor mij geweest. $\mathrm{Pa}$, bedankt voor je bemoeienissen met de Sherlock Holmes op de voorkant!

Lieve Susan, het belangrijkste gedeelte van mijn dankwoord is gereserveerd voor jou. lk wil je bedanken voor je eindeloze steun, toewijding en liefde. Ik hoop dat ik hetzelfde voor jou kan betekenen als jij jouw onderzoek gaat afronden. Ik ben trots op je en ik weet zeker dat je het fantastisch gaat doen! 


\section{Curriculum vitae}

Jeroen Steven Kloover werd geboren op 19 december 1975 te Rotterdam. Na het behalen van zijn WWO eindexamen in 1994 aan de CSG Blaise Pascal te Spijkenisse, startte hij in dat zelfde jaar met de studie geneeskunde aan de Faculteit der Geneeskune van de Universiteit van Maastricht. Na het propedeutisch examen in 1995 volgde het docteraal examen in 1998. Tijdens zijn studie raakte hij als student betrokken bij het wetenschappelijk onderzoek van de afdeling Medische Microbiologie van het Academisch ziekenhuis Maastricht (AzM). Aanvankelijk was de begeleiding in handen van Dr. F. Stals en later van prof. dr. C.A. Bruggeman. Dit onderzoek resulteerde in promotieonderzoek met prof. dr. C.A. Bruggeman als promotor. Van 1998 tot en met 2001 volgde een periode van co-schappen in het Academisch ziekenhuis Maastricht. In 2001 werd het arts-examen behaald. Vanaf mei 2001 werkt hij als arts-assistent niet in opleiding op de afdeling longziekten van het Erasmus Medisch Centrum Rotterdam onder prof. dr. H. Hoogsteden. Per september 2002 start hij aan de Universiteit van Utrecht met de opleiding tot huisarts. 


\section{References}

1. 1995. Intravenous versus oral ganciclovir: European/Australian comparative study of efficacy and safety in the prevention of cytomegalovirus retinitis recurrence in patients with AIDS. The Oral Ganciclovir European and Australian Cooperative Study Group. Aids. 9:471-7.

2. 1997. Parenteral cidofovir for cytomegalovirus retinitis in patients with AIDS: the HPMPC peripheral cytomegalovirus retinitis trial. A randomized, controlled trial. Studies of Ocular complications of AIDS Research Group in Collaboration with the AIDS Clinical Trials Group. Ann Intern Med. 126:264-74.

3. Adam, E., J. L. Melnick, J. L. Probtsfield, B. L. Petrie, J. Burek, K. R. Bailey, C. H. McCollum, and M. E. DeBakey. 1987. High levels of cytomegalovirus antibody in patients requiring vascular surgery for atherosclerosis. Lancet. $2: 291-3$.

4. Adler, S. P. 1989. Cytomegalovirus and child day care. Evidence for an increased infection rate among day-care workers. N Engl J Med. 321:1290-6.

5. Adler, S. P. 1986. Cytomegalovirus infection in parents of children at day-care centers. N Engl J Med. 315:1164-5.

6. Adler, S. P. 1988. Cytomegalovirus transmission among children in day care, their mothers and caretakers. Pediatr Infect Dis J. 7:279-85.

7. Adler, S. P., J. K. Hur, J. B. Wang, and G. W. Vetrovec. 1998. Prior infection with cytomegalovirus is not a major risk factor for angiographically demonstrated coronary artery atherosclerosis. J Infect Dis. 177:209-12.

8. Ahlfors, K., S. A. Ivarsson, and S. Harris. 1999. Report on a long-term study of maternal and congenital cytomegalovirus infection in Sweden. Review of prospective studies available in the literature. Scand J Infect Dis. 31:443-57.

9. Ahn, K., A. Angulo, P. Ghazal, P.. A. Peterson, Y. Yang, and K. Fruh. 1996. Human cytomegalovirus inhibits antigen presentation by a sequential multistep process. Proc Natl Acad Sci U S A. 93:10990-5.

10. Ahn, K., A. Gruhler, B. Galocha, T. R. Jones, E. J. Wiertz, H. L. Ploegh, P. A. Peterson, Y. Yang, and K. Fruh. 1997. The ER-luminal domain of the HCMV glycoprotein US6 inhibits peptide translocation by TAP. Immunity. 6:613-21.

11. Alberola, J., V. Dominguez, L. Cardenoso, J. Lopez-Aldeguer, M. Blanes, F. Estelles, C. Ricart, A. Pastor, R. Igual, and D. Navarro. 1998. Antibody response to human cytomegalovirus (HCMV) glycoprotein B (gB) in AIDS patients with HCMV end-organ disease. J Med Virol. 55:272-80. 
12. Alberola, J., A. Tamarit, R. Igual, and D. Navarro. 2000. Early neutralizing and glycoprotein B (gB)-specific antibody responses to human cytomegalovirus (HCMV) in immunocompetent individuals with distinct clinical presentations of primary HCMV infection. J Clin Virol. 16:113-22.

13. Alberola, J., A. Tamarit, and D. Navarro. 1999. Functional antibody response to human cytomegalovirus in immunocompetent and HIV-1 infected individuals with antibodies that inhibit virus penetration into cells and intercellular transmission of viral infection. J Med Microbiol. 48:947-954.

14. Almond, P. S., A. Matas, K. Gillingham, D. L. Dunn, W. D. Payne, P. Gores, R. Gruessner, and J. S. Najarian. 1993. Risk factors for chronic rejection in renal allograft recipients. Transplantation. 55:752-6; discussion 756-7.

15. Amin, H. I., E. Ai, H. R. McDonald, and R. N. Johnson. 2000. Retinal toxic effects associated with intravitreal fomivirsen. Arch Ophthalmol. 118:426-7.

16. Anders, H. J., and F. D. Goebel. 1999. Neurological manifestations of cytomegalovirus infection in the acquired immunodeficiency syndrome. Int J STD AIDS. 10:151-9.

17. Anderson, K. P., M. C. Fox, V. Brown-Driver, M. J. Martin, and R. F. Azad. 1996. Inhibition of human cytomegalovirus immediate-early gene expression by an antisense oligonucleotide complementary to immediate-early RNA. Antimicrob Agents Chemother. 40:2004-11.

18. Andersson-Ellstrom, A., B. Svennerholm, and L. Forssman. 1995. Prevalence of antibodies to herpes simplex virus types 1 and 2, Epstein- Barr virus and cytomegalovirus in teenage girls. Scand J Infect Dis. 27:315-8.

19. Armitage, J. O. 1994. Bone marrow transplantation. N Engl J Med. 330:827-38.

20. Arnold, J. C., B. C. Portmann, J. G. O'Grady, N. V. Naoumov, G. J. Alexander, and R. Williams. 1992. Cytomegalovirus infection persists in the liver graft in the vanishing bile duct syndrome. Hepatology. 16:285-92.

21. Arribas, J. R., G. A. Storch, D. B. Clifford, and A. C. Tselis. 1996. Cytomegalovirus encephalitis. Ann Intern Med. 125:577-87.

22. Autran, B., G. A. U. L. T. S. Carcelain, C. Blanc, D. Mathez, R. Tubiana, C. Katlama, P. Debre, and J. Leibowitch. 1997. Positive effects of combined antiretroviral therapy on CD4+ T cell homeostasis and function in advanced HIV disease. Science. 277:112-6.

23. Azad, R. F., V. B. Driver, K. Tanaka, R. M. Crooke, and K. P. Anderson. 1993. Antiviral activity of a phosphorothioate oligonucleotide complementary to RNA of the human cytomegalovirus major immediate-early region. Antimicrob Agents Chemother. 37:1945-54. 
24. Bacchi, C. E., C. L. Marsh, J. D. Perkins, R. L. Carithers, J. P. McVicar, K. L. Hudkins, C. D. Benjamin, J. M. Harlan, R. Lobb, and C. E. Alpers. 1993. Expression of vascular cell adhesion molecule (VCAM-1) in liver and pancreas allograft rejection. Am J Pathol. 142:579-91.

25. Bach, M. C., S. P. Bagwell, N. P. Knapp, K. M. Davis, and P. S. Hedstrom. 1985. 9-(1,3-Dihydroxy-2-propoxymethyl)guanine for cytomegalovirus infections in patients with the acquired immunodeficiency syndrome. Ann Intern Med. 103:381-2.

26. Baggiolini, M., B. Dewald, and B. Moser. 1997. Human chemokines: an update. Annu Rev Immunol. 15:675-705.

27. Bailey, T. C., N. A. Ettinger, G. A. Storch, E. P. Trulock, D. W. Hanto, W. C. Dunagan, M. D. Jendrisak, C. S. McCullough, J. L. Kenzora, and W. G. Powderly. 1993. Failure of high-dose oral acyclovir with or without immune globulin to prevent primary cytomegalovirus disease in recipients of solid organ transplants. Am J Med. 95:273-8.

28. Baldanti, F., M. G. Revello, E. Percivalle, and G. Gerna. 1998. Use of the human cytomegalovirus (HCMV) antigenemia assay for diagnosis and monitoring of HCMV infections and detection of antiviral drug resistance in the immunocompromised. $\mathrm{J}$ Clin Virol. 11:51-60.

29. Baldanti, F., M. R. Underwood, S. C. Stanat, K. K. Biron, S. Chou, A. Sarasini, E. Silini, and G. Gerna. 1996. Single amino acid changes in the DNA polymerase confer foscarnet resistance and slow-growth phenotype, while mutations in the UL97-encoded phosphotransferase confer ganciclovir resistance in three double-resistant human cytomegalovirus strains recovered from patients with AIDS. J Virol. 70:1390-5.

30. Baldanti, F., M. R. Underwood, C. L. Talarico, L. Simoncini, A. Sarasini, K. K. Biron, and G. Gerna. 1998. The Cys607-->Tyr change in the UL97 phosphotransferase confers ganciclovir resistance to two human cytomegalovirus strains recovered from two immunocompromised patients. Antimicrob Agents Chemother. 42:444-6.

31. Balthesen, M., M. Messerle, and M. J. Reddehase. 1993. Lungs are a major organ site of cytomegalovirus latency and recurrence. J Virol. 67:5360-6.

32. Barbi, M., S. Binda, V. Primache, and D. Clerici. 1998. Congenital cytomegalovirus infection in a northern Italian region. NEOCMV Group. Eur J Epidemiol. 14:791-6.

33. Barre Sinoussi, F., J. C. Chermann, F. Rey, M. T. Nugeyre, S. Chamaret, J. Gruest, C. Dauguet, C. Axler Blin, F. Vezinet Brun, C. Rouzioux, W. Rozenbaum, and L. Montagnier. 1983. Isolation of a T-lymphotropic retrovirus from a patient at risk for acquired immune deficiency syndrome (AIDS). Science. 220:868-71.

34. Barry, P. A., E. Pratt Lowe, D. J. Alcendor, R. E. Unger, and P. A. Luciw. 1990. Molecular interactions between human immunodeficiency virus type 1 and human cytomegalovirus. Ann N Y Acad Sci. 616:54-63. 
35. Barry, P. A., E. Pratt Lowe, B. M. Peterlin, and P. A. Luciw. 1990. Cytomegalovirus activates transcription directed by the long terminal repeat of human immunodeficiency virus type 1. J Virol. 64:2932-40.

36. Barry, P. A., E. Pratt Lowe, R. E. Unger, M. Marthas, D. J. Alcendor, and P. A. Luciw. 1990. Molecular interactions of cytomegalovirus and the human and simian immunodeficiency viruses. J Med Primatol. 19:327-37.

37. Barry, S. M., M. A. Johnson, and G. Janossy. 2000. Cytopathology or immunopathology? The puzzle of cytomegalovirus pneumonitis revisited. Bone Marrow Transplant. 26:591-7.

38. Basadonna, G. P., A. J. Matas, K. J. Gillingham, W. D. Payne, D. L. Dunn, D. E. Sutherland, P. F. Gores, R. W. Gruessner, and J. S. Najarian. 1993. Early versus late acute renal allograft rejection: impact on chronic rejection. Transplantation. 55:993-5.

39. Beck, S., and B. G. Barrell. 1988. Human cytomegalovirus encodes a glycoprotein homologous to MHC class-I antigens. Nature. 331:269-72.

40. Beersma, M. F., M. J. Bijlmakers, and H. L. Ploegh. 1993. Human cytomegalovirus down-regulates HLA class I expression by reducing the stability of class I H chains. J Immunol. 151:4455-64.

41. Beisser, P. S., G. Grauls, C. A. Bruggeman, and C. Vink. 1999. Deletion of the R78 G protein-coupled receptor gene from rat cytomegalovirus results in an attenuated, syncytium-inducing mutant strain. J Virol. 73:7218-30.

42. Beisser, P. S., S. J. Kaptein, E. Beuken, C. A. Bruggeman, and C. Vink. 1998. The Maastricht strain and England strain of rat cytomegalovirus represent different betaherpesvirus species rather than strains. Virology. 246:341-51.

43. Beisser, P. S., J. S. Kloover, G. E. Grauls, M. J. Blok, C. A. Bruggeman, and C. Vink. 2000. The r144 major histocompatibility complex class I-like gene of rat cytomegalovirus is dispensable for both acute and long-term infection in the immunocompromised host. J Virol. 74:1045-1050.

44. Beisser, P. S., C. Vink, J. G. Van Dam, G. E. L. M. Grauls, S. J. V. Vanherle, and C. A. Bruggeman. 1998. The R33 G protein-coupled receptor gene of rat cytomegalovirus plays an essential role in the pathogenesis of viral infection. Journal of virology. 72:23522363.

45. Berencsi, K., V. Endresz, D. Klurfeld, L. Kari, D. Kritchevsky, and E. Gonczol. 1998. Early atherosclerotic plaques in the aorta following cytomegalovirus infection of mice. Cell Adhes Commun. 5:39-47.

46. Beschorner, W. E., G. M. Hutchins, W. H. Burns, R. Saral, P. J. Tutschka, and G. W. Santos. 1980. Cytomegalovirus pneumonia in bone marrow transplant recipients: miliary and diffuse patterns. Am Rev Respir Dis. 122:107-14. 
47. Beuken, E., R. Slobbe, C. A. Bruggeman, and C. Vink. 1996. Cloning and sequence analysis of the genes encoding DNA polymerase, glycoprotein B, ICP18.5 and major DNA-binding protein of rat cytomegalovirus. J Gen Virol. 77:1559-62.

48. Bevan, I. S., R. A. Daw, P. J. Day, F. A. Ala, and M. R. Walker. 1991. Polymerase chain reaction for detection of human cytomegalovirus infection in a blood donor population. Br J Haematol. 78:94-9.

49. Bia, F. J., B. P. Griffith, C. K. Fong, and G. D. Hsiung. 1983. Cytomegaloviral infections in the guinea pig: experimental models for human disease. Rev Infect Dis. 5:177-95.

50. Billingham, M. E. 1987. Cardiac transplant atherosclerosis. Transplant Proc. 19:19-25.

51. Billingham, M. E. 1992. Histopathology of graft coronary disease. J Heart Lung Transplant. 11:S38-44.

52. Biron, C. A., K. S. Byron, and J. L. Sullivan. 1989. Severe herpesvirus infections in an adolescent without natural killer cells. N Engl J Med. 320:1731-5.

53. Blake, D. R., N. D. Hall, P. A. Bacon, P. A. Dieppe, B. Halliwell, and J. M. Gutteridge. 1983. Effect of a specific iron chelating agent on animal models of inflammation. Ann Rheum Dis. 42:89-93.

54. Blok, M. J., M. H. Christiaans, V. J. Goossens, J. P. van Hooff, P. Sillekens, J. M. Middeldorp, and C. A. Bruggeman. 1999. Early detection of human cytomegalovirus infection after kidney transplantation by nucleic acid sequence-based amplification. Transplantation. 67:1274-7.

55. Blok, M. J., V. J. Goossens, S. J. Vanherle, B. Top, N. Tacken, J. M. Middeldorp, M. H. Christiaans, J. P. van Hooff, and C. A. Bruggeman. 1998. Diagnostic value of monitoring human cytomegalovirus late pp67 mRNA expression in renal-allograft recipients by nucleic acid sequence-based amplification. J Clin Microbiol. 36:1341-6.

56. Bodaghi, B., M. E. Slobbe-van Drunen, A. Topilko, E. Perret, R. C. Vossen, M. C. van Dam-Mieras, D. Zipeto, J. L. Virelizier, P. LeHoang, C. A. Bruggeman, and S. Michelson. 1999. Entry of human cytomegalovirus into retinal pigment epithelial and endothelial cells by endocytosis. Invest Ophthalmol Vis Sci. 40:2598-607.

57. Bodaghi, B., M. E. P. Slobbe-van Drunen, A. Topilko, E. Perret, R. C. R. M. Vossen, M. C. E. van Dam-Mieras, D. Zipeto, J.-L. Virelizier, P. LeHoang, C. A. Bruggeman, and S. Michelson. 1999. Entry of human cytomegalovirus into retinal pigment epithelial and endothelial cells by endocytosis. Invest Ophthalmol Vis Sci. 40:2598-2607.

58. Bodner, L., D. Dayan, Y. Pinto, and I. Hammel. 1993. Characteristics of palatal wound healing in desalivated rats. Arch Oral Biol. 38:17-21.

59. Bodner, L., D. Dayan, D. Rothchild, and I. Hammel. 1991. Extraction wound healing in desalivated rats. J Oral Pathol Med. 20:176-178. 
60. Bodner, L., I. Kaffe, Z. Cohen, and D. Dayan. 1993. Long-term effect of desalivation on extraction wound healing: a densitometric study in rats. Dentomaxillofac Radiol. 22:195-198.

61. Boeckh, M., R. A. Bowden, J. M. Goodrich, M. Pettinger, and J. D. Meyers. 1992. Cytomegalovirus antigen detection in peripheral blood leukocytes after allogeneic marrow transplantation. Blood. 80:1358-64.

62. Boeckh, M., R. A. Bowden, T. Gooley, D. Myerson, and L. Corey. 1999. Successful modification of a pp65 antigenemia-based early treatment strategy for prevention of cytomegalovirus disease in allogeneic marrow transplant recipients. Blood. 93:1781-2.

63. Boeckh, M., T. A. Gooley, D. Myerson, T. Cunningham, G. Schoch, and R. A. Bowden. 1996. Cytomegalovirus pp65 antigenemia-guided early treatment with ganciclovir versus ganciclovir at engraftment after allogeneic marrow transplantation: a randomized double-blind study. Blood. 88:4063-71.

64. Bolovan-Fritts, C. A., E. S. Mocarski, and J. A. Wiedeman. 1999. Peripheral blood CD14(+) cells from healthy subjects carry a circular conformation of latent cytomegalovirus genome. Blood. 93:394-8.

65. Boppana, S. B., R. F. Pass, W. J. Britt, S. Stagno, and C. A. Alford. 1992. Symptomatic congenital cytomegalovirus infection: neonatal morbidity and mortality. Pediatr Infect Dis J. 11:93-9.

66. Bowden, R. A., S. J. Slichter, M. Sayers, D. Weisdorf, M. Cays, G. Schoch, M. Banaji, R. Haake, K. Welk, L. Fisher, and et al. 1995. A comparison of filtered leukocytereduced and cytomegalovirus (CMV) seronegative blood products for the prevention of transfusion- associated CMV infection after marrow transplant. Blood. 86:3598-603.

67. Brockmeyer, C., M. Ulbrecht, D. J. Schendel, E. H. Weiss, G. Hillebrand, K. Burkhardt, W. Land, M. J. Gokel, G. Riethmuller, and H. E. Feucht. 1993. Distribution of cell adhesion molecules (ICAM-1, VCAM-1, ELAM-1) in renal tissue during allograft rejection. Transplantation. 55:610-5.

68. Broers, A. E., R. van Der Holt, J. W. van Esser, J. W. Gratama, S. HenzenLogmans, V. Kuenen-Boumeester, B. Lowenberg, and J. J. Cornelissen. 2000. Increased transplant-related morbidity and mortality in CMV-seropositive patients despite highly effective prevention of CMV disease after allogeneic T-cell-depleted stem cell transplantation. Blood. 95:2240-5.

69. Brosvic, G. M., and N. E. Hoey. 1990. Taste detection and discrimination performance of rats following selective desalivation. Physiol Behav. 48:617-623.

70. Broughton, W. L., H. P. Cupples, and L. M. Parver. 1978. Bilateral retinal detachment following cytomegalovirus retinitis. Arch Ophthalmol. 96:618-9. 
71. Brown, W. R., A. N. Barclay, C. A. Sunderland, and A. F. Williams. 1981. Identification of a glycophorin-like molecisle at the cell surface of rat thymocytes. Nature. 289:456-60.

72. Browne, H., G. Smith, S. Beck, and T. Minson. 1990. A complex between the MHC class I homolog encoded by human cytomegalovirus and beta 2 microglobulin. Nature. 347:770-2.

73. Bruggeman, C. A. 1993. Cytomegalovirus and latency: an overview. Virchows Arch B Cell Pathol Incl Mol Pathol. 64:325-33.

74. Bruggeman, C. A. 1991. Reactivation of latent CMV in the rat. Transplant Proc. 23:22-24.

75. Bruggeman, C. A., W. M. Debie, G. Grauls, G. Majoor, and C. P. van Boven. 1983. Infection of laboratory rats with a new cytomegalo-like virus. Arch Virol. 76:189-199.

76. Bruggeman, C. A., F. Li, and F. S. Stals. 1995. Pathogenicity: animal models. Scand J Infect Dis Suppl. 99:43-50.

77. Bruggeman, C. A., H. Meijer, F. Bosman, and C. P. van Boven. 1985. Biology of rat cytomegalovirus infection. Intervirology. 24:1-9.

78. Bruggeman, C. A., H. Meijer, P. H. Dormans, W. M. Debie, G. E. Grauls, and C. P. van Boven. 1982. Isolation of a cytomegalovirus-like agent from wild rats. Arch Virol. 73:231-241.

79. Bruning, J. H., W. H. Debie, P. H. Dormans, H. Meijer, and C. A. Bruggeman. 1987. The development and characterization of monoclonal antibodies against rat cytomegalovirus induced antigens. Arch Virol. 94:55-70.

80. Bukowski, J. F., J. F. Warner, G. Dennert, and R. M. Welsh. 1985. Adoptive transfer studies demonstrating the antiviral effect of natural killer cells in vivo. J Exp Med. 161:40-52.

81. Cagle, P. T., G. Buffone, V. A. Holland, T. Samo, G. J. Demmler, G. P. Noon, and E. C. Lawrence. 1992. Semiquantitative measurement of cytomegalovirus DNA in lung and heart-lung transplant patients by in vitro DNA amplification. Chest. 101:93-6.

82. Cakaloglu, Y., J. Devlin, J. O'Grady, S. Sutherland, B. C. Portmann, N. Heaton, K. C. Tan, and R. Williams. 1995. Importance of concomitant viral infection during late acute liver allograft rejection. Transplantation. 59:40-5.

83. Campbell, A. E., and J. S. Slater. 1994. Down-regulation of major histocompatibility complex class I synthesis by murine cytomegalovirus early gene expression. J Virol. 68:1805-11.

84. Carlos, T., D. Gordon, D. Fishbein, V. E. Himes, A. Coday, R. Ross, and M. D. Allen. 1992. Vascular cell adhesion molecule-1 is induced on endothelium during acute rejection in human cardiac allografts. J Heart Lung Transplant. 11:1103-8; discussion 1109. 
85. Carlquist, J. F., J. Shelby, Y. L. Shao, J. H. Greenwood, M. E. Hammond, and J. L. Anderson. 1993. Accelerated rejection of murine cardiac allografts by murine cytomegalovirus-infected recipients. Lack of haplotype specificity. J Clin Invest. 91:2602-8. 86. Carlstrom, G., and B. Jalling. 1970. Cytomegalovirus infections in different groups of paediatric patients. Acta Paediatr Scand. 59:303-9.

87. Carretero, M., C. Cantoni, T. Bellon, C. Bottino, R. Biassoni, A. Rodriguez, J. J. Perez-Villar, L. Moretta, A. Moretta, and M. Lopez-Botet. 1997. The CD94 and NKG2-A Ctype lectins covalently assemble to form a natural killer cell inhibitory receptor for HLA class I molecules. Eur J Immunol. 27:563-7.

88. Casareale, D., M. Fiala, C. M. Chang, L. A. Cone, and E. S. Mocarski. 1989. Cytomegalovirus enhances lysis of HIV-infected T lymphoblasts. Int J Cancer. 44:124-30.

89. Casteels, A., A. Naessens, F. Gordts, L. De Catte, A. Bougatef, and W. Foulon. 1999. Neonatal screening for congenital cytomegalovirus infections. J Perinat Med. 27:116-21.

90. Cavanaugh, V. J., R. M. Stenberg, T. L. Staley, H. W. t. Virgin, M. R. MacDonald, S. Paetzold, H. E. Farrell, W. D. Rawlinson, and A. E. Campbell. 1996. Murine cytomegalovirus with a deletion of genes spanning HindIII-J and -I displays altered cell and tissue tropism. J Virol. 70:1365-1374.

91. Chambers, W. H., N. L. Vujanovic, A. B. DeLeo, M. W. Olszowy, R. B. Herberman, and J. C. Hiserodt. 1989. Monoclonal antibody to a triggering structure expressed on rat natural killer cells and adherent lymphokine-activated killer cells. J Exp Med. 169:1373-89.

92. Chapman, T. L., and P. J. Bjorkman. 1998. Characterization of a murine cytomegalovirus class I major histocompatibility complex (MHC) homolog: comparison to MHC molecules and to the human cytomegalovirus MHC homolog. J Virol. 72:460-6.

93. Chee, M. S., A. T. Bankier, S. Beck, R. Bohni, C. M. Brown, R. Cerny, T. Horsnell, C. A. Hutchison, T. Kouzarides, J. A. Martignetti, and et al. 1990. Analysis of the proteincoding content of the sequence of human cytomegalovirus strain AD169. Curr Top Microbiol Immunol. 154:125-69.

94. Chee, M. S., S. C. Satchwell, E. Preddie, K. M. Weston, and B. G. Barrell. 1990. Human cytomegalovirus encodes three $G$ protein-coupled receptor homologs. Nature. 344:774-7.

95. Cherrington, J. M., M. D. Fuller, P. D. Lamy, R. Miner, J. P. Lalezari, S. Nuessle, and W. L. Drew. 1998. In vitro antiviral susceptibilities of isolates from cytomegalovirus retinitis patients receiving first- or second-line cidofovir therapy: relationship to clinical outcome. J Infect Dis. 178:1821-5. 
96. Chiu, B. 1999. Multiple infections in carotid atherosclerotic plaques. Am Heart J. 138:S534-6.

97. Chou, S., G. Marousek, S. Guentzel, S. E. Follansbee, M. E. Poscher, J. P. Lalezari, R. C. Miner, and W. L. Drew. 1997. Evolution of mutations conferring multidrug resistance during prophylaxis and therapy for cytomegalovirus disease. J Infect Dis. 176:786-9.

98. Chou, S., and C. L. Meichsner. 2000. A nine-codon deletion mutation in the cytomegalovirus UL97 phosphotransferase gene confers resistance to ganciclovir. Antimicrob Agents Chemother. 44:183-5.

99. Chou, S., R. C. Miner, and W. L. Drew. 2000. A deletion mutation in region V of the cytomegalovirus DNA polymerase sequence confers multidrug resistance. J Infect Dis. 182:1765-8.

100. Chrisp, P., and S. P. Clissold. 1991. Foscarnet. A review of its antiviral activity, pharmacokinetic properties and therapeutic use in immunocompromised patients with cytomegalovirus retinitis. Drugs. 41:104-29.

101. Cihlar, T., and M. S. Chen. 1996. Identification of enzymes catalyzing two-step phosphorylation of cidofovir and the effect of cytomegalovirus infection on their activities in host cells. Mol Pharmacol. 50:1502-10.

102. Cihlar, T., M. D. Fuller, A. S. Mulato, and J. M. Cherrington. 1998. A point mutation in the human cytomegalovirus DNA polymerase gene selected in vitro by cidofovir confers a slow replication phenotype in cell culture. Virology. 248:382-93.

103. Cinatl, J., Jr., J. Cinatl, H. Rabenau, H. O. Gumbel, B. Kornhuber, and H. W. Doerr. 1994. In vitro inhibition of human cytomegalovirus replication by desferrioxamine. Antiviral Res. 25:73-7.

104. Cinatl, J., J. Cinatl, B. Weber, H. Rabenau, H. O. Gumbel, J. F. Chenot, M. Scholz, A. Encke, and H. W. Doerr. 1995. In vitro inhibition of human cytomegalovirus replication in human foreskin fibroblasts and endothelial cells by ascorbic acid 2-phosphate. Antiviral Res. 27:405-18.

105. Cinatl, J., Jr., F. Hoffmann, J. Cinatl, B. Weber, M. Scholz, H. Rabenau, F. Stieneker, H. Kabickova, M. Blasko, and H. W. Doerr. 1995. In vitro inhibition of human cytomegalovirus replication by calcium trinatrium diethylenetriaminepentaacetic acid. Antiviral Res. 31:23-34.

106. Coats, D. K., G. J. Demmler, E. A. Paysse, L. T. Du, and C. Libby. 2000. Ophthalmologic findings in children with congenital cytomegalovirus infection. J Aapos. 4:110-6. 
107. Cochereau Massin, I., P. Lehoang, M. Lautier Frau, L. Zazoun, P. Marcel, M. Robinet, S. Matheron, C. Katlama, S. Gharakhanian, W. Rozenbaum, and et al. 1991. Efficacy and tolerance of intravitreal ganciclovir in cytomegalovirus retinitis in acquired immune deficiency syndrome. Ophthalmology. 98:1348-53.

108. Cohen, B. A., J. C. McArthur, S. Grohman, B. Patterson, and J. D. Glass. 1993. Neurologic prognosis of cytomegalovirus polyradiculomyelopathy in AIDS. Neurology. 43:493-9.

109. Collier, A. C., J. D. Meyers, L. Corey, V. L. Murphy, P. L. Roberts, and H. H. Handsfield. 1987. Cytomegalovirus infection in homosexual men. Relationship to sexual practices, antibody to human immunodeficiency virus, and cell-mediated immunity. Am J Med. 82:593-601.

110. Collins, P. 1983. The spectrum of antiviral activities of acyclovir in vitro and in vivo. J Antimicrob Chemother. 12:19-27.

111. Collins, T., C. Pomeroy, and M. C. Jordan. 1993. Detection of latent cytomegalovirus DNA in diverse organs of mice. J Infect Dis. 168:725-9.

112. Compton, T., R. R. Nepomuceno, and D. M. Nowlin. 1992. Human cytomegalovirus penetrates host cells by $\mathrm{pH}$-independent fusion at the cell surface. Virology. 191:387-95.

113. Compton, T., D. M. Nowlin, and N. R. Cooper. 1993. Initiation of human cytomegalovirus infection requires initial interaction with cell surface heparan sulfate. Virology. 193:834-41.

114. Cosman, D., N. Fanger, L. Borges, M. Kubin, W. Chin, L. Peterson, and M. L. Hsu. 1997. A novel immunoglobulin superfamily receptor for cellular and viral MHC class I molecules. Immunity. 7:273-82.

115. Cremer, K. J., S. B. Spring, and J. Gruber. 1990. Role of human immunodeficiency virus type 1 and other viruses in malignancies associated with acquired immunodeficiency disease syndrome. J Natl Cancer Inst. 82:1016-24.

116. Cretney, E., M. A. Degli-Esposti, E. H. Densley, H. E. Farrell, N. J. Davis-Poynter, and M. J. Smyth. 1999. m144, a murine cytomegalovirus (MCMV)-encoded major histocompatibility complex class I homolog, confers tumor resistance to natural killer cellmediated rejection. J Exp Med. 190:435-44.

117. Crowe, S. M., J. B. Carlin, K. I. Stewart, C. R. Lucas, and J. F. Hoy. 1991. Predictive value of CD4 lymphocyte numbers for the development of opportunistic infections and malignancies in HIV-infected persons. J Acquir Immune Defic Syndr. 4:7706.

118. Cundy, K. C. 1999. Clinical pharmacokinetics of the antiviral nucleotide analogues cidofovir and adefovir. Clin Pharmacokinet. 36:127-43. 
119. Cundy, K. C., B. G. Petty, J. Flaherty, P. E. Fisher, M. A. Polis, M. Wachsman, P. S. Lietman, J. P. Lalezari, M. J. Hitchcock, and H. S. Jaffe. 1995. Clinical pharmacokinetics of cidofovir in human immunodeficiency virus-infected patients. Antimicrob Agents Chemother. 39:1247-52.

120. Dangler, C. A., S. E. Baker, M. Kariuki Njenga, and S. H. Chia. 1995. Murine cytomegalovirus-associated arteritis. Vet Pathol. 32:127-33.

121. Davies, S. M., X. O. Shu, B. R. Blazar, A. H. Filipovich, J. H. Kersey, W. Krivit, J. McCullough, W. J. Miller, N. K. Ramsay, M. Segall, and et al. 1995. Unrelated donor bone marrow transplantation: influence of HLA A and B incompatibility on outcome. Blood. 86:1636-42.

122. Davignon, J. L., P. Castanie, J. A. Yorke, N. Gautier, D. Clement, and C. Davrinche. 1996. Anti-human cytomegalovirus activity of cytokines produced by CD4+ Tcell clones specifically activated by IE1 peptides in vitro. J Virol. 70:2162-9.

123. Davis, M. G., S. C. Kenney, J. Kamine, J. S. Pagano, and E. S. Huang. 1987. Immediate-early gene region of human cytomegalovirus trans-activates the promoter of human immunodeficiency virus. Proc Natl Acad Sci U S A. 84:8642-6.

124. Davis-Poynter, N. J., D. M. Lynch, H. Vally, G. R. Shellam, W. D. Rawlinson, B. G. Barrell, and H. E. Farrell. 1997. Identification and characterization of a $G$ protein-coupled receptor homolog encoded by murine cytomegalovirus. J Virol. 71:1521-9.

125. De Clercq, E., A. Holy, I. Rosenberg, T. Sakuma, J. Balzarini, and P. C. Maudgal. 1986. A novel selective broad-spectrum anti-DNA virus agent. Nature. 323:464-467.

126. De Clercq, E., T. Sakuma, M. Baba, R. Pauwels, J. Balzarini, I. Rosenberg, and A. Holy. 1987. Antiviral activity of phosphonylmethoxyalkyl derivatives of purine and pyrimidines. Antiviral Res. 8:261-72.

127. de Smet, M. D., C. J. Meenken, and G. J. van den Horn. 1999. Fomivirsen - a phosphorothioate oligonucleotide for the treatment of $\mathrm{CMV}$ retinitis. Ocul Immunol Inflamm. 7:189-98.

128. Demmler, G. J. 1991. Infectious Diseases Society of America and Centers for Disease Control. Summary of a workshop on surveillance for congenital cytomegalovirus disease. Rev Infect Dis. 13:315-29.

129. Deorari, A. K., S. Broor, R. S. Maitreyi, D. Agarwal, H. Kumar, V. K. Paul, and M. Singh. 2000. Incidence, clinical spectrum, and outcome of intrauterine infections in neonates. J Trop Pediatr. 46:155-9.

130. Detrick, B., C. N. Nagineni, L. R. Grillone, K. P. Anderson, S. P. Henry, and J. J. Hooks. 2001. Inhibition of human cytomegalovirus replication in a human retinal epithelial cell model by antisense oligonucleotides. Invest Ophthalmol Vis Sci. 42:163-9. 
131. Dickinson, B. I., M. L. Gora-Harper, S. A. McCraney, and M. Gosland. 1996. Studies evaluating high-dose acyclovir, intravenous immune globulin, and cytomegalovirus hyperimmunoglobulin for prophylaxis against cytomegalovirus in kidney transplant recipients. Ann Pharmacother. 30:1452-64.

132. Dieterich, D. T., and M. Rahmin. 1991. Cytomegalovirus colitis in AIDS: presentation in 44 patients and a review of the literature. J Acquir Immune Defic Syndr. 4 Suppl 1:S29-35.

133. Dijkstra, C. D., E. A. Dopp, P. Joling, and G. Kraal. 1985. The heterogeneity of mononuclear phagocytes in lymphoid organs: distinct macrophage subpopulations in the rat recognized by monoclonal antibodies ED1, ED2 and ED3. Immunology. 54:589-99.

134. Drew, W. L., D. Ives, J. P. Lalezari, C. Crumpacker, S. E. Follansbee, S. A. Spector, C. A. Benson, D. N. Friedberg, L. Hubbard, M. J. Stempien, and et al. 1995. Oral ganciclovir as maintenance treatment for cytomegalovirus retinitis in patients with AIDS. Syntex Cooperative Oral Ganciclovir Study Group. N Engl J Med. 333:615-20.

135. Drew, W. L., R. C. Miner, D. F. Busch, S. E. Follansbee, J. Gullett, S. G. Mehalko, S. M. Gordon, W. F. Owen, Jr., T. R. Matthews, W. C. Buhles, and et al. 1991. Prevalence of resistance in patients receiving ganciclovir for serious cytomegalovirus infection. J Infect Dis. 163:716-9.

136. Drew, W. L., L. Mintz, R. C. Miner, M. Sands, and B. Ketterer. 1981. Prevalence of cytomegalovirus infection in homosexual men. J Infect Dis. 143:188-92.

137. Dummer, J. S., A. Hardy, A. Poorsattar, and M. Ho. 1983. Early infections in kidney, heart, and liver transplant recipients on cyclosporine. Transplantation. 36:259-67.

138. Dummer, J. S., L. T. White, M. Ho, B. P. Griffith, R. L. Hardesty, and H. T. Bahnson. 1985. Morbidity of cytomegalovirus infection in recipients of heart or heart-lung transplants who received cyclosporine. J Infect Dis. 152:1182-91.

139. Dummer, S., A. Lee, M. K. Breinig, R. Kormos, M. Ho, and B. Griffith. 1994. Investigation of cytomegalovirus infection as a risk factor for coronary atherosclerosis in the explanted hearts of patients undergoing heart transplantation. J Med Virol. 44:305-9.

140. Eckle, T., L. Prix, G. Jahn, T. Klingebiel, R. Handgretinger, B. Selle, and K. Hamprecht. 2000. Drug-resistant human cytomegalovirus infection in children after allogeneic stem cell transplantation may have different clinical outcomes. Blood. 96:32869.

141. Eddleston, M., S. Peacock, M. Juniper, and D. A. Warrell. 1997. Severe cytomegalovirus infection in immunocompetent patients. Clin Infect Dis. 24:52-6.

142. Einsele, H., G. Ehninger, H. Hebart, K. M. Wittkowski, U. Schuler, G. Jahn, P. Mackes, M. Herter, T. Klingebiel, J. Loffler, and et al. 1995. Polymerase chain reaction monitoring reduces the incidence of cytomegalovirus disease and the duration and side effects of antiviral therapy after bone marrow transplantation. Blood. 86:2815-20. 
143. Elkins, C. C., W. H. Frist, J. S. Dummer, J. R. Stewart, W. H. Merrill, K. A. Carden, and H. W. Bender. 1993. Cytomegalovirus disease after heart transplantation: is acyclovir prophylaxis indicated? Ann Thorac Surg. 56:1267-72; discussion 1272-3.

144. Emanuel, D. 1990. Treatment of cytomegalovirus disease. Semin Hematol. 27:227.

145. Enright, H., R. Haake, D. Weisdorf, N. Ramsay, P. McGlave, J. Kersey, W. Thomas, D. McKenzie, and W. Miller. 1993. Cytomegalovirus pneumonia after bone marrow transplantation. Risk factors and response to therapy. Transplantation. 55:133946.

146. Erice, A., S. Chou, K. K. Biron, S. C. Stanat, H. H. Balfour, Jr., and M. C. Jordan. 1989. Progressive disease due to ganciclovir-resistant cytomegalovirus in immunocompromised patients. N Engl J Med. 320:289-93.

147. Evans, P. C., A. Soin, T. G. Wreghitt, C. J. Taylor, D. G. Wight, and G. J. Alexander. 2000. An association between cytomegalovirus infection and chronic rejection after liver transplantation. Transplantation. 69:30-5.

148. Everett, J. P., R. E. Hershberger, D. J. Norman, S. Chou, R. M. Ratkovec, A. Cobanoglu, G. Y. Ott, and J. D. Hosenpud. 1992. Prolonged cytomegalovirus infection with viremia is associated with development of cardiac allograft vasculopathy. J Heart Lung Transplant. 11:S133-7.

149. Faggiotto, A., and R. Ross. 1984. Studies of hypercholesterolemia in the nonhuman primate. II. Fatty streak conversion to fibrous plaque. Arteriosclerosis. 4:34156.

150. Faggiotto, A., R. Ross, and L. Harker. 1984. Studies of hypercholesterolemia in the nonhuman primate. I. Changes that lead to fatty streak formation. Arteriosclerosis. 4:323-40.

151. Fahnestock, M. L., J. L. Johnson, R. M. Feldman, J. M. Neveu, W. S. Lane, and P. J. Bjorkman. 1995. The MHC class I homolog encoded by human cytomegalovirus binds endogenous peptides. Immunity. 3:583-90.

152. Faizi Khan, R., S. Mori, Y. Eizuru, K. Kumura Ishii, and Y. Minamishima. 1998. Genetic analysis of a ganciclovir-resistant human cytomegalovirus mutant. Antiviral Res. 40:95-103.

153. Fanning, M. M., S. E. Read, M. Benson, S. Vas, A. Rachlis, V. Kozousek, C. Mortimer, P. Harvey, C. Schwartz, E. Chew, and et al. 1990. Foscarnet therapy of cytomegalovirus retinitis in AIDS. J Acquir Immune Defic Syndr. 3:472-9.

154. Farrell, H. E., and G. R. Shellam. 1991. Protection against murine cytomegalovirus infection by passive transfer of neutralizing and non-neutralizing monoclonal antibodies. $J$ Gen Virol. 72:149-156. 
155. Farrell, H. E., H. Vally, D. M. Lynch, P. Fleming, G. R. Shellam, A. A. Scalzo, and N. J. Davis Poynter. 1997. Inhibition of natural killer cells by a cytomegalovirus MHC class I homolog in vivo. Nature. 386:510-4.

156. Fauci, A. S. 1988. The human immunodeficiency virus: infectivity and mechanisms of pathogenesis. Science. 239:617-22.

157. Faulds, D., and R. C. Heel. 1990. Ganciclovir. A review of its antiviral activity, pharmacokinetic properties and therapeutic efficacy in cytomegalovirus infections. Drugs. 39:597-638.

158. Felsenstein, D., D. A. DJ, M. S. Hirsch, D. A. Neumeyer, D. M. Cederberg, P. de Miranda, and R. T. Schooley. 1985. Treatment of cytomegalovirus retinitis with 9-[2hydroxy-1-(hydroxymethyl)ethoxymethyl]guanine. Ann Intern Med. 103:377-80.

159. Fiala, M., L. A. Cone, R. Christopher, V. Kermani, and J. A. Gornbein. 1991. Human immunodeficiency virus type 1 antigenaemia is enhanced in patients with disseminated cytomegalovirus infection and deficient T lymphocytes. Res Immunol. 142:815-9.

160. Fiala, M., J. D. Mosca, P. Barry, P. A. Luciw, and H. V. Vinters. 1991. Multi-step pathogenesis of AIDS--role of cytomegalovirus. Res Immunol. 142:87-95.

161. Fiala, M., E. J. Singer, M. C. Graves, W. W. Tourtellotte, J. A. Stewart, C. A. Schable, R. H. Rhodes, and H. V. Vinters. 1993. AIDS dementia complex complicated by cytomegalovirus encephalopathy. J Neurol. 240:223-31.

162. Fish, K. N., W. Britt, and J. A. Nelson. 1996. A novel mechanism for persistence of human cytomegalovirus in macrophages. J Virol. 70:1855-62.

163. Fish, K. N., C. Soderberg-Naucler, L. K. Mills, S. Stenglein, and J. A. Nelson. 1998. Human cytomegalovirus persistently infects aortic endothelial cells. J Virol. 72:56618.

164. Fong, C. K., F. Bia, and G. D. Hsiung. 1980. Ultrastructural development and persistence of guinea pig cytomegalovirus in duet cells of guinea pig submaxillary gland. Arch Virol. 64:97-108.

165. Fong, C. K., H. Lucia, F. J. Bia, and G. D. Hsiung. 1983. Histopathologic and ultrastructural studies of disseminated cytomegalovirus infection in strain 2 guinea pigs. Lab Invest. 49:183-94.

166. Fowler, K. B., A. J. Dahle, S. B. Boppana, and R. F. Pass. 1999. Newborn hearing screening: will children with hearing loss caused by congenital cytomegalovirus infection be missed? J Pediatr. 135:60-4.

167. Fowler, K. B., F. P. McCollister, A. J. Dahle, S. Boppana, W. J. Britt, and R. F. Pass. 1997. Progressive and fluctuating sensorineural hearing loss in children with asymptomatic congenital cytomegalovirus infection. J Pediatr. 130:624-30. 
168. Fowler, K. B., and R. F. Pass. 1991. Sexually transmitted diseases in mothers of neonates with congenital cytomegalovirus infection. J Infect Dis. 164:259-64.

169. Fowler, K. B., S. Stagno, R. F. Pass, W. J. Britt, T. J. Boll, and C. A. Alford. 1992. The outcome of congenital cytomegalovirus infection in relation to maternal antibody status. N Engl J Med. 326:663-667.

170. Freeman, W. R., D. E. Henderly, W. L. Wan, D. Causey, M. Trousdale, R. L. Green, and N. A. Rao. 1987. Prevalence, pathophysiology, and treatment of rhegmatogenous retinal detachment in treated cytomegalovirus retinitis. Am J Ophthalmol. 103:527-36.

171. Fries, B. C., S. Chou, M. Boeckh, and B. Torok-Storb. 1994. Frequency distribution of cytomegalovirus envelope glycoprotein genotypes in bone marrow transplant recipients. J Infect Dis. 169:769-74.

172. Fuggle, S. V., and D. D. Koo. 1998. Cell adhesion molecules in clinical renal transplantation. Transplantation. 65:763-9.

173. Fujinami, R. S., J. A. Nelson, L. Walker, and M. B. Oldstone. 1988. Sequence homology and immunologic cross-reactivity of human cytomegalovirus with HLA-DR beta chain: a means for graft rejection and immunosuppression. J Virol. 62:100-5.

174. Gallant, J. E., R. D. Moore, D. D. Richman, J. Keruly, and R. E. Chaisson. 1992. Incidence and natural history of cytomegalovirus disease in patients with advanced human immunodeficiency virus disease treated with zidovudine. The Zidovudine Epidemiology Study Group. J Infect Dis. 166:1223-7.

175. Gallo, R. C., S. Z. Salahuddin, M. Popovic, G. M. Shearer, M. Kaplan, B. F. Haynes, T. J. Palker, R. Redfield, J. Oleske, B. Safai, and et al. 1984. Frequent detection and isolation of cytopathic retroviruses (HTLV-III) from patients with AIDS and at risk for AIDS. Science. 224:500-3.

176. Gane, E., F. Saliba, G. J. Valdecasas, J. O'Grady, M. D. Pescovitz, S. Lyman, and C. A. Robinson. 1997. Randomised trial of efficacy and safety of oral ganciclovir in the prevention of cytomegalovirus disease in liver-transplant recipients. The Oral Ganciclovir International Transplantation Study Group [corrected]. Lancet. 350:1729-33.

177. Garcia, C. R., F. J. Torriani, and W. R. Freeman. 1998. Cidofovir in the treatment of cytomegalovirus (CMV) retinitis. Ocul Immunol Inflamm. 6:195-203.

178. Gavalda, J., J. de Otero, E. Murio, V. Vargas, J. Rossello, I. Calico, C. Margarit, and A. Pahissa. 1997. Two grams daily of oral acyclovir reduces the incidence of cytomegalovirus disease in CMV-seropositive liver transplant recipients. Transpl Int. 10:462-5.

179. Geist, L. J., M. M. Monick, M. F. Stinski, and G. W. Hunninghake. 1991. The immediate early genes of human cytomegalovirus upregulate expression of the interleukin2 and interleukin-2 receptor genes. Am J Respir Cell Mol Biol. 5:292-6. 
180. George, M. J., D. R. Snydman, B. G. Werner, N. N. Dougherty, J. Griffith, R. H. Rohrer, R. Freeman, R. Jenkins, and W. D. Lewis. 1993. Use of ganciclovir plus cytomegalovirus immune globulin to treat CMV pneumonia in orthotopic liver transplant recipients. The Boston Center for Liver Transplantation CMVIG-Study Group. Transplant Proc. 25:22-4.

181. Gerna, G., F. Baldanti, M. Zavattoni, A. Sarasini, E. Percivalle, and M. G. Revello. 1992. Monitoring of ganciclovir sensitivity of multiple human cytomegalovirus strains coinfecting blood of an AIDS patient by an immediate-early antigen plaque assay. Antiviral Res. 19:333-45.

182. Gerna, G., E. Percivalle, F. Baldanti, S. Sozzani, P. Lanzarini, E. Genini, D. Lilleri, and M. G. Revello. 2000. Human cytomegalovirus replicates abortively in polymorphonuclear leukocytes after transfer from infected endothelial cells via transient microfusion events. J Virol. 74:5629-38.

183. Ghebrekidan, H., U. Ruden, S. Cox, B. Wahren, and M. Grandien. 1999. Prevalence of herpes simplex virus types 1 and 2, cytomegalovirus, and varicella-zoster virus infections in Eritrea. J Clin Virol. 12:53-64.

184. Gibbs, P., L. M. Berkley, E. M. Bolton, J. D. Briggs, and J. A. Bradley. 1993. Adhesion molecule expression (ICAM-1, VCAM-1, E-selectin and PECAM) in human kidney allografts. Transpl Immunol. 1:109-13.

185. Gibson, W. 1996. Structure and assembly of the virion. Intervirology. 39:389-400.

186. Gilbert, M. J., S. R. Riddell, B. Plachter, and P. D. Greenberg. 1996. Cytomegalovirus selectively blocks antigen processing and presentation of its immediateearly gene product. Nature. 383:720-2.

187. Goodrich, J. M., M. Boeckh, and R. Bowden. 1994. Strategies for the prevention of cytomegalovirus disease after marrow transplantation. Clin Infect Dis. 19:287-98.

188. Goodrich, J. M., M. Mori, C. A. Gleaves, C. Du Mond, M. Cays, D. F. Ebeling, W. C. Buhles, B. DeArmond, and J. D. Meyers. 1991. Early treatment with ganciclovir to prevent cytomegalovirus disease after allogeneic bone marrow transplantation. $\mathrm{N}$ Engl J Med. 325:1601-7.

189. Goral, S., C. Ynares, S. Dummer, and J. H. Helderman. 1996. Acyclovir prophylaxis for cytomegalovirus disease in high-risk renal transplant recipients: is it effective? Kidney Int Suppl. 57:S62-5.

190. Gordin. 1984. Advers reactions to trimethoprim-sulfamethoxazole in patients with the acquired immunodeficiency syndrome. Ann intern Med. 100:495-499.

191. Grafe, M. R., and C. A. Wiley. 1989. Spinal cord and peripheral nerve pathology in AIDS: the roles of cytomegalovirus and human immunodeficiency virus. Ann Neurol. 25:561-6. 
192. Gratacap-Cavallier, B., J. L. Bosson, P. Morand, N. Dutertre, B. Chanzy, P. S. Jouk, C. Vandekerckhove, P. Cart-Lamy, and J. M. Seigneurin. 1998. Cytomegalovirus seroprevalence in French pregnant women: parity and place of birth as major predictive factors. Eur J Epidemiol. 14:147-52.

193. Grattan, M. T., C. E. Moreno Cabral, V. A. Starnes, P. E. Oyer, E. B. Stinson, and N. E. Shumway. 1989. Cytomegalovirus infection is associated with cardiac allograft rejection and atherosclerosis. Jama. 261:3561-6.

194. Greenberg, M. S., M. Glick, L. Nghiem, J. C. Stewart, R. Hodinka, and G. Dubin. 1997. Relationship of cytomegalovirus to salivary gland dysfunction in HIV-infected patients. Oral Surg Oral Med Oral Pathol Oral Radiol Endod. 83:334-9.

195. Grefte, A., M. van der Giessen, W. van Son, and T. H. The. 1993. Circulating cytomegalovirus (CMV)-infected endothelial cells in patients with an active CMV infection. $\mathrm{J}$ Infect Dis. 167:270-7.

196. Griffith, B. P., H. C. Isom, and J. T. Lavallee. 1990. Cellular localization of cytomegalovirus nucleic acids in guinea pig salivary glands by in situ hybridization. J Virol Methods. 27:145-57.

197. Griffiths, P. D. 1994. Cytomegalovirus, p. 69-108. In A. J. Zuckerman and J. E. Banatvala and J. R. Pattison (ed.), Principles and practice of clinical virology, vol. 3. John Wiley \& Sons Ltd, Chichester, West Sussex.

198. Group, C. D. T. S. 1986. Treatment of serious cytomegalovirus infections with 9(1,3-dihydroxy-2-propoxymethyl)guanine in patients with AIDS and other immunodeficiencies. N Engl J Med. 314:801-5.

199. Grundy, J. E., and K. L. Downes. 1993. Up-regulation of LFA-3 and ICAM-1 on the surface of fibroblasts infected with cytomegalovirus. Immunology. 78:405-12.

200. Grzimek, N. K., D. Dreis, S. Schmalz, and M. J. Reddehase. 2001. Random, Asynchronous, and Asymmetric Transcriptional Activity of Enhancer-Flanking Major Immediate-Early Genes ie1/3 and ie2 during Murine Cytomegalovirus Latency in the Lungs. J Virol. 75:2692-2705.

201. Gumbel, H., J. Cinatl, Jr., H. Rabenau, J. U. Vogel, H. W. Doerr, and C. Ohrloff. 1995. Selective inhibition of replication of human cytomegalovirus by desferrioxamine in vitro and in vivo (case report). Ophthalmologe. 92:840-3.

202. Haagmans, B. L., A. J. van den Eertwegh, E. Claassen, M. C. Horzinek, and V. E. Schijns. 1994. Tumour necrosis factor-alpha production during cytomegalovirus infection in immunosuppressed rats. J Gen Virol. 75:779-87.

203. Hahn, G., R. Jores, and E. S. Mocarski. 1998. Cytomegalovirus remains latent in a common precursor of dendritic and myeloid cells. Proc Natl Acad Sci U S A. 95:3937-42. 
204. Halwachs-Baumann, G., B. Genser, M. Danda, H. Engele, H. Rosegger, B. Folsch, U. Maurer, H. Lackner, and M. Truschnig-Wilders. 2000. Screening and diagnosis of congenital cytomegalovirus infection: a 5-y study. Scand J Infect Dis. 32:137-42.

205. Hancock, W. H., W. D. Whitley, S. G. Tullius, U. W. Heemann, B. Wasowska, W. M. Baldwin, and N. L. Tilney. 1993. Cytokines, adhesion molecules, and the pathogenesis of chronic rejection of rat renal allografts. Transplantation. 56:643-50.

206. Harada, K., Y. Eizuru, Y. Isashiki, S. Ihara, and Y. Minamishima. 1997. Genetic analysis of a clinical isolate of human cytomegalovirus exhibiting resistance against both ganciclovir and cidofovir. Arch Virol. 142:215-25.

207. Hassan Walker, A. F., A. V. Cope, P. D. Griffiths, and V. C. Emery. 1998. Transcription of the human cytomegalovirus natural killer decoy gene, UL18, in vitro and in vivo. J Gen Virol. 79:2113-6.

208. Hawley, D. A., J. F. Schaefer, D. M. Schulz, and J. Muller. 1983. Cytomegalovirus encephalitis in acquired immunodeficiency syndrome. Am J Clin Pathol. 80:874-7.

209. Hayry, P., H. Isoniemi, S. Yilmaz, A. Mennander, K. Lemstrom, A. RaisanenSokolowski, P. Koskinen, J. Ustinov, I. Lautenschlager, E. Taskinen, and et al. 1993. Chronic allograft rejection. Immunol Rev. 134:33-81.

210. Hebart, H., and H. Einsele. 1998. Diagnosis and treatment of cytomegalovirus infection. Curr Opin Hematol. 5:483-487.

211. Hendrix, M. G., M. M. Salimans, C. P. van Boven, and C. A. Bruggeman. 1990. High prevalence of latently present cytomegalovirus in arterial walls of patients suffering from grade III atherosclerosis. Am J Pathol. 136:23-8.

212. Hendrix, R. M., M. Wagenaar, R. L. Slobbe, and C. A. Bruggeman. 1997. Widespread presence of cytomegalovirus DNA in tissues of healthy trauma victims. J Clin Pathol. 50:59-63.

213. Hengel, H., J. O. Koopmann, T. Flohr, W. Muranyi, E. Goulmy, G. J. Hammerling, U. H. Koszinowski, and F. Momburg. 1997. A viral ER-resident glycoprotein inactivates the MHC-encoded peptide transporter. Immunity. 6:623-32.

214. Hennis, H. L., A. A. Scott, and D. J. Apple. 1989. Cytomegalovirus retinitis. Surv Ophthalmol. 34:193-203.

215. Henson, D., and C. Neapolitan. 1970. Pathogenesis of chronic mouse cytomegalovirus infection in submaxillary glands of C3H mice. Am J Pathol. 58:255-67.

216. Henson, D., R. D. Smith, J. Gehrke, and C. Neapolitan. 1967. Effect of cortisone on nonfatal mouse cytomegalovirus infection. Am J Pathol. 51:1001-11.

217. Henson, D., and A. J. Strano. 1972. Mouse cytomegalovirus. Necrosis of infected and morphologically normal submaxillary gland acinar cells during termination of chronic infection. Am J Pathol. 68:183-202. 
218. Hertenstein, B., W. Hampl, D. Bunjes, M. Wiesneth, C. Duncker, U. Koszinowski, H. Heimpel, R. Arnold, and T. Mertens. 1995. In vivo/ex vivo T cell depletion for GVHD prophylaxis influences onset and course of active cytomegalovirus infection and disease after BMT. Bone Marrow Transplant. 15:387-93.

219. High, K. P. 1999. Atherosclerosis and infection due to Chlamydia pneumoniae or cytomegalovirus: weighing the evidence. Clin Infect Dis. 28:746-9.

220. Hill, P. A., I. W. Main, and R. C. Atkins. 1995. ICAM-1 and VCAM-1 in human renal allograft rejection. Kidney Int. 47:1383-91.

221. Hizel, S., S. Parker, and U. Onde. 1999. Seroprevalence of cytomegalovirus infection among children and females in Ankara, Turkey, 1995. Pediatr Int. 41:506-509.

222. Ho, M. 1991. Cytomegalovirus -Biology and infection-, 2nd ed. Plenium Publishing Corporation, New York.

223. Holland, N. R., C. Power, V. P. Mathews, J. D. Glass, M. Forman, and J. C. McArthur. 1994. Cytomegalovirus encephalitis in acquired immunodeficiency syndrome (AIDS). Neurology. 44:507-14.

224. Holmberg, L. A., M. Boeckh, H. Hooper, W. Leisenring, S. Rowley, S. Heimfeld, O. Press, D. G. Maloney, P. McSweeney, L. Corey, R. T. Maziarz, F. R. Appelbaum, and W. Bensinger. 1999. Increased incidence of cytomegalovirus disease after autologous CD34selected peripheral blood stem cell transplantation. Blood. 94:4029-35.

225. Horowitz, H. W., E. E. Telzak, K. A. Sepkowitz, and G. P. Wormser. 1998. Human immunodeficiency virus infection, Part II. Dis Mon. 44:677-716.

226. Hosenpud, J. D. 1999. Coronary artery disease after heart transplantation and its relation to cytomegalovirus. Am Heart J. 138:S469-72.

227. Hudson, J. B. 1979. The murine cytomegalovirus as a model for the study of viral pathogenesis and persistent infections. Arch Virol. 62:1-29.

228. Humar, A., K. J. Gillingham, W. D. Payne, D. L. Dunn, D. E. Sutherland, and A. J. Matas. 1999. Association between cytomegalovirus disease and chronic rejection in kidney transplant recipients. Transplantation. 68:1879-83.

229. Humar, A., S. Wood, J. Lipton, H. Messner, J. Meharchand, A. McGeer, K. MacDonald, and T. Mazzulli. 1998. Effect of cytomegalovirus infection on 1-year mortality rates among recipients of allogeneic bone marrow transplants. Clin Infect Dis. 26:606-10.

230. Hunninghake, G. W., M. M. Monick, and L. J. Geist. 1999. Cytomegalovirus infection. Regulation of inflammation. Am J Respir Cell Mol Biol. 21:150-2.

231. Ibanez, C. E., R. Schrier, P. Ghazal, C. Wiley, and J. A. Nelson. 1991. Human cytomegalovirus productively infects primary differentiated macrophages. J Virol. 65:65818. 
232. Ippoliti, C., A. Morgan, D. Warkentin, K. van Besien, R. Mehra, I. Khouri, S. Giralt, J. Gajewski, R. Champlin, B. Andersson, and D. Przepiorka. 1997. Foscarnet for prevention of cytomegalovirus infection in allogeneic marrow transplant recipients unable to receive ganciclovir. Bone Marrow Transplant. 20:491-5.

233. Isoniemi, H., E. Taskinen, and P. Hayry. 1994. Histological chronic allograft damage index accurately predicts chronic renal allograft rejection. Transplantation. 58:1195-8.

234. Isoniemi, H. M., L. Krogerus, E. von Willebrand, E. Taskinen, J. Ahonen, and P. Hayry. 1992. Histopathological findings in well-functioning, long-term renal allografts. Kidney Int. 41:155-60.

235. Iwamoto, G. K., M. M. Monick, B. D. Clark, P. E. Auron, M. F. Stinski, and G. W. Hunninghake. 1990. Modulation of interleukin 1 beta gene expression by the immediate early genes of human cytomegalovirus. J Clin Invest. 85:1853-7.

236. Jacobson, M. A. 1992. Review of the toxicities of foscarnet. J Acquir Immune Defic Syndr. 5:S11-7.

237. Jacobson, M. A. 1997. Treatment of cytomegalovirus retinitis in patients with the acquired immunodeficiency syndrome. N Engl J Med. 337:105-14.

238. Jacobson, M. A., W. L. Drew, J. Feinberg, O. D. JJ, P. V. Whitmore, R. D. Miner, and D. Parenti. 1991. Foscarnet therapy for ganciclovir-resistant cytomegalovirus retinitis in patients with AIDS. J Infect Dis. 163:1348-51.

239. Jacobson, M. A., and M. French. 1998. Altered natural history of AIDS-related opportunistic infections in the era of potent combination antiretroviral therapy. Aids. 12:S157-63.

240. Jacobson, M. A., and J. Mills. 1988. Serious cytomegalovirus disease in the acquired immunodeficiency syndrome (AIDS). Clinical findings, diagnosis, and treatment. Ann Intern Med. 108:585-94.

241. Jacobson, M. A., M. Zegans, P. R. Pavan, O. D. JJ, F. Sattler, N. Rao, S. Owens, and R. Pollard. 1997. Cytomegalovirus retinitis after initiation of highly active antiretroviral therapy. Lancet. 349:1443-5.

242. Jacoby, F., and C. R. Leeson. 1959. The post-natal development of the rat submaxillary gland. Journal of anatomy. 93:201-219.

243. Jaffe, H. W., D. J. Bregman, and R. M. Selik. 1983. Acquired immune deficiency syndrome in the United States: the first 1,000 cases. J Infect Dis. 148:339-45.

244. Jones, A. C., P. D. Freedman, J. A. Phelan, R. A. Baughman, and S. M. Kerpel. 1993. Cytomegalovirus infections of the oral cavity. A report of six cases and review of the literature. Oral Surg Oral Med Oral Pathol. 75:76-85. 
245. Jones, R. N., M. L. Neale, B. Beattie, D. Westmoreland, and J. D. Fox. 2000. Development and application of a PCR-based method including an internal control for diagnosis of congenital cytomegalovirus infection. J Clin Microbiol. 38:1-6.

246. Jones, T. R., and L. Sun. 1997. Human cytomegalovirus US2 destabilizes major histocompatibility complex class I heavy chains. J Virol. 71:2970-9.

247. Jones, T. R., E. J. Wiertz, L. Sun, K. N. Fish, J. A. Nelson, and H. L. Ploegh. 1996. Human cytomegalovirus US3 impairs transport and maturation of major histocompatibility complex class I heavy chains. Proc Natl Acad Sci U S A. 93:11327-33.

248. Jonjic, S., W. Mutter, F. Weiland, M. J. Reddehase, and U. H. Koszinowski. 1989. Site-restricted persistent cytomegalovirus infection after selective long-term depletion of CD4+ T lymphocytes. J Exp Med. 169:1199-212.

249. Jonjic, S., I. Pavic, P. Lucin, D. Rukavina, and U. H. Koszinowski. 1990. Efficacious control of cytomegalovirus infection after long-term depletion of CD8+ T lymphocytes. J Virol. 64:5457-64.

250. Jonjic, S., I. Pavic, B. Polic, I. Crnkovic, P. Lucin, and U. H. Koszinowski. 1994. Antibodies are not essential for the resolution of primary cytomegalovirus infection but limit dissemination of recurrent virus. J Exp Med. 179:1713-1717.

251. Jordan, M. C., W. T. Rousseau, J. A. Stewart, G. R. Noble, and T. D. I. Chin. 1973. Spontaneous cytomegalovirus mononucleosis. Clinical and laboratory observations in nine cases. Ann Intern Med. 79:153-60.

252. Kadiiska, M. B., M. J. Burkitt, Q. H. Xiang, and R. P. Mason. 1995. Iron supplementation generates hydroxyl radical in vivo. An ESR spin-trapping investigation. J Clin Invest. 96:1653-7.

253. Kahan, B. D., and P. Troncoso. 1998. Introduction to solid organ transplantation, p. 13-19. In R. A. Bowden and P. Ljungman and C. V. Paya (ed.), Transplant infections. Lippencott-Raven Philadelphia, New York.

254. Kalayjian, R. C., M. L. Cohen, R. A. Bonomo, and T. P. Flanigan. 1993. Cytomegalovirus ventriculoencephalitis in AIDS. A syndrome with distinct clinical and pathologic features. Medicine Baltimore. 72:67-77.

255. Kangro, H. O., H. K. Osman, Y. L. Lau, R. B. Heath, C. Y. Yeung, and M. H. Ng. 1994. Seroprevalence of antibodies to human herpesviruses in England and Hong Kong. J Med Virol. 43:91-6.

256. Karavellas, M. P., C. Y. Lowder, C. Macdonald, C. P. Avila, Jr., and W. R. Freeman. 1998. Immune recovery vitritis associated with inactive cytomegalovirus retinitis: a new syndrome. Arch Ophthalmol. 116:169-75. 
257. Karavellas, M. P., D. J. Plummer, J. C. Macdonald, F. J. Torriani, C. L. Shufelt, S. P. Azen, and W. R. Freeman. 1999. Incidence of immune recovery vitritis in cytomegalovirus retinitis patients following institution of successful highly active antiretroviral therapy. J Infect Dis. 179:697-700.

258. Kaufman, H. S., A. C. Kahn, C. lacobuzio Donahue, M. A. Talamini, K. D. Lillemoe, and S. R. Hamilton. 1999. Cytomegaloviral enterocolitis: clinical associations and outcome. Dis Colon Rectum. 42:24-30.

259. Kauppinen, H., A. Soots, L. Krogerus, T. Brummer, J. Ahonen, and I. Lautenschlager. 1997. Different expression of adhesion molecules ICAM-1 and VCAM-1 and activation markers MHC class II and IL-2R in acute and chronic rejection of rat kidney allografts. Transplant Proc. 29:3150-1.

260. Keay, S., and B. Baldwin. 1992. The human fibroblast receptor for gp86 of human cytomegalovirus is a phosphorylated glycoprotein. J Virol. 66:4834-8.

261. Kendle, J. B., and P. Fan Havard. 1998. Cidofovir in the treatment of cytomegaloviral disease. Ann Pharmacother. 32:1181-92.

262. Kern, F., S. Ode-Hakim, H. Nugel, K. Vogt, H. D. Volk, and P. Reinke. 1996. Peripheral $\mathrm{T}$ cell activation in long-term renal transplant patients: concordant upregulation of adhesion molecules and cytokine gene transcription. J Am Soc Nephrol. 7:2476-82.

263. Kirsch, L. S., J. F. Arevalo, E. De Clercq, E. Chavez de la Paz, D. Munguia, R. Garcia, and W. R. Freeman. 1995. Phase I/II study of intravitreal cidofovir for the treatment of cytomegalovirus retinitis in patients with the acquired immunodeficiency syndrome. Am J Ophthalmol. 119:466-76.

264. Kleijnen, M. F., J. B. Huppa, P. Lucin, S. Mukherjee, H. Farrell, A. E. Campbell, U. H. Koszinowski, A. B. Hill, and H. L. Ploegh. 1997. A mouse cytomegalovirus glycoprotein, gp34, forms a complex with folded class I MHC molecules in the ER which is not retained but is transported to the cell surface. Embo J. 16:685-94.

265. Klemola, E. 1973. Cytomegalovirus infection in previously healthy adults. Ann Intern Med. 79:267-8.

266. Kletzmayr, J., H. Kotzmann, T. Popow-Kraupp, J. Kovarik, and R. Klauser. 1996. Impact of high-dose oral acyclovir prophylaxis on cytomegalovirus (CMV) disease in CMV high-risk renal transplant recipients. J Am Soc Nephrol. 7:325-30.

267. Kloover, J. S., J. L. Hillebrands, G. de Wit, G. Grauls, J. Rozing, C. A. Bruggeman, and P. Nieuwenhuis. 2000. Rat cytomegalovirus replication in the salivary glands is exclusively confined to striated duct cells. Virchows Arch. 437:413-421.

268. Kloover, J. S., M. Scholz, J. Cinatl, Jr., I. Lautenschlager, G. E. Grauls, and C. A. Bruggeman. 1999. Effect of desferrioxamine (DFO) and calcium trinatrium diethylenetriaminepentaacetic acid (DTPA) on rat cytomegalovirus replication in vitro and in vivo. Antiviral Res. 44:55-65. 
269. Klotman, M. E., S. C. Henry, R. C. Greene, P. C. Brazy, P. E. Klotman, and J. D. Hamilton. 1990. Detection of mouse cytomegalovirus nucleic acid in latently infected mice by in vitro enzymatic amplification. J Infect Dis. 161:220-5.

270. Koffron, A. J., M. Hummel, B. K. Patterson, S. Yan, D. B. Kaufman, J. P. Fryer, F. P. Stuart, and M. I. Abecassis. 1998. Cellular localization of latent murine cytomegalovirus. J Virol. 72:95-103.

271. Kondo, K., H. Kaneshima, and E. S. Mocarski. 1994. Human cytomegalovirus latent infection of granulocyte-macrophage progenitors. Proc Natl Acad Sci U S A. 91:11879-83.

272. Kondo, K., and E. S. Mocarski. 1995. Cytomegalovirus latency and latency-specific transcription in hematopoietic progenitors. Scand J Infect Dis Suppl. 99:63-7.

273. Kondo, K., J. Xu, and E. S. Mocarski. 1996. Human cytomegalovirus latent gene expression in granulocyte-macrophage progenitors in culture and in seropositive individuals. Proc Natl Acad Sci U S A. 93:11137-42.

274. Koskinen, P., K. Lemstrom, C. Bruggeman, I. Lautenschlager, and P. Hayry. 1994. Acute cytomegalovirus infection induces a subendothelial inflammation (endothelialitis) in the allograft vascular wall. A possible linkage with enhanced allograft arteriosclerosis. Am J Pathol. 144:41-50.

275. Koskinen, P. K. 1993. The association of the induction of vascular cell adhesion molecule-1 with cytomegalovirus antigenemia in human heart allografts. Transplantation. 56:1103-8.

276. Koskinen, P. K., E. A. Kallio, C. A. Bruggeman, and K. B. Lemstrom. 1997. Cytomegalovirus infection enhances experimental obliterative bronchiolitis in rat tracheal allografts. Am J Respir Crit Care Med. 155:2078-88.

277. Koskinen, P. K., M. S. Nieminen, L. A. Krogerus, K. B. Lemstrom, S. P. Mattila, P. J. Hayry, and I. T. Lautenschlager. 1993. Cytomegalovirus infection and accelerated cardiac allograft vasculopathy in human cardiac allografts. J Heart Lung Transplant. 12:724-9.

278. Koszinowski, U. H., M. Del Val, and M. J. Reddehase. 1990. Cellular and molecular basis of the protective immune response to cytomegalovirus infection. Curr Top Microbiol Immunol. 154:189-220.

279. Krause, H., H. Hebart, G. Jahn, C. A. Muller, and H. Einsele. 1997. Screening for CMV-specific $\mathrm{T}$ cell proliferation to identify patients at risk of developing late onset CMV disease. Bone Marrow Transplant. 19:1111-6.

280. Krech, U. 1973. Complement-fixing antibodies against cytomegalovirus in different parts of the world. Bull World Health Organ. 49:103-6. 
281. Kroshus, T. J., V. R. Kshettry, K. Savik, R. John, M. I. Hertz, and R. M. Bolman, 3rd. 1997. Risk factors for the development of bronchiolitis obliterans syndrome after lung transplantation. J Thorac Cardiovasc Surg. 114:195-202.

282. Kubota, A., S. Kubota, H. E. Farrell, N. Davis-Poynter, and F. Takei. 1999. Inhibition of NK cells by murine CMV-encoded class I MHC homolog m144. Cell Immunol. 191:145-51.

283. Kurz, S., H. P. Steffens, A. Mayer, J. R. Harris, and M. J. Reddehase. 1997. Latency versus persistence or intermittent recurrences: evidence for a latent state of murine cytomegalovirus in the lungs. J Virol. 71:2980-7.

284. Kurz, S. K., M. Rapp, H. P. Steffens, N. K. Grzimek, S. Schmalz, and M. J. Reddehase. 1999. Focal transcriptional activity of murine cytomegalovirus during latency in the lungs. J Virol. 73:482-94.

285. Kurz, S. K., and M. J. Reddehase. 1999. Patchwork pattern of transcriptional reactivation in the lungs indicates sequential checkpoints in the transition from murine cytomegalovirus latency to recurrence. J Virol. 73:8612-22.

286. Kyriazopoulou, V., J. Bondis, F. Frantzidou, A. Athanasiadis, E. Diza, M. Simitsopoulou, and E. Souliou. 1996. Prenatal diagnosis of fetal cytomegalovirus infection in seropositive pregnant women. Eur J Obstet Gynecol Reprod Biol. 69:91-5.

287. Lalezari, J. P., W. L. Drew, E. Glutzer, C. James, D. Miner, J. Flaherty, P. E. Fisher, K. Cundy, J. Hannigan, J. C. Martin, and et al. 1995. (S)-1-[3-hydroxy-2(phosphonylmethoxy)propyl]cytosine (cidofovir): results of a phase I/II study of a novel antiviral nucleotide analogue. J Infect Dis. 171:788-96.

288. Lalezari, J. P., G. N. Holland, F. Kramer, G. F. McKinley, C. A. Kemper, D. V. Ives, R. Nelson, W. D. Hardy, B. D. Kuppermann, D. W. Northfelt, M. Youle, M. Johnson, R. A. Lewis, D. V. Weinberg, G. L. Simon, R. A. Wolitz, A. E. Ruby, R. J. Stagg, and H. S. Jaffe. 1998. Randomized, controlled study of the safety and efficacy of intravenous cidofovir for the treatment of relapsing cytomegalovirus retinitis in patients with AIDS. J Acquir Immune Defic Syndr Hum Retrovirol. 17:339-44.

289. Landini, M. P., T. Lazzarotto, J. Xu, A. P. Geballe, and E. S. Mocarski. 2000. Humoral immune response to proteins of human cytomegalovirus latency-associated transcripts. Biol Blood Marrow Transplant. 6:100-8.

290. Larsen, S. L., L. O. Pedersen, S. Buus, and A. Stryhn. 1996. T cell responses affected by aminopeptidase $\mathrm{N}$ (CD13)-mediated trimming of major histocompatibility complex class II-bound peptides. J Exp Med. 184:183-9.

291. Larsson, S., C. Soderberg-Naucler, F. Z. Wang, and E. Moller. 1998. Cytomegalovirus DNA can be detected in peripheral blood mononuclear cells from all seropositive and most seronegative healthy blood donors over time. Transfusion. 38:2718. 
292. Lautenschlager, I., K. Hockerstedt, H. Jalanko, R. Loginov, K. Salmela, E. Taskinen, and J. Ahonen. 1997. Persistent cytomegalovirus in liver allografts with chronic rejection. Hepatology. 25:190-4.

293. Lautenschlager, I., A. Soots, L. Krogerus, K. Inkinen, J. Kloover, R. Loginov, K. Holma, H. Kauppinen, C. Bruggeman, and J. Ahonen. 1999. Time-related effects of cytomegalovirus infection on the development of chronic renal allograft rejection in a rat model. Intervirology. 42:279-84.

294. Lautenschlager, I., A. Soots, L. Krogerus, H. Kauppinen, O. Saarinen, C. Bruggeman, and J. Ahonen. 1997. Effect of cytomegalovirus on an experimental model of chronic renal allograft rejection under triple-drug treatment in the rat. Transplantation. 64:391-8.

295. Lazetic, S., C. Chang, J. P. Houchins, L. L. Lanier, and J. H. Phillips. 1996. Human natural killer cell receptors involved in $\mathrm{MHC}$ class I recognition are disulfide-linked heterodimers of CD94 and NKG2 subunits. J Immunol. 157:4741-5.

296. Lazzarotto, T., S. Varani, L. Gabrielli, P. Spezzacatena, and M. P. Landini. 1999. New advances in the diagnosis of congenital cytomegalovirus infection. Intervirology. 42:390-7.

297. Lazzarotto, T., S. Varani, B. Guerra, A. Nicolosi, M. Lanari, and M. P. Landini. 2000. Prenatal indicators of congenital cytomegalovirus infection. J Pediatr. 137:90-5.

298. Lazzarotto, T., S. Varani, P. Spezzacatena, P. Pradelli, L. Potena, A. Lombardi, V. Ghisetti, L. Gabrielli, D. A. Abate, C. Magelli, and M. P. Landini. 1998. Delayed acquisition of high-avidity anti-cytomegalovirus antibody is correlated with prolonged antigenemia in solid organ transplant recipients. J Infect Dis. 178:1145-1149.

299. Lea, A. P., and H. M. Bryson. 1996. Cidofovir. Drugs. 52:225-230.

300. Lee, S. 1967. An improved technique of renal transplantation in the rat. Surgery. 61:771-3.

301. Lee, V., I. Subak Sharpe, S. Shah, C. Aitken, S. Limb, and A. Pinching. 1999. Changing trends in cytomegalovirus retinitis with triple therapy. Eye. 13:59-64.

302. Lemstrom, K., P. Koskinen, L. Krogerus, M. Daemen, C. Bruggeman, and P. Hayry. 1995. Cytomegalovirus antigen expression, endothelial cell proliferation, and intimal thickening in rat cardiac allografts after cytomegalovirus infection. Circulation. 92:2594604.

303. Lemstrom, K. B., J. H. Bruning, C. A. Bruggeman, P. K. Koskinen, P. T. Aho, S. Yilmaz, I. T. Lautenschlager, and P. J. Hayry. 1994. Cytomegalovirus infection-enhanced allograft arteriosclerosis is prevented by DHPG prophylaxis in the rat. Circulation. 90:196978. 
304. Lemstrom, K. B., J. H. Bruning, C. A. Bruggeman, I. T. Lautenschlager, and P. J. Hayry. 1993. Cytomegalovirus infection enhances smooth muscle cell proliferation and intimal thickening of rat aortic allografts. J Clin Invest. 92:549-58.

305. Lemstrom, K. B., J. H. Bruning, C. A. Bruggeman, I. T. Lautenschlager, and P. J. Hayry. 1994. Triple drug immunosuppression significantly reduces immune activation and allograft arteriosclerosis in cytomegalovirus-infected rat aortic allografts and induces early latency of viral infection. Am J Pathol. 144:1334-47.

306. Leong, C. C., T. L. Chapman, P. J. Bjorkman, D. Formankova, E. S. Mocarski, J. H. Phillips, and L. L. Lanier. 1998. Modulation of natural killer cell cytotoxicity in human cytomegalovirus infection: the role of endogenous class I major histocompatibility complex and a viral class I homolog. J Exp Med. 187:1681-7.

307. Leport, C., S. Puget, J. M. Pepin, S. Levy, C. Perronne, F. Brun Vezinet, and J. L. Vilde. 1993. Cytomegalovirus resistant to foscarnet: clinicovirologic correlation in a patient with human immunodeficiency virus [letter]. J Infect Dis. 168:1329-30.

308. Li, C. R., P. D. Greenberg, M. J. Gilbert, J. M. Goodrich, and S. R. Riddell. 1994. Recovery of HLA-restricted cytomegalovirus (CMV)-specific T-cell responses after allogeneic bone marrow transplant: correlation with CMV disease and effect of ganciclovir prophylaxis. Blood. 83:1971-9.

309. Li, F., M. Yin, J. G. Van Dam, G. Grauls, J. Rozing, and C. A. Bruggeman. 1998. Cytomegalovirus infection enhances the neointima formation in rat aortic allografts: effect of major histocompatibility complex class I and class II antigen differences. Transplantation. 65:1298-304.

310. Li, F. L., G. Grauls, M. Yin, and C. A. Bruggeman. 1996. Correlation between the intensity of cytomegalovirus infection and the amount of perivasculitis in aortic allografts. Transpl Int. 9 Suppl 1:S340-4.

311. Libby, P., D. Egan, and S. Skarlatos. 1997. Roles of infectious agents in atherosclerosis and restenosis: an assessment of the evidence and need for future research. Circulation. 96:4095-103.

312. Liesnard, C., C. Donner, F. Brancart, F. Gosselin, M. L. Delforge, and F. Rodesch. 2000. Prenatal diagnosis of congenital cytomegalovirus infection: prospective study of 237 pregnancies at risk. Obstet Gynecol. 95:881-8.

313. Liles, J. H., P. A. Flecknell, J. Roughan, and I. Cruz Madorran. 1998. Influence of oral buprenorphine, oral naltrexone or morphine on the effects of laparotomy in the rat. Lab Anim. 32:149-161.

314. Linnemann, C. C., Jr., C. A. Kauffman, M. R. First, G. M. Schiff, and J. P. Phair. 1978. Cellular immune response to cytomegalovirus infection after renal transplantation. Infect Immun. 22:176-80. 
315. Littler, E., A. D. Stuart, and M. S. Chee. 1992. Human cytomegalovirus UL97 open reading frame encodes a protein that phosphorylates the antiviral nucleoside analogue ganciclovir. Nature. 358:160-2.

316. Ljunggren, H. G., and K. Karre. 1990. In search of the 'missing self: MHC molecules and NK cell recognition. Immunol Today. 11:237-44.

317. Ljungman, P., J. Aschan, I. Lewensohn-Fuchs, S. Carlens, K. Larsson, B. Lonnqvist, J. Mattsson, E. Sparrelid, J. Winiarski, and O. Ringden. 1998. Results of different strategies for reducing cytomegalovirus-associated mortality in allogeneic stem cell transplant recipients. Transplantation. 66:1330-4.

318. Ljungman, P., G. Oberg, J. Aschan, A. Ehrnst, B. Lonnqvist, K. Pauksen, and P. Sulila. 1996. Foscarnet for pre-emptive therapy of CMV infection detected by a leukocytebased nested PCR in allogeneic bone marrow transplant patients. Bone Marrow Transplant. 18:565-8.

319. Loebe, M., S. Schuler, O. Zais, H. Warnecke, E. Fleck, and R. Hetzer. 1990. Role of cytomegalovirus infection in the development of coronary artery disease in the transplanted heart. J Heart Transplant. 9:707-11.

320. Lowance, D., H. H. Neumayer, C. M. Legendre, J. P. Squifflet, J. Kovarik, P. J. Brennan, D. Norman, R. Mendez, M. R. Keating, G. L. Coggon, A. Crisp, and I. C. Lee. 1999. Valacyclovir for the prevention of cytomegalovirus disease after renal transplantation. International Valacyclovir Cytomegalovirus Prophylaxis Transplantation Study Group. N Engl J Med. 340:1462-70.

321. Lucin, P., I. Pavic, B. Polic, S. Jonjic, and U. H. Koszinowski. 1992. Gamma interferon-dependent clearance of cytomegalovirus infection in salivary glands. J Virol. 66:1977-84.

322. Lunetta, J. M., and J. A. Wiedeman. 2000. Latency-associated sense transcripts are expressed during in vitro human cytomegalovirus productive infection. Virology. 278:467-76.

323. MacCormac, L. P., and J. E. Grundy. 1999. Two clinical isolates and the Toledo strain of cytomegalovirus contain endothelial cell tropic variants that are not present in the AD169, Towne, or Davis strains. J Med Virol. 57:298-307.

324. Mackay, C. R., and B. A. Imhof. 1993. Cell adhesion in the immune system. Immunol Today. 14:99-102.

325. Madrigal, J. A., R. Arguello, I. Scott, and H. Avakian. 1997. Molecular histocompatibility typing in unrelated donor bone marrow transplantation. Blood Rev. 11:105-17.

326. Manning, W. C., C. A. Stoddart, L. A. Lagenaur, G. B. Abenes, and E. S. Mocarski. 1992. Cytomegalovirus determinant of replication in salivary glands. J Virol. 66:3794-3802. 
327. Marks, D. I., J. O. Cullis, K. N. Ward, S. Lacey, R. Syzdlo, T. P. Hughes, A. P. Schwarer, E. Lutz, A. J. Barrett, J. M. Hows, and et al. 1993. Allogeneic bone marrow transplantation for chronic myeloid leukemia using sibling and volunteer unrelated donors. A comparison of complications in the first 2 years. Ann Intern Med. 119:207-14.

328. Marshall, G. S., G. P. Rabalais, J. A. Stewart, and J. G. Dobbins. 1993. Cytomegalovirus seroprevalence in women bearing children in Jefferson County, Kentucky. Am J Med Sci. 305:292-6.

329. Martelius, T., L. Krogerus, K. Hockerstedt, C. Bruggeman, and I. Lautenschlager. 1998. Cytomegalovirus infection is associated with increased inflammation and severe bile duct damage in rat liver allografts. Hepatology. 27:996-1002.

330. Martelius, T., H. Makisalo, K. Hockerstedt, E. Taskinen, and I. Lautenschlager. 1997. A rat model of monitoring liver allograft rejection. Transpl Int. 10:103-8.

331. Martelius, T., M. Scholz, L. Krogerus, K. Hockerstedt, R. Loginov, C. Bruggeman, J. Cinatl, Jr., H. W. Doerr, and I. Lautenschlager. 1999. Antiviral and immunomodulatory effects of desferrioxamine in cytomegalovirus-infected rat liver allografts with rejection. Transplantation. 68:1753-61.

332. Martin, D. F., J. P. Dunn, J. L. Davis, J. S. Duker, R. E. Engstrom, Jr., D. N. Friedberg, G. J. Jaffe, B. D. Kuppermann, M. A. Polis, R. J. Whitley, R. A. Wolitz, and C. A. Benson. 1999. Use of the ganciclovir implant for the treatment of cytomegalovirus retinitis in the era of potent antiretroviral therapy: recommendations of the International AIDS Society-USA panel. Am J Ophthalmol. 127:329-39.

333. Martin, D. F., B. D. Kuppermann, R. A. Wolitz, A. G. Palestine, H. Li, and C. A. Robinson. 1999. Oral ganciclovir for patients with cytomegalovirus retinitis treated with a ganciclovir implant. Roche Ganciclovir Study Group. N Engl J Med. 340:1063-70.

334. Martin, D. F., D. J. Parks, S. D. Mellow, F. L. Ferris, R. C. Walton, N. A. Remaley, E. Y. Chew, P. Ashton, M. D. Davis, and R. B. Nussenblatt. 1994. Treatment of cytomegalovirus retinitis with an intraocular sustained-release ganciclovir implant. A randomized controlled clinical trial. Arch Ophthalmol. 112:1531-9.

335. Masuda, J., and R. Ross. 1990. Atherogenesis during low level hypercholesterolemia in the nonhuman primate. I. Fatty streak formation. Arteriosclerosis. 10:164-77.

336. Masuda, J., and R. Ross. 1990. Atherogenesis during low level hypercholesterolemia in the nonhuman primate. II. Fatty streak conversion to fibrous plaque. Arteriosclerosis. 10:178-87.

337. McCormack, J. G., S. D. Bowler, J. E. Donnelly, and C. Steadman. 1998. Successful treatment of severe cytomegalovirus infection with ganciclovir in an immunocompetent host. Clin Infect Dis. 26:1007-8. 
338. McCutchan, J. A. 1995. Clinical impact of cytomegalovirus infections of the nervous system in patients with AIDS. Clin Infect Dis. 21 Suppl 2:S196-201.

339. McDonald, K., T. S. Rector, E. A. Braulin, S. H. Kubo, and M. T. Olivari. 1989. Association of coronary artery disease in cardiac transplant recipients with cytomegalovirus infection. Am J Cardiol. 64:359-62.

340. McGeoch, D. J., S. Cook, A. Dolan, F. E. Jamieson, and E. A. Telford. 1995. Molecular phylogeny and evolutionary timescale for the family of mammalian herpesviruses. J Mol Biol. 247:443-58.

341. McGill, H. C., Jr. 1984. George Lyman Duff memorial lecture. Persistent problems in the pathogenesis of atherosclerosis. Arteriosclerosis. 4:443-51.

342. McKenzie, R., W. D. Travis, S. A. Dolan, S. Pittaluga, I. M. Feuerstein, J. Shelhamer, R. Yarchoan, and H. Masur. 1991. The causes of death in patients with human immunodeficiency virus infection: a clinical and pathologic study with emphasis on the role of pulmonary diseases. Medicine Baltimore. 70:326-43.

343. Meijer, H., J. C. Dreesen, and C. P. Van Boven. 1986. Molecular cloning and restriction endonuclease mapping of the rat cytomegalovirus genome. J Gen Virol. 67:1327-42.

344. Melnick, J. L., C. Hu, J. Burek, E. Adam, and M. E. DeBakey. 1994. Cytomegalovirus DNA in arterial walls of patients with atherosclerosis. J Med Virol. 42:170-4.

345. Melnick, J. L., B. L. Petrie, G. R. Dreesman, J. Burek, C. H. McCollum, and M. E. DeBakey. 1983. Cytomegalovirus antigen within human arterial smooth muscle cells. Lancet. 2:644-7.

346. Mendelson, M., S. Monard, P. Sissons, and J. Sinclair. 1996. Detection of endogenous human cytomegalovirus in CD34+ bone marrow progenitors. J Gen Virol. 77:3099-102.

347. Mercer, J. A., C. A. Wiley, and D. H. Spector. 1988. Pathogenesis of murine cytomegalovirus infection: identification of infected cells in the spleen during acute and latent infections. J Virol. 62:987-97.

348. Meyers, J. D., N. Flournoy, and E. D. Thomas. 1982. Nonbacterial pneumonia after allogeneic marrow transplantation: a review of ten years' experience. Rev Infect Dis. 4:1119-32.

349. Meyers, J. D., N. Flournoy, and E. D. Thomas. 1986. Risk factors for cytomegalovirus infection after human marrow transplantation. J Infect Dis. 153:478-88.

350. Meyers, J. D., P. Ljungman, and L. D. Fisher. 1990. Cytomegalovirus excretion as a predictor of cytomegalovirus disease after marrow transplantation: importance of cytomegalovirus viremia. J Infect Dis. 162:373-80. 
351. Meyers, J. D., E. C. Reed, D. H. Shepp, M. Thornquist, P. S. Dandliker, C. A. Vicary, N. Flournoy, L. E. Kirk, J. H. Kersey, E. D. Thomas, and et al. 1988. Acyclovir for prevention of cytomegalovirus infection and disease after allogeneic marrow transplantation. N Engl J Med. 318:70-5.

352. Michelet, C., C. Arvieux, C. Francois, J. M. Besnier, J. P. Rogez, J. P. Breux, F. Souala, C. Allavena, F. Raffi, M. Garre, and F. Cartier. 1998. Opportunistic infections occurring during highly active antiretroviral treatment. Aids. 12:1815-22.

353. Michelson, S. 1999. Human cytomegalovirus escape from immune detection. Intervirology. 42:301-7.

354. Miller, D. M., B. M. Rahill, J. M. Boss, M. D. Lairmore, J. E. Durbin, J. W. Waldman, and D. D. Sedmak. 1998. Human cytomegalovirus inhibits major histocompatibility complex class II expression by disruption of the Jak/Stat pathway. J Exp Med. 187:675-83.

355. Miller, W., P. Flynn, J. McCullough, H. H. Balfour, Jr., A. Goldman, R. Haake, P. McGlave, N. Ramsay, and J. Kersey. 1986. Cytomegalovirus infection after bone marrow transplantation: an association with acute graft-v-host disease. Blood. 67:1162-7.

356. Mims, C. A., and J. Gould. 1979. Infection of salivary glands, kidneys, adrenals, ovaries and epithelia by murine cytomegalovirus. J Med Microbiol. 12:113-22.

357. Minton, E. J., C. Tysoe, J. H. Sinclair, and J. G. Sissons. 1994. Human cytomegalovirus infection of the monocyte/macrophage lineage in bone marrow. J Virol. 68:4017-21.

358. Mitchell, B. M., A. Leung, and J. G. Stevens. 1996. Murine cytomegalovirus DNA in peripheral blood of latently infected mice is detectable only in monocytes and polymorphonuclear leukocytes. Virology. 223:198-207.

359. Mocarski, E. S. j. 1996. Herpesviridae, p. 2447-2492. In B. N. Fields and D. M. Knipe and P. M. Howley (ed.), Fields Virology, vol. 3. Lippincott-Raven Publishers, Philadelphia.

360. Morgello, S., E. S. Cho, S. Nielsen, O. Devinsky, and C. K. Petito. 1987. Cytomegalovirus encephalitis in patients with acquired immunodeficiency syndrome: an autopsy study of 30 cases and a review of the literature. Hum Pathol. 18:289-97.

361. Mosca, J. D., D. P. Bednarik, N. B. Raj, C. A. Rosen, J. G. Sodroski, W. A. Haseltine, G. S. Hayward, and P. M. Pitha. 1987. Activation of human immunodeficiency virus by herpesvirus infection: identification of a region within the long terminal repeat that responds to a trans-acting factor encoded by herpes simplex virus 1. Proc Natl Acad Sci U S A. $84: 7408-12$.

362. Mouritsen, S., M. Meldal, O. Werdelin, A. S. Hansen, and S. Buus. 1992. MHC molecules protect T cell epitopes against proteolytic destruction. J Immunol. 149:1987-93. 
363. Murph, J. R., J. C. Baron, C. K. Brown, C. L. Ebelhack, and J. F. Bale, Jr. 1991. The occupational risk of cytomegalovirus infection among day-care providers. Jama. 265:603-8.

364. Murph, J. R., I. E. Souza, J. D. Dawson, P. Benson, S. J. Petheram, D. Pfab, A. Gregg, M. E. O'Neill, B. Zimmerman, and J. F. Bale, Jr. 1998. Epidemiology of congenital cytomegalovirus infection: maternal risk factors and molecular analysis of cytomegalovirus strains. Am J Epidemiol. 147:940-7.

365. Musch, D. C., D. F. Martin, J. F. Gordon, M. D. Davis, and B. D. Kuppermann. 1997. Treatment of cytomegalovirus retinitis with a sustained-release ganciclovir implant. The Ganciclovir Implant Study Group. N Engl J Med. 337:83-90.

366. Myers, J. D., H. C. Spencer, Jr., J. C. Watts, M. B. Gregg, J. A. Stewart, R. H. Troupin, and E. D. Thomas. 1975. Cytomegalovirus pneumonia after human marrow transplantation. Ann Intern Med. 82:181-8.

367. Nanda, R., and F. A. Catalanotto. 1981. Long-term effects of surgical desalivation upon taste acuity, fluid intake, and taste buds in the rat. J Dent Res. 60:69-76.

368. Natali, A., P. Valcavi, M. C. Medici, E. Dieci, S. Montali, and C. Chezzi. 1997. Cytomegalovirus infection in an Italian population: antibody prevalence, virus excretion and maternal transmission. New Microbiol. 20:123-133.

369. Nelson, C. T., and G. J. Demmler. 1997. Cytomegalovirus infection in the pregnant mother, fetus, and newborn infant. Clin Perinatol. 24:151-60.

370. Nelson, J. A., C. Reynolds Kohler, M. B. Oldstone, and C. A. Wiley. 1988. HIV and HCMV coinfect brain cells in patients with AIDS. Virology. 165:286-90.

371. Neyts, J., J. Balzarini, L. Naesens, and E. De Clercq. 1992. Efficacy of (s)-1-(3hydroxy-2-phosphonylmethoxypropyl)cytosine and 9-(1,3-dihydroxy-2propoxymethyl)guanine for the treatment of murine cytomegalovirus infection in severe combined immunodeficiency mice. J Med Virol. 37:67-71.

372. Neyts, J., R. Snoeck, J. Balzarini, and E. De Clercq. 1991. Particular characteristics of the anti-human cytomegalovirus activity of (S)-1-(3-hydroxy-2phosphonylmethoxypropyl)cytosine (HPMPC) in vitro. Antiviral Res. 16:41-52.

373. Neyts, J., R. Snoeck, D. Schols, J. Balzarini, and E. De Clercq. 1990. Selective inhibition of human cytomegalovirus DNA synthesis by (S)-1-(3-hydroxy-2phosphonylmethoxypropyl)cytosine [(S)-HPMPC] and 9-(1,3-dihydroxy-2propoxymethyl)guanine (DHPG). Virology. 179:41-50.

374. Neyts, J., and F. S. Stals. 1993. Efficacy of HPMPC in the treatment of CMV infections in various animal models. ???:279-285.

375. Nichols, W. G., and M. Boeckh. 2000. Recent advances in the therapy and prevention of CMV infections. J Clin Virol. 16:25-40. 
376. Nicholson, T. M., and N. E. Rowland. 1994. Effect of desalivation on water and sodium chloride intake in rats. Physiol Behav. 56:543-547.

377. Nishimura, N., H. Kimura, Y. Yabuta, N. Tanaka, Y. Ito, K. Ishikawa, C. Suzuki, and T. Morishima. 1999. Prevalence of maternal cytomegalovirus (CMV) antibody and detection of CMV DNA in amniotic fluid. Microbiol Immunol. 43:781-784.

378. Noble, S., and D. Faulds. 1998. Ganciclovir. An update of its use in the prevention of cytomegalovirus infection and disease in transplant recipients. Drugs. 56:115-46.

379. O'Grady, J., G. J. Alexander, S. Sutherland, P. T. Donaldson, F. Harvey, B. Portmann, R. Y. Calne, and R. Williams. 1988. Cytomegalovirus infection and donor/recipient HLA antigens: interdependent co-factors in pathogenesis of vanishing bileduct syndrome after liver transplantation. Lancet. 2:302-5.

380. O'Grady, J. G., G. J. Alexander, S. Sutherland, P. T. Donaldson, F. Harvey, B. Portmann, R. Y. Calne, and R. Williams. 1988. Cytomegalovirus infection and donor/recipient HLA antigens: interdependent co-factors in pathogenesis of vanishing bileduct syndrome after liver transplantation. Lancet. 2:302-5.

381. Orange, J. S., and C. A. Biron. 1996. Characterization of early IL-12, IFNalphabeta, and TNF effects on antiviral state and NK cell responses during murine cytomegalovirus infection. J Immunol. 156:4746-56.

382. Osborn, J. E. 1982. CMV-herpesviruses of mice, p. 267-272. In H. L. Foster and J. G. Fox and J. D. Small (ed.), The mouse in biomedical research, vol. 2. Academic press, Inc., New York.

383. Palella, F. J., Jr., K. M. Delaney, A. C. Moorman, M. O. Loveless, J. Fuhrer, G. A. Satten, D. J. Aschman, and S. D. Holmberg. 1998. Declining morbidity and mortality among patients with advanced human immunodeficiency virus infection. HIV Outpatient Study Investigators. N Engl J Med. 338:853-60.

384. Pass, R. F., and C. Hutto. 1986. Group day care and cytomegaloviral infections of mothers and children. Rev Infect Dis. 8:599-605.

385. Pass, R. F., C. Hutto, M. D. Lyon, and G. Cloud. 1990. Increased rate of cytomegalovirus infection among day care center workers. Pediatr Infect Dis J. 9:465-70.

386. Pass, R. F., C. Hutto, R. Ricks, and G. A. Cloud. 1986. Increased rate of cytomegalovirus infection among parents of children attending day-care centers. $\mathrm{N}$ Engl J Med. 314:1414-8.

387. Pass, R. F., S. Stagno, G. J. Myers, and C. A. Alford. 1980. Outcome of symptomatic congenital cytomegalovirus infection: results of long-term longitudinal followup. Pediatrics. 66:758-62.

388. Patel, R., and C. V. Paya. 1998. Cytomegalovirus infection and disease in solid organ transplant recipients, p. 229-244. In R. A. Bowden and P. Ljungman and C. V. Paya (ed.), Transplant infections. Lippencott-Raven Philadelphia, New York. 
389. Patel, R., and C. V. Paya. 1997. Infections in solid-organ transplant recipients. Clin Microbiol Rev. 10:86-124.

390. Paul, L. C. 1995. Chronic renal transplant loss. Kidney Int. 47:1491-9.

391. Paul, L. C. 1994. Functional and histologic characteristics of chronic renal allograft rejection. Clin Transplant. 8:319-23.

392. Paul, L. C., P. Hayry, M. Foegh, M. J. Dennis, M. J. Mihatsch, E. Larsson, and B. Fellstrom. 1993. Diagnostic criteria for chronic rejection/accelerated graft atherosclerosis in heart and kidney transplants: joint proposal from the Fourth Alexis Carrel Conference on Chronic Rejection and Accelerated Arteriosclerosis in Transplanted Organs. Transplant Proc. 25:2022-3.

393. Peckham, C. S. 1991. Cytomegalovirus infection: congenital and neonatal disease. Scand J Infect Dis Suppl. 80:82-7.

394. Pellegrin, I., I. Garrigue, D. Ekouevi, L. Couzi, P. Merville, P. Merel, G. Chene, M. H. Schrive, P. Trimoulet, M. E. Lafon, and H. Fleury. 2000. New molecular assays to predict occurrence of cytomegalovirus disease in renal transplant recipients. J Infect Dis. 182:36-42.

395. Pepperl, S., J. Munster, M. Mach, J. R. Harris, and B. Plachter. 2000. Dense bodies of human cytomegalovirus induce both humoral and cellular immune responses in the absence of viral gene expression. J Virol. 74:6132-46.

396. Perry, C. M., and J. A. Balfour. 1999. Fomivirsen. Drugs. 57:375-80; discussion 381.

397. Persoons, M. C., M. J. Daemen, J. H. Bruning, and C. A. Bruggeman. 1994. Active cytomegalovirus infection of arterial smooth muscle cells in immunocompromised rats. A clue to herpesvirus-associated atherogenesis? Circ Res. 75:214-20.

398. Persoons, M. C., M. J. Daemen, E. M. van Kleef, G. E. Grauls, E. Wijers, and C. A. Bruggeman. 1997. Neointimal smooth muscle cell phenotype is important in its susceptibility to cytomegalovirus (CMV) infection: a study in rat. Cardiovasc Res. 36:2828.

399. Persoons, M. C. J., F. S. Stals, M. C. E. Van Dam Mieras, and C. A. Bruggeman. 1998. Multiple organ involvement during experimental cytomegalovirus infection is associated with disseminated vascular pathology. J PATHOL. 184:103-109.

400. Peters, B. S., E. J. Beck, S. Anderson, D. Coleman, R. Coker, J. Main, C. Migdal, J. R. Harris, and A. J. Pinching. 1991. Cytomegalovirus infection in AIDS. Patterns of disease, response to therapy and trends in survival. J Infect. 23:129-137.

401. Phillips, A. J., P. Tomasec, E. C. Wang, G. W. Wilkinson, and L. K. Borysiewicz. 1998. Human cytomegalovirus infection downregulates expression of the cellular aminopeptidases CD10 and CD13. Virology. 250:350-8. 
402. Pialoux, G., P. Ravisse, P. Trotot, and B. Dupont. 1991. Cytomegalovirus infection of the submandibular gland in a patient with AIDS. Rev Infect Dis. 13:338.

403. Piascik, P. 1999. Fomiversen sodium approved to treat CMV retinitis. J Am Pharm Assoc (Wash). 39:84-5.

404. Pippard, M. J., and S. T. Callender. 1983. The management of iron chelation therapy. Br J Haematol. 54:503-7.

405. Pober, J. S., and R. S. Cotran. 1990. Cytokines and endothelial cell biology. Physiol Rev. 70:427-51.

406. Porter, K. R., D. M. Starnes, and J. D. Hamilton. 1985. Reactivation of latent murine cytomegalovirus from kidney. Kidney Int. 28:922-5.

407. Pouteil Noble, C., R. Ecochard, G. Landrivon, A. Donia Maged, J. C. Tardy, S. Bosshard, S. Colon, H. Betuel, M. Aymard, and J. L. Touraine. 1993. Cytomegalovirus infection--an etiological factor for rejection? A prospective study in 242 renal transplant patients. Transplantation. 55:851-7.

408. Prentice, H. G., E. Gluckman, R. L. Powles, P. Ljungman, N. Milpied, J. M. Fernandez Ranada, F. Mandelli, P. Kho, L. Kennedy, and A. R. Bell. 1994. Impact of longterm acyclovir on cytomegalovirus infection and survival after allogeneic bone marrow transplantation. European Acyclovir for CMV Prophylaxis Study Group. Lancet. 343:74953.

409. Prentice, H. G., E. Gluckman, R. L. Powles, P. Ljungman, N. J. Milpied, R. Camara, F. Mandelli, P. Kho, L. Kennedy, and A. R. Bell. 1997. Long-term survival in allogeneic bone marrow transplant recipients following acyclovir prophylaxis for CMV infection. The European Acyclovir for CMV Prophylaxis Study Group. Bone Marrow Transplant. 19:129-33.

410. Price, P., and S. D. Olver. 1996. Syndromes induced by cytomegalovirus infection. Clin Immunol Immunopathol. 80:215-24.

411. Prosch, S., W. D. Docke, P. Reinke, H. D. Volk, and D. H. Kruger. 1999. Human cytomegalovirus reactivation in bone-marrow-derived granulocyte/monocyte progenitor cells and mature monocytes. Intervirology. 42:308-13.

412. Prosch, S., K. Staak, J. Stein, C. Liebenthal, T. Stamminger, H.-D. Volk, and D. h. Kruger. 1995. Stimulation of the human cytomegalovirus IE enhancer/promotor in HL-60 cells bij TNF-alpha is mediated via induction of NF-kappaB. virology. 208:197-206.

413. Prosch, S., C. E. Wendt, P. Reinke, C. Priemer, M. Oppert, D. H. Kruger, H. D. Volk, and W. D. Docke. 2000. A novel link between stress and human cytomegalovirus (HCMV) infection: sympathetic hyperactivity stimulates HCMV activation. Virology. 272:357-65.

414. Pulakhandam, U., and H. P. Dincsoy. 1990. Cytomegaloviral adrenalitis and adrenal insufficiency in AIDS. Am J Clin Pathol. 93:651-6. 
415. Question-and-answer-session. 1993. Update on CMV infection in solid-organ transplantation'. Transplant proc. 25:27-28.

416. Quinn, T. C., P. Piot, J. B. McCormick, F. M. Feinsod, H. Taelman, B. Kapita, W. Stevens, and A. S. Fauci. 1987. Serologic and immunologic studies in patients with AIDS in North America and Africa. The potential role of infectious agents as cofactors in human immunodeficiency virus infection. Jama. 257:2617-21.

417. Quinnan, G. V., Jr., W. H. Burns, N. Kirmani, A. H. Rook, J. Manischewitz, L. Jackson, G. W. Santos, and R. Saral. 1984. HLA-restricted cytotoxic T lymphocytes are an early immune response and important defense mechanism in cytomegalovirus infections. Rev Infect Dis. 6:156-63.

418. Rawlinson, W. D., H. E. Farrell, and B. G. Barrell. 1996. Analysis of the complete DNA sequence of murine cytomegalovirus. J Virol. 70:8833-49.

419. Reddehase, M. J., M. Balthesen, M. Rapp, S. Jonjic, I. Pavic, and U. H. Koszinowski. 1994. The conditions of primary infection define the load of latent viral genome in organs and the risk of recurrent cytomegalovirus disease. J Exp Med. 179:185193.

420. Reddehase, M. J., and U. H. Koszinowski. 1984. Significance of herpesvirus immediate early gene expression in cellular immunity to cytomegalovirus infection. Nature. 312:369-71.

421. Reddehase, M. J., W. Mutter, K. Munch, H. J. Buhring, and U. H. Koszinowski. 1987. CD8-positive T lymphocytes specific for murine cytomegalovirus immediate-early antigens mediate protective immunity. J Virol. 61:3102-8.

422. Rehbinder, C., P. Baneux, D. Forbes, H. van Herck, W. Nicklas, Z. Rugaya, and G. Winkler. 1996. FELASA recommendations for the health monitoring of mouse, rat, hamster, gerbil, guinea pig and rabbit experimental units. Report of the Federation of European Laboratory Animal Science Associations (FELASA) Working Group on Animal Health accepted by the FELASA Board of Management, November 1995. Lab Anim. 30:193-208.

423. Reinke, P., E. Fietze, S. Ode Hakim, S. Prosch, J. Lippert, R. Ewert, and H. D. Volk. 1994. Late-acute renal allograft rejection and symptomless cytomegalovirus infection. Lancet. 344:1737-8.

424. Reusch, U., W. Muranyi, P. Lucin, H. G. Burgert, H. Hengel, and U. H. Koszinowski. 1999. A cytomegalovirus glycoprotein re-routes MHC class I complexes to lysosomes for degradation. Embo J. 18:1081-91.

425. Reusser, P. 1996. The challenge of cytomegalovirus infection after bone marrow transplantation: epidemiology, prophylaxis, and therapy. Bone Marrow Transplant. 18:1079. 
426. Reusser, P., G. Cathomas, R. Attenhofer, M. Tamm, and G. Thiel. 1999. Cytomegalovirus (CMV)-specific $\mathrm{T}$ cell immunity after renal transplantation mediates protection from CMV disease by limiting the systemic virus load. J Infect Dis. 180:247-53.

427. Reusser, P., J. G. Gambertoglio, K. Lilleby, and J. D. Meyers. 1992. Phase I-II trial of foscarnet for prevention of cytomegalovirus infection in autologous and allogeneic marrow transplant recipients. J Infect Dis. 166:473-9.

428. Reyburn, H. T., O. Mandelboim, M. Vales Gomez, D. M. Davis, L. Pazmany, and J. L. Strominger. 1997. The class I MHC homolog of human cytomegalovirus inhibits attack by natural killer cells. Nature. 386:514-7.

429. Rice, G. P., R. D. Schrier, and M. B. Oldstone. 1984. Cytomegalovirus infects human lymphocytes and monocytes: virus expression is restricted to immediate-early gene products. Proc Natl Acad Sci U S A. 81:6134-8.

430. Richman, D. D., M. A. Fischl, M. H. Grieco, M. S. Gottlieb, P. A. Volberding, O. L. Laskin, J. M. Leedom, J. E. Groopman, D. Mildvan, M. S. Hirsch, and et al. 1987. The toxicity of azidothymidine (AZT) in the treatment of patients with AIDS and AIDS-related complex. A double-blind, placebo-controlled trial. N Engl J Med. 317:192-7.

431. Riddell, S. R., and P. D. Greenberg. 1994. Therapeutic reconstitution of human viral immunity by adoptive transfer of cytotoxic T lymphocyte clones. Curr Top Microbiol Immunol. 189:9-34.

432. Riddell, S. R., K. S. Watanabe, J. M. Goodrich, C. R. Li, M. E. Agha, and P. D. Greenberg. 1992. Restoration of viral immunity in immunodeficient humans by the adoptive transfer of T cell clones. Science. 257:238-241.

433. Ridker, P. M., C. H. Hennekens, M. J. Stampfer, and F. Wang. 1998. Prospective study of herpes simplex virus, cytomegalovirus, and the risk of future myocardial infarction and stroke. Circulation. 98:2796-9.

434. Roizman, B. 1996. Herpesviridae, p. 2221-2230. In B. N. Fields and D. M. Knipe and P. M. Howley (ed.), Fields Virology, vol. 3. Lippincott-Raven Publishers, Philadelphia. 435. Roizman, B., and A. E. Sears. 1987. An inquiry into the mechanisms of herpes simplex virus latency. Annu Rev Microbiol. 41:543-71.

436. Rosenthal, S. L., L. R. Stanberry, F. M. Biro, M. Slaoui, M. Francotte, M. Koutsoukos, M. Hayes, and D. I. Bernstein. 1997. Seroprevalence of herpes simplex virus types 1 and 2 and cytomegalovirus in adolescents. Clin Infect Dis. 24:135-9.

437. Ross, R. 1999. Atherosclerosis is an inflammatory disease. Am Heart J. 138:S419-20.

438. Ross, R. 1999. Atherosclerosis--an inflammatory disease. N Engl J Med. 340:11526.

439. Ross, R. 1993. The pathogenesis of atherosclerosis: a perspective for the 1990s. Nature. 362:801-9. 
440. Rubin, R. H., P. Lynch, M. S. Pasternack, D. Schoenfeld, and D. N. Medearis, Jr. 1989. Combined antibody and ganciclovir treatment of murine cytomegalovirus-infected normal and immunosuppressed BALB/c mice. Antimicrob Agents Chemother. 33:1975-9.

441. Ruellan-Eugene, G., P. Barjot, M. Campet, A. Vabret, M. Herlicoviez, G. Muller, G. Levy, B. Guillois, and F. Freymuth. 1996. Evaluation of virological procedures to detect fetal human cytomegalovirus infection: avidity of IgG antibodies, virus detection in amniotic fluid and maternal serum. J Med Virol. 50:9-15.

442. Salazar, A., D. Podzamczer, R. Rene, M. Santin, J. L. Perez, I. Ferrer, P. Fernandez Viladrich, and F. Gudiol. 1995. Cytomegalovirus ventriculoencephalitis in AIDS patients. Scand J Infect Dis. 27:165-9.

443. Salazar-Mather, T. P., J. S. Orange, and C. A. Biron. 1998. Early murine cytomegalovirus (MCMV) infection induces liver natural killer (NK) cell inflammation and protection through macrophage inflammatory protein 1alpha (MIP-1alpha)-dependent pathways. J Exp Med. 187:1-14.

444. Salzberger, B., R. A. Bowden, R. C. Hackman, C. Davis, and M. Boeckh. 1997. Neutropenia in allogeneic marrow transplant recipients receiving ganciclovir for prevention of cytomegalovirus disease: risk factors and outcome. Blood. 90:2502-8.

445. Sambiase, N. V., M. L. Higuchi, G. Nuovo, P. S. Gutierrez, A. I. Fiorelli, D. E. Uip, and J. A. Ramires. 2000. CMV and transplant-related coronary atherosclerosis: an immunohistochemical, in situ hybridization, and polymerase chain reaction in situ study. Mod Pathol. 13:173-9.

446. Sandford, G. R., and W. H. Burns. 1988. Use of temperature-sensitive mutants of mouse cytomegalovirus as vaccines. J Infect Dis. 158:596-601.

447. Santos, D. V., M. M. Souza, S. H. Goncalves, A. C. Cotta, L. A. Melo, G. M. Andrade, and G. Brasileiro-Filho. 1999. Congenital cytomegalovirus infection in a neonatal intensive care unit in brazil evaluated by PCR and association with perinatal aspects. $J$ Perinat Med. 27:116-21.

448. Sarasini, A., F. Baldanti, M. Furione, E. Percivalle, R. Brerra, M. Barbi, and G. Gerna. 1995. Double resistance to ganciclovir and foscarnet of four human cytomegalovirus strains recovered from AIDS patients. J Med Virol. 47:237-44.

449. Sawyer, M. D., J. L. Mayoral, K. J. Gillingham, M. A. Kramer, and D. L. Dunn. 1993. Treatment of recurrent cytomegalovirus disease in patients receiving solid organ transplants. Arch Surg. 128:165-9; discussion 170.

450. Scholz, M., R. A. Blaheta, D. Henrich, J. Cinatl, B. H. Markus, H. W. Doerr, and J. Cinatl. 1996. Immunomodulatory properties of the metal chelators desferrioxamine and diethylenetriamine penta-acetic acid in vitro. Transplantation. 62:1371-4. 
451. Scholz, M., J.-U. Vogel, R. A. Blaheta, H. W. Doerr, and J. J. Cinatl. 1998. Cytomegalovirus, oxidative stress and inflammation as independent pathomechanisms: need for novel therapeutic stratagies ?, p. 90-105. In M. Scholz and H. F. Rabenau and H. W. Doerr and J. J. Cinatl (ed.), CMV-related immunopathology, vol. 21. Karger, Basel.

452. Schooley, R. T. 1990. Cytomegalovirus in the setting of infection with human immunodeficiency virus. Rev Infect Dis. 12 Suppl 7:S811-9.

453. Schoppel, K., C. Schmidt, H. Einsele, H. Hebart, and M. Mach. 1998. Kinetics of the antibody response against human cytomegalovirus- specific proteins in allogeneic bone marrow transplant recipients. J Infect Dis. 178:1233-1243.

454. Schrier, R. D., J. A. Nelson, and M. B. Oldstone. 1985. Detection of human cytomegalovirus in peripheral blood lymphocytes in a natural infection. Science. 230:104851.

455. Sedmak, D. D., D. A. Knight, N. C. Vook, and J. W. Waldman. 1994. Divergent patterns of ELAM-1, ICAM-1, and VCAM-1 expression on cytomegalovirus-infected endothelial cells. Transplantation. 58:1379-85.

456. Sedmak, D. D., W. H. Roberts, R. E. Stephens, W. J. Buesching, L. A. Morgan, D. H. Davis, and W. J. Waldman. 1990. Inability of cytomegalovirus infection of cultured endothelial cells to induce HLA class II antigen expression. Transplantation. 49:458-62.

457. Sepkowitz, K. A. 1998. Effect of HAART on natural history of AIDS-related opportunistic disorders. Lancet. 351:228-30.

458. Shanley, J. D. 1990. In vivo administration of monoclonal antibody to the NK 1.1 antigen of natural killer cells: effect on acute murine cytomegalovirus infection. J Med Virol. 30:58-60.

459. Shanley, J. D., M. C. Jordan, and J. G. Stevens. 1981. Modification by adoptive humoral immunity of murine cytomegalovirus infection. J Infect Dis. 143:231-237.

460. Shen, C. Y., W. W. Chang, S. F. Chang, M. F. Chao, E. S. Huang, and C. W. Wu. 1992. Seroepidemiology of cytomegalovirus infection among children between the ages of 4 and 12 years in Taiwan. J Med Virol. 37:72-5.

461. Sindre, H., G. E. Tjoonnfjord, H. Rollag, T. Ranneberg-Nilsen, O. P. Veiby, S. Beck, M. Degre, and K. Hestdal. 1996. Human cytomegalovirus suppression of and latency in early hematopoietic progenitor cells. Blood. 88:4526-33.

462. Singer, D. R., T. J. Fallon, W. E. Schulenburg, G. Williams, and J. Cohen. 1985. Foscarnet for cytomegalovirus retinitis. Ann Intern Med. 103:962.

463. Singh, N., V. L. Yu, L. Mieles, M. M. Wagener, R. C. Miner, and T. Gayowski. 1994. High-dose acyclovir compared with short-course preemptive ganciclovir therapy to prevent cytomegalovirus disease in liver transplant recipients. A randomized trial. Ann Intern Med. 120:375-81. 
464. Sinzger, C., K. Schmidt, J. Knapp, M. Kahl, R. Beck, J. Waldman, H. Hebart, H. Einsele, and G. Jahn. 1999. Modification of human cytomegalovirus tropism through propagation in vitro is associated with changes in the viral genome. J Gen Virol. 80:286777.

465. Slobbe-van Drunen, M. E., R. C. Vossen, F. M. Couwenberg, M. M. Hulsbosch, J. W. Heemskerk, M. C. van Dam-Mieras, and C. A. Bruggeman. 1997. Activation of protein kinase $\mathrm{C}$ enhances the infection of endothelial cells by human cytomegalovirus. Virus Res. 48:207-13.

466. Slobedman, B., and E. S. Mocarski. 1999. Quantitative analysis of latent human cytomegalovirus. J Virol. 73:4806-12.

467. Small, T. N., E. B. Papadopoulos, F. Boulad, P. Black, H. Castro-Malaspina, B. H. Childs, N. Collins, A. Gillio, D. George, A. Jakubowski, G. Heller, M. Fazzari, N. Kernan, S. MacKinnon, P. Szabolcs, J. W. Young, and O. R. RJ. 1999. Comparison of immune reconstitution after unrelated and related T-cell- depleted bone marrow transplantation: effect of patient age and donor leukocyte infusions. Blood. 93:467-80.

468. Smith, I. L., J. M. Cherrington, R. E. Jiles, M. D. Fuller, W. R. Freeman, and S. A. Spector. 1997. High-level resistance of cytomegalovirus to ganciclovir is associated with alterations in both the UL97 and DNA polymerase genes. J Infect Dis. 176:69-77.

469. Smith, J. C., I. J. Miller, Jr., R. F. Krimm, M. S. Nejad, and L. M. Beidler. 1988. A comparison of the effects of bilateral sections of the chorda tympani nerve and extirpation of the submaxillary and sublingual salivary glands on the eating and drinking patterns of the rat. Physiol Behav. 44:435-444.

470. Smith, K. L., J. K. Kulski, T. Cobain, and R. A. Dunstan. 1993. Detection of cytomegalovirus in blood donors by the polymerase chain reaction. Transfusion. 33:497503.

471. Smith, N. A., E. J. Leen, N. W. Derias, F. Nicholson, and J. S. Bingham. 1997. Massive salivary gland swelling due to primary cytomegalovirus infection in an AIDS patient. Int J STD AIDS. 8:528-9.

472. Smith, P. D., S. S. Saini, M. Raffeld, J. F. Manischewitz, and S. M. Wahl. 1992. Cytomegalovirus induction of tumor necrosis factor-alpha by human monocytes and mucosal macrophages. J Clin Invest. 90:1642-8.

473. Smyth, M. J., J. M. Kelly, A. G. Baxter, H. Korner, and J. D. Sedgwick. 1998. An essential role for tumor necrosis factor in natural killer cell-mediated tumor rejection in the peritoneum. J Exp Med. 188:1611-9.

474. Snider, W. D., D. M. Simpson, S. Nielsen, J. W. Gold, C. E. Metroka, and J. B. Posner. 1983. Neurological complications of acquired immune deficiency syndrome: analysis of 50 patients. Ann Neurol. 14:403-18. 
475. Snoeck, R., G. Andrei, and E. De Clercq. 1996. Patterns of resistance and sensitivity to antiviral compounds of drug-resistant strains of human cytomegalovirus selected in vitro. Eur J Clin Microbiol Infect Dis. 15:574-9.

476. Soderberg, C., T. D. Giugni, J. A. Zaia, S. Larsson, J. M. Wahlberg, and E. Moller. 1993. CD13 (human aminopeptidase N) mediates human cytomegalovirus infection. J Virol. 67:6576-85.

477. Soderberg-Naucler, C., K. N. Fish, and J. A. Nelson. 1997. Interferon-gamma and tumor necrosis factor-alpha specifically induce formation of cytomegalovirus-permissive monocyte-derived macrophages that are refractory to the antiviral activity of these cytokines. J Clin Invest. 100:3154-63.

478. Soderberg-Naucler, C., K. N. Fish, and J. A. Nelson. 1997. Reactivation of latent human cytomegalovirus by allogeneic stimulation of blood cells from healthy donors. Cell. 91:119-26.

479. Sohn, Y. M., M. K. Oh, K. B. Balcarek, G. A. Cloud, and R. F. Pass. 1991. Cytomegalovirus infection in sexually active adolescents. J Infect Dis. 163:460-3.

480. Solez, K., R. A. Axelsen, H. Benediktsson, J. F. Burdick, A. H. Cohen, R. B. Colvin, B. P. Croker, D. Droz, M. S. Dunnill, P. F. Halloran, and et al. 1993. International standardization of criteria for the histologic diagnosis of renal allograft rejection: the Banff working classification of kidney transplant pathology. Kidney Int. 44:411-22.

481. Solez, K., L. C. Racusen, F. Abdulkareem, E. Kemeny, E. von Willebrand, and L. D. Truong. 1997. Adhesion molecules and rejection of renal allografts. Kidney Int. $51: 1476-80$.

482. Song, M. K., M. P. Karavellas, J. C. MacDonald, D. J. Plummer, and W. R. Freeman. 2000. Characterization of reactivation of cytomegalovirus retinitis in patients healed after treatment with highly active antiretroviral therapy.LA - Eng. Retina. 20:151-5.

483. Soots, A., I. Lautenschlager, L. Krogerus, O. Saarinen, and J. Ahonen. 1998. An experimental model of chronic renal allograft rejection in the rat using triple drug immunosuppression. Transplantation. 65:42-6.

484. Sorlie, P. D., E. Adam, S. L. Melnick, A. Folsom, T. Skelton, L. E. Chambless, R. Barnes, and J. L. Melnick. 1994. Cytomegalovirus/herpesvirus and carotid atherosclerosis: the ARIC Study. J Med Virol. 42:33-7.

485. Sorlie, P. D., F. J. Nieto, E. Adam, A. R. Folsom, E. Shahar, and M. Massing. 2000. A prospective study of cytomegalovirus, herpes simplex virus 1 , and coronary heart disease: the atherosclerosis risk in communities (ARIC) study. Arch Intern Med. 160:202732.

486. Span, A. H., P. M. Frederik, G. Grauls, G. P. Van Boven, and C. A. Bruggeman. 1993. CMV induced vascular injury: an electron-microscopic study in the rat. In Vivo. 7:567-73. 
487. Span, A. H., G. Grauls, F. Bosman, C. P. van Boven, and C. A. Bruggeman. 1992. Cytomegalovirus infection induces vascular injury in the rat. Atherosclerosis. 93:41-52.

488. Spector, S. A. 1999. Oral ganciclovir. Adv Exp Med Biol. 458:121-7.

489. Spector, T., J. A. Harrington, and D. J. Porter. 1991. Herpes and human ribonucleotide reductases. Inhibition by 2-acetylpyridine 5-[(2-chloroanilino)-thiocarbonyl]thiocarbonohydrazone (348U87). Biochem Pharmacol. 42:91-6.

490. Speir, E., E. S. Huang, R. Modali, M. B. Leon, F. Shawl, T. Finkel, and S. E. Epstein. 1995. Interaction of human cytomegalovirus with p53: possible role in coronary restenosis. Scand J Infect Dis Suppl. 99:78-81.

491. Speir, E., T. Shibutani, Z. X. Yu, V. Ferrans, and S. E. Epstein. 1996. Role of reactive oxygen intermediates in cytomegalovirus gene expression and in the response of human smooth muscle cells to viral infection. Circ Res. 79:1143-52.

492. Speiser, D. E., J. M. Tiercy, N. Rufer, C. Grundschober, A. Gratwohl, B. Chapuis, C. Helg, C. C. Loliger, M. K. Siren, E. Roosnek, and M. Jeannet. 1996. High resolution HLA matching associated with decreased mortality after unrelated bone marrow transplantation. Blood. 87:4455-62.

493. Springer, T. A. 1990. Adhesion receptors of the immune system. Nature. 346:42534.

494. Springer, T. A. 1995. Traffic signals on endothelium for lymphocyte recirculation and leukocyte emigration. Annu Rev Physiol. 57:827-72.

495. Stagno, S., R. F. Pass, G. Cloud, W. J. Britt, R. E. Henderson, P. D. Walton, D. A. Veren, F. Page, and C. A. Alford. 1986. Primary cytomegalovirus infection in pregnancy. Incidence, transmission to fetus, and clinical outcome. Jama. 256:1904-8.

496. Stagno, S., R. F. Pass, M. E. Dworsky, R. E. Henderson, E. G. Moore, P. D. Walton, and C. A. Alford. 1982. Congenital cytomegalovirus infection: The relative importance of primary and recurrent maternal infection. N Engl J Med. 306:945-9.

497. Stals, F. S., Bosman, F. Van-Boven, C.P., Bruggeman, C.A. 1990. An animal model for therapeutic intervention studies of $\mathrm{CMV}$ infection in the immunocompromised host. Arch. Virol. 114:91-107.

498. Stals, F. S., E. de Clercq, and C. A. Bruggeman. 1991. Comparative activity of (S)1-(3-hydroxy-2-phosphonylmethoxypropyl)cytosine and 9-(1,3-dihydroxy-2propoxymethyl)guanine against rat cytomegalovirus infection in vitro and in vivo. Antimicrob Agents Chemother. 35:2262-6.

499. Stals, F. S., S. S. Wagenaar, J. S. Kloover, W. Y. Vanagt, and C. A. Bruggeman. 1996. Combinations of ganciclovir and antibody for experimental CMV infections. Antiviral Res. 29:61-4. 
500. Stals, F. S., A. Zeytinoglu, M. Havenith, E. de Clercq, and C. A. Bruggeman. 1993. Rat cytomegalovirus-induced pneumonitis after allogeneic bone marrow transplantation: effective treatment with (S)-1-(3-hydroxy-2-phosphonyl-methoxypropyl)cytosine. Antimicrob Agents Chemother. 37:218-23.

501. Stanier, P., A. D. Kitchen, D. L. Taylor, and A. S. Tyms. 1992. Detection of human cytomegalovirus in peripheral mononuclear cells and urine samples using PCR. Mol Cell Probes. 6:51-8.

502. Stary, H. C. 1989. Evolution and progression of atherosclerotic lesions in coronary arteries of children and young adults. Arteriosclerosis. 9:I19-32.

503. Steffens, H. P., S. Kurz, R. Holtappels, and M. J. Reddehase. 1998. Preemptive CD8 T-cell immunotherapy of acute cytomegalovirus infection prevents lethal disease, limits the burden of latent viral genomes, and reduces the risk of virus recurrence. J Virol. 72:1797-804.

504. Stein, J., H. D. Volk, C. Liebenthal, D. H. Kruger, and S. Prosch. 1993. Tumour necrosis factor alpha stimulates the activity of the human cytomegalovirus major immediate early enhancer/promoter in immature monocytic cells. J Gen Virol. 74:2333-8.

505. Steinhoff, G., M. Behrend, and A. Haverich. 1991. Signs of endothelial inflammation in human heart allografts. Eur Heart J. 12 Suppl D:141-3.

506. Steinhoff, G., X. M. You, C. Steinmuller, D. Bauer, M. L. Lohmann-Matthes, C. A. Bruggeman, and A. Haverich. 1996. Enhancement of cytomegalovirus infection and acute rejection after allogeneic lung transplantation in the rat. Transplantation. 61:1250-60.

507. Stocchi, R., K. N. Ward, R. Fanin, M. Baccarani, and J. F. Apperley. 1999. Management of human cytomegalovirus infection and disease after allogeneic bone marrow transplantation. Haematologica. 84:71-9.

508. Stone, T. W., and G. J. Jaffe. 2000. Reversible bull's-eye maculopathy associated with intravitreal fomivirsen therapy for cytomegalovirus retinitis. Am J Ophthalmol. 130:242-3.

509. Streblow, D. N., C. Soderberg-Naucler, J. Vieira, P. Smith, E. Wakabayashi, F. Ruchti, K. Mattison, Y. Altschuler, and J. A. Nelson. 1999. The human cytomegalovirus chemokine receptor US28 mediates vascular smooth muscle cell migration. Cell. 99:51120.

510. Sullivan, V., K. K. Biron, C. Talarico, S. C. Stanat, M. Davis, L. M. Pozzi, and D. M. Coen. 1993. A point mutation in the human cytomegalovirus DNA polymerase gene confers resistance to ganciclovir and phosphonylmethoxyalkyl derivatives. Antimicrob Agents Chemother. 37:19-25.

511. Sullivan, V., C. L. Talarico, S. C. Stanat, M. Davis, D. M. Coen, and K. K. Biron. 1992. A protein kinase homolog controls phosphorylation of ganciclovir in human cytomegalovirus-infected cells. Nature. 358:162-4. 
512. Takenaka, K., H. Gondo, K. Tanimoto, K. Nagafuji, T. Fujisaki, S. Mizuno, T. Miyamoto, T. Okamura, S. Hayashi, T. Eto, K. Osaki, K. Yamasaki, T. Shibuya, N. Harada, T. Teshima, E. Matsuishi, T. Minematsu, Y. Minamishima, M. Harada, and Y. Niho. 1997. Increased incidence of cytomegalovirus (CMV) infection and CMV- associated disease after allogeneic bone marrow transplantation from unrelated donors. The Fukuoka Bone Marrow Transplantation Group. Bone Marrow Transplant. 19:241-8.

513. Taylor, P. M., M. L. Rose, M. H. Yacoub, and R. Pigott. 1992. Induction of vascular adhesion molecules during rejection of human cardiac allografts. Transplantation. 54:4517.

514. Taylor-Wiedeman, J., J. G. Sissons, L. K. Borysiewicz, and J. H. Sinclair. 1991. Monocytes are a major site of persistence of human cytomegalovirus in peripheral blood mononuclear cells. J Gen Virol. 72:2059-64.

515. Taylor-Wiedeman, J., P. Sissons, and J. Sinclair. 1994. Induction of endogenous human cytomegalovirus gene expression after differentiation of monocytes from healthy carriers. J Virol. 68:1597-604.

516. Tilney, N. L., W. D. Whitley, J. R. Diamond, J. W. Kupiec-Weglinski, and D. H. Adams. 1991. Chronic rejection--an undefined conundrum. Transplantation. 52:389-98.

517. Tomasec, P., V. M. Braud, C. Rickards, M. B. Powell, B. P. McSharry, S. Gadola, V. Cerundolo, L. K. Borysiewicz, A. J. McMichael, and G. W. Wilkinson. 2000. Surface expression of HLA-E, an inhibitor of natural killer cells, enhanced by human cytomegalovirus gpUL40. Science. 287:1031.

518. Tookey, P. A., A. E. Ades, and C. S. Peckham. 1992. Cytomegalovirus prevalence in pregnant women: the influence of parity. Arch Dis Child. 67:779-83.

519. Topilko, A., and S. Michelson. 1994. Morphological and cytochemical analysis of human cytomegalovirus inoculum: correlation of free particles in inoculum with counterparts in infected cells. Res Virol. 145:65-73.

520. Torok-Storb, B., M. Boeckh, C. Hoy, W. Leisenring, D. Myerson, and T. Gooley. 1997. Association of specific cytomegalovirus genotypes with death from myelosuppression after marrow transplantation. Blood. 90:2097-102.

521. Tumilowicz, J. J. 1990. Characteristics of human arterial smooth muscle cell cultures infected with cytomegalovirus. In Vitro Cell Dev Biol. 26:1144-50.

522. Tural, C., J. Romeu, G. Sirera, D. Andreu, M. Conejero, S. Ruiz, A. Jou, A. Bonjoch, L. Ruiz, A. Arno, and B. Clotet. 1998. Long-lasting remission of cytomegalovirus retinitis without maintenance therapy in human immunodeficiency virus-infected patients. $J$ Infect Dis. 177:1080-3.

523. Ulbrecht, M., S. Martinozzi, M. Grzeschik, H. Hengel, J. W. Ellwart, M. Pla, and E. H. Weiss. 2000. Cutting edge: the human cytomegalovirus UL40 gene product contains a ligand for HLA-E and prevents NK cell-mediated lysis. J Immunol. 164:5019-22. 
524. Ustinov, J. A., R. J. Loginov, P. M. Mattila, V. K. Nieminen, J. I. Suni, P. J. Hayry, and I. T. Lautenschlager. 1991. Cytomegalovirus infection of human kidney cells in vitro. Kidney Int. 40:954-60.

525. van Dam, J. G., J. G. Damoiseaux, H. A. Van der Heijden, G. Grauls, P. J. Van Breda Vriesman, and C. A. Bruggeman. 1997. Infection with rat cytomegalovirus (CMV) in the immunocompromised host is associated with the appearance of a $\mathrm{T}$ cell population with reduced CD8 and T cell receptor (TCR) expression. Clin Exp Immunol. 110:349-57.

526. van Dam, J. G., F. Li, M. Yin, X. M. You, G. Grauls, G. Steinhoff, and C. A. Bruggeman. 2000. Effects of cytomegalovirus infection and prolonged cold ischemia on chronic rejection of rat renal allografts. Transpl Int. 13:54-63.

527. van Dorp, W. T., E. Jonges, C. A. Bruggeman, M. R. Daha, L. A. van Es, and F. J. van Der Woude. 1989. Direct induction of MHC class I, but not class II, expression on endothelial cells by cytomegalovirus infection. Transplantation. 48:469-72.

528. van Dorp, W. T., P. A. van Wieringen, E. Marselis-Jonges, C. A. Bruggeman, M. R. Daha, L. A. van Es, and F. van der Woude. 1993. Cytomegalovirus directly enhances MHC class I and intercellular adhesion molecule-1 expression on cultured proximal tubular epithelial cells. Transplantation. 55:1367-71.

529. van Son, W. J., E. F. de Maar, W. van Der Bij, A. P. van Den Berg, E. A. Verschuuren, and T. H. The. 1999. Overcoming the problem of cytomegalovirus infection after organ transplantation: calling for Heracles? Intervirology. 42:285-90.

530. Ventura, H. O., M. R. Mehra, F. W. Smart, and D. D. Stapleton. 1995. Cardiac allograft vasculopathy: current concepts. Am Heart J. 129:791-9.

531. Vink, C., E. Beuken, and C. A. Bruggeman. 2000. Complete DNA sequence of the rat cytomegalovirus genome. J Virol. 74:7656-65.

532. Visseren, F. L., K. P. Bouter, M. J. Pon, J. B. Hoekstra, D. W. Erkelens, and R. J. Diepersloot. 1997. Patients with diabetes mellitus and atherosclerosis; a role for cytomegalovirus? Diabetes Res Clin Pract. 36:49-55.

533. von Willebrand, E., V. Jurcic, H. Isoniemi, P. Hayry, T. Paavonen, and L. Krogerus. 1997. Adhesion molecules and their ligands in chronic rejection of human renal allografts. Transplant Proc. 29:1530-1.

534. von Willebrand, E., I. Lautenschlager, L. Krogerus, P. Hayry, H. Isoniemi, and K. Salmela. 1996. Adhesion molecules and activation markers in acute rejection of human renal allografts. Transpl Immunol. 4:57-8.

535. von Willebrand, E., E. Pettersson, J. Ahonen, and P. Hayry. 1986. CMV infection, class II antigen expression, and human kidney allograft rejection. Transplantation. 42:3647. 
536. Vossen, R. C., J. G. Derhaag, M. E. Slobbe van Drunen, A. M. Duijvestijn, M. C. van Dam Mieras, and C. A. Bruggeman. 1996. A dual role for endothelial cells in cytomegalovirus infection? A study of cytomegalovirus infection in a series of rat endothelial cell lines. Virus Res. 46:65-74.

537. Vossen, R. C., M. C. Persoons, M. E. Slobbe van Drunen, C. A. Bruggeman, and M. C. van Dam Mieras. 1997. Intracellular thiol redox status affects rat cytomegalovirus infection of vascular cells. Virus Res. 48:173-83.

538. Vrabec, T. R., V. F. Baldassano, and S. M. Whitcup. 1998. Discontinuation of maintenance therapy in patients with quiescent cytomegalovirus retinitis and elevated CD4+ counts. Ophthalmology. 105:1259-64.

539. Wachsman, M., B. G. Petty, K. C. Cundy, H. S. Jaffe, P. E. Fisher, A. Pastelak, and P. S. Lietman. 1996. Pharmacokinetics, safety and bioavailability of HPMPC (cidofovir) in human immunodeficiency virus-infected subjects. Antiviral Res. 29:153-61.

540. Wagner, R. P., H. Tian, M. J. McPherson, P. S. Latham, and J. M. Orenstein. 1996. AIDS-associated infections in salivary glands: autopsy survey of 60 cases. Clin Infect Dis. 22:369-71.

541. Waldman, W. J., and D. A. Knight. 1996. Cytokine-mediated induction of endothelial adhesion molecule and histocompatibility leukocyte antigen expression by cytomegalovirus-activated T cells. Am J Pathol. 148:105-19.

542. Walmsley, S. L., E. Chew, S. E. Read, H. Vellend, I. Salit, A. Rachlis, and M. M. Fanning. 1988. Treatment of cytomegalovirus retinitis with trisodium phosphonoformate hexahydrate (Foscarnet). J Infect Dis. 157:569-72.

543. Waxman, A. B., S. J. Goldie, H. Brett Smith, and R. A. Matthay. 1997. Cytomegalovirus as a primary pulmonary pathogen in AIDS. Chest. 111:128-34.

544. Welsh, R. M., J. O. Brubaker, M. Vargas-Cortes, and C. L. O'Donnell. 1991. Natural Killer (NK) cell response to virus infections in mice with severe combined immunodeficiency. The stimulation of NK cells and the NK cell-dependent control of virus infections occur independently of B and T cell function. J. Exp. Med. 173:1053-1063.

545. Wiertz, E. J., T. R. Jones, L. Sun, M. Bogyo, H. J. Geuze, and H. L. Ploegh. 1996. The human cytomegalovirus US11 gene product dislocates MHC class I heavy chains from the endoplasmic reticulum to the cytosol. Cell. 84:769-79.

546. Wiertz, E. J. H. J., S. Mukherjee, and H. L. Ploegh. 1997. Viruses use stealth technology to escape from the host immune system. Molecular medicine today. march:116-123.

547. Wilkes, M. S., A. H. Fortin, J. C. Felix, T. A. Godwin, and W. G. Thompson. 1988. Value of necropsy in acquired immunodeficiency syndrome. Lancet. 2:85-8. 
548. Wingard, J. R., D. Y. Chen, W. H. Burns, D. J. Fuller, H. G. Braine, A. M. Yeager, H. Kaiser, P. J. Burke, M. L. Graham, G. W. Santos, and et al. 1988. Cytomegalovirus infection after autologous bone marrow transplantation with comparison to infection after allogeneic bone marrow transplantation. Blood. 71:1432-7.

549. Winston, D. J., and R. P. Gale. 1991. Prevention and treatment of cytomegalovirus infection and disease after bone marrow transplantation in the 1990s. Bone Marrow Transplant. 8:7-11.

550. Winston, D. J., W. G. Ho, and R. E. Champlin. 1990. Cytomegalovirus infections after allogeneic bone marrow transplantation. Rev Infect Dis. 12:S776-92.

551. Wolf, D. G., D. J. Lee, and S. A. Spector. 1995. Detection of human cytomegalovirus mutations associated with ganciclovir resistance in cerebrospinal fluid of AIDS patients with central nervous system disease. Antimicrob Agents Chemother. 39:2552-4.

552. Wolf, D. G., I. Yaniv, A. Honigman, I. Kassis, T. Schonfeld, and S. Ashkenazi. 1998. Early emergence of ganciclovir-resistant human cytomegalovirus strains in children with primary combined immunodeficiency. J Infect Dis. 178:535-8.

553. Wong, T., S. Lavaud, O. Toupance, J. Carquin, A. Wynckel, J. P. Melin, C. Hanrotel, and J. Chanard. 1993. Failure of acyclovir to prevent cytomegalovirus infection in renal allograft recipients. Transpl Int. 6:285-9.

554. Wright, J. F., A. Kurosky, and S. Wasi. 1994. An endothelial cell-surface form of annexin II binds human cytomegalovirus. Biochem Biophys Res Commun. 198:983-9.

555. Wu, G. S., J. Walker, and N. A. Rao. 1993. Effect of deferoxamine on retinal lipid peroxidation in experimental uveitis. Invest Ophthalmol Vis Sci. 34:3084-9.

556. Yang, H., and R. Datema. 1991. Prolonged and potent therapeutic and prophylactic effects of (S)-1-[(3-hydroxy-2-phosphonylmethoxy)propyl]cytosine against herpes simplex virus type 2 infections in mice. Antimicrob Agents Chemother. 35:1596600 .

557. Yilmaz, S., P. K. Koskinen, E. Kallio, C. A. Bruggeman, P. J. Hayry, and K. B. Lemstrom. 1996. Cytomegalovirus infection-enhanced chronic kidney allograft rejection is linked with intercellular adhesion molecule-1 expression. Kidney Int. 50:526-37.

558. Zhou, L., T. C. Harder, U. Ullmann, and P. Rautenberg. 1999. Rapid detection by reverse hybridization of mutations in the UL97 gene of human cytomegalovirus conferring resistance to ganciclovir. J Clin Virol. 13:53-9.

559. Zhou, Y. F., M. B. Leon, M. A. Waclawiw, J. J. Popma, Z. X. Yu, T. Finkel, and S. E. Epstein. 1996. Association between prior cytomegalovirus infection and the risk of restenosis after coronary atherectomy. N Engl J Med. 335:624-30. 
560. Zhou, Y. F., M. Shou, E. Guetta, R. Guzman, E. F. Unger, Z. X. Yu, J. Zhang, T. Finkel, and S. E. Epstein. 1999. Cytomegalovirus infection of rats increases the neointimal response to vascular injury without consistent evidence of direct infection of the vascular wall. Circulation. 100:1569-75.

561. Zhou, Y. F., M. Shou, R. F. Harrell, Z. X. Yu, E. F. Unger, and S. E. Epstein. 2000. Chronic non-vascular cytomegalovirus infection: effects on the neointimal response to experimental vascular injury. Cardiovasc Res. 45:1019-25.

562. Zhu, H., Y. Shen, and T. Shenk. 1995. Human cytomegalovirus IE1 and IE2 proteins block apoptosis. J Virol. 69:7960-70.

563. Zhu, J., A. A. Quyyumi, J. E. Norman, G. Csako, and S. E. Epstein. 1999. Cytomegalovirus in the pathogenesis of atherosclerosis: the role of inflammation as reflected by elevated C-reactive protein levels. J Am Coll Cardiol. 34:1738-43.

564. Zhu, J., A. A. Quyyumi, J. E. Norman, G. Csako, M. A. Waclawiw, G. M. Shearer, and S. E. Epstein. 2000. Effects of total pathogen burden on coronary artery disease risk and C-reactive protein levels. Am J Cardiol. 85:140-6.

565. Ziegler, H., R. Thale, P. Lucin, W. Muranyi, T. Flohr, H. Hengel, H. Farrell, W. Rawlinson, and U. H. Koszinowski. 1997. A mouse cytomegalovirus glycoprotein retains MHC class I complexes in the ERGIC/cis-Golgi compartments. Immunity. 6:57-66. 
